This document is downloaded from DR-NTU (https://dr.ntu.edu.sg) Nanyang Technological University, Singapore.

An in-vitro evaluation of C-di-GMP mediated biofilm dispersal as a potential strategy to restore antimicrobial efficacy

Roizman, Dan

2018

https://hdl.handle.net/10356/87655

https://doi.org/10.32657/10220/46811 



AN IN-VITRO EVALUATION OF C-DI-GMP MEDIATED BIOFILM DISPERSAL AS A POTENTIAL STRATEGY TO RESTORE ANTIMICROBIAL EFFICACY

\section{DAN ROIZMAN}

Interdisciplinary Graduate School

Singapore Centre for

Environmental Life Sciences Engineering

2018 


\title{
AN IN-VITRO EVALUATION OF C-DI-GMP MEDIATED BIOFILM DISPERSAL AS A POTENTIAL STRATEGY TO RESTORE ANTIMICROBIAL EFFICACY
}

Dan Roizman

\author{
Interdisciplinary Graduate School \\ Singapore Centre for \\ Environmental Life Sciences Engineering
}

A thesis submitted to the Nanyang Technological University in partial fulfillment of the requirement for the degree of Doctor of Philosophy 


\section{Acknowledgments}

My time spent in graduate school was a memorable undertaking, full of adventure, excitement, ups and downs, and mainly, a tremendous learning experience. In that sense, first and foremost, I would like to thank my supervisor, Assoc. Prof. Yang Liang, for showing me the path to do great science, for his guidance and his encouragement. I would like to thank my mentor, Assoc. Prof. Scott Rice, for his important advice. I would also like to thank my co-supervisor Assoc. Prof. Tan Choon Hong for his assistance along the way.

I am especially grateful to Dr. Céline Vidaillac. Words cannot express my sincere, heartfelt gratitude for all of her efforts to assist me in my scientific endeavor and my career. I would like to thank her for her guidance and inspiration, for being a beacon in stormy seas.

I thank the director of SCELSE, Prof. Staffan Kjelleberg, for his vision, managing a unique and unusual interdisciplinary center, and for the opportunity to take part in cutting-edge science. Thanks to SCELSE graduate programme director, Assoc. Prof. Sanjay Swarup, for his continued support and confidence in me. I also thank my former supervisor, Prof. Yehuda Cohen, for providing me the opportunity to partake in this research experience. Thanks to all the people at IGS for supporting my research and me.

I would like to thank all of Prof. Yang's group members, my fellow Ph.D. students and predecessors. A special thank you to Chin Joyce and Seng Zi Jing, for their continued assistance to my research. Thanks to my dear friends, 
colleagues and former colleagues in SCELSE: To Dr. Damien Keogh, also for taking the time and proofreading my thesis. Asst. Prof. Enrico Marsili, Dr. Thomas Seviour, Dr. Jamie Hinks, Dr. Sujatha Subramoni, Dr. Lucinda Doyle, Dr. Carlo Santoro, Dr. Nguyen Mai Phuong, Dr. Lim Chu Ping, Dr. Gurjeet Singh Kohli, Dr. Arthur Matysik and Dr. Tay Martin for the joint research; Dr. Prasanna Jogdeo, Samarpita Roy, Dr. Kumaravel Kandaswamy, Han Evelina, Dr. Sumitra Mitra and Dr. Su Chew. Thank you all for being there for me. To all SCELSE laboratory, research, administration staffs, especially Priscilla Lefort, Loh Ying Ting, Natasha Oberi-Lee and Chew Ley Byan, thanks for joining me and assisting on this ride called Ph.D. It's lovely to have good friends along the way.

Last but not least, I would like to thank my family for their love, support, and reinforcement. First, for my wonderful wife, Kathrin Roizman, that her marvelous figures and drawings accompany this thesis. Thanks for being the support, for your endless love and attention, for being just who you are, the woman I love and admire. This Singapore adventure was all there to find each other. I thank my parents Genny and Yefim Roizman for encouraging me all my life to pursue my dreams and aspirations. My three sisters: Michal, Rinat \& Anat Roizman, for being the best sisters in the world, for their help and admiration. And of course, my grandmother Dr. Aviva Shimkin, for being the best role model a grandson can ask for. Thank you, my dear family, I love you all. I succeeded in this Ph.D. because of you. 


\section{Summary}

The World Health Organization (WHO) has deemed antimicrobial resistance (AMR) in bacteria to be a critical threat to society (World Health Organization 2016, World Health Organization 2017). AMR and specifically multi-drug resistant (MDR) bacteria represent an increasingly serious public health concern worldwide. The need for novel therapeutic approaches to prevent and/or eradicate microbial infections is urgent. AMR can evolve due to mutations that reduce antibiotic susceptibility or due to activation of various innate mechanisms of tolerance, for example, biofilm formation. Bacteria exhibit a complex life-cycle, whereby they can switch from a planktonic, freeliving form, to a densely packed biofilm form. Biofilms are an aggregated, sessile, often surface attached form of bacterial mode of growth. Biofilms provide complex $3 \mathrm{D}$ structures to bacterial communities, allowing them to interact with each other and respond to changes in their environment differently than their planktonic counterparts (Costerton, Geesey et al. 1978). $60-80 \%$ of infections are biofilm-related, once biofilms attach and mature bacteria can exhibit 10 to 1000 times less susceptibility to antimicrobials (Høiby, Bjarnsholt et al. 2010).

The use of dispersal agents has been suggested as a potential treatment strategy against microbial biofilms (Banin, Brady et al. 2006, Frederiksen, Pressler et al. 2006, Landini, Antoniani et al. 2010, Barraud, J. Kelso et al. 2015). This strategy consists of manipulating biofilm dispersal to induce changes in biofilm matrix and metabolic states of biofilm cells. These changes are believed to enhance antimicrobial efficacy, increasing antimicrobial killing 
efficiency, shortening duration of treatment and reducing the risk of emergence of resistance. Investigating the effectiveness of biofilm dispersal as part of an antimicrobial treatment strategy is challenging, and the consequences and the underlying mechanisms of such strategy remain unclear. So far, no dispersal agents have yet been used extensively in a clinical setting (Fleming and Rumbaugh 2017). The present research aims at providing knowledge about how c-di-GMP mediated biofilm dispersal will impact antimicrobial synergy treatment against bacterial biofilms and whether it will result in the emergence of resistant/tolerant variant populations.

To achieve these objectives, a strain of Pseudomonas aeruginosa, PAO1/p $\mathrm{p}_{B A D^{-}} y$ hjH which carries an inducible phosphodiesterase (PDE) enzyme allowing for controlled biofilm dispersal, was utilized (Chua, Tan et al. 2013). A strain PAO1/pJN105 carrying an empty vector plasmid was used as a control. Using antimicrobial agents, alone or in combination, under static in-vitro conditions, c-di-GMP mediated biofilm dispersal was shown to enhance drug synergy against $P$. aeruginosa biofilms (Chapter 2), without increasing the risk of releasing resistant/tolerant variants (Chapter 3 ). The potential of biofilm dispersal strategy was also assessed against an evolved AMR strain (Chapter 4). A colistin-resistant derivative of $P A O 1 / \mathrm{p}_{B A D^{-}} y$ hjH was generated and characterized: Significant differences in morphology, motility, and virulence factor production were identified between the parent $\mathrm{PAO} 1 / \mathrm{p}_{B A D^{-}} y \mathrm{hjH}$ strain and its colistin resistant derivative. Whole genome sequencing and analysis revealed point mutations in $p h o Q$ and $I p x C$, two genes involved in the biosynthesis of lipid-A located in the bacterial membranes and the biological target of colistin. These mutations resulted in upregulated expression of the 
arn operon which is known to lead to the modification of Lipid-A. The colistinresistant derivative also showed enhanced susceptibility to carbapenems and glycopeptides, suggesting that the development of colistin resistance resulted in a trade-off in sensitivity to other classes of antimicrobials. This was further explored by testing biofilms for sensitivity to a range of antimicrobial combinations. It was observed that colistin plus vancomycin demonstrated a synergistic effect only against the dispersed biofilms of the colistin resistant strain. The results of this work indicate the potential of c-di-GMP mediated biofilm dispersal as a potential strategy for efficient treatment of biofilmassociated infections. 


\section{Author's Publications}

\section{Journal Publications}

The-Thien Tran, Celine Vidaillac, Hong Yu, Valerie F. L. Yong, Dan Roizman, Ravishankar Chandrasekaran, Albert Y. H. Lim, Teck Boon Low, Gan Liang Tan, John A. Abisheganaden, Mariko Siyue Koh, Jeanette Teo, Sanjay Haresh Chotrimall, Kunn Hadinoto Ong. (2018). "A new therapeutic avenue for bronchiectasis: Dry powder inhaler of ciprofloxacin nanoplex exhibits superior ex-vivo mucus permeability and antibacterial efficacy to its native ciprofloxacin counterpart". International Journal of Pharmaceutics. 2018 Aug, 547 (1-2), 368-376.

Dan Roizman, Celine Vidaillac, Michael Givskov, Liang Yang. (2017). "In-vitro evaluation of biofilm dispersal as a therapeutic strategy to restore antimicrobial efficacy". Antimicrobial Agents \& Chemotherapy. 2017 Oct, 61(10) e01088-17.

Chun Ping Lim, Phuong Nguyen Quoc Mai, Dan Roizman Sade, Yee Cheong Lam, Yehuda Cohen. (2016). "Biofilm development of an opportunistic model bacterium analysed at high spatiotemporal resolution in the framework of a precise flow cell". npj Biofilms and Microbiomes. 2016 Oct, 2, 16023.

Kateryna Artyushkova, Dan Roizman, Carlo Santoro, Lucinda Elizabeth Doyle, Abeed Fatima Mohidin, Plamen Atanassov, Enrico Marsili. (2016). "Anodic biofilms as the interphase for electroactive bacterial growth on carbon veil". Biointerphases. 2016 Sep, 11(3) 031013-1-10. 
Thomas Seviour, Piyarat Weerachanchai, Jamie Hinks, Dan Roizman, Scott A. Rice, Linlu Bai, Jong-Min Lee, Staffan Kjelleberg. (2014). "Solvent optimization for bacterial extracellular matrices: a solution for the insoluble". RSC Advances. 2014 Dec, 5(10), 7469-7478.

Martin Tay, Dan Roizman, Yehuda Cohen, Tim Tolker-Nielsen, Michael Givskov, Yang Liang. (2014). “Draft genome sequence of the model naphthalene-utilizing organism Pseudomonas putida OUS82". Genome Announcements. 2014 Feb, 2(1) e01161-13.

\section{Poster Presentations}

Dan Roizman, Celine Vidaillac, Michael Givskov, Liang Yang (2018). "Utilization of Induced Dispersal as a Strategy to Eradicate Biofilms of Colistin Resistant Strains." Biofilms 8, Aarhus, Denmark, 27-29 May 2018.

Dan Roizman, Celine Vidaillac, Michael Givskov, Liang Yang. (2017). “In-vitro evaluation of biofilm dispersal as a therapeutic strategy to restore antimicrobial efficacy". IUMS 2017, Singapore, 17-21 July 2017.

Dan Roizman, Celine Vidaillac, Michael Givskov, Liang Yang. (2017). “In-vitro evaluation of biofilm dispersal as a therapeutic strategy to restore antimicrobial efficacy". ECCMID 2017, Vienna, Austria, 22-25 April 2017. 


\section{Table of Contents}

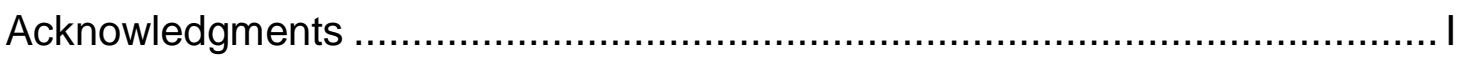

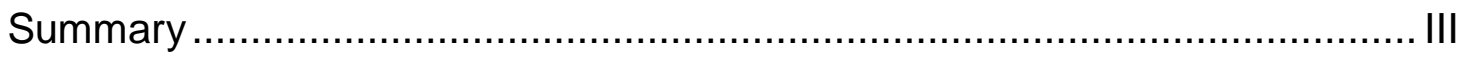

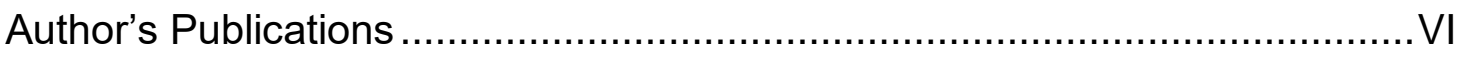

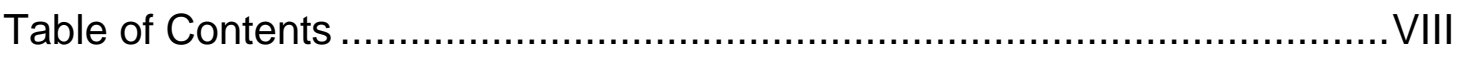

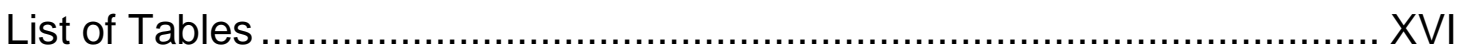

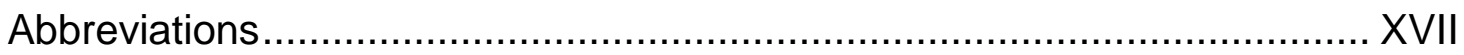

Chapter 1: Literature Review ................................................. 1 -

1.1 Antimicrobial Resistance (AMR) ….................................................... - 1 -

1.2 Antibiotic Resistance Mechanisms ............................................................. - 3 -

1.3 Biofilm-associated tolerance ................................................................... - 7 -

1.4 Microbial Life on Surfaces.................................................................. 10 -

1.5 Biofilm dispersal ................................................................................. 14 -

1.6 P. aeruginosa as a Model Gram-negative Biofilm Former .......................... - 19 -

1.7 Antibiotic testing strategies ............................................................... 22 -

1.8 Research Gaps ................................................................................ 31 -

1.9 Aims and Scope ........................................................................ 32

Chapter 2: Evaluation of c-di-GMP Mediated Biofilm Dispersal as a Strategy

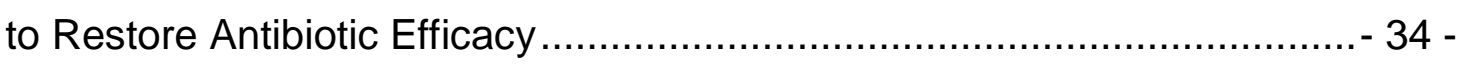





2.3 Results...................................................................................... 42 -

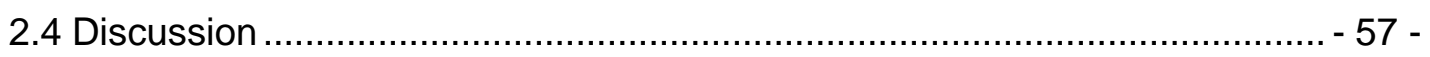


Chapter 3: Impact of c-di-GMP Mediated Biofilm Dispersal on the Rate of



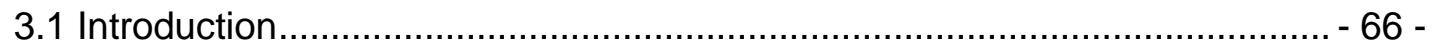

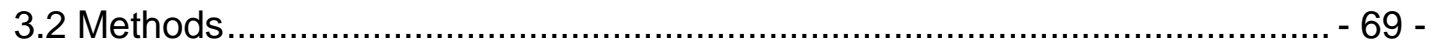

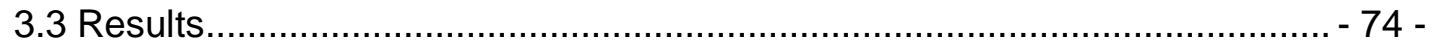

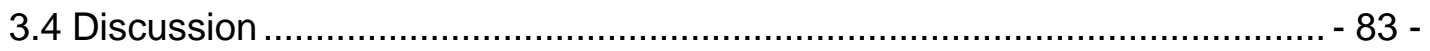

Chapter 4: Utilization of Induced c-di-GMP Mediated Dispersal as a Strategy to Eradicate Biofilms of Colistin Resistant Strains ................................... 88 -

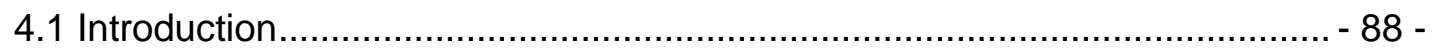

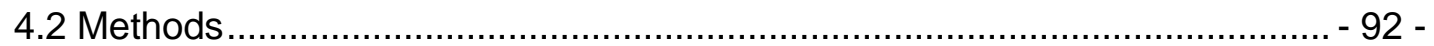

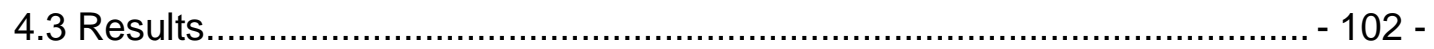

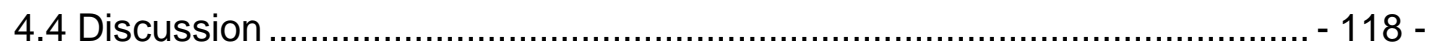

Conclusions and Future Prospects ................................................ 128 -



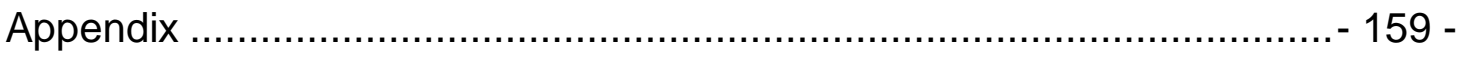




\section{List of Figures}

Figure 1.1 A schematic representing tolerance and resistance mechanisms in biofilms, antibiotic penetration (yellow gradient) into the inner levels of biofilm is slowed down due to viscosity and ionic and hydrophobic interactions with EPS constituents (eDNA, Psl). Sub-optimal antibiotic concentration leads to higher expression levels of resistance/tolerance genes in the inner biofilm levels (green); and heterogenic local environments in biofilms due to oxygen and nutrient gradients (red gradient). All of the above lead to different metabolic rates and heterogenic gene expression. Antibiotic treatment thus results in partial efficacy; not all cells are killed (red bacteria) some live cells always remain (green bacteria). Finally, the biofilm population harbors higher frequency of persister cells (purple bacteria). $9-$

Figure 1.2 A scheme representing passive and active dispersal of bacterial biofilms. Passive dispersal (Top) can be caused by external forces such as shearing while active dispersal (Bottom) is the result of different signal transduction cascades, usually involves enzymes that degrade components of the EPS matrix (Figure taken from: (Fleming and Rumbaugh 2017). $-15-$

Figure 1.3 The biofilm life-cycle under positive control by the intracellular c-di-GMP concentration. C-di-GMP is the product of DGC enzyme family and degraded by the PDE enzyme family. The image shows the correlation between high c-di-GMP (more DGC activity) and biofilm growth and low concentrations (more PDE activity) and dispersal. The P.aeruginosa construct used throughout this thesis overexpresses the PDE YhjH when induced, thus facilitating higher levels of biofilm dispersal. $-16-$

Figure 1.4 A scheme describing the mechanisms of signal transduction which culminate in changes to the intracellular c-di-GMP concentration that result in differential expression in cells and phenotypic changes (Taken from McDougald, Rice et al. 2011). 18

Figure 1.5 $P$. aeruginosa is a versatile organism which exists in many environments, including the human body. When a patient's immune system is compromised, the opportunistic $P$. aeruginosa can be found in various related infections. Images are taken from https://thenounproject.com under creative commons license. $-20-$

Figure 1.6 Examples of chronic infections predominated by $P$. aeruginosa biofilm. Scanning electron microscope (images.fineartamerica.com), contact lens-related eye infection (www.honolulugeclinic.com), infected lung tissue of cystic fibrosis patient 
(microbewiki. keynon.edu), urinary tract infection (jucing-for-health.com), neonatal and pediatric-related infection (dermaamin.com), chronic wound infection (www.medetec.co.uk), folliculitis skin related infection (callisto.ggsrv.com), nail infection (www.danderm-pdv.is.kkk.dk). $-21-$

Figure 1.7 Microtiter plate broth microdilution method combined to determine MIC, combined with REMA assay to determine MBC. A challenge plate is prepared with a gradient of antibiotic concentrations, in which bacteria are inoculated at $\sim 5 \times 10^{5}$ $\mathrm{CFU} / \mathrm{mL}$ and left overnight. MIC is the concentration that inhibits $50 \%$ of growth. The REMA assay measures MBC: Resazurin in wells which contain active cells becomes reduced (pink) and highly-fluorescent, otherwise remains blue. MBC is the concentration which results in $99 \%$ killing of bacteria. $-24-$

Figure 1.8 The use of CBD to exclusively measure susceptibility of the biofilm fraction of bacteria. The challenge plate depicted here is organized as a checkerboard assay, with criss-cross gradients of two drugs, horizontally and vertically. MBIC is measured as turbidity, and the REMA assay is utilized to measure MBEC. -29 -

Figure 2.1 The effect of medium on the c-di-GMP mediated dispersal response of Pseudomonas aeruginsoa PAO1/p $\mathrm{p}_{B A D^{-}} y$ hjH biofilms, carrying the arabinose-inducible phosphodiesterase $y$ hjH, were formed in ABTGC, MHB, TSB and LB for quantification of biofilm formation and dispersal. The amount of biofilm formed (A) and dispersed upon arabinose addition as measured by CV staining ( $\left(\mathrm{Abs}_{590}\right)$, resulting changes to sample turbidity $\left(\mathrm{O} \mathrm{D}_{600}\right)(\mathbf{B})$ following dispersal. Establishment of initial inocula conditions for all further experiments (C) as turbidity vs. CFU/mL and a comparison of induced dispersal as $\mathrm{CFU} / \mathrm{mL}$ (D) of the supernatant above the biofilms for treated and untreated samples in ABTGC and MHB media. Values represent the average of 3 independent experiments, +/- STDEV; ${ }^{* \star}$ : $p$-value $<0.001$ multiple t-test, using the Bonferroni-Dunn method, $\alpha=0.05$. $-44-$

Figure 2.2 MICs $\left(O . D_{600}\right)$ and $M B C s$ as measured using the REMA assay (emission at $590 \mathrm{~nm}$ ) for four antibiotics. MICs (A, C and E) and MBCs (B, D and F) were determined for imipenem against the w.t $P$. aeruginosa $\mathrm{PAO} 1$ strain, $\mathrm{PAO} 1 / \mathrm{p}_{B A D}-y h j H$ and PAO1/pJN105 carrying the empty vector (A and B), while the MIC and MBCs were determined for imipenem \& colistin (C and $\mathbf{D})$ or tobramycin \& azithromycin (E and $\mathbf{F}$ ) for $\mathrm{PAO1} / \mathrm{p}_{B A D}-y$ hjH. All measurements were made following 24-hour exposure at $37^{\circ} \mathrm{C}$. All experiments were performed in triplicate, and the error bars are $+/-$ 
STDEV. Antibiotic concentrations are shown on a $\log _{2}$ scale (thus the control with $0 \mu \mathrm{g} / \mathrm{mL}$ concentration could not be plotted) $46-$

Figure 2.3 Effects of 4-hour arabinose (Ara) induction on 24, 48 and 72-hour grown biofilms of PAO1 strains $\mathrm{p}_{B A D}-y$ hjH, pJN105, and w.t grown in 24-well plates. (A) Remaining biofilm stained by crystal violet (CV) measurement ( $\left.\mathrm{Abs}_{590}\right)$, (B) Turbidity O. $D_{600}$ and (C) $C F U / m L$ of dispersed cells in the sample supernatant. Results were analyzed by two-way ANOVA. Statistically significant differences are given as corresponding p-value, interpretation: ${ }^{* *}<0.01^{* \star *}<0.001^{* * *}<0.0001$. Experiments were performed in triplicates $(\mathrm{N}=3)$ and plotted as average values +/- STDEV ..... 49 .

Figure 2.4 Isobolograms illustrating the relative fractional biofilm eradication concentration (FBEC) values for imipenem (IMI) and tobramycin (TOB). Absolute concentration values $[\mu \mathrm{g} / \mathrm{mL}]$ are indicated at points. Results are the lowest $\sum$ FBEC values from five independent experiments. Combined antibiotic 24-hour treatment against 3-day grown biofilms of strains PAO1/p BAD $_{\text {-yhjH }}(\mathbf{A})$ and PAO1/pJN105 (B) with $(\mathrm{Ara}+)$ or without (Ara-) 4 hours induced dispersal with $1 \%$ arabinose. $-52-$

Figure 2.5 Biofilm c-di-GMP mediated dispersal induced by arabinose enhances the synergistic antimicrobial effect of tobramycin (TOB) combined with imipenem (IMI) against $P$. aeruginosa PAO1/p $\mathrm{p}_{B A D}-y h j H$. Residual bacterial load (CFU/beads) after exposing a 3-day grown biofilms of $P$. aeruginosa $\mathrm{PAO} 1 / \mathrm{p}_{B A D}-y$ hjH $(\mathbf{A})$ or PA01/pJN105 (B) treated (Ara+) or untreated (Ara-) with arabinose $1 \%$ to IMI and TOB alone or in combination with $8 \mu \mathrm{g} / \mathrm{mL}$. The dashed line represents $2 \log _{10}$ reduction in CFU/Bead relative to untreated controls, i.e. eradication of $99 \%$ of bacteria. Results are expressed as the average value of five independent experiments +/- STDEV, $(\mathrm{N}=5)$. $-54-$

Figure 2.6 Confocal Microscopy (CLSM) images (20x objective) (A) of 3-day biofilms of $P$. aeruginosa strains $\mathrm{PAO} 1 / \mathrm{p}_{B A D}-y h j H$ and $\mathrm{PAO} 1 / \mathrm{pJN} 105$ treated with arabinose $1 \%($ Ara + ) or untreated (Ara-) for 4 hours followed by 24 hours exposure to imipenem (IMI), tobramycin (TOB) or combination of IMI + TOB at $8 \mu \mathrm{g} / \mathrm{mL}$ each. Biofilms were stained with Syto9 and propidium iodide prior imaging. Dead and live cells appear red and green, respectively; the scale bar is $20 \mu \mathrm{m}$. Live-dead ratios for biofilms after 24 $\mathrm{h}$ exposure to antibiotics are given (B); Images from five independent experiments $(\mathrm{N}=5)$ were analyzed to determine dead/live ratios for each condition. Results were analyzed using two-way ANOVA ( $p$-value interpretation: ${ }^{* *}$ : $p<0.0001,{ }^{* *}: p<0.001$, $*: p<0.05)$ and plotted as average values +/- STDEV. $-56-$ 
Figure 2.7 Model of proven hypothesis: Inducing dispersal through c-di-GMP depletion enhances the efficacy of combined antibiotic treatment (in red and blue) against mature biofilms. Red bacteria represent dead cells, and green bacteria represent remaining viable cells. Following induced dispersal by overexpression of yhjH PDE enhanced killing was evident, and the ratio of dead/live bacteria in biofilms also increased. $-58-$

Figure 3.1 CFU/Bead count on MHA plates containing $0-64 \mu \mathrm{g} / \mathrm{mL}$ colistin for PAO1/p $\mathrm{p}_{B A D}-y h j H:: G F P$ biofilms grown on $5 \mathrm{~mm}$ beads for $1,2 \& 3$ days in MHB with $0.25 \%$ arabinose $($ Ara + ) or without (Ara-). Results are from three independent experiments and presented as average values +/- STDEV $(\mathrm{N}=3)$. $-74$

Figure 3.2 Example for colony morphologies observed on Congo-red containing agar plates, following 24 hours incubation in $37^{\circ} \mathrm{C}$ and 48 hours at room temperature. In this example a 1-day grown biofilm that was not treated with either arabinose $0.25 \%$ or colistin $8 \mu \mathrm{g} / \mathrm{mL}$ ("Control Ara-"). Serially diluted five times (CFU x $10^{6}$ final), was plated onto Congo-red agar plate supplemented with $4 \mu \mathrm{g} / \mathrm{mL}$ colistin. -76 -

Figure 3.3 Development of population variants in $\log _{10}(\mathrm{CFU} / \mathrm{Bead}$ ) (Smooth, Spreader \& Small) for 1-3 days of growth, in different growth conditions: MHB only (Control), $8 \mu \mathrm{g} / \mathrm{mL}$ colistin, with or without $0.25 \%$ arabinose (Ara+ / Ara-). X-axis represents colistin concentration in plates $[\mu \mathrm{g} / \mathrm{mL}]$. Experiments conducted in quadruplicates, $(\mathrm{N}=4)$ expressed as the average value of four independent experiments +/- STDEV. -77 .

Figure 3.4 Example motility patterns of selected Smooth, Spreader and Small colony variants originating from the parent PAO1/p ${ }_{B A D}-y h j H:: G F P$. Swimming (Left) and Swarming (Right) agar assays have been used to evaluate the motility phenotypes of selected variants, here two examples of each morphotype have been shown. The surface coverage $\left(\mathrm{mm}^{2}\right)$ has been analyzed using ImageJ software on images acquired in gel imaging station utilizing expressed GFP fluorescence. $-80-$

Figure 3.5 Preliminary development of population variants results as $\log _{10}$ (CFU/Bead) (Smooth, Spreader \& Small) for 3-12 days of growth, in different growth conditions: MHB only (Control), $8 \mu \mathrm{g} / \mathrm{mL}$ colistin, with or without $0.25 \%$ arabinose (Ara+ / Ara-). X-axis represents colistin concentration in plates $[\mu \mathrm{g} / \mathrm{mL}]$. For 7-day and 12-day grown biofilms, only one experiment was conducted once with technical duplicates. $82-$ 
Figure 4.1 Differences in growth patterns between colistin resistant $P$.aeruginosa

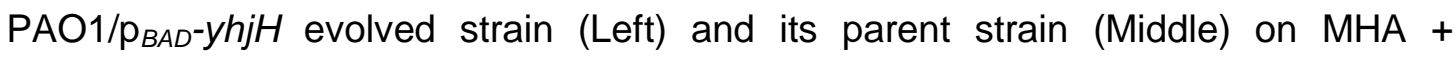
gentamycin $60 \mu \mathrm{g} / \mathrm{mL}$ plates and in $\mathrm{MHB}+$ gentamycin $30 \mu \mathrm{g} / \mathrm{mL}$ (Right) after overnight growth in $37^{\circ} \mathrm{C}$. $-102-$

Figure 4.2 The two strains exhibit different growth kinetics, when grown in MHB media at $37^{\circ} \mathrm{C}$ with shaking at $200 \mathrm{rpm}$. Growth was measured as an increase in turbidity at $O . D_{600}$ in 24-well plates; experiments have been conducted in quadruplicates $(\mathrm{N}=3)$. $-103-$

Figure 4.3 Differences in swimming (Top) and swarming (Bottom) motility phenotypes between the $\mathrm{PAO} 1 / \mathrm{p}_{B A D}-y h j H$ parent (left) and resistant strain (right) $-104-$

Figure 4.4 PAP of parent PAO1/p $\mathrm{p}_{B A D^{-}} y h j H$ strain and its colistin resistant derivative. O.N. culture and serial dilutions plated on MHA containing 0-64 $\mu \mathrm{g} / \mathrm{mL}$ colistin. Results calculated as $\log _{10}(\mathrm{CFU} / \mathrm{mL})$. The experiment was conducted in triplicates plotted as average values +/- STDEV $(\mathrm{N}=3)$. -107 -

Figure 4.5 Analysis of RNA sequencing data, of 280 differently expressed genes exhibiting a >2 absolute fold-change (adjusted p-value $<0.05$ ) generating (A) principal component analysis (PCA) of triplicates of each strain, parent and colistin resistant and (B) expression heatmap in each strain (Red, upregulation; blue, downregulation), (C) pie chart of gene ontology (GO) categorized by biological function (PseudoCAP) $-110-$

Figure 4.6 CLSM images (20x objective) (A) of 3-day-old biofilms of PAO1/p $\mathrm{p}_{B A D}-y$ hjH colistin resistant (Top) and parent (Bottom) strains treated with arabinose 1\% (Ara+) or untreated (Ara-) for 4 hours followed by 24 hours exposure to colistin at $8 \mu \mathrm{g} / \mathrm{ml}$ or not (Control). Biofilms were stained with Syto9 and DAPI prior imaging. Cells appear green; total DNA appears blue and cyan when co-localized. Scale bar is $20 \mu \mathrm{m}$. (B) Bacterial load and total DNA after 24-hour antibiotic exposure reported as DAPI/Syto9. Images from five independent experiments $(\mathrm{N}=5)$ were analyzed to determine dead/live ratios for each condition, presented as average value +/- STDEV. Results analyzed to evaluate eDNA/Cells ratios for each condition in both strains. Statistical significance analysis conducted using two-way ANOVA, corrected by Sidak hypothesis testing ( $p$-value interpretation: $\left.{ }^{*}<0.05\right)$. $-115-$ 
Figure 4.7 C-di-GMP mediated biofilm dispersal induced by arabinose allows synergistic antimicrobial effect for colistin combined with vancomycin against the resistant $\mathrm{PAO} 1 / \mathrm{p}_{B A D^{-}} y$ hjH but not its parent strain. Residual bacterial load $\log _{10}$ (CFU/Bead) after exposing 1-day-grown biofilms of both strains. Biofilms were treated (Ara+) or untreated (Ara-) with 1\% arabinose for 4 hours and then exposed to colistin $(8 \mu \mathrm{g} / \mathrm{mL})$ or vancomycin $(64 \mu \mathrm{g} / \mathrm{mL})$ alone or in combination (at time=0). Experiments conducted in quadruplicates $(\mathrm{N}=4)$. $-116-$

Figure 4.8 Schematic description of the combined effect of biofilm dispersal and the "seesaw effect". 1a. Colistin exposure and sub-optimal concentrations result in enrichment of the colistin resistant sub-population. 1b. Resistant subpopulations become more susceptible to vancomycin. 2a. Biofilm dispersal is crucial to enable penetration of both drugs through the EPS. 2b. Combined vancomycin and colistin treatment following dispersal has the potential for bactericidal effect. 127 


\section{List of Tables}

Table 2.1 Evaluation of MIC and MBC of 4 different antibiotics for PAO1/p $\mathrm{p}_{B A D}-y$ hjH by different assays and comparison to literature results $-47-$

Table 3.1 Phenotypic properties of 20 selected colonies from the 3 different morphotype categories picked from $32 \mu \mathrm{g} / \mathrm{mL}$ colistin plates. -79 -

Table 4.1 Phenotypic differences between parent and colistin resistant derivative PAO1/p $\mathrm{p}_{B A D^{-}}$hhjH strains $-105-$

Table 4.2 MIC values for the PAO1/ $\mathrm{p}_{B A D^{-}} y$ hjH parent and colistin resistant strains 106

Table 4.3 Top hits from the pairwise comparison, detected variations between the two PAO1/p $p_{B A D}-y h j H$ strains at $100 \%$ frequency. 108

Table 4.4 MBIC and MBEC range values for both PAO1/p $\mathrm{p}_{B A D}-y$ hjH strains, 1-day grown biofilms. -112 -

Supplementary Table 1 SNP between parent and colistin resistant PAO1/P ${ }_{B A D^{-}} y h j H$ by pairwise comparison of whole genome sequencing. $-159-$

Supplementary Table 2 Differentialy upregulated genes in the colistin resistant $\mathrm{PAO1} / \mathrm{P}_{B A D^{-}} y$ hjH strain relative to its parent strain -160 -

Supplementary Table 3 Differentialy downregulated genes in the colistin resistant

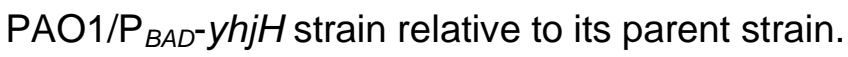
$-163-$ 


\section{Abbreviations}

A. baumannii

$\mathrm{AHL}$

Al

AMR

A.U

AUC

${ }^{\circ} \mathrm{C}$

$\mathrm{C}_{\max }$

CBD

CDC

c-di-GMP

CF

CFU

CV

CLSI

CLSM

DGC

DTU

E. coli

eDNA

EPS

FBEC

FIC

g

GFP

$\mathrm{Gm}^{\mathrm{r}}$

k

$\mathrm{kHz}$
Acinetobacter baumannii

acylhomoserine lactone

autoinducers

antimicrobial resistance

arbitrary units

area under curve

degrees Celsius

maximum serum concentration

Calgary Biofilm Pin Lid Device

Center for Disease Control and Prevention

3',5'-Cyclic diguanylic acid

Cystic Fibrosis

colony forming units

Crystal Violet

Clinical Standards Institute

confocal laser scanning microscopy

diguanylate cyclase

Technical University of Denmark

Escherichia coli

extracellular DNA

extracellular polymeric substances

fractional biofilm eradication concentration

fractional inhibitory concentration

gram

Green Fluorescent Protein

Gm resistance

kilo $\left(10^{3}\right)$

kilohertz (1000 cycles per second) 


\begin{tabular}{|c|c|}
\hline K. pneumonia & Klebsiella pneumonia \\
\hline L & liter \\
\hline LB & Lysogeny Broth \\
\hline LPS & lipopolysaccharides \\
\hline$\mu$ & micro $\left(10^{-6}\right)$ \\
\hline $\mathrm{m}$ & meter \\
\hline $\mathrm{m}$ & milli $\left(10^{-3}\right)$ \\
\hline M & molar (mol per liter) \\
\hline MDR & multi-drug resistance \\
\hline MIC & minimal inhibitory concentration \\
\hline MHB & Mueller-Hinton Broth II \\
\hline MHA & Mueller-Hinton Agar II \\
\hline MBC & minimal bactericidal concentration \\
\hline MBIC & minimal biofilm inhibitory concentration \\
\hline MBEC & minimal biofilm eradication concentration \\
\hline MDK & minimal duration for killing \\
\hline MNV & Multi-nucleotide Variation \\
\hline $\mathrm{mol}$ & mole (amount of substance) \\
\hline $\mathrm{mw}$ & molecular weight \\
\hline $\mathrm{n}$ & nano $\left(10^{-9}\right)$ \\
\hline $\mathrm{NADH}$ & Nicotinamide-adenine-dinucleotide \\
\hline O.D & optical density \\
\hline O.N. & overnight \\
\hline $\mathrm{p}$ & pico $\left(10^{-12}\right)$ \\
\hline$P$. aeruginosa & Pseudomonas aeruginosa \\
\hline PAP & population analysis profile \\
\hline PBP & penicillin-binding protein \\
\hline PDE & phosphodiesterase \\
\hline QS & quorum sensing \\
\hline
\end{tabular}


REMA

rpm

S. aureus

SCV

SNV

STDEV

UV

$\mathrm{vol} / \mathrm{vol}$

$\mathrm{WHO}$

w.t

$\mathrm{w} / \mathrm{v}$
Resazurin Microtiter Assay

revolutions per minute

Staphylococcus aureus

Small-colony variant

Single-nucleotide Variation

Standard Deviation

ultra violet

volume per volume

World Health Organization

wild-type

weight per volume 


\section{Chapter 1: Literature Review}

\subsection{Antimicrobial Resistance (AMR)}

Since the initial discovery of penicillin by Alexander Fleming in the 1920s, antibiotics have been the primary treatment agents for acute and chronic infections caused by bacteria (Huttner, Harbarth et al. 2013). The development of new antibiotics has substantially slowed down in recent decades (World Health Organization 2016). The Review on Antimicrobial Resistance (AMR) commissioned in July 2014 by the United Kingdom (O'Neill 2014) has estimated that in the near future, AMR and microbial infections will surpass cancer in death toll and costs, over 10 million deaths per year and at least $\$ 100$ trillion of the British gross national product. Those conclusions were recently supported by the World Health Organization (WHO) who has called for researchers, health care professionals and officials to collaborate and focus on global prevention and stewardship programs (World Health Organization 2016).

The frequent administration and overuse both for human health and agriculture have been associated with the increased spread of AMR over recent decades. It is estimated that the prevalence of acquired hospital infections is in the range of $1-10 \%$ of all patients admitted and that this is likely to be higher in developing countries (Allegranzi, Nejad et al. 2010). Estimated costs for AMR related infections in the US alone are in the range of at least 55 billion USD when parameters such as prolonged length of stay, more intensive 
antibiotic treatment regimens utilized, additional complications and higher mortality rates are calculated (Smith and Coast 2012).

AMR phenotypes can be divided into three main categories: Resistance, Tolerance, and Persistence (Brauner, Fridman et al. 2016). Resistant bacteria are bacteria that express or acquire new genes that allow them to sustain increasing concentrations of antibiotics; such as enzymes that actively degrade antimicrobials, scaffold proteins that are a "barrier" for antibiotics or develop resistance through mutation of antibiotic targets (specific mechanisms in the next section). Tolerance, on the other hand, allows subpopulations to withstand transient exposures to high antibiotic concentrations. The ability to form biofilms has been linked to increased tolerance of bacteria.

Bacteria exhibit a complex life cycle, switching from planktonic cells to an aggregated, sessile, often surface attached form, called a biofilm, under different conditions. Approximatively $60-80 \%$ of infections are biofilmassociated and can be categorized into two groups: 1) Medical device-related infections (such as catheters, stents) \& 2) infections of host tissue (skin, lungs or heart) (Høiby, Bjarnsholt et al. 2015). These infections are defined as "hard to treat", usually chronic, and represent a therapeutic challenge for clinicians, especially when they involve multiple species, multidrug-resistant isolates and when the site of infection is difficult to reach (Gellatly and Hancock 2013). In biofilms, the extracellular matrix represents a diffusion barrier to antimicrobials resulting in the delivery of sub-inhibitory concentrations of the antibiotics to the cells. Bacteria also exist in different metabolic states, which ultimately affect their susceptibility to antimicrobial agents. Antimicrobial combination therapy 
has been shown to exhibit higher efficacy in treating biofilms (Černohorská and Votava 2008, Herrmann, Yang et al. 2010, Hengzhuang, Wu et al. 2011). Nonetheless, it harbors some limitations such as treatment durations, which can remain long and therefore, favorable for the development of drug resistance or side effects.

Persistence occurs when a small portion $(<0.01 \%)$ of the bacterial population becomes metabolically dormant (Brauner, Fridman et al. 2016). This random, subpopulation of cells is therefore insensitive to the antibiotics and survives. Persistence is a non-heritable trait, induced by various environmental stresses, such as nutrient depletion. Persistent cells exist as part of the population, were considered until recently as viable but non-culturable, and when the antibiotic stress is removed this cell population regrows as normal cells (Kim, Chowdhury et al. 2018).

\subsection{Antibiotic Resistance Mechanisms}

As described above, bacteria have a number of strategies that enable them to survive the antibiotic pressure. The opportunistic pathogen Pseudomonas aeruginosa ( $P$. aeruginosa) is an example of such pathogens that is intrinsically resistant to several groups of antimicrobials. This bacterium can augment resistance with tolerance and persistence mechanisms and also can acquire resistance through horizontal gene transfer (Livermore 2002). The following sections present general bacterial resistance mechanisms to different classes of antibiotics, which are widely distributed in $P$. aeruginosa. 


\section{Carbapenems}

Carbapenem antimicrobials belong to the broader antibiotic group of $\beta$-lactams (Zhanel, Wiebe et al. 2007). These compounds have structural similarity to penicillin and cephalosporin antibiotics. All $\beta$-lactams are made of a cyclic ring structure motif, and their primary target is the penicillin-binding protein (PBP) transpeptidase enzyme family, which governs the synthesis of the bacterial cell wall (Morita, Tomida et al. 2013). Due to low oral availability, carbapenems are best administered intravenously, and due to their broad-spectrum activity, they are often used to prevent nosocomial infections (Rello 2007). The two carbapenem agents frequently administered to treat $P$. aeruginosa are imipenem and meropenem.

The target sites for carbapenems are adjacent to the bacterial inner membrane, at the intermembrane space, and cytosol (Chen, Zhang et al. 2016). Carbapenems need to pass the selective barrier of the outer cell membrane, through porin channels, and more specifically the complex OprD (Amin, Giske et al. 2005). Variants of $P$. aeruginosa expressing lower levels of this porin exhibit slower growth rates but also become tolerant to carbapenems. $P$. aeruginosa, like many other organisms, can express $\beta$-lactamase enzymes, which actively degrade $\beta$-lactam antibiotics; these enzymes can have very different modes of action and can exist in several copies in bacteria. Most $\beta$-lactamases are inefficient in degrading carbapenems, though continued exposure can lead to overexpression of $\beta$-lactamases, especially $\mathrm{AmpC}$, and also mutated enzymes with better capabilities have been reported (Wolter and Lister 2013). Some bacteria express multidrug efflux pumps, such as MexAB-OprM, MexCD-OprJ, 
encoded by $P$. aeruginosa (Okamoto, Gotoh et al. 2002). Mutations to the PBPs, targets of the carbapenems, have been shown to be heterogenic, either culminate in mutations to targets themselves or changes in the expression profiles of the genes PBP1-4 depending on isolates (Moyá, Beceiro et al. 2012).

\section{$\underline{\text { Aminoglycosides }}$}

Aminoglycosides are a group of antibiotics that have several sugar motifs bound to amino groups through the anomeric carbons (Forge and Schacht 2000). The targets of these compounds are the $30 \mathrm{~S}$ small ribosomal units of bacteria, causing inhibition of protein synthesis. The most common aminoglycosides administered for $P$. aeruginosa infections are streptomycin, kanamycin, neomycin, and gentamicin, although tobramycin displays the most significant killing potential of the aminoglycoside antibiotics. The high tolerance of $P$. aeruginosa biofilm to tobramycin has been known since its introduction to treatment (Nickel, Ruseska et al. 1985).

Resistance can be mediated by enzymes that modify the aminoglycosides: aminoglycoside phosphoryl-transferase, aminoglycoside acetyl-transferase, and amino-glycoside nucleotidyltransferase (Poole 2005). The principal mechanism of tolerance employed by $P$. aeruginosa is impermeability and active transport by multidrug efflux pumps such as MexXY-OprM (WestbrockWadman, Sherman et al. 1999, Bulitta, Ly et al. 2015). Furthermore, since there is a need for binding with the lipopolysaccharides (LPS) of the outer membrane of the cell before penetration, the additional potential for resistance exists (Schniederjans, Koska et al. 2017). It has been shown that mutations 
that lead to alterations of the LPS lead to higher resistance in $P$. aeruginosa (Schurek, Marr et al. 2008, Krahn, Gilmour et al. 2012).

\section{Polymyxins}

The polymyxin antibiotic group comprises molecules with a cyclic polypeptide motif, bound to a fatty acid moiety (Storm, Rosenthal et al. 1977), a lipopeptide. The two members of this group that are frequently administered to treat $P$. aeruginosa infections are Polymyxin $\mathrm{B}$ and Polymyxin $\mathrm{E}$, the latter commercially known as Colistin. Polymyxins act via binding of LPS by the polypeptide motif and bacterial membrane disruption by the fatty acid chain to cause lysis (Biswas, Brunel et al. 2012). Polymyxins attack bacterial cells regardless of metabolic state (Breidenstein, de la Fuente-Núñez et al. 2011). Thus colistin is potentially effective against non-dividing cells and persister cells (Cui, Niu et al. 2016).

The primary mechanisms of resistance are modifications to the lipid- $A$ lipopolysaccharide, the main constituent of the outer monolayer of the bacterial outer membrane. The PhoP-PhoQ and PmrA-PmrB two-component systems (TCS) have been found to regulate the arn operon (Breidenstein, de la Fuente-Núñez et al. 2011). Enzymes encoded in this operon constitute Lipid-A modifications such as additions of 4-amino-L-arabinose or phosphorethanolamine (Fernández, Álvarez-Ortega et al. 2013). These changes reduce the overall net negative charge of the membrane, limiting the reaction with polymyxins (Lee, Park et al. 2016). Polymyxins displace $\mathrm{Mg}^{+2}$ and $\mathrm{Ca}^{+2}$ ions from the LPS membrane, before fully binding to Lipid-A, and their activity depends on the concentration of these divalent ions and $\mathrm{pH}$ (Conly and 
Johnston 2006). The PhoP-PhoQ system is expressed together in one operon with the protein OprH that binds directly to the LPS and assists in its stabilization (Lim, Ly et al. 2010).

\subsection{Biofilm-associated tolerance}

It is estimated that about two-thirds of hospital-acquired infections are biofilmrelated (Høiby, Bjarnsholt et al. 2010). Biofilms are associated with medical device-related infections as well as chronic infections that are recalcitrant to clearance by the host immune system or antibiotic therapy (Lewis 2008). Indeed, biofilms are associated with a general increase in the survival of bacteria when challenged with extreme environmental conditions, immune system (e.g., reactive oxygen) or antibiotic stresses. For example, it has been reported that $100-1000$ fold higher concentrations of antibiotics are required to kill biofilm bacteria compared to planktonic cells (Spoering and Lewis 2001).

The increased tolerance of bacteria within biofilms appears to be multifactorial (Høiby, Bjarnsholt et al. 2010). Bacteria have been shown to be physiologically stratified in biofilms, where cells on the surface tend to be more active while bacteria within the interior may be less active, dormant or undergoing anaerobic metabolism (Williamson, Richards et al. 2012). Thus, the cells in these different states may respond to antibiotics differently, if, for example, the antibiotic mechanism involves attacking metabolically active cells under aerobic conditions (Martínez and Rojo 2011). Biofilm bacteria excrete and form an intricate, heterogeneous but organized matrix composed mainly of polysaccharides, proteins, and extracellular DNA (eDNA) cumulatively termed 
"extracellular polymeric substances" (EPS) (Flemming, Wingender et al. 2016). The ratios of "substances" in the EPS differ from species to species and depend on environmental conditions, as well as extracellular queues. Stochastic changes such as threats and stress factors like antibiotics or availability of nutrients and electron acceptors are just some factors that can affect the EPS constituents (Stewart and Franklin 2008).

The EPS has been suggested to lessen penetration of some antibiotics and hence, protects the underlying cells (Yang, Hu et al. 2011). For example, eDNA, which is one of the major EPS components of $P$. aeruginosa biofilms, is negatively charged and thus acts as a passive diffusion barrier to cation antibiotics. Through the chelation of $\mathrm{Mg}^{2+}$ and $\mathrm{Ca}^{2+}$ overproduction of eDNA has been shown to increase antibiotic resistance through activation of the twocomponent systems OprH-PhoP/Q and PmrAB (Mulcahy, Charron-Mazenod et al. 2008, Lewenza 2013). In contrast, the exopolysaccharide Psl is neutrally charged polymer composed of repeats of glucose, mannose and rhamnose, and has been suggested to provide a selective barrier to several antibiotic groups (Billings, Ramirez Millan et al. 2013, Flemming, Neu et al. 2016).

Biofilm formation has been shown to select for genetic variants, such as small colony variants (SCV) of $P$. aeruginosa, which were further shown in-vitro and in-vivo to exhibit higher tolerance to antimicrobials than their ancestors (Kirisits, Prost et al. 2005, Hogardt and Heesemann 2013). SCVs produce large amounts of the exopolysaccharides Psl and Pel while exhibiting reduced motility due to the high intracellular content of c-di-GMP (Starkey, Hickman et al. 2009). The mucoid variants of $P$. aeruginosa were frequently reported to 
predominate following infection, and have been shown to overproduce the alginate polysaccharide (Flemming, Neu et al. 2016). The contribution of alginate to antibiotic resistance is still debated, although the reason lies in the fact that it is mostly exhibited by mature biofilms (Govan and Deretic 1996). Collectively, variants are associated with overproduction of EPS constituents, metabolic heterogeneity of the population, which result in higher tolerance and ultimately manifest higher persistence to antibiotics (Starkey, Hickman et al. 2009). SCVs seem to bear all of the mechanisms mentioned above and have been shown to have higher proportions of persister cells when they result from prolonged antibiotic treatment (Wei, Tarighi et al. 2011). Figure 1.1 symbolizes the multi-factorial recalcitration: heightened antibiotic tolerance, resistance and persistence.



Figure 1.1 A schematic representing tolerance and resistance mechanisms in biofilms, antibiotic penetration (yellow gradient) into the inner levels of biofilm is slowed down due to viscosity and ionic and hydrophobic interactions with EPS constituents (eDNA, Psl). Sub-optimal antibiotic concentration leads to higher expression levels of resistance/tolerance genes in the inner biofilm levels (green); 
and heterogenic local environments in biofilms due to oxygen and nutrient gradients (red gradient). All of the above lead to different metabolic rates and heterogenic gene expression. Antibiotic treatment thus results in partial efficacy; not all cells are killed (red bacteria) some live cells always remain (green bacteria). Finally, the biofilm population harbors higher frequency of persister cells (purple bacteria).

The observations that: 1) many infections are associated with biofilms and that 2) biofilm formation facilitates antibiotic resistance and tolerance - therefore, argue that an understanding of how biofilms form is essential. Further, understanding the regulatory mechanisms and key genes in biofilm formation may identify novel targets to better control biofilms by reversing their inherent, high tolerance to antimicrobials. This literature review will provide a general introduction to the biofilm life cycle, focusing on the Gram-negative bacterium that is the model organism used throughout this thesis, $P$. aeruginosa.

\subsection{Microbial Life on Surfaces}

Biofilm formation is typically described as starting with planktonic, free-living cells migrating to and interacting with surfaces (Flemming, Wingender et al. 2016). Interactions with surfaces as substrata for anchoring are initially unspecific, by means of Van der Walls, electrostatic and hydrophobic interactions (Palmer, Flint et al. 2007). Bacterial surface pili and flagella protein complexes often mediate initial attachment (Klausen, Heydorn et al. 2003). Irreversible attachment is considered the step which is normally cementing the bacteria on the surface, involving repression of the flagellar swimming motility and production of EPS constituents (Ha and O'Toole 2015). 
Type IV pili facilitate irreversible binding, and bacterial surface motility, known as twitching (Rasamiravaka, Labtani et al. 2015). An additional form of social motility in biofilms is swarming, which allows for movement of bacteria on semi-solid surfaces and involves flagella, pili, EPS constituents and biosurfactants (Shrout, Chopp et al. 2006). Motility, surface-bound or freeswimming, drives chemotaxis, while still energetically favorable. Once irreversibly attached, biofilms develop by surface-bound growth and further recruitment of cells from the environment, both contribute to the local establishment of micro-colonies (Høiby, Krogh Johansen et al. 2001).

As bacteria grow by multiplication, if the population is sparse there is more room for growth, and it is mostly flat. As the population density increases, physical constraints cause the newly formed bacteria to push towards the periphery (Sheraton, Yam et al. 2018). Microcolony development can result in the formation of a range of different structures depending on the growth conditions, and motility ( $\mathrm{Ni}$, Yang et al. 2016). For example, in defined media containing succinate, glutamate or citrate as a sole carbon source, on surfaces which permit swarming, biofilms first appear to develop flat structures (Shrout, Chopp et al. 2006). Biofilm growth and maturation are a function of the available nutrients, physical conditions and cell-to-cell signaling within the biofilm. In continuous culture conditions, elaborate microstructures, resembling mushroom shapes were discovered in $P$. aeruginosa biofilms, on glucosecontaining media; with environmental heterogeneity driving the bacteria cells to high differentiation (Garrett, Bhakoo et al. 2008). Both non-motile and motile bacteria contribute to the structure, biofilms continuously aggregate, disperse 
and recruit planktonic bacteria from the medium (Klausen, Heydorn et al. 2003).

In a similar manner to planktonic growth kinetics, biofilm's exponential growth persists until nutrients (such as carbon and nitrogen sources) have been depleted from the surrounding environment. Whereas planktonic bacteria acquire nutrients in a "foraging" behavior, biofilm cells are sessile and experience higher metabolic heterogeneity. This heterogeneity in biofilm is due to the impact of neighboring cell metabolism, local internal gradients, nutrient, oxygen or additional electron acceptors or any other related factors within the biofilm. This principle contributes to heterogeneity of differential gene expression between every cell in the biofilm. For example, internal biofilm cells might perform anaerobic fermentation, while external cells in the periphery perform aerobic respiration, and all are sometimes part of the same colony (Stewart and Franklin 2008, Wessel, Arshad et al. 2014). Bacteria in the colony periphery grow more rapidly, versus cells in the center that have stationary growth. Cells near the substratum are attached more firmly, while cells in the outer layers are more freely detached. Recently, a novel in-silico biofilm growth development simulation showed that chemotaxis has a role in the shaping of $P$. aeruginosa biofilms, which was confirmed in-vitro (Sheraton, Yam et al. 2018). P. aeruginosa biofilm development is a constant interplay between the production of different EPS constituents, which are affected by changing environmental flow rates and nutrient availability, different secondary structures such as streamers have been shown to develop under heterogeneous conditions (Nadell, Ricaurte et al. 2017). 
It has been established that bacterial cell-to-cell signaling, quorum sensing (QS), is a critical process for the continued development of the biofilm (Miller and Bassler 2001). QS is the coordinated action of the bacterial population, by secretion of small specific signaling molecules, termed autoinducers (AI). As Al concentration increases locally, it cascades in a complete change of the bacterial gene expression, allowing microcolonies to act in unison, at a specific density, the quorum ( $\mathrm{Li}$, Li et al. 2015). Three central QS mechanisms that control $P$. aeruginosa biofilm formation are well investigated: las, $r h l$ and $p q s$ (Lee and Zhang 2015). The LasR regulator binds acyl-homoserine-lactone $(\mathrm{AHL})$ activating expression of the operon, the $\mathrm{AHL}$ molecule family is a predominant Al in many bacteria (Lade, Paul et al. 2014). In $P$. aeruginosa las operon controls a network of reactions, for example, cascading in higher expression of $T p b A B$ which negatively controls Pel production and positively controls Psl and eDNA production (Wei and Ma 2013). It was previously shown that a las/ mutant of $P$. aeruginosa makes a flat, undifferentiated biofilm that is less stable than the wild-type (w.t) biofilm (Sakuragi and Kolter 2007). The $r h l$ and pqs operons QS systems control the production of the virulence factors rhamnolipid and pyocyanin, respectively; these virulence markers have been shown to be lethal to host immune cells, as well as perturbed epithelial cell tissues that provide a substrate for biofilm formation (Jakobsen, TolkerNielsen et al. 2017). QS is associated with the increased tolerance of the biofilm to antimicrobials (Tan, Chua et al. 2013); In recent years there exists a paradigm shift in developing novel antimicrobials, the focus is on the repression of bacterial QS, or Al production (Sintim, Smith et al. 2010). Synthetic Al molecules, QS inhibitors, and antagonists to receptors which take 
part in QS are being tested as novel antimicrobials (Beloin, Renard et al. 2014). QS inhibitors of the las and $r h /$ operons have been shown to impair bacterial motility, virulence, antibiotic resistance and biofilm formation. The use of several antagonists to these QS systems culminates in the protection of the innate immune system and provides potential synergy with antibiotics (Jakobsen, Tolker-Nielsen et al. 2017). These data suggest that interfering with the biofilm developmental program can be exploited to reduce or control infections by $P$. aeruginosa.

\subsection{Biofilm dispersal}

Mature biofilms complete the life-cycle where they disperse back into freeliving planktonic cells. Dispersal has been reported to be induced in response to changing environmental conditions such as oxygen, $\mathrm{pH}$, carbon, and nitrogen, or micronutrient concentrations (Guilhen, Forestier et al. 2017). Bacterial biofilms can be removed due to mechanical forces, or if shear forces are applied, and this process is referred to as sloughing. "Active Biofilm dispersal", on the other hand, has been found to require energy and is typically mediated by regulatory events upon sensing of changing environmental cues (McDougald, Rice et al. 2011, Fleming and Rumbaugh 2017) (Figure 1.2). EPS degrading enzymes, either self-produced or exogenously added have been found to induce dispersal from biofilms. The spatial differentiation of bacteria in biofilms, especially in terms of motility is crucial to the dispersal process and thus, QS systems such as $r h /$ and pqs also play an active role in the process (Kim and Lee 2016). 


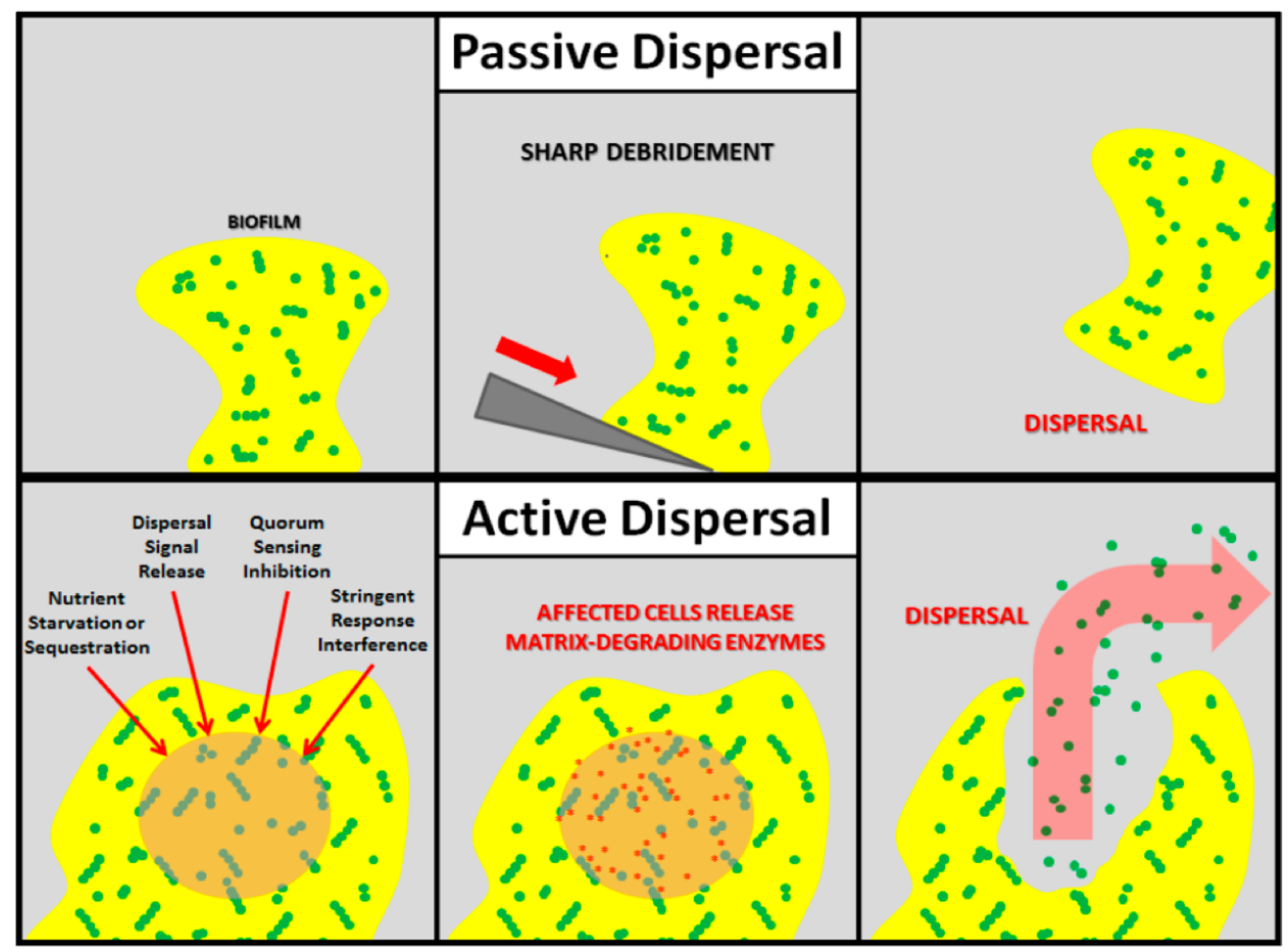

Figure 1.2 A scheme representing passive and active dispersal of bacterial biofilms. Passive dispersal (Top) can be caused by external forces such as shearing while active dispersal (Bottom) is the result of different signal transduction cascades, usually involves enzymes that degrade components of the EPS matrix (Figure taken from: (Fleming and Rumbaugh 2017).

Many of the genetic responses that trigger dispersal are mediated through the secondary messenger, 3',5'-cyclic diguanylic acid (c-di-GMP) (Ha and O'Toole 2015). Secondary messengers relay extracellular cues or signals to the cytoplasmic space, and where catalytic proteins can be activated to synthesize or degrade the intercellular messengers. For many Gram-negative bacteria, the concentration of c-di-GMP controls the mechanism governing the "lifestyle transition" from planktonic to biofilm. Generally, higher intracellular levels of c-di-GMP enhance biofilm formation, while low c-di-GMP levels are associated with dispersal from biofilms or planktonic cells. It is estimated that biofilm bacteria have $75-110$ pmol c-di-GMP per mg total protein in the cell extract, 
while planktonic bacteria have less than 30 pmol c-di-GMP per mg in the cell extract (Valentini and Filloux 2016). C-di-GMP is produced from GTP by diguanylate cyclases (DGCs) and degraded by phosphodiesterases (PDEs).

Dispersed bacteria are distinct in terms of gene expression from biofilm cells and planktonic cells, but following a certain duration of time, they can regain motility, become planktonic, and the cycle begins anew (Chua, Yang et al. 2017). The biofilm life-cycle is usually depicted as a linear development from attachment, to maturation, and then to dispersal, however, it is a cyclic process, with portions of the bacterial cell population existing in all states at the same time in any system, as demonstrated in figure 1.3. Most bacteria that form biofilms have the ability to actively disperse them, as a mechanism that allows recolonization in new niches (Guilhen, Forestier et al. 2017).

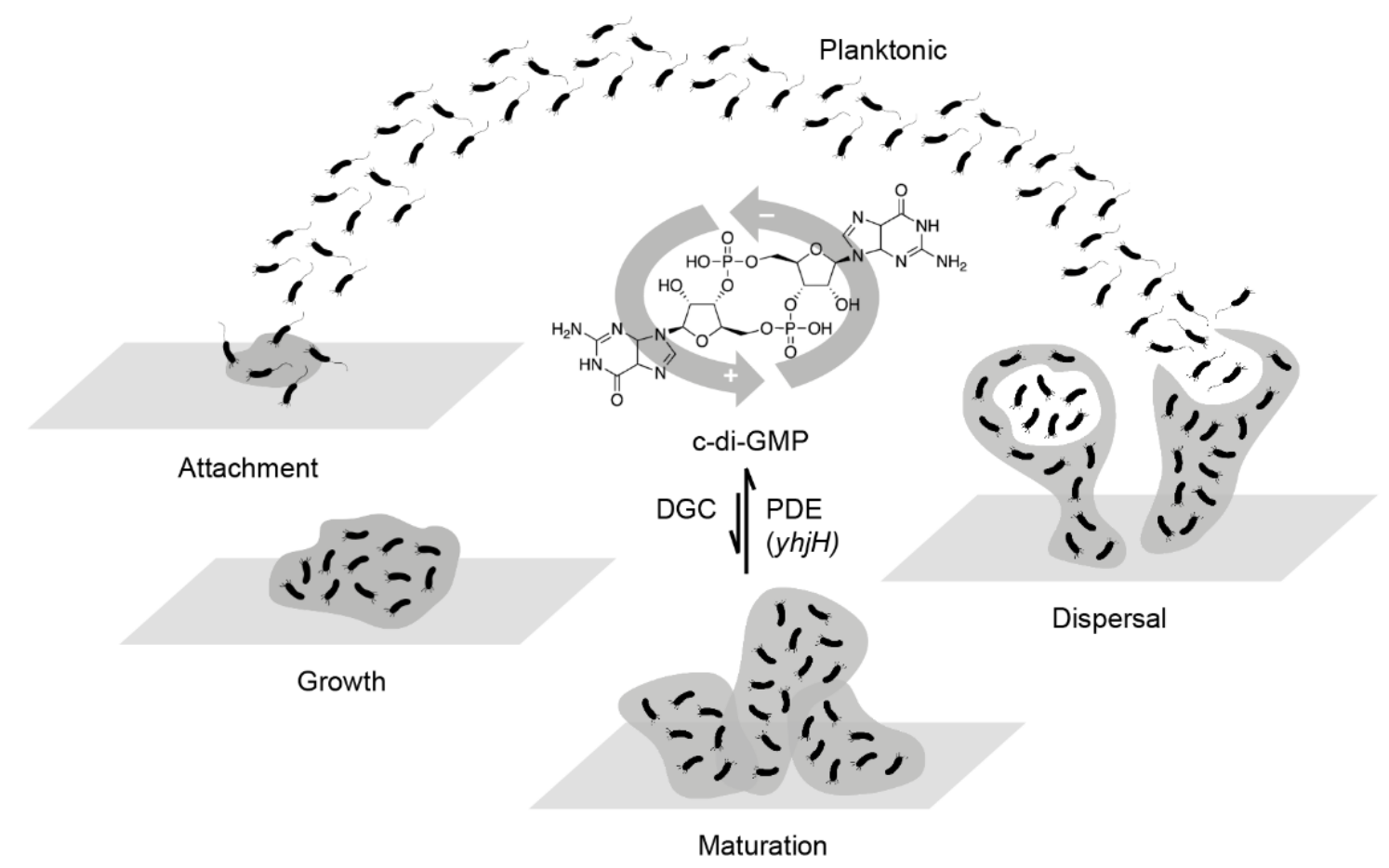

Figure 1.3 The biofilm life-cycle under positive control by the intracellular c-di-GMP concentration. C-di-GMP is the product of DGC enzyme family and degraded by the 
PDE enzyme family. The image shows the correlation between high c-di-GMP (more DGC activity) and biofilm growth and low concentrations (more PDE activity) and dispersal. The P.aeruginosa construct used throughout this thesis overexpresses the $\mathrm{PDE}$ YhjH when induced, thus facilitating higher levels of biofilm dispersal.

Bacteria have been shown to alter PDE and DGC activity in response to environmental cues to modulate biofilm development. For example, it has been shown that biofilms can be dispersed by exposure to nitric oxide (NO) and that perception of this cue triggers a decrease in intracellular c-di-GMP content, resulting in changes in gene expression associated with the conversion of the biofilm cells into planktonic cells (Figure 1.4). Bacteria appear to have evolved the ability to sense and respond to NO. Some bacteria express proteins with PAS domains that bind NO (In $P$. aeruginosa by affecting the activity of chemotaxis regulator bdIA), leading to PDE activation, eventually lowering intercellular c-di-GMP concentrations and resulting in biofilm dispersal (Petrova and Sauer 2012). 




Figure 1.4 A scheme describing the mechanisms of signal transduction which culminate in changes to the intracellular c-di-GMP concentration that result in differential expression in cells and phenotypic changes (Taken from (McDougald, Rice et al. 2011).

Although not extensively used clinically, combinations of dispersal agents and antibiotics have been proven to facilitate higher synergy in-vitro and in-vivo (Kaplan 2010). Antibiotic combinations have been proven to be superior in treating biofilm-related infections (Černohorská and Votava 2008, Dundar and Otkun 2010, Barber, Ireland et al. 2014, Chatterjee, Anju et al. 2016), lowering effective concentrations and targeting different cellular mechanisms. Combined antibiotic therapy following facilitated dispersal has not been shown in previous research, though the potential to further improve efficacy at lower concentrations and short treatment durations is a promising option for new therapeutic strategies. 


\subsection{P. aeruginosa as a Model Gram-negative Biofilm Former}

Pseudomonas is a ubiquitous, aerobic Gram-negative genus (Frangipani, Slaveykova et al. 2008). It can respire oxygen at concentrations as low as $3 \mu \mathrm{M}$ dissolved oxygen or in the absence of oxygen can respire using nitrate as an electron acceptor (Alvarez-Ortega and Harwood 2007). It is found in moist environments such as soils, lakes, and most human-made environments as well as various host microbiomes in mammals, fish, and plants. The genus was initially identified in 1882 by Gessard and termed by Migula in 1894 as Pseudo ("false") monas ("single-celled organism"), indicating that it is unicellular (Lister, Wolter et al. 2009). The identified specie of the genus, aeruginosa (meaning "of blue-green color", the color of rust) has later emerged as one of the key model organisms for the study of biofilm formation.

$P$. aeruginosa is considered to be a ubiquitous nosocomial pathogen and ranks as the fifth of overall frequent hospital-acquired pathogens (Wolter and Lister 2013). It is associated with infections of airways, wounds, medical devices such as catheters and joint replacements, burns, hospital equipment (Deretic 2000) (Figure 1.5). 
The ubiquitous Pseudomonas aeruginosa is also...
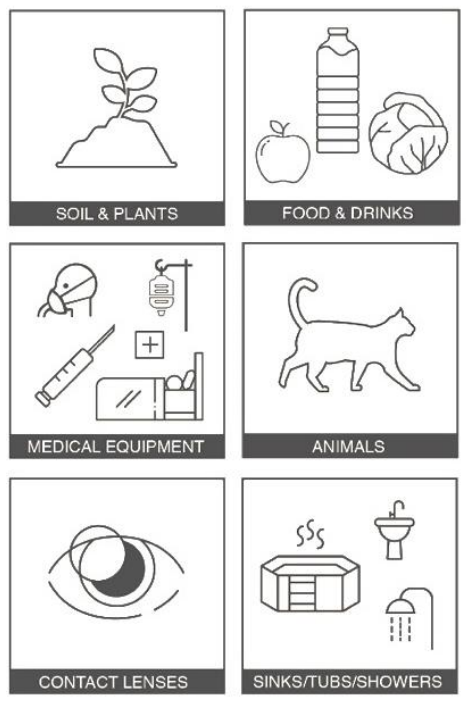

...Pseudomonas aeruginosa, the opportunistic pathogen.
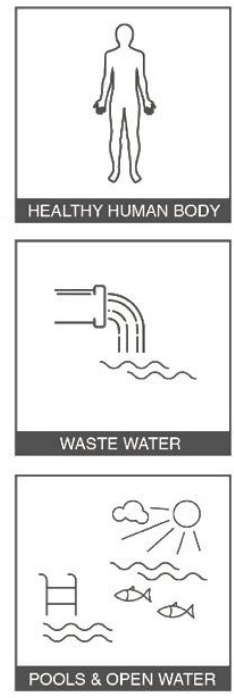

Figure 1.5 $P$. aeruginosa is a versatile organism which exists in many environments, including the human body. When a patient's immune system is compromised, the opportunistic $P$. aeruginosa can be found in various related infections. Images are taken from https://thenounproject.com under creative commons license.

The bacterium can be part of non-sterile body sites, such as the skin, mouth and nostril flora; and would be considered benign, or commensal, merely colonizing the surface. It is regarded as an "opportunistic pathogen" and can penetrate the internal layers of that organ, through wounds, or to the inner parts of the airways and lungs for example, through initial attachment facilitating a biofilm and leading to chronic infection (Wagner, Sommer et al. 2016) (Figure 1.6). 




Figure 1.6 Examples of chronic infections predominated by $P$. aeruginosa biofilm. Scanning electron microscope (images.fineartamerica.com), contact lens-related eye infection (www.honolulugeclinic.com), infected lung tissue of cystic fibrosis patient (microbewiki. keynon.edu), urinary tract infection (jucing-for-health.com), neonatal and pediatric-related infection (dermaamin.com), chronic wound infection (www.medetec.co.uk), folliculitis skin related infection (callisto.ggsrv.com), nail infection (www.danderm-pdv.is.kkk.dk). 


\subsection{Antibiotic testing strategies}

Given its innate antibiotic resistance and its ability to form biofilms that further protect $P$. aeruginosa during infection, it is clear that further work needs to be done to identify effective treatments to control this important pathogen. However, most therapeutics are tested against planktonic cells and do not address the issue of biofilms. As noted above, biofilms can be up to 1000 fold more resistant to antibiotics and hence, biofilms may need to be considered as part of antibiotic testing. The following sections discuss several approaches to testing antimicrobial agents.

\section{Planktonic bacteria}

For susceptibility testing of $P$. aeruginosa to antibiotics, the standard highthroughput procedures involve broth microdilution techniques in tubes or microtiter plates. The straightforward parameter that can be measured is turbidity of the bacterial suspension. The sample which shows a drop in the optical density (O.D) correlates to a minimal inhibitory concentration (MIC) and can be estimated even with the naked eye; or more accurately, with the use of absorbance measurement using a microplate reader. When using readers the threshold that is usually reported is $\mathrm{MIC}_{50}$ the concentration that leads to $50 \%$ inhibition of growth (Amyes, Miles et al. 1996). MIC allows a relatively quick and easy determination of a concentration range of inhibition, with one major drawback, experimental values depend heavily on experimental conditions (e.g. volume of culture, temperature, antibiotic stock preparations and storage) (Wiegand, Hilpert et al. 2008). Crisscrossed gradients of two antimicrobials can be prepared on a challenge plate, termed "checkerboard". The combined 
treatment can yield a cumulative MIC which is lower than each drug alone, thus exhibiting synergism. Synergy is quantified by the fractional inhibitory concentration (FIC), where the inhibitory concentration of each drug in the combination is divided by its MIC alone, summed FIC values lower than 0.5 are considered synergistic.

The clinical standards institute (CLSI), which systematizes protocols for clinical laboratories worldwide advocates on using the Mueller-Hinton Broth II (MHB) when testing for susceptibility (CLSI 2012). MHB is a rich media based on beef extract broth and casein hydrolysate in physiological $\mathrm{pH}(7.3+/-0.1)$ (Mueller and Hinton 1941). Initially developed for susceptibility testing of specific organisms, MHB was found to be satisfactory for growth of virtually all common aerobic human pathogens in a non-selective manner. MHB contains starch which allows for more efficient absorption of antibiotic intermediates and bacterial toxins, and also contains a defined concentration of $\mathrm{Mg}^{+2}$ and $\mathrm{Ca}^{+2}$ ions which stabilize antibiotics, limit adsorption to plastic, as well as simulate physiological blood concentrations (Koeth, King et al. 2000, CLSI 2012, Bakthavatchalam, Pragasam et al. 2018).

An additional parameter to quantify the efficacy of an antibiotic is the minimal bactericidal concentration $(\mathrm{MBC})$, which is the concentration required to kill at least $99 \%$ of bacteria in solution (Amyes, Miles et al. 1996). When the MBC is $\leq 4 \mathrm{x}$ than MIC, the compound is considered to be bactericidal, and if the MBC is $>4 x$ the MIC, it is bacteriostatic (CLSI 2012). One limitation of the MIC and MBC assays is that they typically require 24 hours or longer to obtain complete results. To address this, alternative methods have been developed. One such 
method involves the dye resazurin (Alamar Blue) a highly water-soluble molecule, which can penetrate cell membranes and is reduced by reaction with Nicotinamide-adenine-dinucleotide (NADH) (Candeias, MacFarlane et al. 1998). A relative short incubation period, low reagent price and the ease of use make this assay a suitable candidate for standard susceptibility testing (Sandberg, Schellmann et al. 2009) (Figure 1.7).

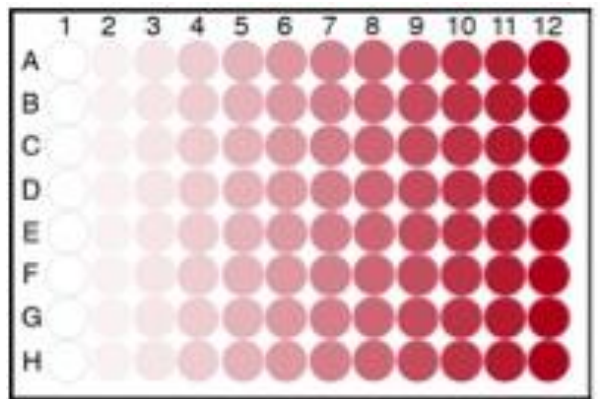

Broth Microdilution

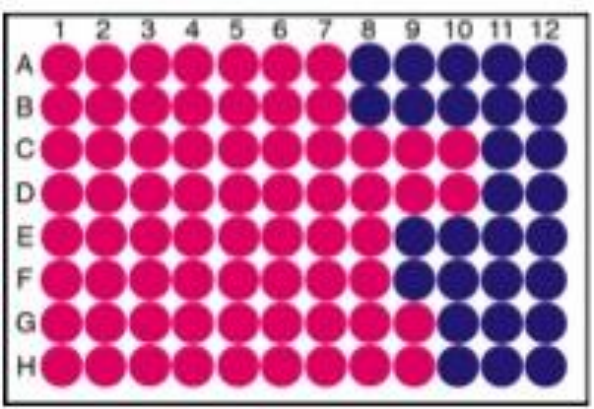

MBC Measurement

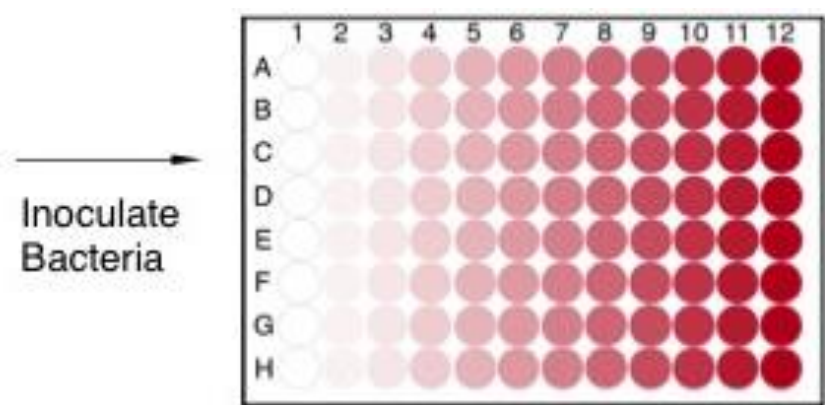

Challenge Plate

Incubate Overnight

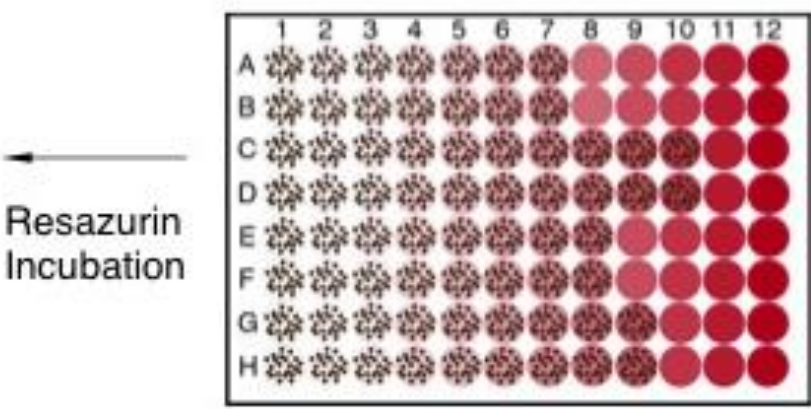

MIC Measurement

Figure 1.7 Microtiter plate broth microdilution method combined to determine MIC, combined with REMA assay to determine MBC. A challenge plate is prepared with a gradient of antibiotic concentrations, in which bacteria are inoculated at $\sim 5 \times 10^{5}$ $\mathrm{CFU} / \mathrm{mL}$ and left overnight. MIC is the concentration that inhibits $50 \%$ of growth. The REMA assay measures MBC: Resazurin in wells which contain active cells becomes reduced (pink) and highly-fluorescent, otherwise remains blue. MBC is the concentration which results in $99 \%$ killing of bacteria. 
The most accurate manner by which MIC/MBC parameters can be assessed involves serial dilutions of sample aliquots following turbidity measurements and enumeration of colony forming units (CFU) following treatments. Though it is considered a more laborious method, it constitutes a test which is less prone to variation assuming each CFU originates from a single bacteria (Hazan, Que et al. 2012).

\section{Biofilm bacteria}

To date, there are no internationally recognized standard procedures to define biofilm antimicrobial susceptibility profile (Malone, Goeres et al. 2017). Currently, biofilm antimicrobial susceptibility is studied using a wide range of different experimental systems, high-throughput or high-content. Two commonly used systems for high-content assessment are the Technical University of Denmark (DTU) biofilm flow cells (Tolker-Nielsen and Sternberg 2011) and the Center for Disease Control and Prevention (CDC) bioreactor (Garey, Vo et al. 2009). Both represent continuous culture systems where the fresh medium is continually supplied to support biofilm growth.

The DTU system has contributed extensively to the understanding of biofilm development usually studied with confocal laser scanning microscopy (CLSM), by continuous time course measurement or at specific intervals. For antibiotic susceptibility, a commonly used technique involves staining bacteria with the "Live/Dead" kit, whereby samples are analyzed by two stains, one that stains the live bacteria population (with intact membranes) and the other that stains dead cells (with compromised membranes). In a research from recent years, the flow cell system was utilized in tandem with isotopic labeling of amino- 
acids to study dynamics of susceptibility of bacteria to colistin in different stages of the biofilm life-cycle (Chua, Yam et al. 2016). The proteomic analysis allowed for translation quantification of specific resistance related enzymes in $P$. aeruginosa under colistin stress, and the effect on other biofilm mechanisms such as virulence.

The CDC biofilm reactor is a system used primarily to examine the efficacy of antimicrobial monotherapies and combinations under conditions, which mimic the kinetics, and dynamics of drugs in the host body. For example, a bioreactor system was used to examine the efficacy of derivatives of novel antimicrobial peptides (Sánchez-Gómez, Ferrer-Espada et al. 2015); because the dynamic properties of the original drug were known, the system was utilized to study $P$. aeruginosa biofilm killing kinetics by its derivatives, thus minimizing the need for in-vivo experiments. This system can also be combined with CLSM and other techniques to examine biofilms at different developmental stages.

Both systems have distinct advantages such as: determining susceptibility under continuous culture conditions which better represent the host environment, and biofilm development under flow conditions, or the versatility of possible experimental setups. However, neither of these systems is amenable to high-throughput screening for a number of reasons: first, they require relatively complicated setups, involving pumps, tubing and glassware; the user has to have a certain level of expertise before meaningful data can be collected. Second, they provide a relatively low number of replicates per experiment, each flow cell contains three separate channels, requiring more 
than one flow cell to be used for each experiment further complicating setup (Heydorn, Ersboll et al. 2000). The bioreactor contains 8 rods, which hold three biofilm samples each; one bioreactor can be used to study one antimicrobial regime at a time, again complicating setups.

A number of high-throughput microtiter based assays are available for evaluating the effect of compounds against biofilms. Biofilms that form in these microtiter plates are typically quantified using crystal violet (CV) as a proxy for the total amount of biofilm present (cells and EPS). CV binds to different negatively charged molecules and especially the peptidoglycan in the bacterial membranes, DNA and EPS constituents (Feoktistova, Geserick et al. 2016).

One significant modification of the microtiter plate biofilm assay is the "Calgary Biofilm Device" (CBD) (Ceri, Olson et al. 1999): a modified plate lid with pegs that hang down into the medium in the wells of the microplate. Biofilms would develop on the pegs as well as in the wells, but for these assays, only the biofilm on the pegs are quantified. A particular advantage of this system is that it quantifies active biofilm formation, where the bacteria have to attach to the pegs rather than quantifying settlement of bacteria from the liquid phase onto the bottom of the plate. Additionally, the lids and associated biofilms can be easily transferred between plates and challenged with antimicrobials. The peg biofilms can then be used to determine the minimal biofilm inhibitory concentration (MBIC). This measure is analogous to the MIC, as it is based on bacterial growth quantification (Macia, Rojo-Molinero et al. 2014). For instance, by measuring changes to samples O.D, planktonic and dispersed 
cells always exist in the solution; lower turbidity can thus be a proxy to lower biofilm growth.

One of the first protocols describing MBIC testing with CBD has already clearly shown that these inhibitory values were much higher than conventional MIC values, and continued to increase as biofilms were allowed to grow for longer durations (Moskowitz, Foster et al. 2004). The same work has revealed that $P$. aeruginosa isolates from CF patients were displaying varying degrees of resistance, at most cases rendering antibiotic administration based on MIC inefficient in chronic infections.

An important parameter that can be determined is the minimal biofilm eradication concentration (MBEC), which is the concentration that results in eradication of $99 \%$ of biofilm bacteria. As for the MBC testing above, the REMA assay can be utilized to assess the MBEC (Dalecki, Crawford et al. 2016). Conversely, the synergy of two antimicrobials can be evaluated by a checkerboard antimicrobial challenge, as in FIC, by a fractional biofilm eradication concentration (FBEC) if a combination yields lower MBEC values than monotherapy (Figure 1.8). 


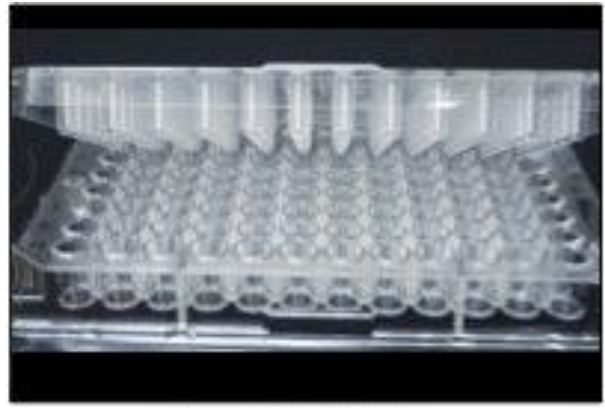

Growth Plate



Challenge Plate Checkerboard

Incubate Overnight



MBIC Measurement

Wash, Move Lid

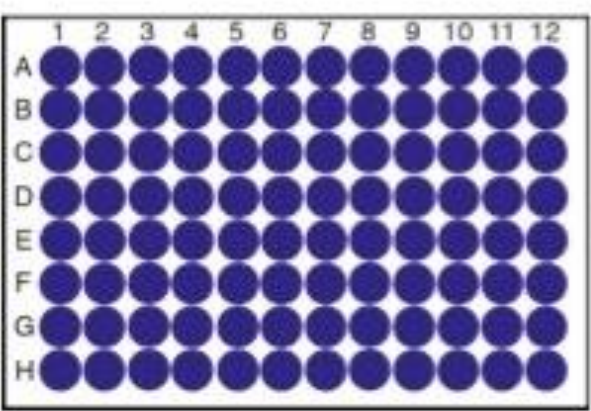

REMA Plate

Figure 1.8 The use of CBD to exclusively measure susceptibility of the biofilm fraction of bacteria. The challenge plate depicted here is organized as a checkerboard assay, with criss-cross gradients of two drugs, horizontally and vertically. MBIC is measured as turbidity, and the REMA assay is utilized to measure MBEC. 
Despite the inherent advantages of the CBD, it is not suitable for CFU measurements. The suggested protocols (Ceri, Olson et al. 1999, Moskowitz, Foster et al. 2004) involve mechanical disruption of biofilms to detach bacteria and constituted in this research a high variance in preliminary experimental results. To overcome this problem, a novel method exists that utilizes biofilms grown on $5 \mathrm{~mm}$ glass beads (Konrat, Schwebke et al. 2016). Beads-grown biofilms show a high level of repeatability, essentially the same techniques can be applied to bead biofilms, but beads can be more readily collected and handled.

The bead biofilm system allows performing time-dependent killing assays, necessary for understanding the efficacy of antimicrobial agents (Schwalbe, Steele-Moore et al. 2007). All discussed parameters above provide snapshot assessments of susceptibility, conventionally after 24-hour exposure, simulating a once-daily antibiotic regimen. Time-kill curves, of CFU values versus exposure time, allows calculating the minimal duration of killing (MDK), which is an important parameter when determining efficacy against slowgrowing bacteria, such as biofilm bacteria (Brauner, Fridman et al. 2016). $\mathrm{MDK}_{99}$ is the time point by which $99 \%$ of the population is eradicated, i.e. $2 \log _{10}$ reduction in CFU. By the same convention, a timed series of CFU determination during the course of 24 hours provides an understanding of antimicrobial killing kinetics.

The underlying problem for prediction of treatments to efficiently eradicate biofilms, apart from lack of comparative standards, is the duration of treatments (Malone, Goeres et al. 2017). High efficacy is dependent both on 
high concentrations of antibiotics and on long time scales, which in turn are dependent on diffusion through the matrix and different metabolic rates. Even when drug combinations are utilized, concentrations remain high and involve risks for toxicity in patients as well as deterioration of the quality of life. The most successful treatments still have no assurance that biofilms will not prevail somewhere, remaining a source of possible acute re-infection by dispersed cells (Bjarnsholt, Alhede et al. 2013). When evaluating new strategies to combat biofilms, the primary considerations are shortening treatment times (lower $\mathrm{MDK}_{99}$ ) to reduce the risk of emergence of antimicrobial resistance over prolonged exposure; and reducing relevant antibiotic concentrations required to eradicate biofilms to increase treatment efficacy (faster and greater killing effect).

\subsection{Research Gaps}

The development of biofilm-associated infections is one of the leading mechanisms that represent a niche for AMR to spread. Biofilms represent a diffusion barrier to antimicrobials, which protect bacteria. Standardized techniques used to study susceptibility of bacteria to antimicrobials focus predominantly on planktonic bacteria (Fournier, Drancourt et al. 2013), despite the suggestion that the majority of infections, especially chronic infections, are associated with biofilms. One strategy to better treat biofilm infections would be to initiate dispersal, where the dispersed cells are more susceptible to antibiotics (Kostakioti, Hadjifrangiskou et al. 2013, Yu, Su et al. 2015, Ren, Wu et al. 2016). 
The challenge of investigating the effectiveness of biofilm dispersal as part of a treatment strategy is that dispersal agents have yet been used extensively in a clinical setting (Fleming and Rumbaugh 2017). Furthermore, the use of antibiotic combinations in tandem with biofilm dispersal has never been applied clinically. The combined efficacy has the potential to allow for high synergy at in-vivo achievable antimicrobial concentrations, suggesting that the antimicrobial effect will occur in in-vivo conditions, during patients' therapy.

\subsection{Aims and Scope}

Using a strain of $P$. aeruginosa that can be induced to disperse as a model organism, this study aimed to provide a proof of concept of the therapeutic potential of biofilm dispersal. The study has three distinct but interrelated chapters that aimed at:

Chapter 2: Evaluating biofilm dispersal as a possible adjunct therapy. A $P$. aeruginosa strain containing an arabinose-inducible PDE enzyme was used to demonstrate that c-di-GMP mediated biofilm dispersal has the potential to increase antimicrobial synergy, by enhancing killing efficiency and lowering drug concentrations required for biofilm eradication.

Chapter 3: Assessing the risk of development of resistant variants due to exposure to antibiotic stress. Morphotype variants were compared to the parental strain for motility, biofilm production and MIC parameters. The effect of continuous induction of c-di-GMP mediated biofilm dispersal on antibiotic therapy was further evaluated. 
Chapter 4: Demonstrating the potential of c-di-GMP mediated biofilm dispersal as last resort strategy for treating bacterial biofilms of AMR strains. A colistin susceptible and a resistant isogenic pair of $\mathrm{PAO} 1 / \mathrm{p}_{B A D^{-}} y$ hjH strains were generated and fully characterized in order to investigate the potential for c-di-GMP mediated biofilm dispersal to eradicate biofilms of AMR strains. 


\section{Chapter 2: Evaluation of c-di-GMP Mediated Biofilm Dispersal as a Strategy to Restore Antibiotic Efficacy}

Based on the publication "In-vitro evaluation of biofilm dispersal as a therapeutic strategy to restore antimicrobial efficacy" (Roizman, Vidaillac et al. 2017).

\subsection{Introduction}

The current strategy to eradicate chronic bacterial biofilm infections involves long-term combinatorial antibiotic treatments at high dosages and the surgical removal of the infected tissue/foreign body (Høiby, Bjarnsholt et al. 2014). A notable example are treatments administered to CF patients, with chronic $P$. aeruginosa infections; combinations of broad-spectrum antibiotics (such as carbapenems) and specific anti-pseudomonal antibiotics (such as tobramycin) are administered to combat phases of acute infection and as a preventative measure against resistance development (Foweraker, Laughton et al. 2009). It is additionally known that these combinations have the potential for synergy, but experiments with clinical isolates show varied results. Prolonged antibiotic stress has been shown to eventually facilitate MDR, as exhibited in P. aeruginosa isolates from CF patients (Jansen, Mahrt et al. 2016).

Recently, there has been a growing interest in combining chemical agents that interfere with bacterial communication or signaling pathways with broadspectrum antibiotics to treat biofilm-associated infections (Jakobsen, TolkerNielsen et al. 2017). Thus, through attenuation of biofilm formation, these 
quorum sensing inhibitors restore the killing efficacy of antimicrobial treatments (Kostakioti, Hadjifrangiskou et al. 2013). C-di-GMP is a secondary messenger with multiple regulatory functions, including a central role in controlling biofilm formation. Higher intracellular levels of c-di-GMP enhance biofilm formation, while lower levels lead to biofilm dispersal with the potential to, at least partially, restore antimicrobial susceptibility. It has been recently shown that NO mediated c-di-GMP degradation leads to higher killing potential by tobramycin, of MDR clinical $P$. aeruginosa isolates from CF patients (Howlin, Cathie et al. 2017).

Even though all of the above phenomena are recognized, i.e. antibiotic combinations are preferred at treating chronic biofilm-related infections, and, c-di-GMP mediated dispersal potentiates the efficacy of specific antibiotics. There is a lack of proof-of-concept studies combining dispersal and combined antibiotic therapy. Due to the lack of clinical laboratory standards to test antibiotic synergy against biofilm models, in-vitro systems should first be optimized to investigate mature samples (Malone, Goeres et al. 2017). If a combinatorial treatment can lower the necessary drug concentrations and treatment durations, to efficiently eradicate biofilms - it can result in a considerable improvement of patient life quality. The present study tested the hypothesis that c-di-GMP mediated biofilm dispersal has the potential to enhance antimicrobial synergy and ultimately improve antimicrobial killing efficacy when applied to mature biofilms. 


\subsection{Methods}

Bacterial strains: Strains of $P$. aeruginosa used here consisted of PAO1/pJN105 a vector control carrying the araBAD promoter inducible by arabinose, and gentamicin resistance for selection $\left(\mathrm{Gm}^{r}\right)$. PAO1/p $\mathrm{p}_{B A D^{-}} y$ hjH carrying a plasmid containing $y h j H$ under the araBAD promoter. The YhjH PDE from Escherichia coli (E. coli) that degrades intracellular c-di-GMP. It has been transformed into the w.t strain of $P$. aeruginosa PAO1 and a protocol for the utilization of this system to study the effect of c-di-GMP mediated dispersal on PAO1 biofilm has been described previously (Chua, Hultqvist et al. 2015), and PAO1 w.t.

Chemicals: Lysogeny Broth Agar, Miller recipe (LB Agar, BD Difco) and Mueller-Hinton broth II or agar (MHB or MHA, Sigma-Aldrich) were used. Antimicrobial agents include tobramycin, imipenem, colistin, ciprofloxacin (Sigma-Aldrich), gentamycin (MP Biomedicals) and azithromycin (Tee Hai Chem).

Biofilm formation and induction of dispersal: Strains were streaked fresh from frozen stocks onto LB agar supplemented with gentamicin sulfate at $60 \mu \mathrm{g} / \mathrm{mL}$. Single colonies were picked into $2 \mathrm{~mL} \mathrm{MHB}$, in $10 \mathrm{~mL}$ falcon tubes, strains containing $\mathrm{Gm}^{r}$ were grown with supplemented $20 \mu \mathrm{g} / \mathrm{mL}$ gentamicin, w.t without. Inoculums were grown overnight (O.N.) at $37^{\circ} \mathrm{C}$ with shaking at $200 \mathrm{rpm}$, following which were centrifuged $6 \mathrm{~K}$ rpm 5 minutes. Pellets resolubilized in $6 \mathrm{~mL} \mathrm{MHB}$, and their turbidity was measured as O.D at $600 \mathrm{~nm}$ with a UV-Vis Spectrophotometer (Shimazu). Serial dilutions in MHB were initially used for colony forming units $(C F U / m L)$ enumeration versus $O . D_{600}$. 
Inoculums at 0.005 O. $D_{600}$ final turbidity, concurring to $\sim 10^{6} \mathrm{CFU} / \mathrm{mL}$, as advised by CLSI (CLSI 2012, Malone, Goeres et al. 2017), for an initial inoculum size of $\sim 5 \times 10^{5}-10^{6} \mathrm{CFU} / \mathrm{mL}$ and is used in all further experiments.

Different static, in-vitro systems were used to grow biofilms, 24-well and 96-well microplates (NUNC ${ }^{\mathrm{TM}}$, Thermo-Fischer) were used in high-throughput with Infinite Pro 200 microplate reader (Tecan). Confocal experiments (CLSM) with biofilms were conducted using ibidi $^{\circledR}$ eight-well $(200 \mu \mathrm{L})$ glass bottom $\mu$-slides. Where indicated the Calgary Biofilm Pin Lid Device (CBD) (NUNC ${ }^{\mathrm{TM}}$, Thermo-Fischer) (Ceri, Olson et al. 1999) 96-well peg lid system or $5 \mathrm{~mm}$ glass beads (Merck) were used as substratum for biofilm growth. Biofilms were grown in each system using MHB unless indicated otherwise, at a $37^{\circ} \mathrm{C}$ incubator, without shaking. For experiments with duration of over 1-day a washing and media replenishing step were applied daily: wells were washed with $0.9 \% \mathrm{NaCl}$ (in the case of $\mathrm{CBD}$ a wash plate was used) and replenished with fresh MHB, samples were maximally grown for 3 days. If CBD was used the peg lid was transferred daily into a clean 96-well plate containing $200 \mu \mathrm{L}$ $0.9 \% \mathrm{NaCl}$ in each well to rinse off loosely attached cells and then transferred to a plate with fresh MHB medium.

Arabinose-induced dispersal: To induce c-di-GMP mediated dispersal and test its effects on the model PAO1/p $\mathrm{p}_{\mathrm{BAD}}-y h j H$ biofilms, samples were exposed following wash with $0.9 \% \mathrm{NaCl}$, to fresh $\mathrm{MHB}$ containing $0.25-1 \%$ weight per volume (w/v) arabinose for 4 hours (Chua, Tan et al. 2013). Control experiments were performed in parallel with $\mathrm{PAO} 1 / \mathrm{p}_{\mathrm{BAD}}-y$ hjH using an arabinose-free medium, and with the control strains. The effects of c-di-GMP 
mediated dispersal were validated as a change in sample turbidity at $O . D_{600}$ and $\mathrm{CFU} / \mathrm{mL}$ measurement of the resulting sample medium.

Biofilm production quantification: Crystal violet (CV) staining was performed with a stock solution of $0.01 \%$ volume per volume (vol/vol) CV (Sigma-Aldrich) in $\mathrm{dH}_{2} \mathrm{O}$ (MilliQ) (O'Toole 2011, Chua, Hultqvist et al. 2015). 24-well plates were used to grow 1-day biofilms, liquid from O.N. biofilm growth was carefully pipetted out (not disturbing the biofilm at the bottom and on sides of wells) and washed with $0.9 \% \mathrm{NaCl} .1 \mathrm{~mL}$ of $\mathrm{CV}$ solution was added, and the plate left at room temperature with shaking at $80 \mathrm{rpm}$, for 30 minutes. The excess stain was then removed by pipetting and the plates immersed in water, decanted and left to dry upside down on the bench. The stain was then solubilized with $1 \mathrm{~mL}$ ethanol in each well and plates left on a shaker at $80 \mathrm{rpm}$ for 15 minutes. CV Absorbance (Abs) at $590 \mathrm{~nm}$ was measured using an Infinite Pro 200 microplate reader (Tecan).

Inhibitory antibiotic concentrations: Minimum inhibitory concentrations (MIC) were assessed by manual un-aided inspection of sample growth followed by turbidity $\mathrm{O} . \mathrm{D}_{600}$ measurement in a microplate reader. Experiments with planktonic samples had $5 \times 10^{5}-10^{6} \mathrm{CFU} / \mathrm{mL}$ in each well, as advised by CLSI (CLSI 2012). MIC was determined by broth microdilution, using two-fold dilution gradients of different antimicrobials. Following 24 hour incubation at $37^{\circ} \mathrm{C}$, sample turbidity was measured as O. $\mathrm{D}_{600}$. Values higher than 3 times the blank controls wells were considered as indicative of growth, thus below the MIC range. Multiple experimental replicates were performed and included three independent biological replicates for each condition tested as well as two 
technical replicates. Experimental results are expressed as the average values +/- standard deviation (STDEV) with $\mathrm{N}=6$.

Minimal bactericidal concentration (MBC): To determine the MBC values directly over-and-above of MIC values, two techniques were employed. First, $10 \mu \mathrm{L}$ aliquots were sampled from each well in each concentration above the MIC. Samples were drop plated on LB agar and incubated O.N. at $37^{\circ} \mathrm{C}$, and at the following day (after 20-24 hours) visually inspected for growth. MBC was determined as the antibiotic concentration in wells that did not contain any viable bacteria as could be detected.

Second, MBC was evaluated by the resazurin microtiter assay (REMA) (Sandberg, Schellmann et al. 2009). $10 \mu \mathrm{L}$ of resazurin stock solution, prepared as $0.3 \mathrm{mg} / \mathrm{mL}(\sim 1.2 \mathrm{mM})$ were added to each well to a final concentration of $15 \mu \mathrm{g} / \mathrm{mL}(60 \mu \mathrm{M})$. Plates were incubated for $3-4$ hours at $37^{\circ} \mathrm{C}$, and fluorescence was measured in an Infinite Pro 200 microplate reader (Tecan) with excitation at $550 \mathrm{~nm}$ and emission at $590 \mathrm{~nm}$ under automatic optimal gain. Wells' fluorescence values equal or higher than 3 times the fluorescence value of the control blank wells were considered viable, and thus, under the $\mathrm{MBC}$ range.

Antimicrobial synergistic assays against biofilms: The synergistic potential of tobramycin and imipenem, as well as the impact of induced biofilm dispersal, were assessed in a checkerboard manner (Jenkins and Schuetz 2012). "Challenge plates" consisting of two-fold gradients of both tobramycin $(0.25$ to $64 \mu \mathrm{g} / \mathrm{mL}$ ) and imipenem (1 to $2048 \mu \mathrm{g} / \mathrm{mL}$ ) in MHB were prepared. Plates were prepared so that drug A was diluted in rows (horizontally) and drug B 
diluted in the columns, with column 12 reserved for controls (growth and negative), column 1 and row A were monotherapy (Dundar and Otkun 2010).

Monotherapy minimal biofilm eradication concentrations (MBEC) values and fractional biofilm eradication concentration (FBEC) indices for combinations were determined for 2-3 day grown CBD biofilms in MHB. Biofilms were formed by inoculating $P$. aeruginosa into the wells of a 96 -well plate at a final density of $5 \times 10^{5}-1 \times 10^{6} \mathrm{CFU} / \mathrm{mL}$ and incubation at $37^{\circ} \mathrm{C}$. CBD biofilms were grown as described above for 3 days, following which induced dispersal was applied for 4 hours as described, or not. Biofilms were then transferred to the Challenge plate and incubated for 24 hours at $37^{\circ} \mathrm{C}$. Following challenge, biofilms were washed in plates with $0.9 \% \mathrm{NaCl}$ and subsequently transferred to a plate with $200 \mu \mathrm{L} \mathrm{MHB}$ media with $60 \mu \mathrm{M}$ resazurin for 4 hours at $37^{\circ} \mathrm{C}$, to determine the viability of the biofilm bacteria. Viability was measured by fluorescence quantification in a microplate reader, as described above for MBC. For monotherapy, MBEC values were defined as the lowest drug concentrations resulting in fluorescence values that were no more than twice the negative controls. FBEC and $\Sigma F B E C$ values were calculated as previously described (Shafiei, Abdi Ali et al. 2014): A value of 1 was given to the MBEC of mono-treatment, and following the two-fold dilution manner, a value of $0.5^{\mathrm{N}}$ ( $\mathrm{N}$ being the number of dilutions) was given to the corresponding wells in the combined treatment. Values for both drugs were summed to $\Sigma F B E C$, combinations with a $\Sigma F B E C$ value $\leq 0.5$ were considered synergistic.

Biofilm time-kill kinetics to assess $\mathrm{MDK}_{\underline{9 g}}$ : Antibiotic susceptibility was determined as a factor of time. Here, biofilms were grown for 3 days using 
$5 \mathrm{~mm}$ glass beads (Konrat, Schwebke et al. 2016) in MHB. Two beads (representing two technical replicates, verifying reproducibility) were placed into each well of a 24-well microtiter plate and exposed to fresh $\mathrm{MHB}$ containing $1 \%(\mathrm{w} / \mathrm{v})$ arabinose or arabinose free medium (control samples). After 4 hours, samples were treated with imipenem and tobramycin $(8 \mu \mathrm{g} / \mathrm{mL})$ alone or in combination. Biofilms were removed from the beads at different time points by alternating 10 -second sonication in a water bath (at $37 \mathrm{kHz}$ ) and 10 seconds of vortexing (both at $100 \%$ power). Bacterial suspensions were subsequently serially diluted and drop-plated onto LB agar plates. After 24 hours incubation at $37^{\circ} \mathrm{C}$, the colony forming units were determined as average of CFU/bead and plotted against time. Experiments were performed in five independent biological replicates to ensure reproducibility; results are expressed as the average value +/- STDEV, $(\mathrm{N}=5)$.

Confocal Microscopy: The efficacy of the combined antibiotic therapy was also examined using confocal laser scanning microscopy (CLSM, Zeiss 780, Germany). Biofilm samples were grown on eight-well $(200 \mu \mathrm{L})$ glass bottom $\mu$-slides for 3 days before exposure to imipenem and tobramycin (at $8 \mu \mathrm{g} / \mathrm{mL}$ ) alone or in combination. After the 24 hours antibiotic challenge, samples were stained for 10 minutes, using the LIVE (Syto-9)/DEAD (Propidium lodide, PI) BacLight $^{\mathrm{TM}}$ Bacterial Viability $\mathrm{Kit}^{\circledR}$. Syto-9 and PI fluorescence measurements were conducted by excitation with 488 and $561 \mathrm{~nm}$ laser lines, respectively. Four images (considered as technical replicates) were acquired in Z-stacks for each sample. The ratio of average bio-volumes $\left(\mu \mathrm{m}^{3} / \mu \mathrm{m}^{2}\right)$ calculated for live and dead stained populations in each image was quantified using COMSTAT 2 ImageJ plugin (Heydorn, Nielsen et al. 2000). A constant threshold of 50 
was set for each channel. Experiments were performed in five independent biological replicates to ensure reproducibility; results are expressed as the average +/- STDEV $(\mathrm{N}=5)$.

Statistical analysis: Statistical significance analysis (using multiple student's t-test or two-way ANOVA, where indicated) using software recommended method for each experiment and final graph plotting were all conducted using Prism GraphPad 7.

\subsection{Results}

\section{$\underline{\text { Antimicrobial susceptibility testing against planktonic cultures }}$}

PAO1 strains Inocula at O. $\mathrm{D}_{600} 0.01$ were preliminarily found to correlate to $1.5 \times 10^{6} \mathrm{CFU} / \mathrm{mL}$ (Figure 2.1C) yielding a final inoculum density of $5 \times 10^{5}-10^{6}$ CFU/mL in experimental wells for all experiments, as instructed by CLSI (CLSI 2012). To determine the optimal medium for subsequent c-di-GMP mediated dispersal assays, biofilms were formed for 24 hours in four different media, ABTGC (Chua, Hultqvist et al. 2015), MHB, TSB (Sigma-Aldrich) and LB and the dispersal response of these biofilms was determined. Dispersal was induced by adding arabinose, which controls the expression of the phosphodiesterase $y$ hjH and dispersal in the presence and absence of arabinose after 4 hours of incubation was also compared.

The amount of biofilm formed, as determined by CV staining, was similar for ABTGC, TSB and LB (Abs ${ }_{590}$ between 0.125 and 0.15 ). In contrast, there was significantly more biofilm formed in MHB $(0.21+/-0.002)$ compared to the 
other media (Figure 2.1A). While arabinose was able to induce dispersal of biofilms grown in all media, dispersal was only significant for MHB grown biofilms, where approximately $55 \%$ of the biofilm was dispersed after arabinose addition $\left(\mathrm{Abs}_{590} 0.11+/-0.001\right)$. It is unknown why dispersal had a stronger effect in MHB media or why such difference occurs in CV stain as compared to other media. It was suggested in other works that the higher $\mathrm{Ca}^{+2}$ concentrations might be the main effector for PAO1 (Jain, Parida et al. 2013). In general, all other media did not show any significant difference in CV stain with or without arabinose addition (Average values between 0.09-0.14). Dispersal was also quantified by measuring the $O . D_{600}$ of the planktonic cells (Figure 2.1B), which increased $47 \%$ to $66 \%$ for the different media used, relative to the control without arabinose induction. Similarly, the CFU counts were higher in the planktonic phase after arabinose addition, where the CFUs increased $140 \%(+/-3.35 \%)$ for ABTGC-grown cells and $232 \%(+/-0.28 \%)$ for MHB biofilms. MHB showed the most significant difference between treated and untreated samples (t-test, $\mathrm{p}$-value $<0.001$ ). Based on these results, subsequent experiments used MHB medium since this medium showed the highest amount of total biofilm formed $\left(\mathrm{Abs}_{590}\right.$ of $\left.0.22+/-0.003\right)$ and the highest amount of dispersal (55\%). Further, MHB is the recommended medium for MIC testing based on the standardized protocol (CLSI 2012). 
A Biofilm production in different media

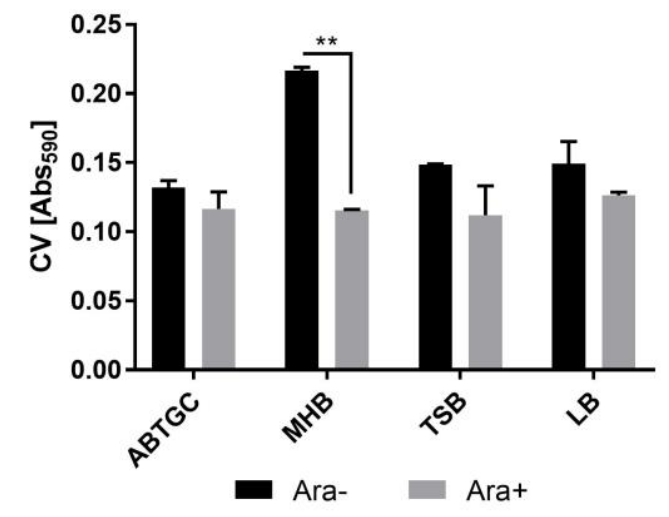

C



B



D

D $\quad$ CFU/mL of dispersed cells

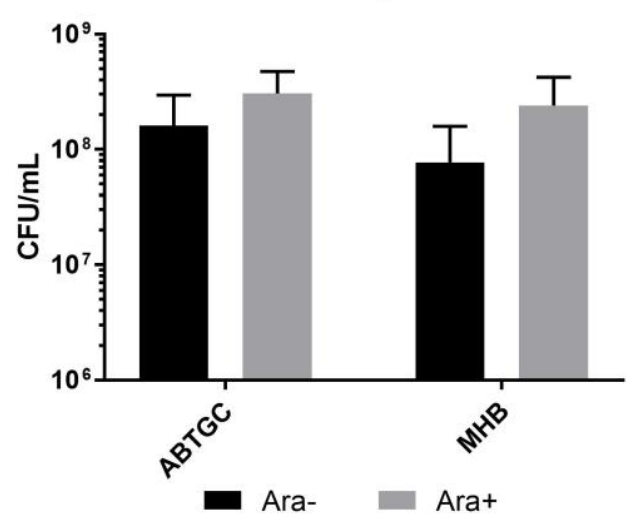

Figure 2.1 The effect of medium on the c-di-GMP mediated dispersal response of Pseudomonas aeruginsoa PAO1/p $\mathrm{p}_{B A D^{-}}$hhjH biofilms, carrying the arabinose-inducible phosphodiesterase $y$ hiH, were formed in ABTGC, MHB, TSB and LB for quantification of biofilm formation and dispersal. The amount of biofilm formed (A) and dispersed upon arabinose addition as measured by CV staining ( Abs $_{590}$ ), resulting changes to sample turbidity $\left(O . D_{600}\right)$ (B) following induced dispersal. Establishment of initial inocula conditions for all further experiments (C) as turbidity vs. $\mathrm{CFU} / \mathrm{mL}$ and a comparison of induced dispersal as CFU $/ \mathrm{mL}$ (D) of the supernatant above the biofilms for treated and untreated samples in ABTGC and MHB media. Values represent the average of 3 independent experiments, +/STDEV; ${ }^{* *}$ : p-value $<0.001$ multiple t-test, using the Bonferroni-Dunn method, $\alpha=0.05$.

To ensure that the plasmid vectors used did not affect the drug susceptibility profile, imipenem MICs and MBCs values of $P$. aeruginosa PAO1 were compared to $P$. aeruginosa / $\mathrm{p}_{B A D}-y$ hjH and $P$. aeruginosa / pJN105 (Figure 2.2 
A and B). On the basis of the O.D 600 measurements, no difference in MIC values was observed (Figure 2.2A). All three strains were completely inhibited at $1 \mu \mathrm{g} / \mathrm{mL}$ and showed approximately a $50 \%$ growth reduction at $0.5 \mu \mathrm{g} / \mathrm{mL}$. In contrast, there was no significant difference in the MBC for the three strains as measured using the REMA assay, which monitors respiratory activity (Figure 2.2B). The MBC of imipenem for these strains was $1-2 \mu \mathrm{g} / \mathrm{mL}$.

Subsequently, the MIC and MBC values were determined for imipenem and colistin (Figure 2.2 B and C) as well as tobramycin and azithromycin (Figure 2.2 D and E) specifically against $P$. aeruginosa $/ \mathrm{p}_{B A D}-y h j H$. Both imipenem and colistin showed identical MIC and MBC patterns, where $1 \mu \mathrm{g} / \mathrm{mL}$ of both compounds completely inhibited growth or activity of $P$. aeruginosa. Tobramycin was inhibitory between $0.5-1 \mu \mathrm{g} / \mathrm{mL}$ while azithromycin showed a MIC value of 4-8 $\mu \mathrm{g} / \mathrm{mL}$. Tobramycin and azithromycin differed in MBCs, where $4 \mu \mathrm{g} / \mathrm{mL}$ of tobramycin was bactericidal compared to $8 \mu \mathrm{g} / \mathrm{mL}$ for azithromycin. Thus, the difference in activity between these two antibiotics was more apparent in these assays, where tobramycin showed a 4-fold increase between MIC and MBC but azithromycin showed a maximum 1-fold increase in those values. The values observed here, for both the MICs and MBCs are consistent with published results (Table 2.1). 



$$
\text { pJN105 } \leftarrow \text { w.t. }
$$
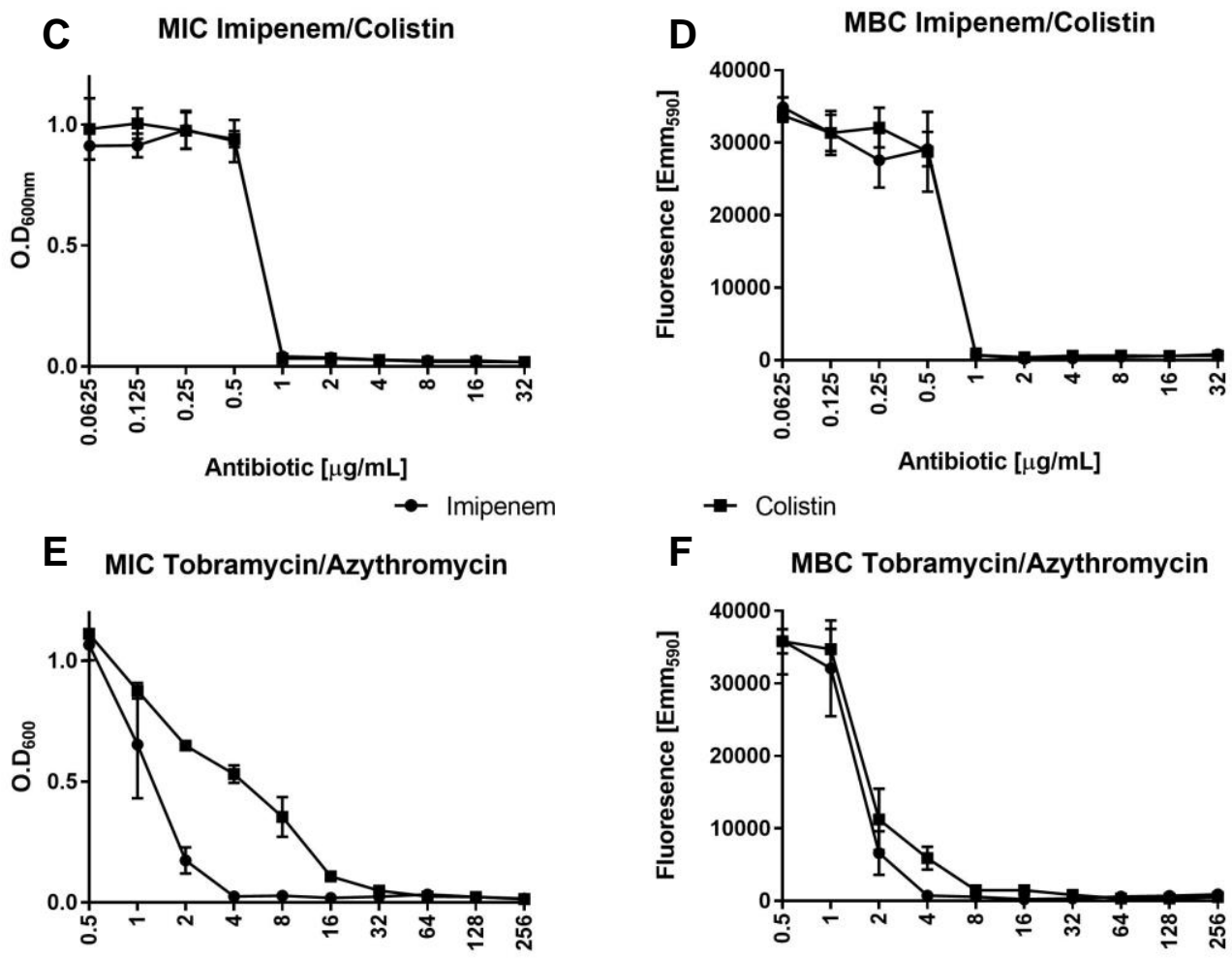

Antibiotic $[\mu \mathrm{g} / \mathrm{mL}]$
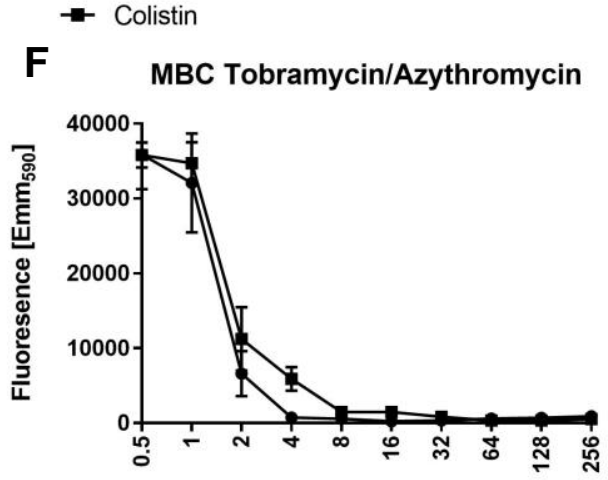

Antibiotic $[\mu \mathrm{g} / \mathrm{mL}]$

Figure 2.2 MICs $\left(O . D_{600}\right)$ and MBCs as measured using the REMA assay (emission at $590 \mathrm{~nm}$ ) for four antibiotics. MICs (A, C and E) and MBCs (B, D and F) were determined for imipenem against the w.t $P$. aeruginosa PAO1 strain, $\mathrm{PAO} 1 / \mathrm{p}_{B A D^{-}} y$ hjH and PAO1/pJN105 carrying the empty vector (A and B), while the MIC and MBCs were determined for imipenem \& colistin (C and $\mathbf{D})$ or tobramycin \& azithromycin (E and $\mathbf{F})$ for $\mathrm{PAO} 1 / \mathrm{p}_{B A D}-y$ hjH. All measurements were made following 24-hour exposure at $37^{\circ} \mathrm{C}$. All experiments were performed in triplicate, and the error bars are $+/-$ STDEV. Antibiotic concentrations are shown on a $\log _{2}$ scale (thus the control with $0 \mu \mathrm{g} / \mathrm{mL}$ concentration could not be plotted). 
Because the REMA method is not a commonly used standard for defining MBCs, the results obtained by this method were subsequently compared with MBC results by the drop-plate method (Table 2.1). Visual inspection and O. $\mathrm{D}_{600}$ counts gave comparable MICs for all four antibiotics. These were similar to published values or were different by one dilution (for example, $0.5-1 \mu \mathrm{g} / \mathrm{mL}$ compared to $1-2 \mu \mathrm{g} / \mathrm{mL}$ for imipenem). The MBC as determined by drop plating at most 2 -fold higher than the value obtained using the REMA assay for all antibiotics tested. REMA was found to be suitable for consecutive high-throughput microplate-based assays.

Table 2.1 Evaluation of MIC and MBC of 4 different antibiotics for PAO1/p $\mathrm{p}_{B A D}-y h j H$ by different assays and comparison to literature results.

\begin{tabular}{|l|c|c|c|c|c|c|l|}
\hline Antibiotic & \multicolumn{3}{|c|}{ MIC } & \multicolumn{3}{c|}{ MBC } & References \\
\hline & $\begin{array}{l}\text { Visual } \\
\text { reading } \\
{[\mu \mathrm{g} / \mathrm{mL}]}\end{array}$ & $\begin{array}{l}\text { Reader } \\
\mathrm{OD}_{600} \\
{[\mu \mathrm{g} / \mathrm{mL}]}\end{array}$ & $\begin{array}{l}\text { Literature } \\
{[\mu \mathrm{g} / \mathrm{mL}]}\end{array}$ & $\begin{array}{l}\text { Drop } \\
\text { plate } \\
{[\mu \mathrm{g} / \mathrm{mL}]}\end{array}$ & $\begin{array}{l}\text { REMA } \\
\mathrm{Emm}_{600} \\
{[\mu \mathrm{g} / \mathrm{mL}]}\end{array}$ & $\begin{array}{l}\text { Literature } \\
{[\mu \mathrm{g} / \mathrm{mL}]}\end{array}$ & \\
\hline Imipenem & $0.5-1$ & $0.5-1$ & $1-2$ & $2-4$ & $0.5-1$ & $2-4$ & $\begin{array}{l}\text { Riera et al (2011) } \\
\text { Meletis et al. (2012) }\end{array}$ \\
\hline Colistin & $0.5-1$ & $0.5-1$ & $1-2$ & $2-4$ & $0.5-1$ & $2-4$ & $\begin{array}{l}\text { Hermann, et al. (2010) } \\
\text { Hengzhuang et al. (2012) }\end{array}$ \\
\hline Tobramycin & $1-2$ & $2-4$ & $2-4$ & $2-4$ & $2-4$ & $2-4$ & $\begin{array}{l}\text { Mah et al. (2003) } \\
\text { Tré-Hardy et al. (2010) }\end{array}$ \\
\hline Azythromycin & $16-32$ & $16-32$ & $16-32$ & $32-64$ & $16-32$ & $32-64$ & $\begin{array}{l}\text { Buyck et al. (2012) } \\
\text { Nalca et al. (2006) }\end{array}$ \\
\hline
\end{tabular}

Validation of the time of biofilm formation and the amount of arabinose to be used for induction of c-di-GMP mediated dispersal

Experiments were performed to verify arabinose mediated induction of c-diGMP mediated dispersal by the PAO1/p $\mathrm{p}_{B A D^{-}-y h j H}$ strain and that arabinose addition did not alter the dispersal response or biofilm development in the absence of the YhjH phosphodiesterase. For PAO1, 24-hour grown biofilms 
exhibited lower MBEC values than 48 and 72 hours (2-3-day grown biofilms) (Wu, Moser et al. 2015). It has also been shown that MBEC values increase by up to 1000 fold for 3-day biofilms ("mature") compared to earlier time points (Hengzhuang, Wu et al. 2011). Therefore, to determine if the time of biofilm development affected the antibiotic resistance profiles in the systems used here, biofilms were grown for 24, 48 and 72 hours and compared for antibiotic responses. Similarly, the concentration of arabinose used in the literature to induce the araC promoter varies from $0.25 \%$ and $1 \%$, and therefore, a range of arabinose concentrations was tested here to optimize induction of dispersal (Chua, Tan et al. 2013).

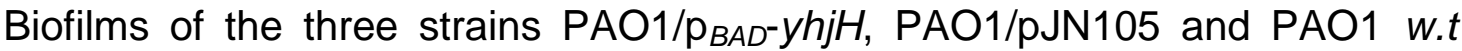
were grown in 24-well microplates for periods of 24,48 and 72 hours (1-3 days) with daily media replenishment as described. Samples were dispersed by arabinose addition and 4-hour incubation. Significant differences were evident by $\mathrm{CV}$ stain between $\mathrm{p}_{B A D^{-}}$yhjH and the two control strains $\left(\mathrm{Abs}_{590}\right.$ of 0.11 versus 0.14 ) only for biofilms grown for 72 hours after induction with $1 \%$ arabinose $(p<0.001)$, (Figure 2.3A). Generally the biofilms of all 3 strains became less varied after 48 and 72-hour growth and might indicate a change in the bacterial population in the samples, resulting in similar EPS production. Turbidity measurements of samples supernatant O.D $D_{600}$ showed no significant differences following dispersal with arabinose at any concentration. Arabinose exposure seemed to results in some level of dispersal from biofilms not related to any specific strain and might hint the involvement of a different mechanism. 
A

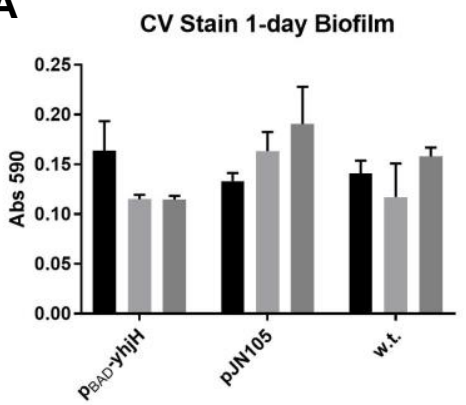

B

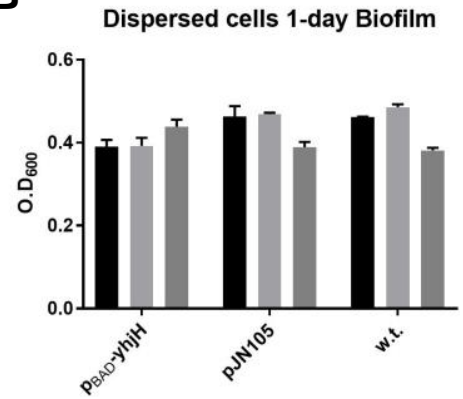

C

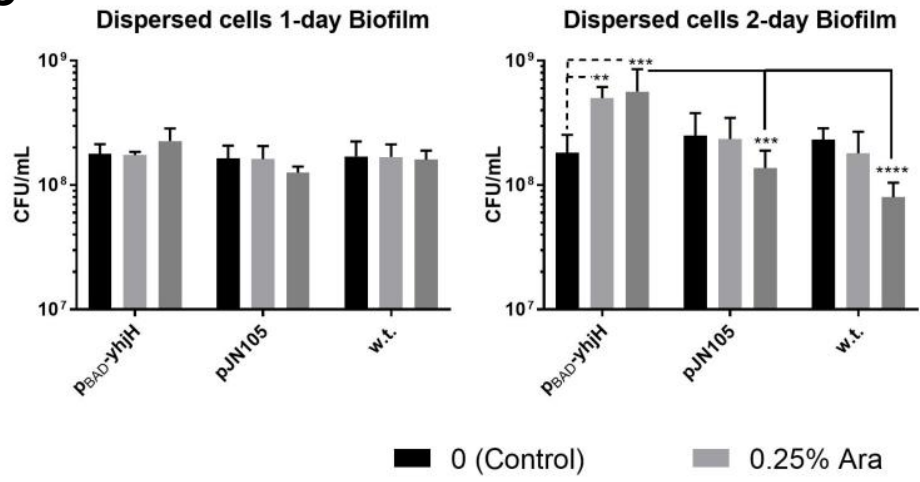

CV Stain 2-day Biofilm

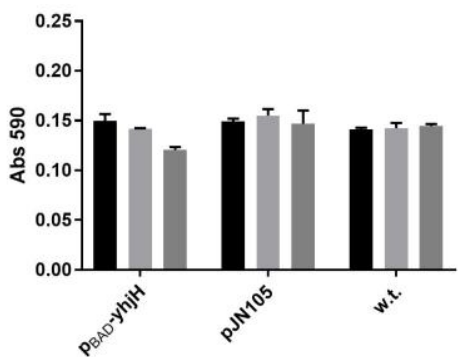

Dispersed cells 2-day Biofilm
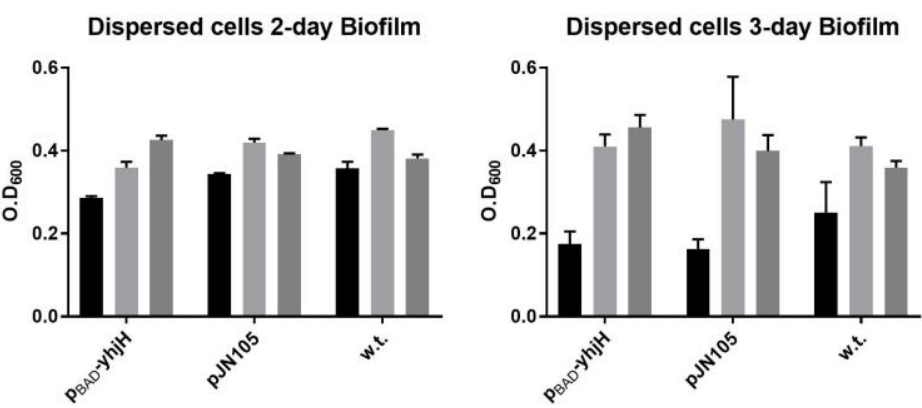

Dispersed cells 3-day Biofilm



$1 \%$ Ara

Figure 2.3 Effects of 4-hour arabinose (Ara) induction on 24, 48 and 72-hour grown biofilms of PAO1 strains $\mathrm{p}_{B A D}-y h j H$, pJN105, and w.t grown in 24-well plates. (A) Remaining biofilm stained by crystal violet (CV) measurement $\left(\mathrm{Abs}_{590}\right)$, (B) Turbidity O. $D_{600}$ and (C) CFU/mL of dispersed cells in the sample supernatant. Results were analyzed by two-way ANOVA. Statistically significant differences are given as corresponding p-value, interpretation: ${ }^{* *}<0.01^{* * *}<0.001^{* * * *}<0.0001$. Experiments were performed in triplicates $(\mathrm{N}=3)$ and plotted as average values +/- STDEV.

$\mathrm{CFU} / \mathrm{mL}$ measurements of samples supernatant indicate a significant trend following the 4 hours of arabinose-mediated dispersal (Figure 2.3C). For biofilms grown in this system, the growth stage that seems to exhibit the most 
profound differences between dispersed samples to controls are biofilms grown for 48 hours and more. For $\mathrm{p}_{B A D^{-}} y$ hjH arabinose induced 4-hour dispersal resulted in an increase in $\mathrm{CFU} / \mathrm{mL}$ of samples supernatant, from $1.8 \times 10^{8}$ in the control to $5 \times 10^{8}$ and $5.65 \times 10^{8}$ for $0.25 \%$ and $1 \%$ arabinose respectively more than $2.5-3$ times accordingly. $1 \%$ arabinose was more significant at this stage as indicated by two-way ANOVA $(\mathrm{p}<0.001)$ in relation

to the controls pJN105 and PAO1 w.t under the same conditions $\left(1.4 \times 10^{8}\right.$ and $0.7 \times 10^{8}$ respectively).

Biofilms of all strains grown for 72 hours prior to induced c-di-GMP mediated dispersal follow, in principle, the same trends indicated above, but the same treatment is more significant. $\mathrm{CFU} / \mathrm{mL}$ of the supernatant above the biofilm was $1.13 \times 10^{8}$ in the control to $4.20 \times 10^{8}$ and $5.23 \times 10^{8}$ respectively for $0.25 \%$ and $1 \%$ arabinose. Both were also more significant against both control strains, most with $1 \%$ arabinose against pJN105 and PAO1 w.t $\left(1.33 \times 10^{8}\right.$ and $1.58 \times 10^{8}$ respectively). The experimental results thus indicate, that induced c-di-GMP mediated dispersal with $1 \%$ arabinose for PAO1/p $\mathrm{P}_{B A D}-y$ hjH grown biofilms, resulted in 3 times higher $\mathrm{CFU} / \mathrm{mL}$ in the supernatant versus controls, presumably the dispersed cell fraction; results were more profound for 3-day grown biofilms.

\section{Antimicrobial susceptibility of biofilms}

3-day grown $P$. aeruginosa have been found to represent mature biofilms exhibiting 100-1000 fold higher antibiotic resistance (Herrmann, Yang et al. 2010, Hengzhuang, Wu et al. 2012). Preliminary experiments testing synergy between antimicrobial agents using REMA and CBD biofilms to establish 
MBEC where were conducted in a checkerboard manner, as described in the materials and methods section. The technique allowed determining the range of effective concentrations for monotherapy and a combinatorial gradient of both drugs, the MBEC and $\angle F B E C$ allowing comparison of fractional contribution of both drugs to synergy. Several combinations of antimicrobials were tested against 3-day grown biofilms of $\mathrm{PAO} 1 / \mathrm{p}_{B A D}-y h j H$ and PAO1/pJN105. The combination depicting the highest synergy in preliminary experiments (data not shown): tobramycin and imipenem - has been chosen to be the focus of all following proof-of-concept experiments.

Arabinose induction of c-di-GMP mediated biofilm dispersal had no impact on imipenem and tobramycin mono-treatment MBEC values $(2048$ and $64 \mu \mathrm{g} / \mathrm{mL}$ for imipenem and tobramycin, respectively) for both PAO1/pJN105 and PAO1/p $\mathrm{p}_{B A D^{-}} y$ hjH using this essay. Because tobramycin is the more efficient monotherapy, in this case, any change to its FBEC value reduces the combined $\Sigma$ FBEC value more prominently. Induction of c-di-GMP mediated dispersal significantly (One way ANOVA analysis, $p>0.1$ ) enhanced killing of PAO1/p $\mathrm{p}_{B A D}-y$ hjH upon dual treatment with imipenem and tobramycin compared to the same combination without arabinose induction of dispersal (Figure 2.4A). $\Sigma$ FBEC values were ranging from 0.012 to 0.017 versus 0.024 to 0.033 for non-dispersed biofilms. This effect was not observed in biofilms formed by the control strain PAO1/pJN105 (Figure 2.4B). 

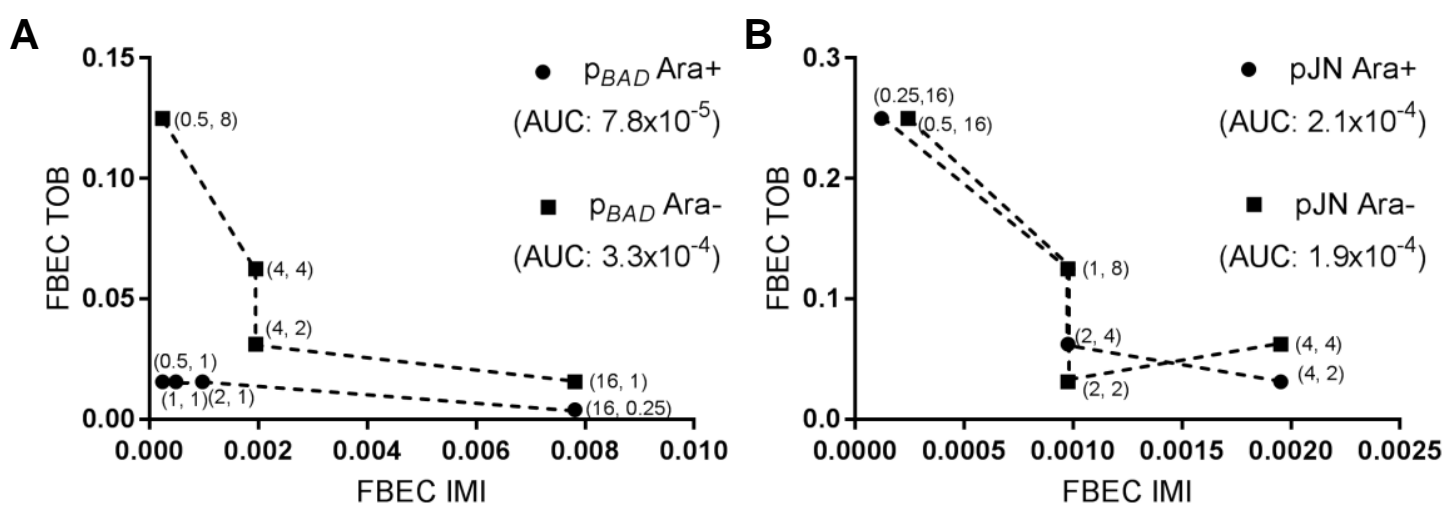

Figure 2.4 Isobolograms illustrating the relative fractional biofilm eradication concentration (FBEC) values for imipenem (IMI) and tobramycin (TOB). Absolute concentration values $[\mu \mathrm{g} / \mathrm{mL}]$ are indicated at points. Results are the lowest $\Sigma$ FBEC values from five independent experiments. Combined antibiotic 24-hour treatment against 3-day grown biofilms of strains PAO1/p BAD $^{-y h j H}(\mathbf{A})$ and PAO1/pJN105 (B) with (Ara+) or without (Ara-) 4 hours induced dispersal with $1 \%$ arabinose.

Imipenem and tobramycin MBEC values in combinations were thus significantly lowered as a combination (up to 7 and 3 -fold $\log _{2}$ decrease, respectively), after c-di-GMP mediated dispersal, compared to non-arabinose treated $\mathrm{PAO} 1 / \mathrm{p}_{B A D^{-}}$yhjH biofilms. Because this combination already exhibited a high synergy, i.e. $\sum$ FBEC values a magnitude lower than 0.5 , the area under the curve $(A \cup C)$ was calculated to better illustrate each isobologram (PenaMiller, Laehnemann et al. 2013). AUC for combined treatments against $\mathrm{p}_{B A D^{-}} y$ hjH was calculated to be $7.8 \times 10^{-5}[\mathrm{AU}]$ with induced dispersal versus $3.3 \times 10^{-4}[\mathrm{AU}]$ in the absence of dispersal, for the pJN105 no difference was evident.

The stronger synergistic effect following induced biofilm dispersal was further confirmed using time-kill assessments (Figure 2.5 A\&B). Biofilms of $p_{B A D^{-}} y h j H$ 
and pJN105 that were formed for 3 days on $5 \mathrm{~mm}$ glass beads in 24-well plates were used for this experiment. Beads were collected, and CFU/bead were determined for mono and combined imipenem and tobramycin treatments at the indicated time points. Both antibiotics were in the concentration of $8 \mu \mathrm{g} / \mathrm{mL}, 2$ times higher than the original MBC, $4 x \mathrm{MIC}$ and 1/8 of tobramycin's MBEC, concentrations not facilitating complete biofilm eradication, but allowing analysis of synergy. The time -4 hours indicates the addition of $1 \%$ arabinose, following 4 hours induction, samples were washed and treated as indicated. Plates were sampled at $-4,0,4,8,16$ and 24-hour time points. Synergy was defined as $2 \log _{10}$ reduction in CFU/Bead in the combined treatment versus the most potent antibiotic monotherapy, tobramycin alone.

The highest reduction in CFU/Bead was at the 24-hour time point, $\left(5.8 \log _{10}\right.$ $(\mathrm{CFU} / \mathrm{Bead})+/-0.34)$ for $\mathrm{p}_{B A D^{-}} y h j H$ following induced dispersal and combined antibiotic treatment. This value did not represent an obvious synergy between both drugs, versus tobramycin mono-treatment without induced dispersal at that time point $\left(7.36 \log _{10}(\mathrm{CFU} /\right.$ Bead $\left.)+/-0.31\right)$, a difference of $\sim 1.56 \log _{10}$. Still, this combination represents $99 \%$ killing, versus all control groups, (7.85$\left.7.77 \log _{10}(\mathrm{CFU} / \mathrm{Bead})+/-0.35\right)$, and $\mathrm{MDK}_{99}$ is in the range of $16-24$ hours. Lastly, lower CFU/Bead due to combined treatment could be evident after 8 hours exposure in all samples, independent of dispersal induction, but only the dispersed $\mathrm{p}_{B A D^{-}}$yhjH did not recover to $7-8 \log _{10}(\mathrm{CFU} / \mathrm{Bead})$ at the final time point, exhibiting sustained eradication. 
A

$\mathrm{p}_{B A D}-y h j \mathrm{H}$ Ara+

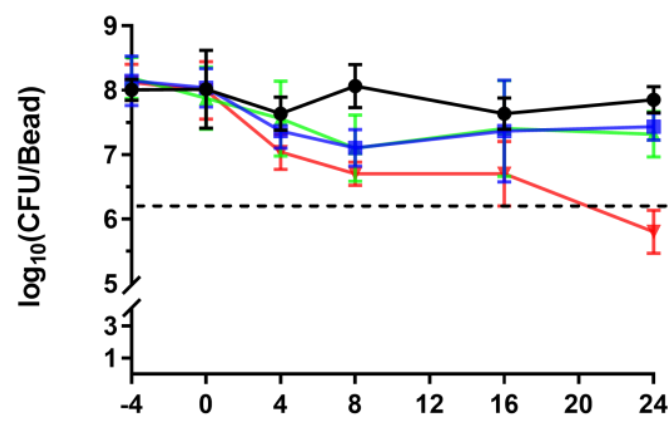

time (hr) p
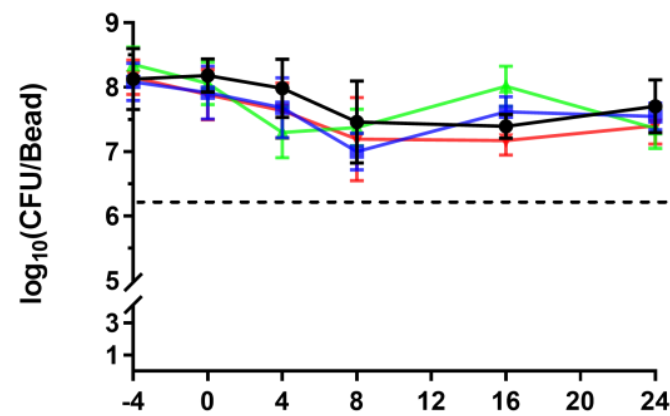

time (hr)

$\rightarrow$ Control - Imi $\neq$ Tob $\neq$ Imi+Tob

B

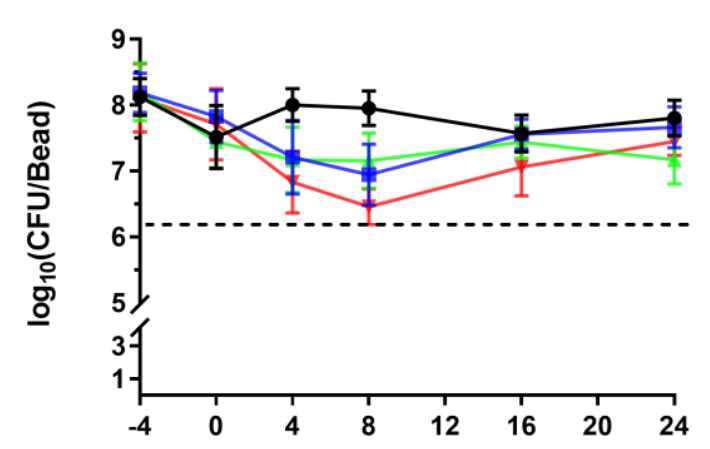

time (hr)

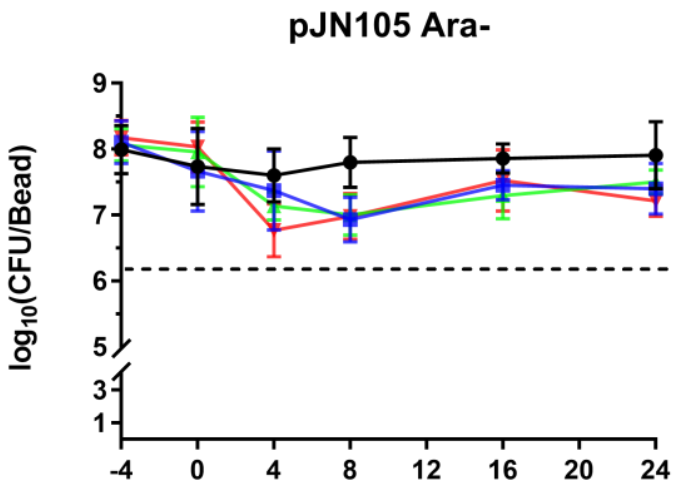

time (hr)

$\rightarrow$ Control $=$ Imi $\neq$ Tob $\neq$ Imi+Tob

Figure 2.5 Biofilm c-di-GMP mediated dispersal induced by arabinose enhances the synergistic antimicrobial effect of tobramycin (TOB) combined with imipenem (IMI) against $P$. aeruginosa PAO1/p $\mathrm{p}_{B A D}-y$ hjH. Residual bacterial load (CFU/beads) after exposing a 3-day grown biofilms of $P$. aeruginosa $P A O 1 / p_{B A D}-y h j H(\mathbf{A})$ or PAO1/pJN105 (B) treated (Ara+) or untreated (Ara-) with arabinose $1 \%$ to IMI and TOB alone or in combination with $8 \mu \mathrm{g} / \mathrm{mL}$. The dashed line represents $2 \log _{10}$ reduction in CFU/Bead relative to untreated controls, i.e. eradication of $99 \%$ of bacteria. Results are expressed as the average value of five independent experiments +/- STDEV, $(\mathrm{N}=5)$. 
The observation that Induced c-di-GMP mediated dispersal facilitates eradication of mature 3-day grown biofilms by the antibiotic combination was further confirmed by confocal microscopy (CLSM). Biofilms were grown using 8-well micro-slides; dispersal was induced for 4 hours with 1\% arabinose and was followed by the 24-hour antibiotic challenge (as monotherapy or combination of tobramycin and imipenem at $8 \mu \mathrm{g} / \mathrm{mL}$ ). Quantification of biofilm killing effect was conducted using syto9/PI stains, and the ratio of dead/live bacteria was calculated for each sample (Figure 2.6).

Following the imipenem-tobramycin combination exposure, a significant increase $(p<0.05)$ in the dead/live cell ratios $(0.77+/-0.12)$ was observed for PAO1/p $\mathrm{p}_{B A D^{-}} \mathrm{y} h \mathrm{H} H$ treated with arabinose (dispersed, Ara+), compared to the non-arabinose treated (non-dispersed, Ara-) biofilms $(0.56+/-0.13)$. Image analysis revealed that dispersed $\mathrm{p}_{B A D^{-}} \mathrm{yhjH}$ biofilms were also more susceptible to mono-treatment by tobramycin $(0.69+/-0.05)$ as compared to the non-dispersed control $(0.52+/-0.08, p<0.001)$ in this system. The most significant result $(p<0.0001)$ from comparing dead/live ratios is the killing effect seen in the combined antibiotic treatment following arabinose versus the control $(0.4+/-0.1)$ (Figure 2.6B). No significant differences in dead/live cell ratios were evident between treatments using the pJN105 strain. 
A
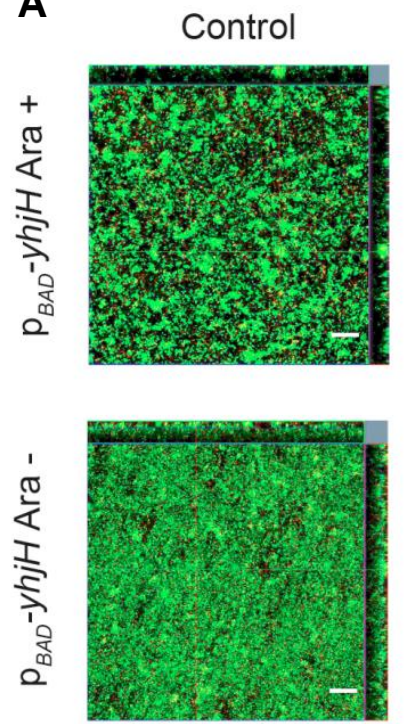

Control
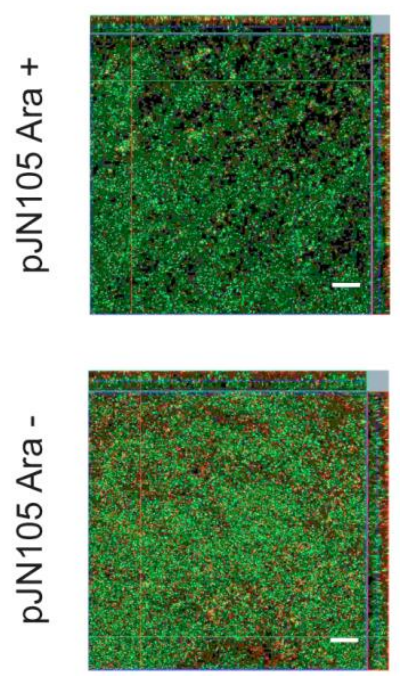

IMI
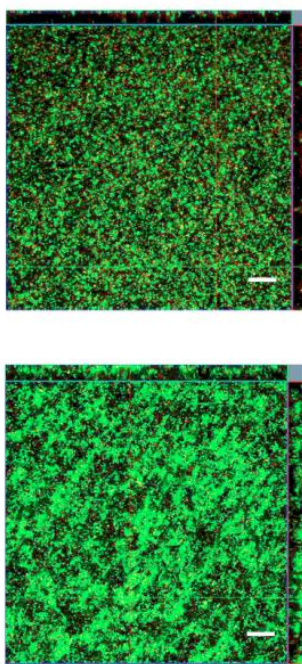

IMI
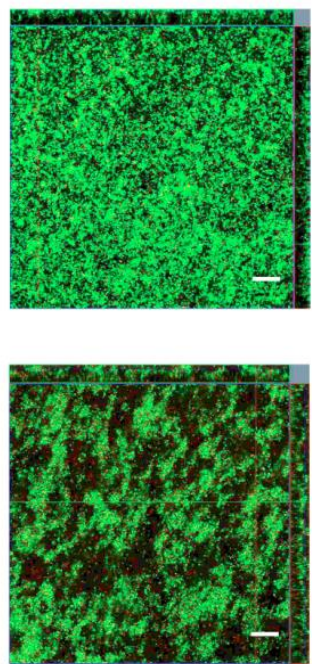

B

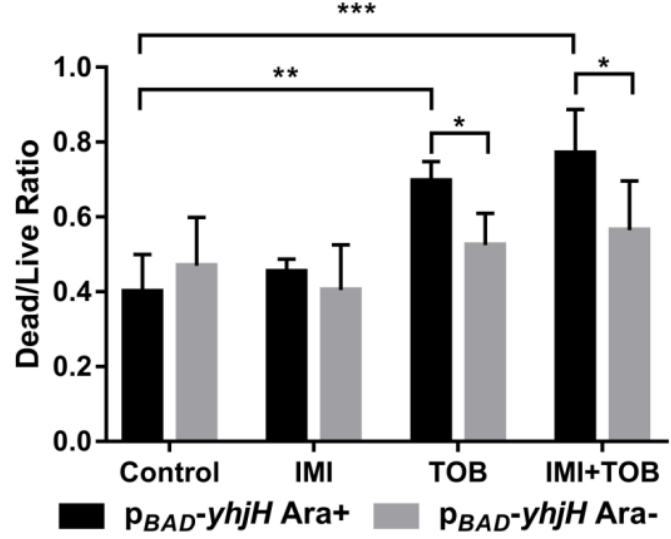

TOB
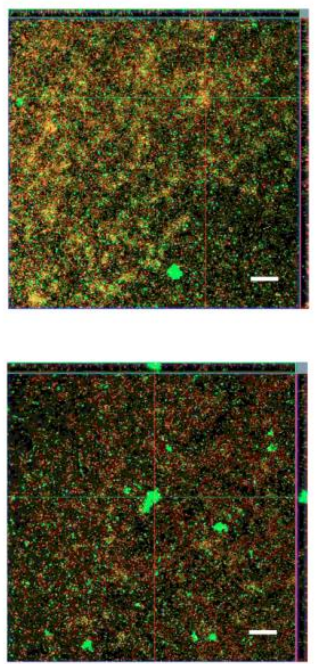

TOB
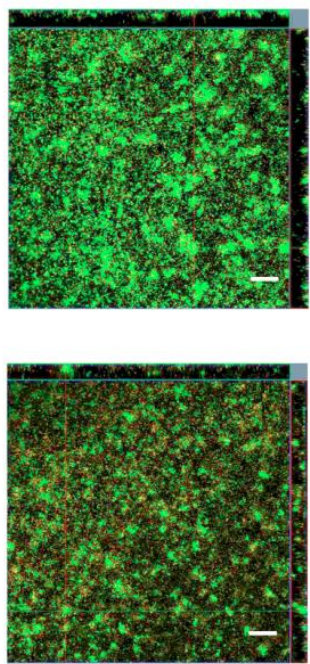

IMI+TOB
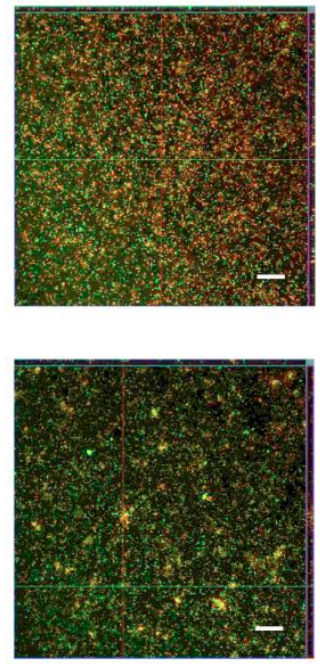

$\mathrm{IMI+TOB}$
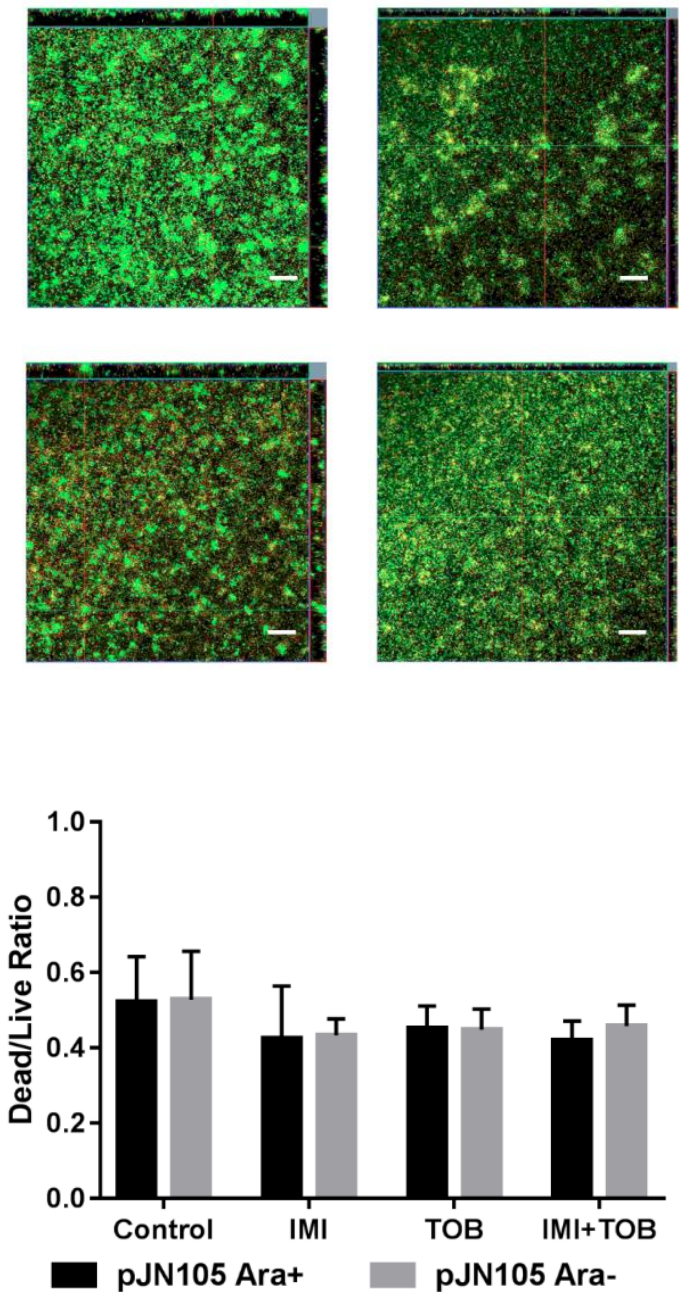

Figure 2.6 Confocal Microscopy (CLSM) images (20x objective) (A) of 3-day biofilms of $P$. aeruginosa strains $\mathrm{PAO} 1 / \mathrm{p}_{B A D}-y$ hjH and PAO1/pJN105 treated with arabinose $1 \%($ Ara + ) or untreated (Ara-) for 4 hours followed by 24 hours exposure to imipenem 
(IMI), tobramycin (TOB) or combination of IMI + TOB at $8 \mu \mathrm{g} / \mathrm{mL}$ each. Biofilms were stained with Syto9 and propidium iodide prior imaging. Dead and live cells appear red and green, respectively; the scale bar is $20 \mu \mathrm{m}$. Live-dead ratios for biofilms after 24 hour exposure to antibiotics are given (B); Images from five independent experiments $(\mathrm{N}=5)$ were analyzed to determine dead/live ratios for each condition. Results were analyzed using two-way ANOVA ( $p$-value interpretation: ${ }^{* * *}$ : $p<0.0001,{ }^{* *}: p<0.001$, $\left.{ }^{*}: p<0.05\right)$ and plotted as average values +/- STDEV.

\subsection{Discussion}

Using a $P$. aeruginosa construct $\mathrm{PAO} 1 / \mathrm{p}_{B A D^{-}} y h j \mathrm{H}$, it was demonstrated that c-di-GMP mediated biofilm dispersal induced by arabinose enhanced the killing efficacy of a combined antibiotic treatment (Figure 2.7 below). The findings are in agreement with published results for the combination of carbapenem agents with tobramycin (Pedersen, Pressler et al. 1987, Ciofu, Jensen et al. 1996, Hill, Rose et al. 2005, Dundar and Otkun 2010, Louie, Liu et al. 2013). It is known that the MIC's for both drugs are $0.5-1 \mu \mathrm{g} / \mathrm{mL}$ for susceptible strains $P$. aeruginosa it has been demonstrated in-vitro as well as in clinical isolates from CF patients that $P$. aeruginosa, exhibits high tolerance and eventually resistance is developed to monotherapies of both. The combination of carbapenems and tobramycin shows synergy but is effective at eradication of $99 \%$ of bacteria in high doses. The results presented here suggest that inducing biofilm dispersal via c-di-GMP depletion has the potential for a more potent antimicrobial efficacy by the use of lower drug concentrations. 


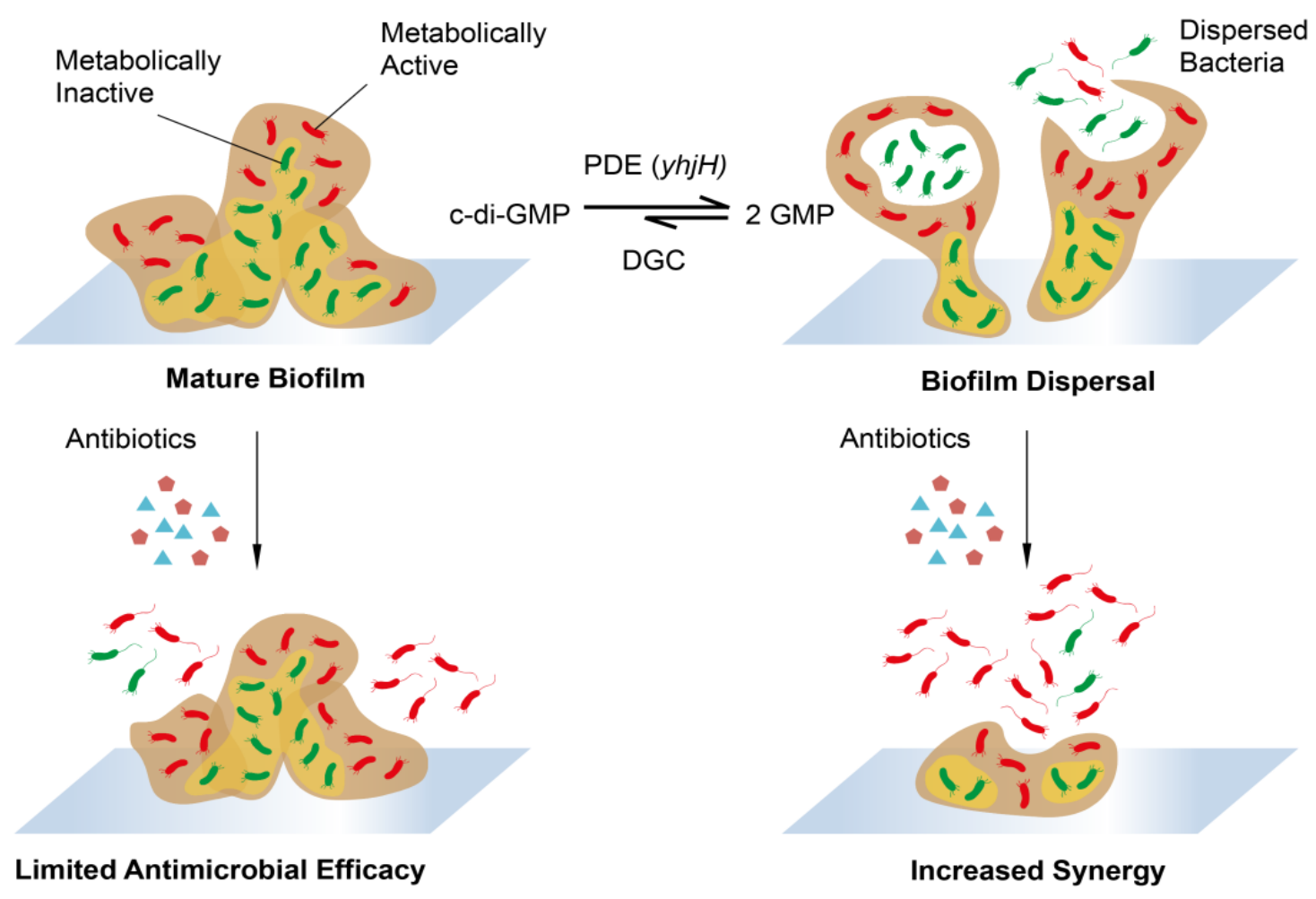

Figure 2.7 Model of proven hypothesis: Inducing dispersal through c-di-GMP depletion enhances the efficacy of combined antibiotic treatment (in red and blue) against mature biofilms. Red bacteria represent dead cells, and green bacteria represent remaining viable cells. Following induced dispersal by overexpression of yhjH PDE enhanced killing was evident, and the ratio of dead/live bacteria in biofilms also increased.

Experiments with planktonic cultures of PAO1 strains exhibited MBC of both antibiotics to be $2-4 \mu \mathrm{g} / \mathrm{mL}$. The relevant antibiotic concentrations in relation to biofilms of increased exponentially as biofilms were allowed to grow longer: 3-day grown biofilms used here, showed a significant increase in the concentrations of imipenem (of up to 1000 -fold) required to eradicate efficiently (MBEC $2048 \mu \mathrm{g} / \mathrm{mL}$ ), and 5 two-fold increase for tobramycin (MBEC $64 \mu \mathrm{g} / \mathrm{mL}$ ). These observations are in agreement with literature (Hengzhuang, Wu et al. 2011, Kapoor and Murphy 2018), that a 3-day P. aeruginosa biofilm 
model, can be considered "mature", usually exhibiting MBEC of $100-1000$ fold higher than MBC.

It is not uncommon for tobramycin to be administered once or three times daily (Bloomfield, Staatz et al. 2016), effective tobramycin plasma concentrations are usually not higher than $10 \mu \mathrm{g} / \mathrm{mL}$ (Burkhardt, Lehmann et al. 2006). For imipenem, it can reach $\mathrm{C}_{\max }$ levels of $\sim 64 \mu \mathrm{g} / \mathrm{mL}$, but blood plasma levels decline below $1 \mu \mathrm{g} / \mathrm{mL}$ after 6 hours (Lee, Kinzig-Schippers et al. 2010). In the work presented in this chapter, a concentration equal to twice the MBC, $8 \mu \mathrm{g} / \mathrm{mL}$ for both antibiotics was sufficient to eradicate $99 \%$ of the biofilm population in time-kill experiments, following induced c-di-GMP mediated dispersal. These antibiotic concentrations are thus in a relevant physiological range. The synergy of the combined treatment was not prominent in the sense that a $2 \log _{10}$ decrease in CFU versus tobramycin monotherapy was evident. The improvement to treatment due to c-di-GMP mediated biofilm dispersal was in accomplishing $\mathrm{MDK}_{99}$ of under 24-hours. Biofilms in undispersed samples and control samples were able to recover to above $10^{7} \mathrm{CFU} / \mathrm{mL}$ (7 $\log _{10}$ CFU/Bead) at the 24-hour time point.

The experiments presented here used a genetically engineered strain to demonstrate the proof of concept that induction of c-di-GMP mediated biofilm dispersal can sensitize biofilm cells to antibiotics and that sensitization is more pronounced for combination treatments. Therefore, the results presented here suggest that compounds that induce dispersal in non-engineered or w.t strain can be used as adjuvants to increase the efficacy of antibiotic combinations to treat infections. For example, nitric oxide (NO) donors (Barraud, J. Kelso et al. 
2015, Ren, Wu et al. 2016), QS or enzyme inhibitors (Rogers, Huigens et al. 2010, Kalan and Wright 2011), enzyme facilitated dispersal (Yu, Su et al. 2015, Gonzalez Moreno, Trampuz et al. 2017) and other techniques were shown to disperse biofilms.

$\mathrm{NO}$, in particular, functions through modulation of c-di-GMP concentration by higher PDE activity (Barraud, Schleheck et al. 2009), the same dispersal mechanism investigated here. One PDE is known to be activated by NO, $\mathrm{NbdA}$; other PDEs are activated by the chemotaxis sensor BdIA (Li, Petrova et al. 2014). NO is thus, related to metabolism, motility and dispersal, but also increase in virulence. NO has been shown to increase the efficacy of killing by tobramycin in clinical isolates from CF patients (Howlin, Cathie et al. 2017). When NO was used alone (as low as $500 \mathrm{nM}$ active compound) no more than $10 \%$ of the biofilm is dispersed, but when combined with antibiotic treatment, it facilitated possible synergy between tobramycin and other antibiotics. Findings from this chapter provide additional evidence that dispersal through c-di-GMP degradation increases the efficacy of tobramycin, and synergy between antibiotic combinations with tobramycin. The system used here requires a considerable time (4 hours) to induce dispersal, at high arabinose concentrations $(0.25-1 \% \mathrm{w} / \mathrm{v}, 16.6-66.6 \mathrm{mM})$; it is an innate problem with the araBAD expression system (Khlebnikov, Risa et al. 2000). Nonetheless, the effect seen by overexpression of the PDE YhjH was independent of the native PDE enzymes of $P$. aeruginosa. Regardless of which specific PDE involving mechanism is expressed, in response to NO exposure or by the construct presented here, inducing just one system facilitates partial dispersal which has the potential to increase antibiotic efficacy. 
CV staining has been suggested in a number of recent publications to be used as a standard proxy for biofilm susceptibility assays (Haney, Trimble et al. 2018, Kapoor and Murphy 2018). It is known that CV stains both live and dead bacterial populations, and all EPS components in varying degrees. These papers and others state that CV results show a high degree of variability, and are not-directly related to viability. CV staining results in this chapter could not be used to detect significant changes to the biofilm fraction of samples, not alone used for susceptibility.

The REMA assay seems to be an efficient way for a relatively rapid (4 hours incubation and measurement) to assess susceptibility, resistance and tolerance, with a useful detection range of approximately $10^{9}-10^{6} \mathrm{CFU} / \mathrm{mL}$ (Van den Driessche, Rigole et al. 2014, Kim and Jang 2017). When applied with the standard inoculum size of $10^{5}-10^{6} \mathrm{CFU} / \mathrm{mL}$ it is useful for MIC \& MBC measurement and high-throughput preliminary screening of MBC and MBEC values (Sandberg, Schellmann et al. 2009). Due to the detection limit, REMA is not suitable for assessment of persistence, estimated to be a $0.01 \%$ of the population (Brauner, Fridman et al. 2016). Routine measurements with REMA depend on comparison to negative and positive controls and depends on the user's threshold limit (Bueno 2014); in this research and others, 3 times the value of blank sample fluorescence was determined to indicate eradication (of down to $99.9 \%$ ). Kinetic experiments have been suggested to provide useful input, and more precise measurements down to a threshold of $10^{3} \mathrm{CFU} / \mathrm{mL}$, by producing and comparing results to specie-dependent activity curve of resazurin reduction (Van den Driessche, Rigole et al. 2014, Berditsch, Jäger et al. 2015). 
The CBD peg lid system is an efficient method for high-throughput exposure of virtually identical biofilm samples CFU/Peg approximately $10^{7}-10^{8}$ (Ceri, Olson et al. 1999). Here it was shown that the CBD could be utilized in tandem with REMA to identify promising antibiotic combinations. CBD was found here not to be suitable for routine high-throughput $\mathrm{CFU} / \mathrm{mL}$ count. The bead biofilm technique employed here has high reproducibility that can be used to measure parameters such as $\mathrm{CFU} / \mathrm{mL}$ in multiple conditions in relatively high throughput (Konrat, Schwebke et al. 2016). It is suggested that as long as beads remain submerged in media, it is possible to use as many beads as necessary as technical replicates (Sternberg, Bjarnsholt et al. 2014). All in all, these systems show the power and versatility of static in-vitro systems as powerful tools for fractionation of bacteria in different phases, planktonic, biofilm and dispersed and consequently high-throughput susceptibility testing.

Although the standardization of in-vitro experimentation and parameters for clinical biofilm related infections is in its early stage (Høiby, Bjarnsholt et al. 2015, Malone, Goeres et al. 2017, Haney, Trimble et al. 2018, Kapoor and Murphy 2018). Consistent with the literature, it is shown here that standard techniques as described in the CLSI (CLSI 2012) can be implemented and modified to be used as general conditions for biofilm focused experiments; some notable examples: CLSI suggests strict use of MHB as a default media, $\sim 5 \times 10^{5} \mathrm{CFU} / \mathrm{mL}$ inoculum, and use of methods such as broth microdilution, to allow comparison of parameters such as MIC/MBC across species (Reller, Weinstein et al. 2009), and for use of FIC indices to identify synergy of combinatorial treatment. All of the above were adopted here while developing the methodology for biofilm models. $\sum$ FIC estimation by broth microdilution 
checkerboard assay based on fluorescence such as REMA are usually performed with a minimum of five independent replicates $(\mathrm{N}=5)$ (Rand, Houck et al. 1993). By extension, the $\sum \mathrm{FBEC}$ of imipenem and tobramycin was calculated here in a checkerboard manner using the Calgary device, following the methodologies used for planktonic cells (e.g. $\sum$ FIC).

$\sum \mathrm{FIC}$ is a parameter used to identify the best synergistic drug combination in regards to $\mathrm{MIC}$, i.e. inhibiting planktonic bacteria. The $\mathrm{MIC}_{90}$ values of imipenem and tobramycin were 1 and $2 \mu \mathrm{g} / \mathrm{ml}$, respectively. The MBEC values of Imipenem and tobramycin as mono-therapy against 3-day mature biofilms were 2048 and $64 \mu \mathrm{g} / \mathrm{mL}$, respectively. The minimal concentration required for both drugs to observe a synergistic effect $\left(\sum \mathrm{FBEC} \leq 0.25\right)$ against both strains was 8-16 $\mu \mathrm{g} / \mathrm{mL}$, regardless of dispersal. This, in turn, corresponds by convention to FBEC of $0.004-0.008$ for imipenem, and $0.125-0.25$ for tobramycin. $\sum$ FBEC value of both is already $\leq 0.25$ ("highly synergistic") and becomes infinitesimally small and insignificant for a drop of a 1000-fold in concentration. It is not defined what differences in the parameter indicate below "high synergism". Additionally, results show here that dispersal and combined antibiotic treatment have higher efficacy; conventionally broth microdilution experiments have to agree at least $80 \%$ of the time (Rand, Houck et al. 1993, CLSI 2012). Because, the FBBEC parameter is not defined below 0.25 , further experiments were warranted to shed more light on the mechanism by which dispersal improves antimicrobial efficacy.

CLSM observations indicate that following c-di-GMP mediated dispersal tobramycin mono-treatment at $8 \mu \mathrm{g} / \mathrm{mL}$ shows significantly increased dead/live 
ratio $(0.7+/-0.05)$, as compared to the dispersed control $(0.4+/-0.1$, $p<0.001)$. This result suggests that tobramycin mono-treatment is sufficient to initiate killing with high efficacy. Killing is further enhanced $\sim 10 \%$ using the combination with imipenem but only for dispersed samples (dead/live ratio = $0.77+/-0.12, p<0.0001)$. No significant changes in killing between all three control groups were identified at $8 \mu \mathrm{g} / \mathrm{mL}$ for both antibiotics, alone and in combination. There were also no differences in average maximal biofilm thickness, regardless of dispersal or species: control for Ara+ or Ara- for either $\mathrm{p}_{B A D}$ yhjH or pJN105 averaged at $20+/-1.67 \mu \mathrm{m}$, and $9.95+/-0.40 \mu \mathrm{m}$ following combined treatments. It can be assumed then, that all antibiotic treatments resulted in approximately $50 \%$ reduction in biomass, out of which, only the combined antibiotic and dispersal treatment had the potential to achieve $>75 \%$ killing effect.

Time/kill experiments support that observation, monotherapy with either antibiotic for 8 hours following c-di-GMP mediated dispersal, already shows $90 \%$ killing $\left(1.1 \times 10^{7}\right.$ versus $\left.1.2 \times 10^{8} \mathrm{CFU} / \mathrm{Bead}\right),\left(\mathrm{MDK}_{90}\right)$; at that time point, the combined antibiotic treatment following dispersal shows 95\% killing $\left(5.01 \times 10^{6} \mathrm{CFU} / \mathrm{mL}\right) . \mathrm{MDK}_{99}$ then occurs only between $16-24$ hours with $\left(6.4 \times 10^{5}\right.$ versus $7.1 \times 10^{7} \mathrm{CFU} / \mathrm{mL}$ in control). A pharmacokinetic/ pharmacodynamics experiment, using mice infection models or the CDC bioreactor (Garey, Vo et al. 2009) is now warranted, to determine whether the recommended dosing thrapy regimens of this antibiotic combination in tandem with dispersal induction via this mechanism. 
In conclusion, the data presented in this chapter serves as a proof-of-concept for future development of dispersal agents to be used along with antimicrobial combinations. Inducing c-di-GMP mediated dispersal before antibiotic treatment allows for higher efficacy potential of antibiotic combinations at the start of a regimen. However, even a combined antibiotic treatment with dispersal cannot eradicate $100 \%$ of the bacterial cell population, and the remaining cells can regrow when the antibiotic pressure is removed. Additionally, those surviving biofilms may be enriched in genetic variants with enhanced antibiotic resistance (Drenkard and Ausubel 2002). The selective pressure effect of combined dispersal and antibiotics on biofilms has not tested to date. Thus, the following chapter focuses on whether induction of dispersal at different stages of biofilm formation affects the formation of resistant variants with or without the combination of antibiotic treatment. 


\section{Chapter 3: Impact of c-di-GMP Mediated Biofilm}

\section{Dispersal on the Rate of Tolerant Variant Development}

\subsection{Introduction}

In the previous chapter, c-di-GMP mediated biofilm dispersal has been shown to be a promising strategy to increase antimicrobial efficacy. This strategy, however, remains controversial as several reasons might delay its further usage in therapeutic treatments. First, following dispersal, bacteria might be released from the biofilm into the blood-stream, increasing the risk for acute and life-threatening systemic infections, such as sepsis and severe pneumonia (Fux, Stoodley et al. 2003). Second, "dispersed cells" consist of subpopulations with distinct phenotypes and genotypes, as compared to their planktonic counterparts, which might enhance their virulence mechanisms compared to planktonic cells. Only until recent years, dispersed cells were thought to harbor potentially higher antibiotic resistance than the typical planktonic cells. It has been shown that dispersed bacteria could eventually revert to the planktonic type of cells after multiple generations of growth (Chua, Liu et al. 2014). Finally, environmental selections occurring within the microbial community may result in the release of variant populations with reduced susceptibility to antimicrobial agents. This scenario is especially relevant following prolonged exposure to antimicrobial agents at sub-inhibitory concentrations, as it is often the case during treatments of chronic infections (Wright, Fothergill et al. 2013). 
Selection of variants with reduced antimicrobial susceptibility represents a significant threat, as they are typically associated with treatment failure and relapse of infections. Tolerant subpopulations refer to cells with the ability, inherited or not, to survive punctual exposure to high concentrations of antibiotics as a result of a slower metabolism. This phenotype is often observed with carbapenems, which require active cell wall synthesis to kill bacteria (Tuomanen, Cozens et al. 1986). For $P$. aeruginosa, it has been shown, that both stationary-phase bacteria and biofilm bacteria exhibit similar tolerance to antibiotics, and this was primarily attributed to the presence of persister cells (Spoering and Lewis 2001). Tolerant cells exhibit similar MIC values (concentrations required to kill $50 \%$ of the populations) as the susceptible parent population, but the $\mathrm{MDK}_{99}(\mathrm{MBC}$ or $\mathrm{MBEC})$ is significantly increased (Brauner, Fridman et al. 2016). In contrast, persisters are subpopulations of a clonal bacterial population with the non-heritable ability to survive prolonged exposure to high concentrations of antibiotics without any distinct resistance mechanisms (Lewis, Spoering et al. 2005). Persister cells exhibit similar MIC and $\mathrm{MDK}_{99}$ values as the susceptible parent population, but the minimal duration to eradicate $99.99 \%$ of the population, $\mathrm{MDK}_{99.99}$, is significantly higher (Brauner, Fridman et al. 2016).

Biofilms consist of bacterial cells at different stages of the development lifecycle, the population as a whole continually adapts to its surrounding environment. The multitude of mechanisms that enable biofilms to withstand exponentially higher antibiotic concentrations has been collectively termed as "Biofilm recalcitration" (Lebeaux, Ghigo et al. 2014). Although biofilms are considered as the ultimate protection of bacteria, stressful microenvironments 
can emerge from the high density of cells existing inside the microbial community. To survive the different micro-niches that characterize this everchanging environment, microbial cells have to evolve and adapt. These adaptation processes result in genotypic/phenotypic diversification, and several biofilm-derived morphological variants have been previously identified, especially for $P$. aeruginosa. Small-colony variants (SCVs) are the most described morphotype (Häußler, Tummler et al. 1999, Häußler 2004). SCVs of $P$. aeruginosa have been isolated from both in-vitro and in-vivo biofilms (Evans 2015). These bacteria exhibit enhanced surface attachment and biofilm formation capabilities, which are believed to facilitate the infection process eventually. Found during the dispersal process in $P$. aeruginosa PAO1 biofilm, this morphotype has been linked to the activity of Pf filamentous phages (Martínez and Campos-Gómez 2016). Other morphotypes include wrinkly variants which are characterized by overproduction of acetylated cellulose and Pel (Lebeaux, Ghigo et al. 2014). Sticky/mucoid variants, characterized by increased production of extracellular alginate or rugose variants which have been shown to auto-aggregate in liquid culture (D'Argenio, Calfee et al. 2002). Morphotype variants have been shown to differ in the affinity to bind the dye Congo-Red, making them easier to identify (Kirisits, Prost et al. 2005, Ueda and Wood 2009).

In the past decades, a particular emphasis has been put on MDR bacterial infections, for which the arsenal of chemotherapies is dramatically diminishing (Izadpanah and Khalili 2015). Colistin is considered the last resort antibiotic against Gram-negative organisms and is routinely used in chronic and difficult to treat MDR bacterial infections, such as cystic fibrosis (CF) (Jansen, Mahrt et 
al. 2016). Administered as either mono-treatment or in combinations to yield synergistic potential (Martis, Leroy et al. 2014), resistance to colistin remains seldom (Liu, Wang et al. 2016). Non-heritable and inherited abilities to survive colistin exposures have, however, been reported; the latter being associated to modifications of Lipid-A, its biological target, which is found in the outer Gram-negative membranes (Lee, Park et al. 2016). Due to high fitness costs, mutations are known to revert once colistin stress subsides. Thus, $P$. aeruginosa exhibits transient colistin resistant phenotypes. Lastly, it has been shown that $P$. aeruginosa dispersal through NO shows potential synergy with colistin treatment, in in-vivo experiments in a catheter model (Ren, Wu et al. 2016). The similarities between NO mediated dispersal to the $\mathrm{p}_{B A D^{-}} y$ hjH induced dispersal imply further investigation into increasing the efficacy of this last resort drug.

The objective of this work was to investigate biofilm-associated morphotypes emerging secondary to colistin exposure, and the effect of induced c-di-GMP mediated dispersal might have on their development.

\subsection{Methods}

Bacterial strains: The strain of $P$. aeruginosa used in this section is PAO1/p ${ }_{B A D^{-}} y h j H:: G F P ;$ a plasmid carrying $y h j H$ under the araBAD promoter. The strain also carries a gene encoding for green fluorescent protein (GFP), inserted into PAO1 w.t genomic DNA, with miniTn7-Gm ${ }^{r}-p_{p m r}-g f p$ (Chua, Tan et al. 2013). The strain is kept at $-80^{\circ} \mathrm{C}$ and routinely cultured onto LB Agar supplemented with gentamicin sulfate at $60 \mu \mathrm{g} / \mathrm{mL}$. 
Chemical agents: LB Agar, Miller recipe (BD Difco) and MHB or MHA (SigmaAldrich), Tryptone, Agar (BD) Congo-red (Sigma-Aldrich) were used. Antimicrobial agents used throughout this chapter were gentamicin-sulfate and colistin-sulfate (MP Biomedicals).

Biofilm growth and quantification of colistin MBEC: Biofilms were grown on $5 \mathrm{~mm}$ glass beads (Merck) for 1 to 3 days, as described in chapter 2. Two beads (representing two technical replicates) were placed into each well of a 24-well microtiter plate (NUNC ${ }^{\mathrm{TM}}$, Thermo-Fischer). MHB media, containing $0.25 \%$ arabinose (Ara+) and non-arabinose containing (Ara-), were used to grow biofilms under constant dispersal induction or without. Every 24 hours, samples were washed with $0.9 \% \mathrm{NaCl}$, before replenishing the medium in all tested conditions. Beads were collected daily in $0.9 \% \mathrm{NaCl}$; biofilm bacteria were harvested by subjecting the beads to a series of 5 times 10 -second sonication (at $37 \mathrm{kHz}$ ) and 10-second vortex. Bacterial suspensions were subsequently serially diluted $\left(10^{3}-10^{5}\right)$ in $0.9 \% \mathrm{NaCl}$, and $10 \mu \mathrm{L}$ aliquots plated on MHA plates containing $0-64 \mu \mathrm{g} / \mathrm{mL}$ colistin. Following 24-hour incubation at $37^{\circ} \mathrm{C}, \mathrm{CFU} /$ Bead were calculated for each concentration, the colistin MBEC was calculated as the concentration resulting in $99 \%$ killing relative to untreated control (that result in $2 \log _{10}$ (CFU/Bead) killing).

Identification of morphological variants: Biofilms were grown for 1-3 days and harvested as described above in the following four experimental conditions: 
- MHB containing $0.25 \%(\mathrm{w} / \mathrm{v})$ arabinose "Ara+ Control"

- MHB containing $0.25 \%(\mathrm{w} / \mathrm{v})$ arabinose + colistin at $8 \mu \mathrm{g} / \mathrm{mL}$ "Ara+ Colistin"

- Arabinose free MHB "Ara- Control"

- Arabinose free MHB + colistin at $8 \mu \mathrm{g} / \mathrm{mL}$ "Ara- Colistin"

The development of variants due to exposure to antibiotic stress by colistin is evaluated using the Congo-red agar plate assay. $100 \mu \mathrm{L}$ of each dilution were then spread plated on Congo-red agar plates as previously described (Friedman and Kolter 2004). Congo-red agar was prepared using a solution consisting of an autoclaved solution of $1 \%$ Tryptone (10 gr $/ \mathrm{L})$ and $1 \%$ Agar (10 gr/L). $1 \mathrm{~mL}$ of each of the following stock solutions are added: $40 \mathrm{mg} / \mathrm{mL}$ Congo-Red, $10 \mathrm{mg} / \mathrm{mL} \mathrm{MgCl} \cdot 6 \mathrm{H}_{2} \mathrm{O}$ and $20 \mathrm{mg} / \mathrm{mL} \mathrm{CaCl} \cdot 2 \mathrm{H}_{2} \mathrm{O}$ (to achieve same salt concentration as in MHB). Variants were selected based on their susceptibility to colistin. A series of Congo-red agar plates containing increased concentrations of colistin $(0,4,8,16,32,64 \mu \mathrm{g} / \mathrm{mL})$ were prepared. Plates were then incubated at $37^{\circ} \mathrm{C}$ for 24 hours and then left for additional 48 hours at room temperature to allow for distinguishable variant growth on the basis of the morphology of the colonies. Experiments were performed 4 times to ensure reproducibility of results $(\mathrm{N}=4)$, and thus, each of the followup experiments were conducted in 4 biological replicates. Selected variants were then picked with a sterile toothpick, inoculated into $2 \mathrm{~mL}$ of MHB media and left 8 hours in a $37^{\circ} \mathrm{C}$ shaking incubator (200 rpm). Once samples reached similar turbidity at $0.010 . D_{600}$, bacterial cultures were used for additional phenotypic assays. 
Difference in variant biofilm production: Biofilm formation was quantified using crystal violet (CV, Sigma Aldrich) staining method, as previously described (O'Toole 2011, Chua, Hultqvist et al. 2015). Briefly, 24-well microtiter plates were utilized, $500 \mu \mathrm{L}$ of fresh MHB broth were added in each well of 24 -well microtiter plates. Each well was inoculated with $500 \mu \mathrm{L}$ of the variants grown up to $0.01 O . \mathrm{D}_{600}$. The microtiter plate was incubated overnight at $37^{\circ} \mathrm{C}$. The following days, samples were washed and stained with CV $(0.01 \%$, vol $/ \mathrm{vol}$ in $\mathrm{dH}_{2} \mathrm{O}$ ). After 30 minutes, the excess of $\mathrm{CV}$ stain was removed, rinsed with $\mathrm{dH}_{2} \mathrm{O}$, dried off and solubilized in $100 \%$ ethanol. The absorbance of each sample was measured at $590 \mathrm{~nm}$ using a microplate reader (Infinite Pro 200, Tecan). Three technical replicates were performed for each variant selected and results are presented as mean $+/$ - Standard Deviation (STDEV) $(\mathrm{N}=4)$.

Variant antimicrobial susceptibility profile: MIC values were determined using 96-well microtiter plate method, as performed earlier. A gradient of colistin (0-32 $\mu \mathrm{g} / \mathrm{mL})$ was created in two-fold dilution manner vertically in each plate. Different variants were inoculated in columns 1-12 in duplicates; a total of 6 samples were tested in each plate. Samples were allowed to grow at $37^{\circ} \mathrm{C}$. After 24 hours, growth was determined based on turbidity observed following visual inspection and O.D $D_{600}$ measurement. The MIC values were defined as the lowest concentrations for which no visible growth was observed or via O. $D_{600}$ measurement if sample turbidity was no more than 3 times the turbidity of control wells.

Variant motility assay: Agar plates were prepared to assay motility (Chow, Gu et al. 2011, Chang, Krishnan et al. 2014). Agar consisted of $0.5 \%$ peptone 
(5 gr/L), 0.2\% yeast extract (2 gr/L) and agar at $0.3 \%$ (3 gr/L, for swimming motility), 0.5\% (5 gr/L, for swarming motility) and 0.9\% Agar (9 gr/L, twitching motility). Following autoclaving, $1 \%$ Glucose (w/v, final) was added to the prepared agar before pouring plates in Petri dishes (60 mm standard plates). Inoculation with a sterile toothpick was performed differently for each type of plate: For swarming, the toothpick was placed at the surface of the agar; for swimming, the toothpick was inserted halfway in the agar, whereas for twitching the toothpick penetrated all the way through the agar down to the bottom of the plate. Plates were left in the $30^{\circ} \mathrm{C}$ incubator for up to 10 hours (swimming), 12 hours (swarming) and 48 hours (twitching). Swimming and swarming plates were imaged directly using the gel imaging station. The GFP fluorescence of cells was utilized to image for better estimation of the colony size as compared to UV which is used in most cases. Images were later analyzed using ImageJ software (https://imagej.nig.gov/ij) to calculate the area (in $\mathrm{mm}^{2}$ ) occupied by bacterial colony on each plate. Twitching motility required careful removal of the agar and CV staining. After proper de-staining of unbound CV by washing with tap water and drying off the plate, a ruler was used to measure the diameter (in $\mathrm{mm}$ ) of the twitching zone.


Congo-red agar plates were collected manually using a mechanical counter; results were collected and computed in Excel 2010. Calculated CFU/bead results and variant development were analyzed for statistical differences and plotted using Prism Graphpad7. Statistical significance was assessed by multiple comparison two-way ANOVA using in software algorithm under conservative Tukey test at $95 \%$ confidence interval. 


\subsection{Results}

Colistin MBEC values for different stages of biofilm growth

The bead biofilm system was used to determine PAO1/p $\mathrm{p}_{B A D}-y h j H:: G F P$ MBEC of colistin for 1-3 day grown biofilms under continuous induced dispersal (Figure 3.1). Untreated biofilm control samples reached cell densities of $>2 \times 10^{6} \mathrm{CFU} / \mathrm{Bead}$, continuous induced dispersal (Ara+) without added colistin did not lead to considerable difference in CFU/Bead numbers $\left(2.3-1.9 \times 10^{6}\right.$ $\mathrm{CFU} /$ Bead, on average for 1-3 day samples). For 1-day grown biofilms the MBEC of colistin was already at $64 \mu \mathrm{g} / \mathrm{mL}\left(2.1 \times 10^{3} \mathrm{CFU} / \mathrm{Bead}+/-2.3 \times 10^{2}\right)$, for the Ara+ 1-day biofilm the MBEC of colistin was between $32-64 \mu \mathrm{g} / \mathrm{mL}$ $\left(9.3 \times 10^{3}-2.6 \times 10^{3} \mathrm{CFU} /\right.$ Bead $\left.+/-2.25 \times 10^{2}\right)$. Similarly, for 2-day grown biofilms the MBEC for Ara- samples was $64 \mu \mathrm{g} / \mathrm{mL}\left(9.5 \times 10^{3} \mathrm{CFU} /\right.$ Bead $\left.+/-3.6 \times 10^{3}\right)$, while a sharper drop in CFU/Bead is evident for the Ara+ samples at 32-64 $\mu \mathrm{g} / \mathrm{mL}\left(2.6 \times 10^{4}-2.6 \times 10^{3} \mathrm{CFU} /\right.$ Bead $\left.+/-2.8 \times 10^{2}\right)$. MBEC for 3-day biofilms is $>64 \mu \mathrm{g} / \mathrm{mL}$ for Ara- samples $\left(3.8 \times 10^{4}+/-7.5 \times 10^{3}\right)$, (probably 1 dilution); while for Ara+ samples MBEC is still $64 \mu \mathrm{g} / \mathrm{mL}\left(1.7 \times 10^{4} \mathrm{CFU} / \mathrm{Bead}+/-5.1 \times 10^{3}\right)$.


Figure 3.1 CFU/Bead count on MHA plates containing 0-64 $\mu \mathrm{g} / \mathrm{mL}$ colistin for PAO1/p $p_{B A D}-y h j H:: G F P$ biofilms grown on $5 \mathrm{~mm}$ beads for $1,2 \& 3$ days in MHB with $0.25 \%$ arabinose (Ara+) or without (Ara-). Results are from three independent experiments and presented as average values +/- STDEV $(\mathrm{N}=3)$. 
A clear shift is seen in 3-day biofilms, as higher numbers of resistant population bacteria with $10^{4}-10^{5} \mathrm{CFU} /$ Bead are evident at $64 \mu \mathrm{g} / \mathrm{mL}$, with constant Ara+ treatment or without. Arabinose treatment does not seem to significantly affect the proportions of antimicrobial resistant subpopulations as no difference was observed in biofilms grown over the course of 3-days between Ara+ and Ara- samples. Consolidating results for planktonic cultures from the previous chapter (Table 2.1), the relevant concentrations for PAO1/p $\mathrm{p}_{B A D^{-}} \mathrm{yhjH}$ are MIC of $0.5-1 \mu \mathrm{g} / \mathrm{mL}$ and MBC of $2-4 \mu \mathrm{g} / \mathrm{mL}$. Biofilms thus exhibit 4-6 twofold lower colistin susceptibility depending on the maturity of the sample, similar trends to this result are seen in literature (Hengzhuang, Wu et al. 2011).

\section{Variant populations, morphotypes and frequency}

Following preliminary experiments, the concentration of $8 \mu \mathrm{g} / \mathrm{mL}$ of colistin was chosen to represent a sub-optimal antibiotic concentration for continuous exposure to biofilms. It is equal to twice the MBC, is below the MBEC for 1-3 day grown biofilms and is in the range of the average $C_{\max }$ plasma concentration achievable during in-vivo treatment ( $\mathrm{Li}$, Coulthard et al. 2003).

The Congo-red agar plate method allowed identification of 3 different morphology phenotypes (Figure 3.2) and categorized as Smooth, Spreader and Small. Smooth and spreader colonies were large colonies, the latter with a less distinct colony outline. Small colonies were mostly pinkish and tiny. 


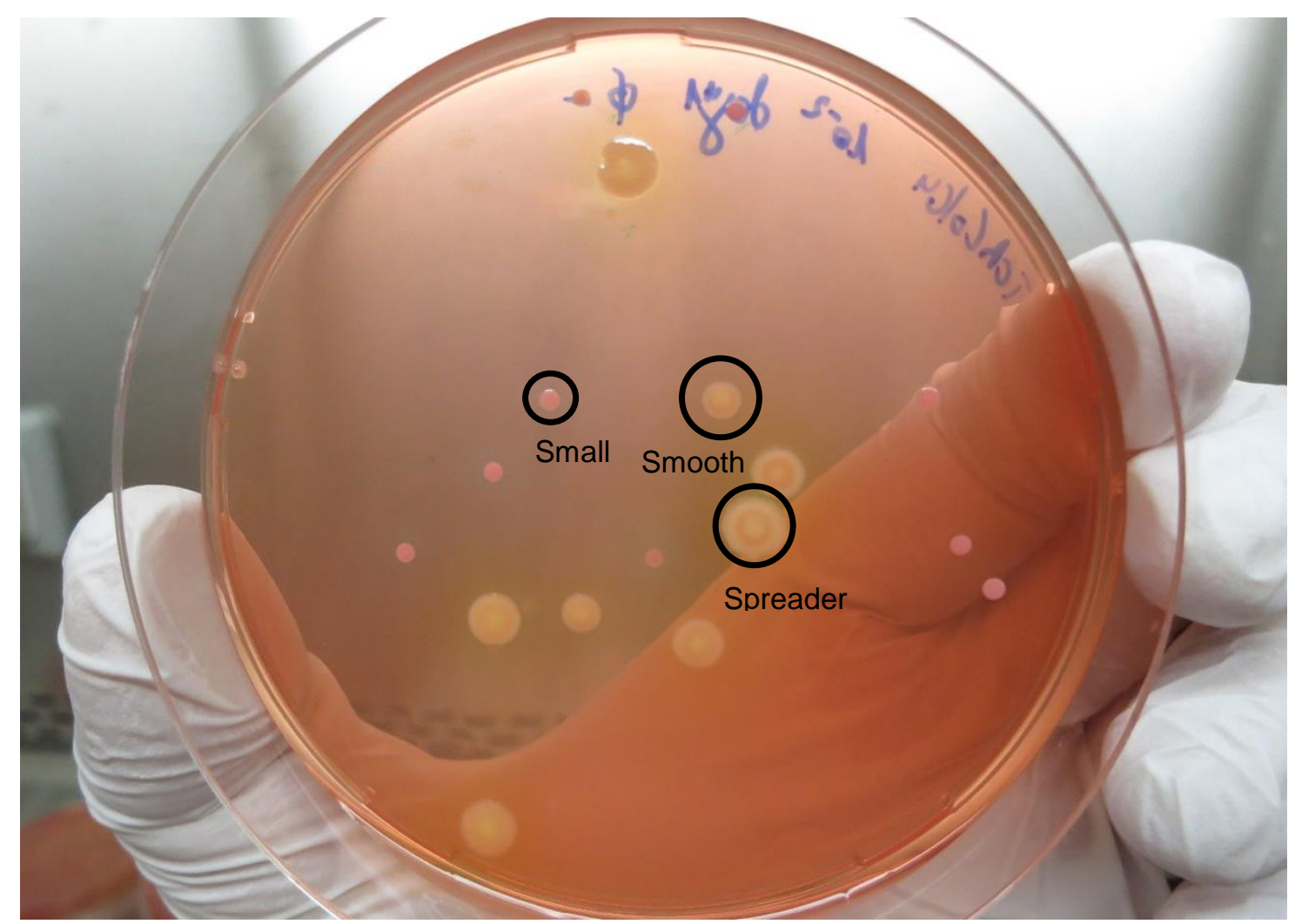

Figure 3.2 Example for colony morphologies observed on Congo-red containing agar plates, following 24 hours incubation in $37^{\circ} \mathrm{C}$ and 48 hours at room temperature. In this example a 1-day grown biofilm that was not treated with either arabinose $0.25 \%$ or colistin $8 \mu \mathrm{g} / \mathrm{mL}$ ("Control Ara-"). Serially diluted five times (CFU x $10^{6}$ final), was plated onto Congo-red agar plate supplemented with $4 \mu \mathrm{g} / \mathrm{mL}$ colistin. 
Each of these variant populations (smooth, spreader \& small) CFU was enumerated and plotted versus the 4 growth conditions (+/- arabinose, $+/$ - colistin $8 \mu \mathrm{g} / \mathrm{mL}$ ). Variant development was plotted over number of growth days (Figure 3.3); using interleaved bar charts over $0-64 \mu \mathrm{g} / \mathrm{mL}$ range of colistin concentration.



Figure 3.3 Development of population variants in $\log _{10}(\mathrm{CFU} / \mathrm{Bead})$ (Smooth, Spreader \& Small) for 1-3 days of growth, in different growth conditions: MHB only (Control), $8 \mu \mathrm{g} / \mathrm{mL}$ colistin, with or without $0.25 \%$ arabinose (Ara+ / Ara-). 
X-axis represents colistin concentration in plates $[\mu \mathrm{g} / \mathrm{mL}]$. Experiments conducted in quadruplicates, $(\mathrm{N}=4)$ expressed as the average value of four independent experiments +/- STDEV.

Results were not normalized to allow for actual CFU/Bead numbers to be conveyed. As shown, at the highest colistin concentration of $64 \mu \mathrm{g} / \mathrm{mL}$, only small colonies growth was evident; the fraction that survives constitutes $0.01 \%$ of the population $\left(10^{3}-10^{4} \mathrm{CFU} / \mathrm{Bead}\right)$. There were no significant differences in variant development due to different growth conditions for biofilms grown for 1 day up to 3 days. Thus, continued induced dispersal did not cause enrichment for the tolerant small colony morphotype.

\section{Phenotypes of variants}

In an attempt to further investigate differences between the variant populations, 20 colonies within the 3 morphotype categories growing on $32 \mu \mathrm{g} / \mathrm{mL}$ colistin plates were selected and tested for various phenotypic assays (colistin MIC, biofilm production, and surface motility); results are summarized in table 3.1 versus the parent strain (termed "wt" in table).

Although the variants were selected on colistin $32 \mu \mathrm{g} / \mathrm{mL}$ agar, most of the variants $(16 / 20)$ exhibited increased or similar susceptibility to colistin as compared to the w.t population (MIC ranging between $<1$ to $2 \mu \mathrm{g} / \mathrm{mL}$ ). When strains were recultured and MIC examined by the broth microdilution methods, it is evident that bacteria outside of the biofilm context become more susceptible. 
Table 3.1 Phenotypic properties of 20 selected colonies from the 3 different morphotype categories picked from $32 \mu \mathrm{g} / \mathrm{mL}$ colistin plates.

\begin{tabular}{|c|c|c|c|c|c|c|c|c|}
\hline \multirow{2}{*}{$\begin{array}{c}\text { Phenotype } \\
\text { wt }\end{array}$} & \multirow{2}{*}{$\begin{array}{c}\begin{array}{c}\text { Colistin MIC } \\
{[\mu \mathrm{g} / \mathrm{mL}]}\end{array} \\
1-2 \\
\end{array}$} & \multirow{2}{*}{\begin{tabular}{|c|}
$\begin{array}{l}\text { Biofilm Production CV } \\
\left.\text { Abs }_{590} \text { [Relative to } \mathbf{w t}\right]\end{array}$ \\
1.00 \\
\end{tabular}} & \multicolumn{2}{|c|}{$\begin{array}{c}\text { Swimming } \\
{\left[\mathrm{mm}^{2}\right]}\end{array}$} & \multicolumn{2}{|c|}{$\begin{array}{c}\text { Swarming } \\
{\left[\mathrm{mm}^{2}\right]}\end{array}$} & \multicolumn{2}{|c|}{$\begin{array}{l}\text { Twitching } \\
\text { [mm] }\end{array}$} \\
\hline & & & 95.44 & + & 37.76 & + & 7 & + \\
\hline Smooth & $0-1$ & 1.17 & 172.05 & + & 78.46 & + & 9 & + \\
\hline Smooth & $0-1$ & 0.78 & 133.83 & + & 35.39 & + & 15 & ++ \\
\hline Smooth & $0-1$ & 1.46 & 369.87 & ++ & 49.34 & + & 9 & + \\
\hline Smooth & $0-1$ & 1.59 & 83.17 & + & 16.87 & + & 9 & + \\
\hline Smooth & $4-8$ & 1.14 & 133.71 & + & 30.65 & + & 10 & ++ \\
\hline Smooth & $4-8$ & 1.35 & 9.87 & - & 6.83 & + & 4 & - \\
\hline Spreader & $0-1$ & 0.88 & 68.54 & + & 28.51 & + & 4 & - \\
\hline Spreader & $0-1$ & 1.68 & 53.52 & + & 14.13 & + & 6 & + \\
\hline Spreader & $0-1$ & 1.57 & 96.44 & + & 26.91 & + & 3 & - \\
\hline Spreader & $0-1$ & 1.80 & 42.12 & + & 15.82 & + & 4 & - \\
\hline Spreader & $0-1$ & 1.52 & 125.02 & + & 324.24 & ++ & 2 & - \\
\hline Spreader & $1-2$ & 1.30 & 7.36 & - & 1.05 & - & 4 & - \\
\hline Spreader & $4-8$ & 1.08 & 1120.12 & ++ & 24.85 & + & 6 & + \\
\hline Small & $0-1$ & 0.64 & 120.66 & + & 361.69 & ++ & 5 & - \\
\hline Small & $0-1$ & 1.17 & 53.07 & + & 22.16 & + & 7 & + \\
\hline Small & $0-1$ & 1.80 & 91.51 & + & 16.67 & + & 4 & - \\
\hline Small & $0-1$ & 1.29 & 104.70 & + & 24.37 & + & 9 & + \\
\hline Small & $0-1$ & 0.69 & 163.70 & + & 14.83 & + & 6 & + \\
\hline Small & $1-2$ & 1.18 & 178.39 & + & 16.49 & + & 8 & + \\
\hline Small & $4-8$ & 1.39 & 48.37 & + & 5.81 & - & 5 & - \\
\hline
\end{tabular}

Similarly, $17 / 20$ of the tested variants demonstrated increased biofilm production relative to the w.t. Examining surface motility; variants demonstrated increased or reduced motility independent of their morphotype. Decreased motility was the most consistent in the Spreaders with 5 out of 7 tested colonies exhibiting reduced swimming, swarming and twitching zones as compared to w.t phenotype. Example swimming and swarming motilities phenotypes can be seen in figure $\mathbf{3 . 4}$. 
Swimming $\left(\mathrm{mm}^{2}\right)$

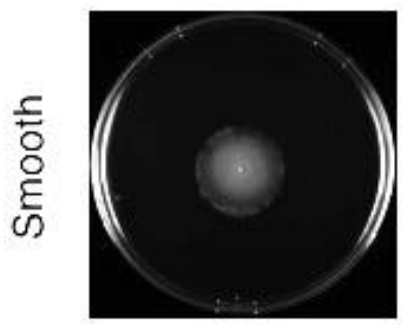

133.83

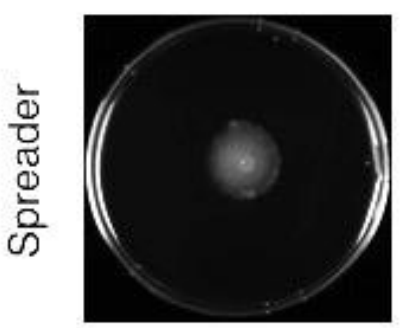

96.44

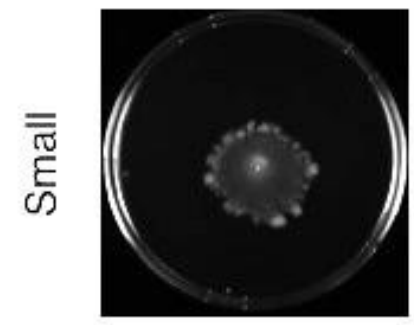

178.39

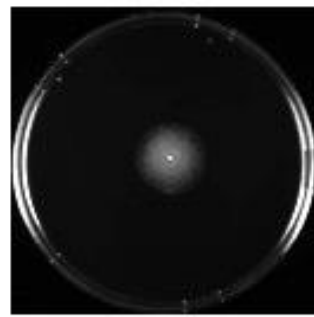

83.17

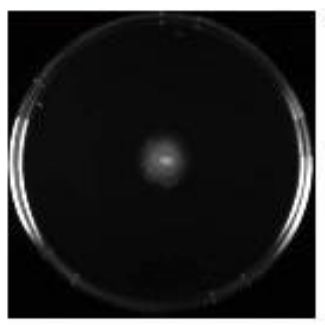

42.12

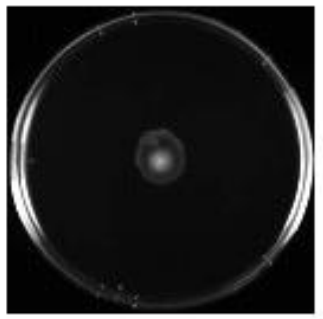

48.37

Swarming $\left(\mathrm{mm}^{2}\right)$

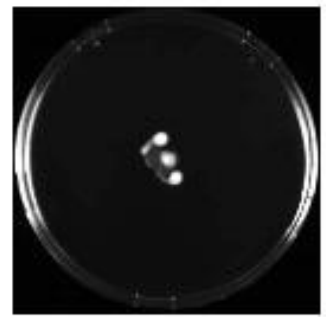

35.39

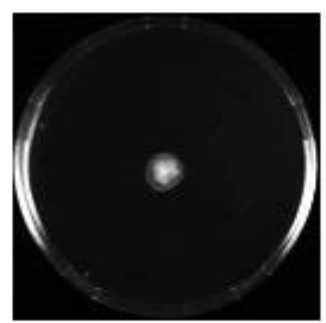

26.91

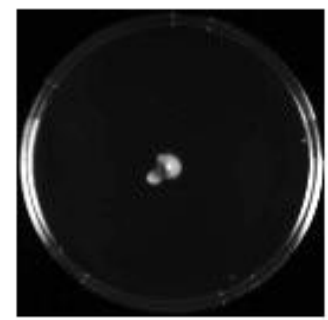

16.49

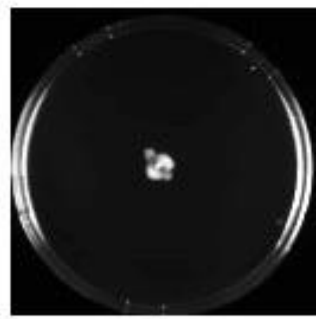

16.87



15.82



5.81

Figure 3.4 Example motility patterns of selected Smooth, Spreader and Small colony variants originating from the parent PAO1/p ${ }_{B A D}-y h j H:: G F P$. Swimming (Left) and Swarming (Right) agar assays have been used to evaluate the motility phenotypes of selected variants, here two examples of each morphotype have been shown. The surface coverage $\left(\mathrm{mm}^{2}\right)$ has been analyzed using ImageJ software on images acquired in gel imaging station utilizing expressed GFP fluorescence.

\section{The effect of extended growth on variant development}

Since no significant differences in the frequency of variants could be identified, more extended duration, preliminary experiment, growing PAO1/p BAD- $_{\text {- }} h j H:: G F P$ under the same treatment groups was conducted. Biofilms were grown for 7 and 12 days and the resulting variant CFU/Bead ratios were compared to 3-day grown biofilms (Figure 3.5). Though these results are exploratory at this stage, and more experiments are warranted, it is 
clear that the biofilms exhibit higher tolerance to colistin when allowed to grow up to this point. The highest colistin concentration exposure in this experiment, $64 \mu \mathrm{g} / \mathrm{mL}$, resulted only in less than a $\sim 2 \log _{10}$ decrease in CFU/Bead (For instance, $2.7 \times 10^{5} \mathrm{CFU} /$ Bead at $64 \mu \mathrm{g} / \mathrm{mL}$ versus $2.1 \times 10^{7} \mathrm{CFU} /$ Bead at $0 \mu \mathrm{g} / \mathrm{mL}$, for Ara- Control treatment for 7-day biofilms). The most evident difference between groups occurred in biofilms that were grown for 12 days in Ara+ conditions. When plated on $64 \mu \mathrm{g} / \mathrm{mL}$ colistin, colonies of all three morphotypes could be detected. This result might point at the experimental duration necessary for development and proliferation of biofilm-specific mutations that have higher fitness under colistin stress. 


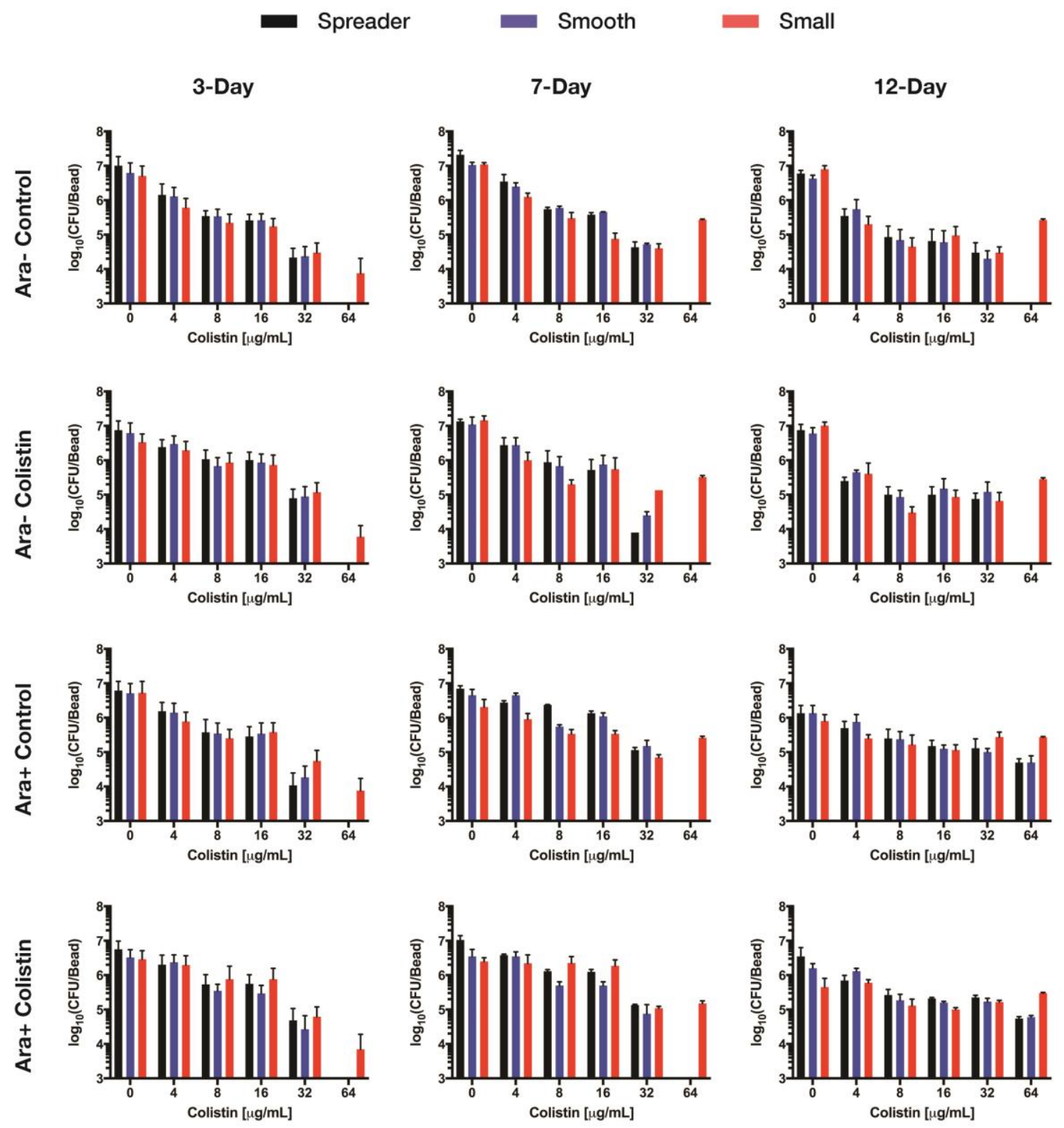

Figure 3.5 Preliminary development of population variants results as $\log _{10}$ (CFU/Bead) (Smooth, Spreader \& Small) for 3-12 days of growth, in different growth conditions: MHB only (Control), $8 \mu \mathrm{g} / \mathrm{mL}$ colistin, with or without $0.25 \%$ arabinose (Ara+ / Ara-). X-axis represents colistin concentration in plates $[\mu \mathrm{g} / \mathrm{mL}]$. For 7-day and 12-day grown biofilms, only one experiment was conducted once with technical duplicates. 


\subsection{Discussion}

C-di-GMP mediated biofilm dispersal has proven to be a promising strategy to increase antimicrobial efficacy in-vitro. However, controversy remains on its potential application in-vivo (Fleming and Rumbaugh 2017). One of the reasons is the increased risk of sepsis caused by dispersed cells released into the bloodstream. Although these dispersed cells are known to exhibit reduced susceptibility to antimicrobial agents as compared to the susceptible population, they also quickly revert to planktonic mode, suggesting that they do not represent a therapeutic challenge if the bloodstream infection remains manageable. Another reason for the controversy is the potential release of variant morphotypes (Fux, Stoodley et al. 2003, Bjarnsholt, Tolker-Nielsen et al. 2010, Lebeaux, Ghigo et al. 2014). These biofilm-derived morphotypes, selected by environmental stresses as a survival strategy, are of more significant concern as they exhibit increased resistance and/or virulence. The motivation for the present research was to assess whether in-vitro induced dispersal together with colistin treatment increases the risk of release of morphotype variants.

The previously described construct of $P$. aeruginosa, PAO1/p $\mathrm{p}_{B A D}-y h j H$ was used as a model organism for proof-of-concept. Phenotypic variants were identified based on their morphotypes on Congo-red agar supplemented or not, with increasing concentrations of colistin (up to $64 \mu \mathrm{g} / \mathrm{mL}$ ). Consistent with the literature, 3 distinct colony morphologies were identified and categorized as Small-colony variant, Spreader and Smooth (Kirisits, Prost et al. 2005, Kirov, Webb et al. 2007, Rau, Hansen et al. 2010, Petrova, Cherny et al. 
2014). Colony variants have been linked in the literature to differences in production of extracellular polysaccharides: Alginate, Pel and Psl developing in different rates depending on environmental conditions (Rakhimova, Munder et al. 2008, Colvin, Gordon et al. 2011, Mowat, Paterson et al. 2011, Colvin, Irie et al. 2012).

Using biofilms treated for 1-3 days, no statistically significant difference in variant ratio was found when comparing dispersed and non-dispersed biofilms. The factor that contributes most to the variance ( $>75 \%)$ between samples is the colistin concentration in the Congo-red plates, unaffected by arabinose addition or sub-optimal colistin treatment. Therefore, under the current experimental conditions, induction of biofilm dispersal combined or not with colistin exposure, at sub-MBEC does not trigger selection of biofilm variants.

Selected specific morphotype variants were compared to the original strain for motility, biofilm production, and MIC parameters. Evidently, selected variants seemed to revert to phenotypes similar to the w.t susceptible population. This was particularly noticeable as the variants were selected on agar containing colistin at $32 \mu \mathrm{g} / \mathrm{mL}$, but their colistin MIC values ranged from 1 to $8 \mu \mathrm{g} / \mathrm{mL}$, following sub-culturing. This phenomenon is again consistent with previous reports on colistin variants in Gram-negative bacteria (Moskowitz, Brannon et al. 2012, Workentine, Sibley et al. 2013, Lee, Na et al. 2014); and correlates with the selection of tolerant or heterogeneous resistant cells present initially in the w.t populations at very small proportions (Zavascki, Carvalhaes et al. 2010, Miller, Brannon et al. 2011, Hermes, Pormann Pitt et al. 2013). 
When examining specific virulence phenotypes, almost all tested colonies tested in the Smooth and SCV morphotypes exhibited increased surface motility behaviors as compared to the w.t susceptible strain. Only the Spreaders demonstrated reduced swimming motility, swarming motility and twitching motility behaviors. These results contrast with the literature, which typically reports biofilm variants, and especially SCV, as populations with decreased or impaired surface motility (Harmsen, Yang et al. 2010, Workentine, Sibley et al. 2013, Davies, James et al. 2017). However, it is important to note that identification of SCV variants is extremely challenging, as their morphology highly depends on growth conditions (type of agar, temperature, duration). Furthermore, a SCV on day 1 could turn into a Smooth or Spreader morphotype after few days at room temperature (results not shown). In contrast, Spreader variants were more readily identifiable as their morphology remains the same on selective plates from day 1 and onwards. Motility behaviors and biofilm forming capacity of these variants were consistent with literature; showing reduced surface motility and increased biofilm production as compared to the w.t.

In the present study, four independent experiments were performed; two of which were used as the pool for colony selection. 20 colonies were evaluated in total by assessing biofilm production and motility behaviors. Experimental conditions for variants identification represent a significant challenge in this type of research. More accurate observations require stringent protocols to be developed, multiple independent experiments to be performed and numerous variants to be tested. Another limitation of this study is the limited number of virulence phenotypes assessed to characterize the variants. Other phenotypic 
assays could have been utilized to better identify the diversification of variants in the population, such as the production of exoproducts (pyoverdine, pyocyanin, rhamnolipids, elastase, proteases) or cell adherence.

Finally, the duration of the experiment may also represent a limitation of this study. A 3-day duration was chosen based on previous findings observed in in-vitro flow cell systems (Chua, Yam et al. 2016). However, selection of mutants or irreversible variants from heterogeneous populations under static conditions may require more extended duration. Due to the fact that no significant change to the distribution of morphotypes was evident in the experiment over the course of four repetitions; it was decided to attempt growing biofilms to the point of 12 days. 10 days are often the minimal duration of time necessary to the development of colistin-resistant PAO1 strains in-vitro, in static and continuous cultures both (Giacometti, Cirioni et al. 1999, Jochumsen, Marvig et al. 2016, Dößelmann, Willmann et al. 2017).

In an attempt to follow colistin evolution using the experimental protocol developed here, a preliminary experiment performed over 7 to 12 days was performed. Significant changes were observed and might be a possible lead; with regards to the emergence of the different morphotypes: After prolonged exposure at $8 \mu \mathrm{g} / \mathrm{mL}$ for 7 days, an increase in the number of the Spreaders was observed at $32 \mu \mathrm{g} / \mathrm{mL}$. Furthermore, an increase in CFU/Bead has been observed at the highest concentrations though even in this scenario, c-di-GMP mediated biofilm dispersal once more, did not affect the ratios between the variants. 
The differences observed at 12 days were the most evident at the highest concentrations tested. Only at that time point samples that have undergone induced biofilm dispersal had a significant difference to undispersed samples, especially appearance of all morphotypes at $64 \mu \mathrm{g} / \mathrm{mL}$. It is within this timeframe that colistin resistance can be evolved in-vitro for planktonic P. aeruginosa (Dößelmann, Willmann et al. 2017). Results suggest that there is potential to evolve biofilm-specific colistin resistant mutants when longer time scales are applied. Unfortunately, due to the complexity of experimental setup and time constraints it was decided to focus on variant generation using the classic technique of serial passages through a gradient of increasing antibiotic concentration (Giacometti, Cirioni et al. 1999).

In conclusion, c-di-GMP mediated biofilm dispersal together with antimicrobial treatment does not increase the risk of development of biofilm-derived variants. These results suggest that c-di-GMP mediated biofilm dispersal could be an interesting strategy at early stages of infections when resistant or tolerant variants have not developed yet as a result of environmental stresses. Further work remains warranted to strengthen the present observations and could include additional experiments with standardized and optimized protocols for identification of biofilm-derived variants. 


\section{Chapter 4: Utilization of Induced c-di-GMP Mediated}

\section{Dispersal as a Strategy to Eradicate Biofilms of}

\section{Colistin Resistant Strains}

\subsection{Introduction}

Evolution of resistance to antimicrobials is an adaptation of the cell population through mutation and selection. The process is temporal by its nature, as environmental conditions change. Naturally, some mutations that cause increased fitness to overcome specific stress will result in lowered fitness to other stress factors. The principle has been initially shown in Gram-positive Staphylococcus species (Sieradzki and Tomasz 1997), where increased resistance to one antibiotic (vancomycin) results in higher susceptibility to other antibiotics such as $\beta$-lactams (Barber, Ireland et al. 2014, Dilworth, Ibrahim et al. 2014). This effect, termed "Antibiotic Seesaw Effect", has frequently been seen as a result of evolved resistance, particularly to the groups of glyco- and lipo-peptides (such as vancomycin and daptomycin, respectively), which target cellular membranes (Vignaroli, Rinaldi et al. 2011, Ortwine, Werth et al. 2013).

Colistin, similar to vancomycin or daptomycin in Gram-positive targeting treatments; is a peptide antibiotic targeting the lipid-A lipopolysaccharide present at the outer cell membranes in Gram-negative pathogens such as P. aeruginosa (Lee, Park et al. 2016). Evolution of colistin resistance in $P$. aeruginosa has been shown to occur through mutations in genes of a plethora of two-component systems, such as phoPQ, pmrAB (Fernández, 
Álvarez-Ortega et al. 2013, Lo Sciuto and Imperi 2018) with high redundancy. Mutations always lead to the upregulation of the enzymatic cascade that is responsible for the 4-amino-arabinose modification of Lipid-A, transcribed by the arnBCADTEF-pmrE operon (Fernández, Gooderham et al. 2010, Dößelmann, Willmann et al. 2017). Through the action of the enzymes which are the products of the operon, the modifications hide the negative charge of Lipid-A and limit binding of polymyxins (Breidenstein, de la Fuente-Núñez et al. 2011, Miller, Brannon et al. 2011, Lo Sciuto and Imperi 2018). The PhoP/Q and PmrA/B two-component systems, are central signal transduction systems, variations in their expression also affect other cellular attributes, such as virulence, motility and metabolism (Rodrigue, Quentin et al. 2000).

Two-component systems comprise of membrane sensor histidine-kinase proteins, $\mathrm{PhoQ}$ and $\mathrm{PmrB}$, which phosphorylate their respective response regulators PhoP and PmrA. The cytoplasmic response regulators, once phosphorylated have an affinity to specific genes and thus modify different cellular response, until dephosphorylated by the kinase protein. The two systems are activated by several signals, most notably, by lower divalent $\mathrm{Mg}^{+2}$ cations in the bacterial periplasm (McPhee, Bains et al. 2006). Colistin replaces $\mathrm{Mg}^{+2}$ that interact with Lipid-A as a necessary step before binding; thus, activation of the kinases correlates to colistin resistance (Biswas, Brunel et al. 2012).

Resistance to colistin, one of the last resort drugs, is increasing globally at an alarming rate (CDC 2017). Transferrable plasmids carrying mcr-1, mcr-2 and most recently mcr-3 genes encoding phosphor-ethanolamine transferase 
enzymes have been reported worldwide (Denton, Kerr et al. 2002). In different bacterial species (Yin, Li et al. 2017), including $P$. aeruginosa (Lee, $\mathrm{Na}$ et al. 2014). Non-heritable and plasmid independent ability to survive prolonged exposure to high concentrations of colistin has also been shown in P. aeruginosa, both in-vitro and in-vivo (Høiby, Krogh Johansen et al. 2001, Moskowitz, Brannon et al. 2012, Dößelmann, Willmann et al. 2017, Regenbogen, Willmann et al. 2017, Tan, Vidaillac et al. 2017). Similarly, biofilms of $P$. aeruginosa show increased tolerance to colistin due to the heterogeneity of the cell population and decreased diffusion of the drug (Harmsen, Yang et al. 2010). Furthermore, specific mutations seem to proliferate in biofilm phase more extensively than in the planktonic phase bacteria (Pamp, Gjermansen et al. 2008). This phenomenon is of particular concern, as it represents a significant therapeutic challenge.

In a recent study (Vidaillac, Benichou et al. 2012), unconventional combinations of antimicrobial agents were evaluated as novel alternatives to treat colistin-resistant infections. It was shown that sub-inhibitory concentrations of colistin combined with vancomycin or trimethoprim resulted in a significant synergistic effect against colistin-resistant isolates of P. aeruginosa, Klebsiella pneumonia (K. pneumonia) and Acinetobacter baumanii ( $A$. baumanii). Vancomycin is a glycopeptide antibiotic that binds to enzymes that catalyze the production of peptidoglycan (Hu, Peng et al. 2016). Vancomycin targets the Lipid II peptidoglycan precursor as it is added to the cell wall, in the periplasmic space. Vancomycin thus has higher efficacy against Gram-positive bacteria, which produce thicker peptidoglycan, hence more targets. In Gram-negative bacteria the existence of the outer membrane 
is thought to be the main cause, perturbing vancomycin's access and its killing effect. Certain E. coli strains with mutations in genes that effect outer membrane assembly have been found to be more susceptible to vancomycin (Zhou, Kang et al. 2015). Although the mechanisms remain unclear, it is speculated, that colistin resistant variants selected from the heterogeneous susceptible w.t population demonstrate increased susceptibility to various antimicrobials, including vancomycin. Following membrane composition changes in resistant populations, it is suspected that they might become susceptible to other antibiotics (Dößelmann, Willmann et al. 2017); in a manner that resembles the "Seesaw Effect". The potential synergy of these combinations in colistin resistant biofilm settings has never been assessed.

Given the promising results observed in the previous chapters, the aims in this section are (1) testing the potential of colistin plus vancomycin combination to treat bacterial biofilms of colistin susceptible and resistant strains, and (2) evaluating the impact of biofilm dispersal on the antimicrobial efficacy of the combination. It is here shown that following induced c-di-GMP mediated dispersal; the seesaw effect is facilitated, enabling a higher synergistic effect between colistin and vancomycin, to eradicate the resistant biofilms. The results demonstrate how the development of resistant bacteria populations could be prevented, and consecutively, once resistant populations develop, the best strategy to eradicate them is based on combinatorial treatment that includes dispersal agents. 


\subsection{Methods}

Bacterial strains: The strain of $P$. aeruginosa used in this section is PAO1/p $\mathrm{p}_{B A D^{-}} y h j H$; a plasmid carrying $y h j H$ under the $\operatorname{araBAD}$ promoter. This strain is susceptible to colistin (MIC 1-2 $\mu \mathrm{g} / \mathrm{mL}$ ). An in-vitro selected colistin resistant derivative of $\mathrm{PAO} 1 / \mathrm{p}_{B A D^{-}} \mathrm{yhjH}$ was generated through repeated exposure to sub-inhibitory concentrations of colistin; evolution of resistance was conducted in batches, in MHB media, with a stepwise increase in colistin concentration (Giacometti, Cirioni et al. 1999, Cabot, Zamorano et al. 2016). Gentamicin at $20 \mu \mathrm{g} / \mathrm{mL}$ was added to the broth to maintain the plasmid, and initial colistin concentration of $2 \mu \mathrm{g} / \mathrm{mL}$ (twice the MIC) was also added to the initial broth. Tubes were grown in $37^{\circ} \mathrm{C}$ with shaking at $200 \mathrm{rpm}$, if following 24 hours, visible turbidity was evident; the sample was used to inoculate a new tube (1:100 dilution) under similar growth conditions at an increased colistin stress. After 14 days of selection, the resistant population was able to grow in $32 \mu \mathrm{g} / \mathrm{mL}$.

Chemical agents: LB agar, Miller recipe (BD Difco) and MHB or MHA, (SigmaAldrich) were used. Antimicrobial agent used for evolution of resistance was colistin-sulfate (MP Biomedicals). Susceptibility was tested versus a total of 25 antibiotic compounds: Colistin sulfate, Polymyxin B sulfate, Ampicillin sodium, Carbenicillin disodium, Neomycin sulfate, Kanamycin sulfate, Streptomycin sulfate, Tetracycline hydrochloride \& Rifampin (MP Biomedicals); Penicillin G sodium, Amoxicillin trihydrate, Piperacillin sodium, Aztreonam, Cefsulodin sodium, Ceftazidime hydrate, Imipenem monohydrate, Meropenem trihydrate, Spectinomycin dihydrochloride pentahydrate, Tobramycin, Vancomycin 
hydrochloride, Nalidixic acid sodium, Ciprofloxacin, Chloramphenicol, Erythromycin \& Sulfadimethoxine (Sigma-Aldrich).

Culture conditions: The parent PAO1/p $\mathrm{p}_{B A D^{-}} y$ hjH strain was routinely cultured onto MHA supplemented with gentamicin sulfate at $60 \mu \mathrm{g} / \mathrm{mL}$. Cultures were freshly streaked on MHA + gentamicin $60 \mu \mathrm{g} / \mathrm{mL}$ plate prior to further experiments.

Biofilm production: Crystal Violet (CV) staining was used to assess biofilm production as previously described (O'Toole 2011, Chua, Hultqvist et al. 2015). Briefly, 24-well plates containing $500 \mu \mathrm{L}$ of fresh MHB broth per well were inoculated with $500 \mu \mathrm{L}$ of the bacterial suspension at $0.01 O^{0 . D_{600}}$. Cultures were left overnight to grow at $37^{\circ} \mathrm{C}$. Biofilms were then washed with $\mathrm{NaCl} 0.9 \%$, stained with $\mathrm{CV}$ solution $\left(0.01 \% \mathrm{vol} / \mathrm{vol}\right.$ in $\left.\mathrm{dH}_{2} \mathrm{O}\right)$ and solubilized in ethanol. Absorbance at $590 \mathrm{~nm}$ was measured using a microplate reader (Infinite Pro 200, Tecan). Experiments were performed in quadruplicates to ensure reproducibility; results are expressed as the average value +/- STDEV $(\mathrm{N}=4)$.

Antimicrobial susceptibility assay: MIC values of parent and resistant strains were assessed in MHB as described in previous chapters using 96-well plate broth dilution method (CLSI 2012). Antimicrobials tested are specified under "Chemical Agents" above at concentrations ranging from 0-128 $\mu \mathrm{g} / \mathrm{mL}$. MIC values were defined as the first concentrations for which the turbidity read at O. $D_{600}$ was not higher than 3 times the control wells, supported by unaided visual inspection. The experiment was performed in triplicates (consisting of 2 
technical duplicates) to ensure reproducibility; Results are expressed as average values +/- STDEV $(\mathrm{N}=3)$.

Growth Curves: Growth kinetics were performed in 24-well plates, containing $500 \mu \mathrm{L}$ of fresh MHB broth, inoculated with $500 \mu \mathrm{L}$ of the samples at $0.01 O^{0 . D_{600}}$. Plates were covered with parafilm to avoid condensation on the lid and incubated at $37^{\circ} \mathrm{C}$ under shaking conditions (200 rpm). Plates were taken out of the incubator at specific intervals (initially every hour, and at shorter intervals at the logarithmic growth phase) to measure turbidity by $O . D_{600}$ with a microplate reader. Experiments were performed in quadruplicates to ensure reproducibility; results are expressed as average turbidity measurement +/- STDEV $(\mathrm{N}=4)$.

Motility Assays: Agar plates were prepared to assess surface motility behaviors as described in the previous chapter. Agar consisted of glucose (1\%), peptone $(0.5 \mathrm{gr} / \mathrm{l})$, yeast $(0.2 \mathrm{gr} / \mathrm{L})$ and agar at concentrations varying from $0.3 \%$ (Swimming) to $0.5 \%$ (swarming), up to $0.9 \%$ (Twitching). Agar plates were inoculated with a sterile toothpick, using single colonies, and incubated at $30^{\circ} \mathrm{C}$. Swimming and swarming plates were imaged directly using the gel imaging station by UV fluorescence and images analyzed with ImageJ software (https://imagej.nih.gov/ij), to calculate the area occupied by bacteria on each plate in $\mathrm{mm}$. Twitching motility was visualized by staining the biofilm with $\mathrm{CV}$, and a ruler was used to measure its diameter (in $\mathrm{mm}$ ). Motility assays were performed in triplicates (consisting of 2 technical replicates) to ensure reproducibility; results are expressed as average +/- STDEV $(\mathrm{N}=3)$. 
Virulence Factors: Production of compounds known to be involved in the virulence of $P$. aeruginosa has been quantified using different biochemical protocols. Inoculums were grown in MHB containing $30 \mu \mathrm{g} / \mathrm{mL}$ gentamycin at $37^{\circ} \mathrm{C}$ in a shaking incubator at $200 \mathrm{rpm}$. After O.N. growth, bacterial suspensions were pelleted, washed in $\mathrm{NaCl} 0.9 \%$ and resuspended in $\mathrm{MHB}$ up to an $O . D_{600}$ of 0.5 . These suspensions were used to inoculate $50 \mathrm{~mL} \mathrm{MHB}$ in long neck Erlenmeyer flasks with metal caps $\left(O . D_{600}\right.$ of 0.01$)$ and left to grow O.N. under the same conditions. Sample turbidity was then measured and corrected to $O . D_{600}=1$ for normalization of the samples. Bacterial suspensions were then pelleted at $10,000 \mathrm{rpm}$ for 10 minutes. The resulting supernatant was aliquoted for the following procedures.

Pyocyanin quantification: Pyocyanin was extracted as described previously (Frank and Demoss 1959) with modifications. $5 \mathrm{~mL}$ samples of the above supernatant were extracted with $1 \mathrm{~mL}$ chloroform. $900 \mu \mathrm{L}$ of the organic phase formed at the bottom of the tubes were pipetted out and transferred into $1.5 \mathrm{~mL}$ Eppendorf tubes. The samples were subsequently centrifuged for 1 minute $10,000 \mathrm{rpm}$ to remove impurities. The organic phase $(700 \mu \mathrm{L})$ was further extracted with $0.1 \mathrm{~N} \mathrm{HCl}$ solution $(700 \mu \mathrm{L})$. The aqueous phase formed at the top was collected for measurement at $520 \mathrm{~nm}$ using a spectrophotometer (UV-Vis, Shimazu). A $0.1 \mathrm{~N} \mathrm{HCl}$ solution was used as a blank. The concentration of Pyocyanine $(\mu \mathrm{g} / \mathrm{mL})$ was estimated by multiplication of $\mathrm{Abs}_{520} \times 17.072$ molar extinction coefficient $\varepsilon$ (El-Shouny, AlBaidani et al. 2011). The experiment was performed in triplicates to ensure reproducibility, and results are expressed as mean +/- STDEV (N=3). 
Rhamnolipid quantification: Difference in rhamnolipid production was determined as described previously (Koch, Käppeli et al. 1991) with additional modifications. Rhamnolipids were extracted from an aliquot of the above supernatant $(20 \mathrm{ml})$ using $5 \mathrm{~mL}$ of diethyl-ether. $4 \mathrm{ml}$ of the organic phase formed at the top were carefully pipetted out using a $5 \mathrm{~mL}$ pipette and transferred into a $15 \mathrm{~mL}$ Falcon tube. Tubes were then centrifuged at $10 \mathrm{k} \mathrm{rpm}$ for 2 minutes to remove any residues of the supernatant. $2 \mathrm{~mL}$ of the transparent top layer were pipetted out and transferred into $2 \mathrm{~mL}$ Eppendorf tubes. Samples were left to evaporate overnight in a chemical hood. Residues were then solubilized in $100 \mu \mathrm{L} \mathrm{dH}_{2} \mathrm{O}$ to which $900 \mu \mathrm{L}$ of a solution of $0.19 \%$ Orcinol in 53\% Sulfuric Acid $\left(\mathrm{H}_{2} \mathrm{SO}_{4}\right)$ were added. Samples (including a blank sample containing the stated solubilization buffer only) were heated for 30 minutes using a heat block at $80^{\circ} \mathrm{C}$. Samples were then cooled down at room temperature for 15 minutes. Absorption was measured using a spectrophotometer at $421 \mathrm{~nm}$. The experiment was performed in triplicates to ensure reproducibility, and results are expressed as mean +/- STDEV (N=3).

Elastase assay: To assess the levels of extracellular proteolytic enzymes, the Congo-red - elastin assay was used as described (Kessler and Safrin 2014). $2 \mathrm{~mL}$ of the above supernatants were filtered through $0.2 \mu \mathrm{m}$ filters. $1 \mathrm{~mL}$ was pipetted out and transferred into $1.5 \mathrm{~mL}$ Eppendorf tubes. Elastin - Congo-red (10 mg) was added to each tube. Samples were vortexed for 30 seconds and incubated at $37^{\circ} \mathrm{C}$ under shaking conditions (200 rpm) for 16 hours. Following incubation, samples were centrifuged down at $14 \mathrm{k} \mathrm{rpm}$ for 1 minute, and absorbance was measured at $495 \mathrm{~nm}$ using a spectrophotometer. A blank sample was prepared and consisted of MHB plus Elastin - Congo-red. 
The experiment was performed triplicates to ensure reproducibility, and results are expressed as mean $+/$ - STDEV $(\mathrm{N}=3)$.

Population Analysis Profile (PAP): The presence of subpopulations with varying degree of susceptibility to colistin was evaluated as previously described ( $\mathrm{Li}$, Rayner et al. 2006). O.N cultures from both strains were centrifuged 8 minutes at $8 \mathrm{~K} \mathrm{rpm}$ and solubilized to high density $\left(\sim 10^{10} \mathrm{CFU} / \mathrm{mL}\right)$ in $\mathrm{MHB}$. Serial dilutions were prepared in $0.9 \% \mathrm{NaCl}$ and $100 \mu \mathrm{L}$ were spread plated on MHA containing $0,1,2,4,8,16,32,64 \mu \mathrm{g} / \mathrm{mL}$ colistin. After 24 -hour incubation at $37^{\circ} \mathrm{C}$, colonies were counted, and $\log _{10} \mathrm{CFU} / \mathrm{mL}$ were calculated and plotted versus concentration. Bacteria on plates with higher colistin concentrations demonstrated slower growth and were therefore allowed to develop for an additional 24 hours at room temperature. The experiment was conducted in quadruplicates $(\mathrm{N}=4)$ to ensure reproducibility. Hetero-resistance was defined as colonies that grew on plates with colistin concentrations higher than the MIC $(1-2 \mu \mathrm{g} / \mathrm{mL})$.

DNA extraction, purification, sequencing and analysis: Samples of resistant and parent strains were grown as described above in "Growth Curves". Cultures were harvested at early stationary phase, where $800 \mu \mathrm{L}$ of each isolate was pelleted down (10k rpm for $5 \mathrm{mins}$ ), and the pellet washed with $0.9 \% \mathrm{NaCl}$ to remove any residual growth media. QIAamp DNA mini Kit (Qiagen) was used for nucleic acid extraction and DNA purification following the manufacturer's protocol. DNA samples were examined on $1 \%$ Agarose Gel in Tris-Acetate-EDTA (TAE) buffer, with a $1 \mathrm{kbp}$ gene ladder (Qiagen). The purity of the DNA was determined by NanoDrop (Thermo-Fischer) and 
accurate concentration by Qubit $^{\circledR} 2.0$ Fluorimeter (Thermo-Fischer) following manufacturer protocols. Whole genome sequencing of all the isolates was carried out using Illumina HiSeq2500 platform generating approximately 20 million, 100 bp paired-end reads.

All further bioinformatics analysis was conducted in CLC Genomics Workbench 10.0 (Qiagen Bioinformatics). Quality and adaptor trimming was performed, and any reads below 50bp were discarded. Paired-end reads were then mapped on the PA01 genome (NCBI Accession Number: NC_002516), and variant detection and comparison was carried out. Any SNPs/ nucleotide differences below the frequency of $90 \%$ were not processed any further.

RNA extraction, purification, sequencing and analysis: Samples of resistant and parent strains were grown in quadruplicates as described above in "Growth Curves". Cultures were harvested at early stationary phase, where $600 \mu \mathrm{L}$ of each isolate $\left(10^{8}-10^{9} \mathrm{CFU} / \mathrm{mL}\right)$ were pelleted down $(7 \mathrm{k} \mathrm{rpm}$ for 5 minutes), and pellets washed with $1.2 \mathrm{~mL}$ of RNA protector, with vortex for 15 seconds. Mixture Incubated for 5 minutes then Pelleted (7k rpm, 12 minutes) at $4^{\circ} \mathrm{C}$. Supernatant completely removed and samples stored at $-80^{\circ} \mathrm{C}$.

RNeasy Mini-Kit (Qiagen), was used for nucleic acid extraction and RNA purification, based on an on-column DNase digestion principal. The purity of the RNA was determined by NanoDrop (Thermo-Fischer) and accurate concentration by Qubit $^{\circledR} 2.0$ Fluorimeter (Thermo-Fischer), Turbo DNA-free treatment (Ambion, Agilent), was used to degrade residual DNA. Agencourt 
RNAClean up XP kit (Beckman-Coulter) magnetic beads were used for RNA clean-up. rRNA depletion performed with RiboZero (Illumina) magnetic beads, RNA integrity was evaluated with Tape-Station (Agilent), samples with excellent RNA Integrity Number $(\mathrm{RIN})>8.0$ were processed further. Doublestranded cDNA was synthesized with Next RNA ultra-directional sequencing (NEB) from depleted samples and purification of double-stranded cDNA with Agencourt AMPure XP beads (Beckman-Coulter). Sequencing of cDNA end-product of all isolates (susceptible and resistant strains in three biological replicates) was carried out using Illumina miSeq platform generating 20 million, 300 bp reads. Gene annotation was done using GO (Gene Ontology) annotation (The Gene Ontology 2015).

All further bioinformatics analysis was conducted in CLC Genomics Workbench 10.0 (Qiagen Bioinformatics): Transcript count (reads above 50 bp in size) calculated based on metadata associated with triplicate samples following normalization to their effective size. Statistics are based on a fit to a generalized linear model with a negative binomial distribution, DESeq2 (Anders and Huber 2010). Differentially expressed genes were identified and were determined as differentially expressed when having an absolute fold change $>2$ and an adjusted $p$-value $<0.05$. Results transformed to $\log _{2}(N+1)$ followed by Heatmap and Principle component analysis (PCA) generation for the differentially expressed genes using d3heatmap and gplot packages in R/Bioconductor (Gentleman, Carey et al. 2004).

Biofilm antibiotic susceptibility assays: Differences in susceptibility between the parent strain ("Susceptible") and the evolved strain ("Resistant") to all 
antibiotic compounds listed above. MBIC and MBEC values were determined using the Calgary Biofilm Pin Lid Device (CBD) (NUNC ${ }^{\text {TM }}$, Thermo-Fischer) against 1-day biofilms grown in MHB, as described in chapter 3 (Ceri, Olson et al. 1999). A growth plate prepared with inoculums at $5 \times 10^{5}-10^{6} \mathrm{CFU} / \mathrm{mL}$ final, placed in the $37^{\circ} \mathrm{C}$ incubator. Following growth phase, the lid is removed and put in a 96-well wash plate containing $200 \mu \mathrm{L} 0.9 \% \mathrm{NaCl}$ in each well. Challenge plates were prepared similarly as MIC, for all 25 compounds (0-128 $\mu \mathrm{g} / \mathrm{mL}$ gradients), lids transferred to challenge plates and incubated at $37^{\circ} \mathrm{C}$ for 24 hours. MBIC was analyzed by both eye and O. $D_{600}$ measurement, then MBEC was determined with resazurin REMA assay as described: CBD moved to an "Assay Plate" containing $200 \mu \mathrm{L}$ MHB media with 60uM final resazurin concentration. Plates were placed in the $37^{\circ} \mathrm{C}$ incubator for 4 hours, then measured in the microplate reader for fluorescence by excitation at $550 \mathrm{~nm}$ and Emission at $600 \mathrm{~nm}$. The experiment was performed in triplicates to ensure reproducibility (consisting of 2 technical replicates); Results are expressed as average values +/- STDEV $(\mathrm{N}=3)$.

Biofilm time-kill kinetics: Antimicrobial efficacy was assessed against 3-day-old biofilms formed on the surface of $5 \mathrm{~mm}$ glass beads, as described in chapter 2. Two beads (representing two technical replicates) were placed into each well of a 24-well microtiter plate (NUNC ${ }^{\mathrm{TM}}$, Thermo-Fischer). Biofilms were next exposed to fresh MHB containing $1 \%(\mathrm{w} / \mathrm{v})$ arabinose or arabinose free medium (control samples). After 4 hours, samples were treated with colistin at $8 \mu \mathrm{g} / \mathrm{mL}$ and/or Vancomycin at $64 \mu \mathrm{g} / \mathrm{mL}$. Beads were collected at indicated intervals, subjected to a series of 10 -second sonication (at $37 \mathrm{kHz}$ ) and 10-second vortex. Bacterial suspensions were subsequently serially diluted in 
$0.9 \% \mathrm{NaCl}$ before being drop-plated onto LB agar plates. After 24-hour incubation at $37^{\circ} \mathrm{C}$, the residual biofilm was quantified as colony forming units $\mathrm{CFU} /$ beads and plotted against time. Experiments were conducted in quadruplicates ( 2 beads per well $\times 2$ wells per condition). MDK 99 was defined as the duration of time necessary to eradicate $99 \%$ of the population, relative to controls (Brauner, Fridman et al. 2016). Results are presented as an average CFU/bead from 3 individual experiments (conducted in 2 technical replicates) +/- STDEV.

Confocal Microscopy: The difference in eDNA production between strains was examined using confocal laser scanning microscopy (CLSM, Zeiss 780, Germany). Biofilm samples of both strains were grown on ibidi ${ }^{\circledR} 8$-well glass bottom $\mu$-slides for 3 days before exposure to colistin at $8 \mu \mathrm{g} / \mathrm{mL}$ alone or following 4 hours induced dispersal. After 24 hours antibiotic challenge, samples were stained for 30 minutes, using Syto-9 (Live cell stain) at $3.34 \mu \mathrm{M}$ and DAPI (Total DNA stain) at $1.44 \mu \mathrm{M}$ final concentrations. CLSM further assessed biofilms, Syto-9 (Green channel) excitation with 488-laser line and emission at 499-588 $\mathrm{nm}$ and DAPI (Blue channel) excitation with 405-laser line and emission at 410-502 nm. Five images were acquired in Z-stacks for each sample; the experiment was conducted in triplicates $(\mathrm{N}=3)$. Image sets were subsequently analyzed for biovolume $\left(\mu \mathrm{m}^{3} / \mu \mathrm{m}^{2}\right)$ using COMSTAT 2 ImageJ plugin (Heydorn, Nielsen et al. 2000) using software auto-thresholding.

Statistical analysis: Data analysis, graph plotting and statistical significance (using student's t-test, multiple t-tests, multiple comparisons one-way ANOVA 
\& two-way ANOVA, as indicated in figure captions) were conducted in Prism GraphPad 7.

\subsection{Results}

Phenotypic characterization of colistin susceptible (parent strain) and resistant $\underline{\mathrm{PAO} 1 / \mathrm{p}_{B A D}-\underline{y} \boldsymbol{j} \mathrm{H} H \text { derivatives }}$

Significant phenotypic differences were observed between the colistin resistant derivative of $\mathrm{PAO} 1 / \mathrm{p}_{B A D^{-}} y$ hjH and its susceptible parent strain. Figure 4.1 illustrates the difference in morphology in both agar and liquid medium. The resistant strain produces more pigment and has a SCV phenotype. Furthermore, there were evident aggregates forming in the tubes, regardless of rigorous shaking at $200 \mathrm{rpm}$. This phenotype was evident to a varying extent throughout the evolution process.
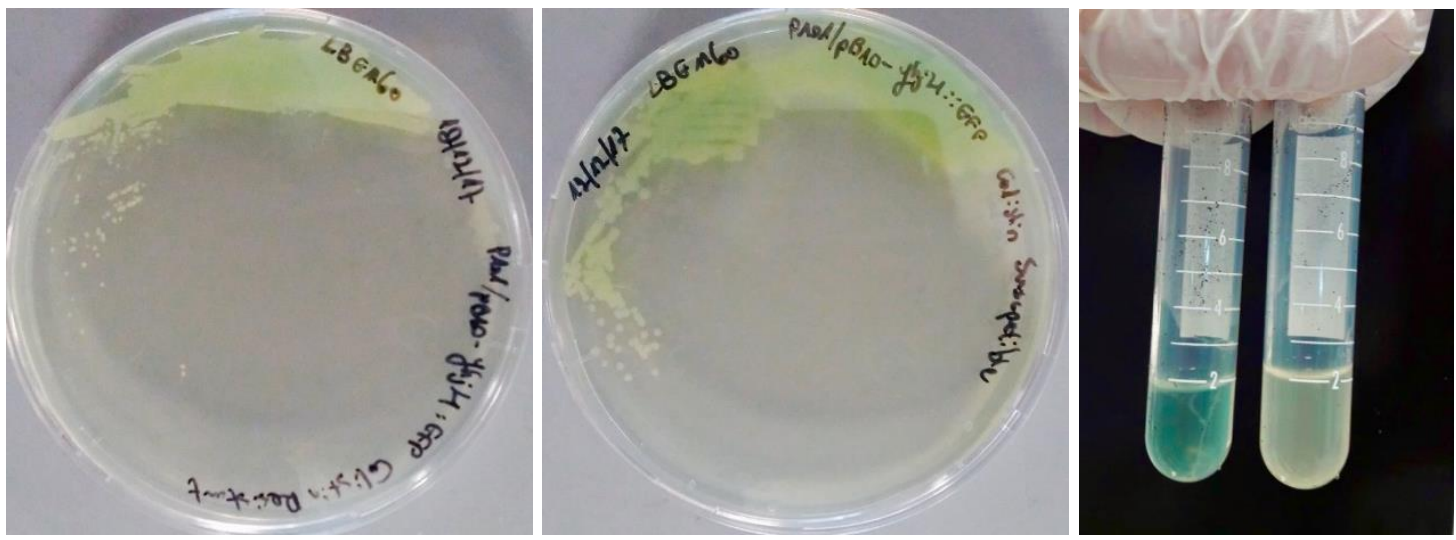

Figure 4.1 Differences in growth patterns between colistin resistant P.aeruginosa PAO1/p $\mathrm{p}_{B A D}-y$ hjH evolved strain (Left) and its parent strain (Middle) on MHA + gentamycin $60 \mu \mathrm{g} / \mathrm{mL}$ plates and in $\mathrm{MHB}+$ gentamycin $30 \mu \mathrm{g} / \mathrm{mL}$ (Right) after overnight growth in $37^{\circ} \mathrm{C}$. 
Both strains exhibited different growth kinetics, (Figure 4.2); when starting from a similar initial inoculum at $O . D_{600}$ at 0.05 , the resistant strain remained in lag phase for approximately 2 hours as compared to 1 hour for the susceptible parent strain. Furthermore, the maximal growth in turbidity was lower for the resistant derivative as compared to its parent strain.

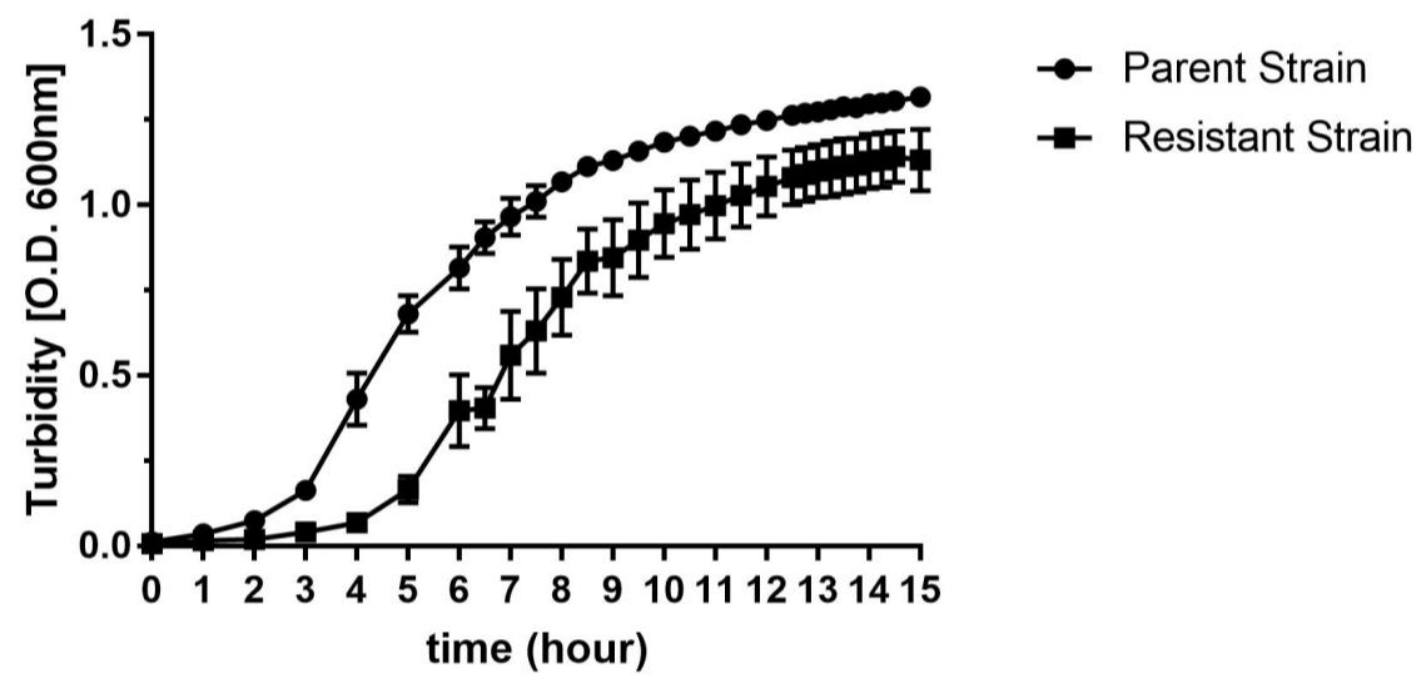

Figure 4.2 The two strains exhibit different growth kinetics, when grown in MHB media at $37^{\circ} \mathrm{C}$ with shaking at $200 \mathrm{rpm}$. Growth was measured as an increase in turbidity at $O . D_{600}$ in 24-well plates; experiments have been conducted in quadruplicates $(\mathrm{N}=3)$.

Striking differences in terms of surface motility behavior were observed between the two related strains (Figure 4.3). The resistant strain was swarming and swimming deficient as compared to its parental strain. 100-fold decrease in swimming and 10-fold decrease in swarming motilities could be identified. 

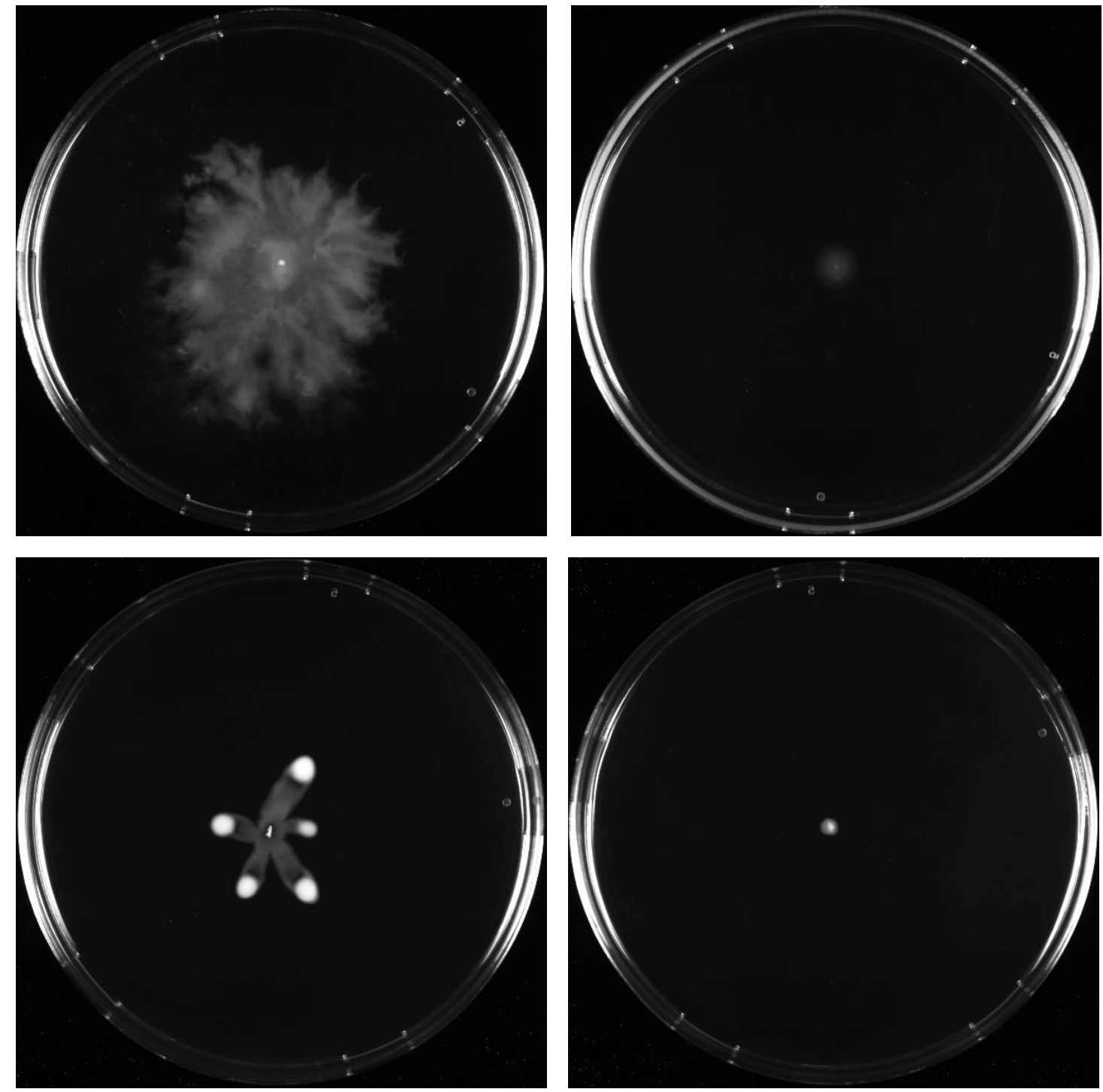

Figure 4.3 Differences in swimming (Top) and swarming (Bottom) motility phenotypes between the PAO1/p $\mathrm{P}_{B A D}-y h j H$ parent (left) and resistant strain (right).

Results for all phenotypic assays are summarized in table 4.1. Assessment of production of a number of known virulence factors shows that the evolved strain produced between 3-4 times more pyocyanin $(2.37+/-0.49 \mu \mathrm{g} / \mathrm{mL}$ versus $0.61+/-0.19 \mu \mathrm{g} / \mathrm{mL}$, for the resistant and parent strains, respectively). 2-3 times more proteolytic enzymes $\left(0.69+/-0.02\right.$ versus $0.24+/-0.01$ Abs $_{495}$ 
in the elastase essay), and also a notable increase in rhamnolipid production $\left(1.16+/-0.06\right.$ versus $0.77+/-0.09$ Abs $\left._{421}\right)$.

Table 4.1 Phenotypic differences between parent and colistin resistant derivative PAO1/p BAD- $y$ hjH strains.

\begin{tabular}{|c|c|c|c|}
\hline Phenotype & Strain & Parent & Colistin Resistant \\
\hline $\begin{array}{c}\text { Biofilm } \\
\text { Production }\end{array}$ & $\mathrm{CV}\left[\mathrm{Abs}_{590 \mathrm{~nm}}\right]$ & $1.015(+/-0.067)$ & $1.013(+/-0.051)$ \\
\hline \multirow{3}{*}{ Motility } & Swimming $\left[\mathrm{mm}^{2}\right]$ & $681.1(+/-80.1)$ & $5.25(+/-0.94)$ \\
\hline & Swarming [mm²] & $35.91(+/-8.97)$ & $1.89(+/-0.99)$ \\
\hline & Twitching [mm] & $7(+/-1)$ & $3(+/-1)$ \\
\hline \multirow{3}{*}{$\begin{array}{l}\text { Virulence Factors } \\
\text { Production }\end{array}$} & Pyocyanin $[\mu \mathrm{g} / \mathrm{mL}]$ & $0.609(+/-0.19)$ & $2.373(+/-0.495)$ \\
\hline & Rhamnolipid $\left[\mathrm{Abs}_{421 \mathrm{~nm}}\right]$ & $0.770(+/-0.086)$ & $1.158(+/-0.058)$ \\
\hline & Elastase $\left[\mathrm{Abs}_{495 \mathrm{~nm}}\right]$ & $0.238(+/-0.004)$ & $0.686(+/-0.023)$ \\
\hline
\end{tabular}

The susceptibilities of the evolved strain versus the parent strain, to various groups of antibiotics, were examined. First, in planktonic phase (MIC) as table 4.2 recapitulates the results. The evolved resistant strain exhibited 3 two-fold decrease in susceptibility to colistin (MIC of 16 to $32 \mu \mathrm{g} / \mathrm{mL}$ versus $1-2 \mu \mathrm{g} / \mathrm{mL}$ for the parent strain). Mutations and adaptations following the evolution of colistin resistance are not exclusive to colistin alone, but allow resistance to other polymyxins (Polymyxin-B). The resistant strain became more susceptible to all $\beta$-lactam antibiotic groups (Narrow \& extended spectrum, cephalosporin \& carbapenem) in varying degrees (4-7 two-fold) virtually restoring all MICs to physiologically relevant concentrations. The effect is less evident in aminoglycosides except for tobramycin (3 two-fold reduction in MIC). A few 
other notable antibiotics that exhibit similar trends are vancomycin and tetracycline (3 two-fold reduction) and rifampicin (4 two-fold reduction).

Table 4.2 MIC values for the PAO1/ $\mathrm{p}_{B A D}-y h j H$ parent and colistin resistant strains.

\begin{tabular}{|c|c|c|c|}
\hline Family & Antibiotic & $\begin{array}{c}\text { Parent strain MIC } \\
(\mu \mathrm{g} / \mathrm{mL})\end{array}$ & $\begin{array}{c}\text { Colistin Resistant } \\
\text { strain MIC }(\mu \mathrm{g} / \mathrm{mL})\end{array}$ \\
\hline \multirow[t]{2}{*}{ Polymyxin } & Colistin (Polymyxin E) & $1-2$ & $16-32$ \\
\hline & Polymyxin B & $1-2$ & $8-16$ \\
\hline $\begin{array}{l}\text { Narrow Spectrum } \\
\beta \text {-lactam }\end{array}$ & Penicillin & $>128$ & $16-32$ \\
\hline \multirow{4}{*}{$\begin{array}{l}\text { Extended Spectrum } \\
\beta \text {-lactam }\end{array}$} & Amoxicillin & $>128$ & $2-4$ \\
\hline & Ampicillin & $>128$ & $4-8$ \\
\hline & Carbenicillin & $16-32$ & $1-2$ \\
\hline & Piperacillin & $8-16$ & $0.5-1$ \\
\hline Monobactam & Aztreonam & $2-4$ & $0.125-0.25$ \\
\hline \multirow[t]{2}{*}{ Cephalosporin } & Ceftsulodin & $0.5-1$ & $<0.125$ \\
\hline & Ceftazidime & $2-4$ & $0.125-0.25$ \\
\hline \multirow[t]{2}{*}{ Carbapenem } & Imipenem & 0.5-1 & $0.25-0.5$ \\
\hline & Meropenem & $0.25-0.5$ & $<0.125$ \\
\hline \multirow[t]{5}{*}{ Aminoglycoside } & Tobramycin & $2-4$ & $0.25-0.5$ \\
\hline & Neomycin & 8-16 & $4-8$ \\
\hline & Kanamycin & $>128$ & $64-128$ \\
\hline & Streptomycin & $>128$ & $>128$ \\
\hline & Spectinomycin & $>128$ & $>128$ \\
\hline Glycopeptide & Vancomycin & $>128$ & $16-32$ \\
\hline \multirow[t]{2}{*}{ Fluoroquinolone } & Nalidixic Acid & $16-32$ & $16-32$ \\
\hline & Ciprofloxacin & $<0.125$ & $<0.125$ \\
\hline Naphthacene & Tetracycline & $16-32$ & $2-4$ \\
\hline Amphenicol & Chloramphenicol & $>128$ & $32-64$ \\
\hline Rifampicin & Rifampicin & $8-16$ & $0.5-1$ \\
\hline Macrolide & Erythromycin & $32-64$ & $32-64$ \\
\hline Sulfonamide & Sulfadimethoxine & $>128$ & $64-128$ \\
\hline
\end{tabular}

The population analysis profile (PAP) (Figure 4.4) of both parent and resistant strains demonstrated heterogeneous resistance to colistin. The susceptible parent strain had a $\sim 1 \times 10^{-6} \%$ detectable sub-population with the ability to survive colistin concentrations of up to $16-32 \mu \mathrm{g} / \mathrm{mL}\left(10.67+/-0.11 \log _{10}\right.$ CFU/Bead for control, versus 3.08-2.88 +/- 0.25, accordingly). The resistant strain exhibited similar PAP pattern as the parent strain, but the resulting curve 
was shifted towards increased concentrations of colistin, with $\sim 1-0.33 \%$ of the population capable of growing at $16-32 \mu \mathrm{g} / \mathrm{mL}\left(9.99+/-0.22 \log _{10}\right.$ CFU/Bead for control, versus 8.02-7.52+/- 0.4, respectively).

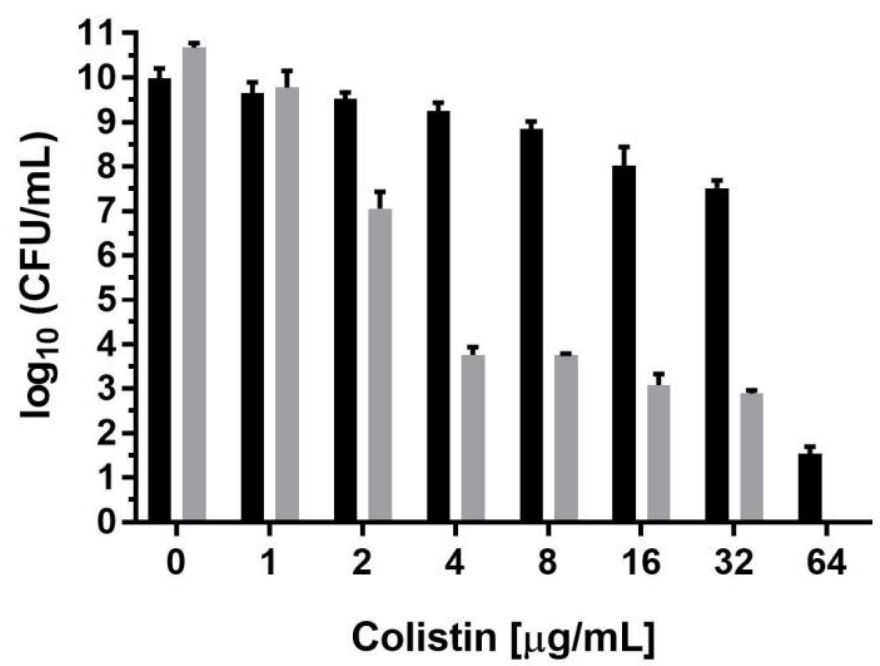

- Resistant Strain

Parent Strain

Figure 4.4 PAP of parent $\mathrm{PAO} 1 / \mathrm{p}_{B A D^{-}} \mathrm{yhjH}$ strain and its colistin resistant derivative. O.N. culture and serial dilutions plated on MHA containing $0-64 \mu \mathrm{g} / \mathrm{mL}$ colistin. Results calculated as $\log _{10}(\mathrm{CFU} / \mathrm{mL})$. The experiment was conducted in triplicates plotted as average values + - STDEV $(\mathrm{N}=3)$.

\section{Genetic characterization of the colistin resistant derivative}

DNA from both $\mathrm{PAO} 1 / \mathrm{p}_{B A D}-y h j H$ strains was extracted and following whole genome sequencing aligned the two genomes against the PAO1 reference genome in the database. A pairwise comparison of the evolved strain's genome versus the parent yielded a significant number of hits indicating a list of point mutations, insertions and deletions. A list of mutations with a $100 \%$ frequency is summed up in table $\mathbf{4 . 3}$ with references to literature. Genes encoding TbpA, PhoQ and LpxC demonstrated significant alterations for a total of 6 mutations. Single-nucleotide Variations (SNV) that resulted in the 
replacement of a purine $(\mathrm{G})$ by a pyrimidine $(\mathrm{T})$ was observed for both phoQ and IpxC. In all cases, variations resulted in an amino acid change in coding regions. For $\mathrm{PhoQ}$, the mutation led to a probable truncated protein product (nonsense). The gene tpbA accumulated the most substantial number of variations as compared to the parent strain. The hyper-mutable hypothetical Pf1 Phage coat protein (Whiteley, Bangera et al. 2001), PA0727, was shown to accumulate the total highest number of 21 mutations, 8 of which in over $90 \%$ frequency $(91.7-99.9 \%)$ in the resistant strain as compared to the parent (Supplementary Table 1).

Table 4.3 Top hits from the pairwise comparison, detected variations between the two $\mathrm{PAO} 1 / \mathrm{p}_{B A D}-y h j H$ strains at $100 \%$ frequency.

\begin{tabular}{|c|c|c|c|c|c|c|c|c|}
\hline \# & $\begin{array}{c}\text { Locus } \\
\text { Tag }\end{array}$ & Gene Name & $\begin{array}{l}\text { Genomic } \\
\text { Location }\end{array}$ & $\begin{array}{c}\text { Mutation } \\
\text { Type }\end{array}$ & Position & Mutation & $\begin{array}{l}\text { A. Acid } \\
\text { Change }\end{array}$ & Reference \\
\hline 1 & $\begin{array}{l}\text { PA } \\
1180\end{array}$ & $\begin{array}{l}\text { PhoQ - Two } \\
\text { Component } \\
\text { Sensor }\end{array}$ & $\begin{array}{c}1278362 \\
- \\
1279708 \\
(+) \\
\end{array}$ & SNV & 1279664 & $\mathrm{G} \rightarrow \mathrm{T}$ & $\begin{array}{l}\mathrm{Glu}_{435}{ }^{*} \\
\text { Nonsense }\end{array}$ & $\begin{array}{l}\text { Jochumsen, } \\
\text { et al. } 2016 \\
\text { Miller, et al. } \\
2011\end{array}$ \\
\hline \multirow{4}{*}{2} & \multirow{4}{*}{$\begin{array}{l}\text { PA } \\
3885\end{array}$} & \multirow{4}{*}{$\begin{array}{l}\text { TpbA - } \\
\text { protein } \\
\text { tyrosine } \\
\text { phosphatase }\end{array}$} & \multirow{4}{*}{$\begin{array}{c}4350834 \\
- \\
4351490 \\
(+)\end{array}$} & SNV & 4351381 & $\mathrm{C} \rightarrow \mathrm{G}$ & $\mathrm{Ala}_{183} \mathrm{Gly}$ & \multirow{4}{*}{$\begin{array}{l}\text { Nicastro, et } \\
\text { al. } 2014 \\
\text { Ueda, et al. } \\
2009\end{array}$} \\
\hline & & & & SNV & 4351393 & $\mathrm{G} \rightarrow \mathrm{A}$ & Gly $_{187}$ Asp & \\
\hline & & & & SNV & 4351395 & $C \rightarrow A$ & Leu $_{188}$ Met & \\
\hline & & & & MNV & $\begin{array}{r}4351387 . \\
.4351388 \\
\end{array}$ & $\mathrm{TC} \rightarrow \mathrm{AG}$ & Val $_{185} \mathrm{Glu}$ & \\
\hline 3 & $\begin{array}{l}\text { PA } \\
4406\end{array}$ & $\begin{array}{l}\text { LpxC - UDP- } \\
\text { 3-O-acyl-N- } \\
\text { acetyl- } \\
\text { glucosamine } \\
\text { deacetylase }\end{array}$ & $\begin{array}{c}4938276 \\
- \\
4939187 \\
(-)\end{array}$ & SNV & 4938732 & $\mathrm{G} \rightarrow \mathrm{T}$ & Phe $_{152}$ Leu & $\begin{array}{l}\text { Grégoire, et } \\
\text { al. } 2017 \\
\text { Boyce, et al. } \\
2014\end{array}$ \\
\hline
\end{tabular}

SNV - Single-nucleotide Variation, MNV - Multi-nucleutide Variation

Gene Expression results:

Extracted RNA from both PAO1/p $\mathrm{p}_{B A D}-y h j H$ strains was used as a template for cDNA sequencing and following filtering, genes were annotated using the GO database. Read counts of all transcriptome triplicates for the susceptible 
parent and resistant strains were then performed. Analysis representing the differences in gene expression between replicates is presented in figure 4.5.

PCA (Figure 4.5A) suggests that $\sim 70 \%$ of variance exists in the differential expression results between strains (PCA1), and 13\% variance exists between replicates (PCA2). The PCA2 dimension analysis suggested that replicates of the resistant strain were at most twice as varied as the susceptible parent strain. The different levels of expression between strains were evaluated by a negative binomial analysis (Figure 4.5B) and was composed of 287 genes satisfying threshold criteria were considered for further analysis, of which, 187 genes were upregulated, and 110 downregulated (Figure 4.5C; Supplementary Tables 2-3, respectively) in the resistant strain as compared to the susceptible parent. 


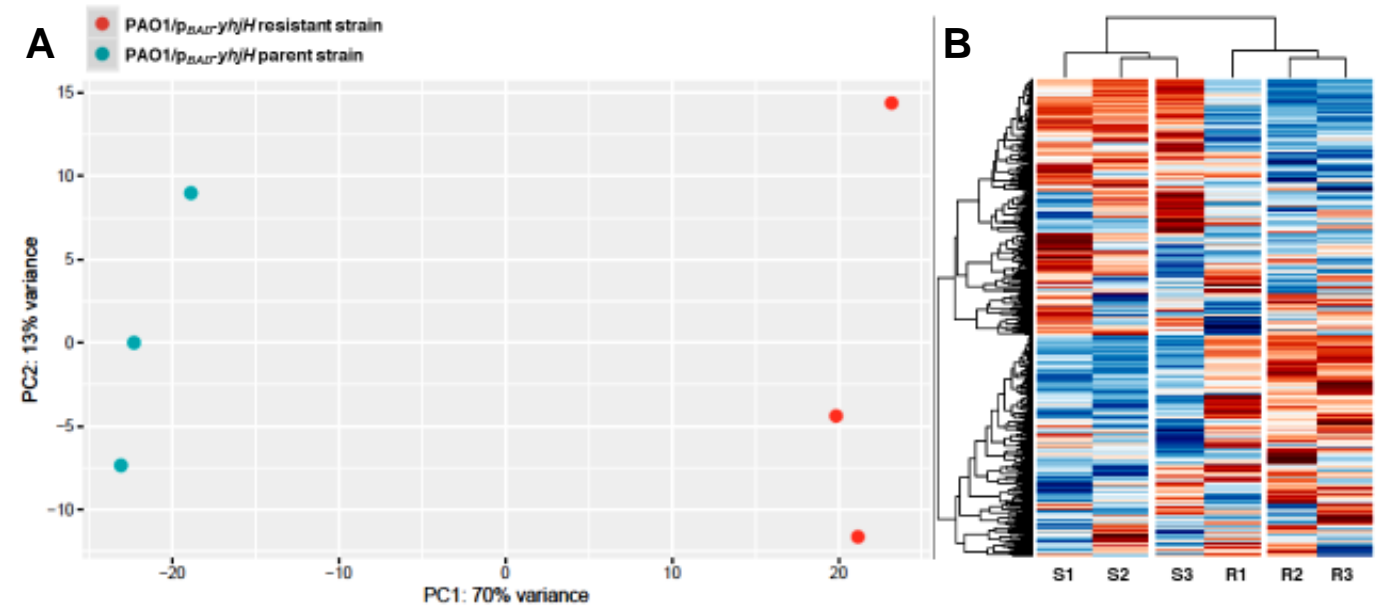

C

Upregulated

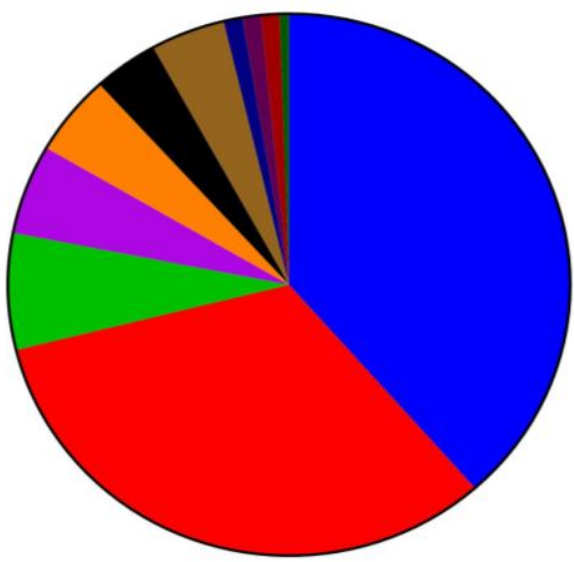

- $38.50 \%$ Hypothetical, Putative, Unknown

- $32.62 \%$ Membrane Proteins \& Transport

- $6.95 \%$ Adaptation / Protection / Antibiotic Resistance

- $5.35 \%$ Amino acid biosynthesis \& metabolism

= $4.81 \%$ Cell wall / LPS / capsule

- $3.74 \%$ Carbon Catabolism

- $1.07 \%$ DNA replication, recombination, modification \& repair

= $4.28 \%$ Transcription Factors

- $1.07 \%$ Fatty acid \& phospholipid metabolism

- $1.07 \%$ Motility / Attachment

- $0.53 \%$ Secreted Factors

Total $=187$

Downregulated



- $31.82 \%$ Hypothetical, Putative, Unknown

- $32.73 \%$ Membrane Proteins \& Transport

- $7.27 \%$ Adaptation / Protection / Antibiotic Resistance

- $5.45 \%$ Amino acid biosynthesis \& metabolism

= $0.91 \%$ Cell wall / LPS / capsule

- $6.36 \%$ Carbon Catabolism

- $10.00 \%$ Transcription Factors

- $1.82 \%$ DNA replication, recombination, modification \& repair

- $1.82 \%$ Motility / Attachment

- $1.82 \%$ Secreted Factors

Total $=\mathbf{1 1 0}$

Figure 4.5 Analysis of RNA sequencing data, of 280 differently expressed genes exhibiting a $>2$ absolute fold-change (adjusted $p$-value $<0.05$ ) generating $(\mathbf{A})$ principal component analysis (PCA) of triplicates of each strain, parent and colistin resistant and (B) expression heatmap in each strain (Red, upregulation; blue, downregulation), (C) pie chart of gene ontology (GO) categorized by biological function (PseudoCAP). 
The gene expressing the highest $\log _{2}$ fold-change (8.27) is PA3559 pmrE, which is part of the arnBCADTEF-pmrE operon (Fernández, Álvarez-Ortega et al. 2013, Olaitan, Morand et al. 2014). 9 additional genes in the operon were detected in similarly high expression levels ( $\log _{2}$ fold-change of 8.21-6.59). The three constituents of the oprH-phoP/Q, which take part in the two-component system that controls the expression of the arn operon were the next to be detected in high levels ( $\log _{2}$ fold-change of 6.24-5.26). The hypothetical proteins PA4517, PA4010, PA4011 and were also upregulated ( $\log _{2}$ fold-change $4.65,4.19,3.89$, respectively), known to be under the control of phoPQ and pmrAB (McPhee, Bains et al. 2006) and related to reduced divalent cation concentrations near bacterial membranes. Mutations in tpbA culminated to higher expression of the gene as well ( $\log _{2}$ fold-change 4.65$)$.

The genes PA3441-7, who were shown in related Pseudomonas species to be part of the cell sulfur transport mechanism (sus operon) (Scott, Hilton et al. 2007) were the top downregulated genes in this analysis ( $\log _{2}$ fold-change $-3.63,-2.75,-2.89,-1.28,-2.25,-1.78$ respectively). The gene tauD, taurine dioxygenase, which is regulated by sus was also found downregulated (-2.29). Generally, different systems related to catabolism of different compounds were the main to be downregulated.

\section{Biofilm characterization}

Differences in antimicrobial susceptibility between biofilms of the 2 strains were assessed using the $\mathrm{CBD}$, as described above. MBIC and MBEC values for 1-day-old biofilms are shown in table 4.4. 
Table 4.4 MBIC and MBEC range values for both $\mathrm{PAO} 1 / \mathrm{p}_{B A D}-y$ hjH strains, 1-day grown biofilms.

\begin{tabular}{|c|c|c|c|c|c|}
\hline \multirow[t]{2}{*}{ Family } & \multirow[t]{2}{*}{ Antibiotic } & \multicolumn{2}{|c|}{ Parent Strain } & \multicolumn{2}{|c|}{ Colistin Resistant Strain } \\
\hline & & MBIC $(\mu \mathrm{g} / \mathrm{mL})$ & $\operatorname{MBEC}(\mu \mathrm{g} / \mathrm{mL})$ & $\mathrm{MBIC}(\mu \mathrm{g} / \mathrm{mL})$ & MBEC $(\mu \mathrm{g} / \mathrm{mL})$ \\
\hline \multirow[t]{2}{*}{ Polymyxin } & Colistin (Polymyxin E) & $4-8$ & $8-16$ & $16-32$ & $32-64$ \\
\hline & Polymyxin B & $8-16$ & $8-16$ & $8-16$ & $8-16$ \\
\hline $\begin{array}{l}\text { Narrow Spectrum } \\
\beta \text {-lactam }\end{array}$ & Penicillin & $>128$ & $>128$ & $>128$ & $>128$ \\
\hline \multirow{4}{*}{$\begin{array}{l}\text { Extended } \\
\text { spectrum } \\
\beta \text {-lactam }\end{array}$} & Amoxicillin & $>128$ & $>128$ & $64-128$ & $64-128$ \\
\hline & Ampicillin & $>128$ & $>128$ & $64-128$ & $64-128$ \\
\hline & Carbenicillin & $8-16$ & $>32$ & $8-16$ & $16-32$ \\
\hline & Piperacillin & $64-128$ & $64-128$ & $8-16$ & $16-32$ \\
\hline Monobactam & Aztreonam & $>128$ & $>128$ & $>128$ & $>128$ \\
\hline \multirow[t]{2}{*}{ Cephalosporin } & Ceftsulodin & $>32$ & $>32$ & $>32$ & $>32$ \\
\hline & Ceftazidime & $>32$ & $>32$ & $>32$ & $>32$ \\
\hline \multirow[t]{2}{*}{ Carbapenem } & Imipenem & $2-4$ & $4-8$ & $0.5-1$ & $0.5-1$ \\
\hline & Meropenem & $2-4$ & $4-8$ & $0.5-1$ & $0.5-1$ \\
\hline \multirow[t]{5}{*}{ Aminoglycoside } & Tobramycin & $0.25-0.5$ & $8-16$ & $1-2$ & $2-4$ \\
\hline & Neomycin & $16-32$ & $32-64$ & $16-32$ & $16-32$ \\
\hline & Kanamycin & $>128$ & $>128$ & $>128$ & $>128$ \\
\hline & Streptomycin & $>128$ & $>128$ & $>128$ & $>128$ \\
\hline & Spectinomycin & $>128$ & $>128$ & $>128$ & $>128$ \\
\hline Glycopeptide & Vancomycin & $>128$ & $>128$ & $64-128$ & $>128$ \\
\hline \multirow[t]{2}{*}{ Fluoroquinolone } & Nalidixic Acid & $32-64$ & $32-64$ & $32-64$ & $32-64$ \\
\hline & Ciprofloxacin & $1-2$ & $1-2$ & $0.5-1$ & $1-2$ \\
\hline Naphthacene & Tetracycline & $32-64$ & $32-64$ & $16-32$ & $32-64$ \\
\hline Amphenicol & Chloramphenicol & $>128$ & $>128$ & $>128$ & $>128$ \\
\hline Rifampicin & Rifampicin & $16-32$ & $16-32$ & $4-8$ & $16-32$ \\
\hline Macrolide & Erythromycin & $>128$ & $>128$ & $64-128$ & $64-128$ \\
\hline Sulfonamide & Sulfadimethoxine & $>128$ & $>128$ & $>128$ & $>128$ \\
\hline
\end{tabular}

Differences in susceptibility to colistin were noticed with MBIC and MBEC values of $4-8$ and $8-16 \mu \mathrm{g} / \mathrm{mL}$, respectively for the parent strain versus $16-32$ and $32-64 \mu \mathrm{g} / \mathrm{mL}$ for the resistant strain. It is noticeable that the same mechanisms that can be attributed to resistance development in planktonic cultures are not the only effectors when evaluating $P$. aeruginosa biofilms. There were no striking differences in MBIC and MBEC values between the parent and resistant strains for most antibiotics, excluding a few. A consistent drop in resistance for both carbapenems (imipenem and meropenem) from 
MBIC of $2-4 \mu \mathrm{g} / \mathrm{mL}$ and MBEC of $4-8 \mu \mathrm{g} / \mathrm{mL}$ for the parent strain to MBIC and MBEC of $0.5-1 \mu \mathrm{g} / \mathrm{mL}$ for the resistant strain. For tobramycin, from the MBIC value of $0.25-0.5 \mu \mathrm{g} / \mathrm{mL}$ for the parent strain, the resistant strain has become less susceptible, up to $1-2 \mu \mathrm{g} / \mathrm{mL}$. However, the MBEC for the resistant strain has decreased, from $8-16 \mu \mathrm{g} / \mathrm{mL}$ for the susceptible to $2-4 \mu \mathrm{g} / \mathrm{mL}$. These results support the results in chapter 2: Imipenem and tobramycin exhibit high efficacy potential in mono-treatment against young biofilms. Rifampicin and piperacillin exhibit 3 two-fold drops in susceptibility: the parent strain shows MBIC and MBEC values for rifampicin at $16-32 \mu \mathrm{g} / \mathrm{mL}$, and the resistant strain shows a similar MBEC, but a drop to $4-8 \mu \mathrm{g} / \mathrm{mL}$ in MBIC is evident. Piperacillin shows a drop from 64-128 $\mu \mathrm{g} / \mathrm{mL}$ MBIC and MBEC values for the parent to 8-16 and 16-32 $\mu \mathrm{g} / \mathrm{mL} \mathrm{MBIC}$ and MBEC values for the resistant.

\section{Difference in EPS eDNA production between parent and resistant strains}

$\mathrm{TpbA}$ is a sensor phosphatase protein negatively regulating the DGC TpbB (Pu and Wood 2010), loss of TpbA has been shown to cascade in increased c-di-GMP production, through TpbB, leading to increased EPS formation (Ueda and Wood 2009, Harmsen, Yang et al. 2010); and to a decrease in eDNA production (Ueda and Wood 2010). Mutations in TpbA were shown to result in both reduced eDNA content, and higher intracellular c-di-GMP. Because the tpbA gene accumulated the highest number of mutations in the resistant strain (Table 4.3), it was hypothesized that it would lead to a decrease in eDNA production, by increasing c-di-GMP.

The roles of eDNA in colistin resistance are thought to be double: As a chelator for both the drug and $\mathrm{Mg}^{+2}$, reducing colistin diffusivity and activity but 
also inducing PhoP/Q (Mulcahy, Charron-Mazenod et al. 2008). To further understand the eDNA production differences between parent and resistant strains CLSM was utilized on 3-day grown biofilm bacteria of both strains. It was hypothesized that induced dispersal by $y h j H$, while reducing intercellular c-di-GMP, would also increase eDNA/Bacteria ratios.

The combined effect of $1 \%$ arabinose induction with $8 \mu \mathrm{g} / \mathrm{mL}$ colistin was further evaluated. Biofilms were stained with two dyes: Syto9 (Green) that stains "live cells", and DAPI, which stains all DNA in the sample. The ratios of DAPI to Syto9 fluorescence were calculated for each treatment of each strain (Figure 4.6). No difference was observed in the control samples (Control Ara-) between the resistant and parent strains (dead/live ratio: $1.49+/-1.33$ versus 1.92 +/- 1.44 DAPI/Syto9, respectively) (Figure 4.6B). Following arabinose induction, a decrease in DAPI/Syto9 was observed in the resistant strain treated with colistin as compared to the untreated control $(0.53+/-0.41$ versus $1.49+/-1.33, p<0.05)$. Two-way ANOVA analysis defined the difference between strains as the most significant independent variable contributing to variance in DAPI/Syto9 ratios $(\sim 12 \%, \mathrm{p}<0.0001)$. Assuming higher intracellular c-di-GMP facilitates lower colistin susceptibility in the resistant strain; arabinose induction of c-di-GMP mediated dispersal is thought to revert this effect, increasing colistin susceptibility. 
A
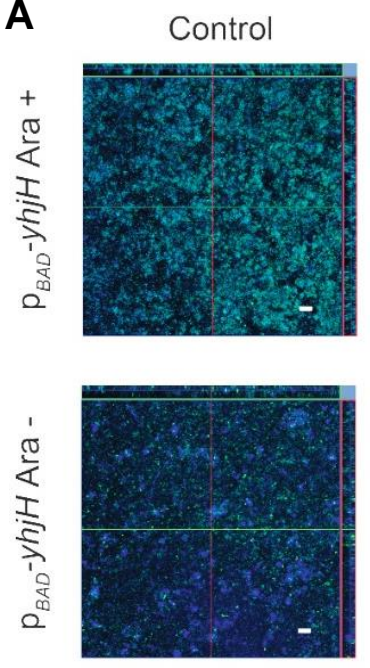

Control
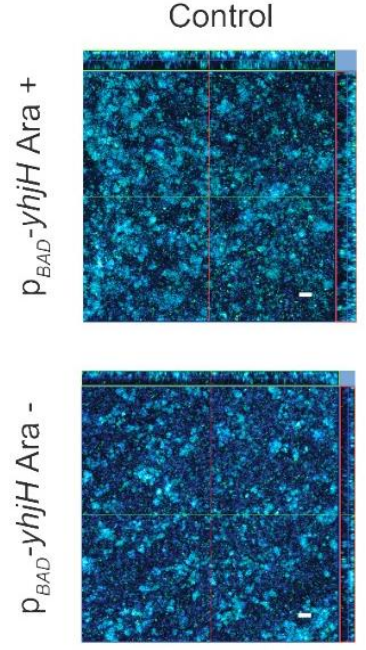

Colistin
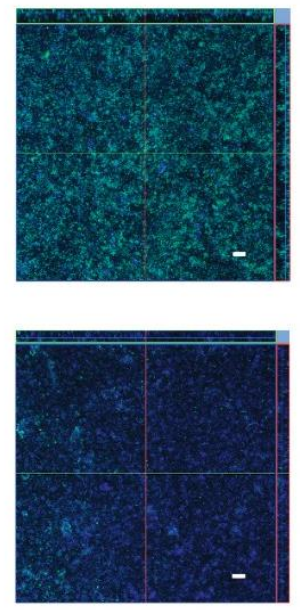

Colistin
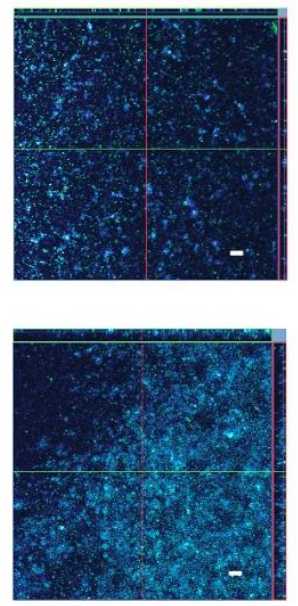

B

PAO1/p ${ }_{B A D}-y h j H$ Resistant vs Parent

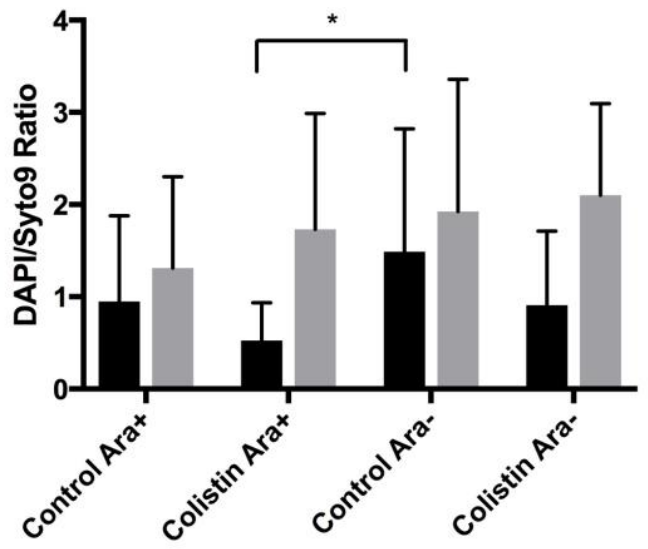

Resistant

Figure 4.6 CLSM images (20x objective) (A) of 3-day-old biofilms of PAO1/p $\mathrm{p}_{B A D}-y h j H$ colistin resistant (Top) and parent (Bottom) strains treated with arabinose 1\% (Ara+) or untreated (Ara-) for 4 hours followed by 24 hours exposure to colistin at $8 \mu \mathrm{g} / \mathrm{ml}$ or not (Control). Biofilms were stained with Syto9 and DAPI prior imaging. Cells appear green; total DNA appears blue and cyan when co-localized. Scale bar is $20 \mu \mathrm{m}$. (B) Bacterial load and total DNA after 24-hour antibiotic exposure reported as DAPI/Syto9. Images from five independent experiments $(\mathrm{N}=5)$ were analyzed to determine dead/live ratios for each condition, presented as average value +/- STDEV. Results analyzed to evaluate eDNA/Cells ratios for each condition in both strains. Statistical significance analysis conducted using two-way ANOVA, corrected by Sidak hypothesis testing ( $p$-value interpretation: $\left.{ }^{*}<0.05\right)$. 
Antimicrobial killing efficacy of colistin and vancomycin against 1-day-old biofilms

The potential for the synergy of the combination of colistin and vancomycin at 0.25 of the MBEC ( 8 and $64 \mu \mathrm{g} / \mathrm{mL}$, respectively) was evaluated against 1-day-old biofilms with or without induced c-di-GMP mediated dispersal (Figure 4.7).

Parent Ara+

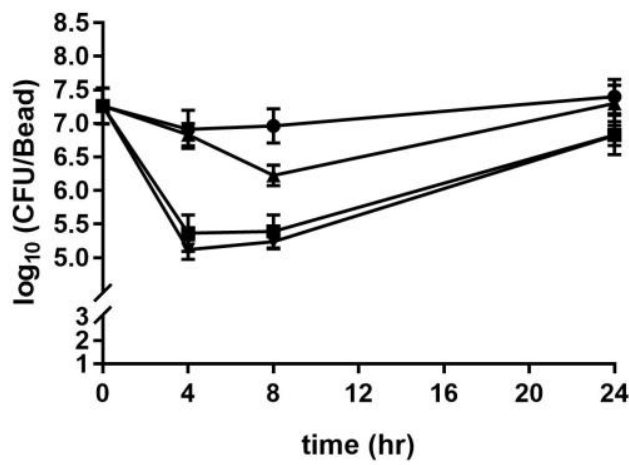

Parent Ara-



Resistant Ara+

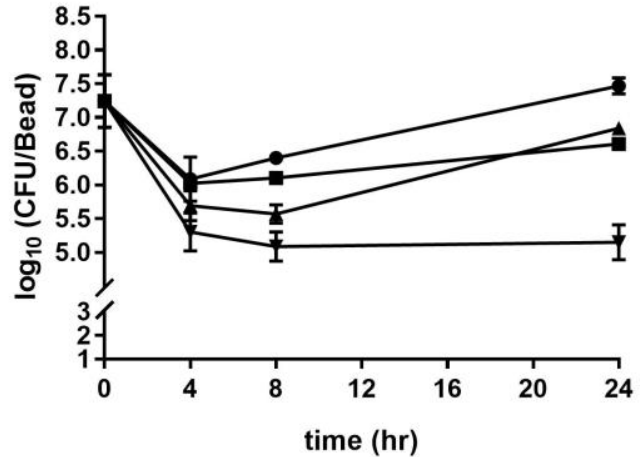

Resistant Ara-

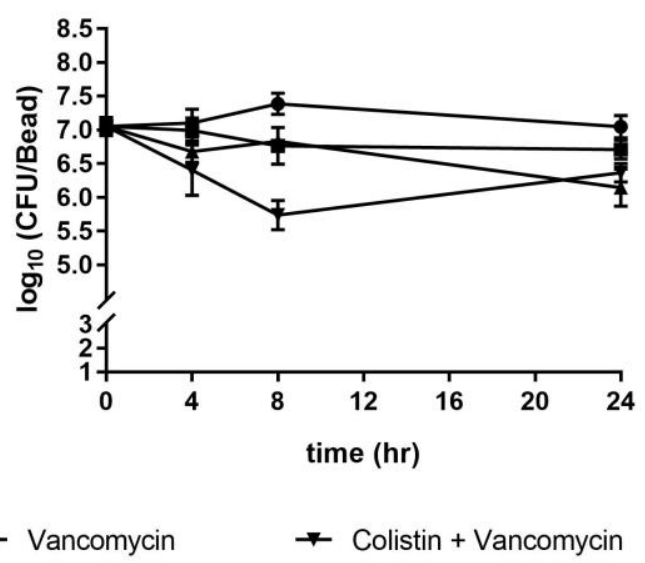

Figure 4.7 C-di-GMP mediated biofilm dispersal induced by arabinose allows synergistic antimicrobial effect for colistin combined with vancomycin against the resistant $\mathrm{PAO} 1 / \mathrm{p}_{B A D}-y h j H$ but not its parent strain. Residual bacterial load $\log _{10}$ (CFU/Bead) after exposing 1-day-grown biofilms of both strains. Biofilms were treated (Ara+) or untreated (Ara-) with $1 \%$ arabinose for 4 hours and then exposed to colistin $(8 \mu \mathrm{g} / \mathrm{mL})$ or vancomycin $(64 \mu \mathrm{g} / \mathrm{mL})$ alone or in combination (at time=0). Experiments conducted in quadruplicates $(\mathrm{N}=4)$. 
Without induced dispersal, the antibiotic combination resulted in a substantial drop in $\log _{10}$ CFU/Bead in the resistant strain following 8 hours of exposure compared to control (5.74 versus 7.38 ). With c-di-GMP mediated dispersal, there was a further drop in values (5.08 versus 6.4), but the drop in the control can mean an additive effect of dispersal itself. In contrast, at the 24-hour time point, resistant strain samples following induced dispersal exhibited a sustained $2 \log _{10}$ drop in CFU/Bead under the combination (5.15 versus 7.46); in the non-dispersed resistant strain, biofilms could recover (6.36 versus 7.5 in the control).

In the parent $\mathrm{p}_{B A D^{-}} y$ hjH strain in the samples with induced dispersal after 4-8 hours, colistin treatment alone results in a drop to 5.36-5.39 $\log _{10}$ CFU/Bead versus controls (6.91-6.96) in the respective time points. Combined antibiotic treatment in these samples resulted in a further decrease at 4-8 hours (5.12-5.24). At the 24-hour time point, both colistin and colistin plus vancomycin treatments contained 6.83-6.82 $\log _{10} \mathrm{CFU/Bead}$ versus 7.4 in the control. The values at the 8-hour time point following dispersal reveal that, as expected, the resistant strain was more susceptible to vancomycin (5.57 $\log _{10}$ CFU/Bead) and the parent strain was more susceptible to colistin (5.39 $\left.\log _{10} \mathrm{CFU} / \mathrm{Bead}\right)$. It is evident then that the $\mathrm{MDK}_{99}$ by this combination at these antibiotic concentrations lies between 8-24 hours, but only following induced dispersal. 


\subsection{Discussion}

Over $80 \%$ of infections are biofilms associated (Burmølle, Thomsen et al. 2010), at the majority of cases biofilms are comprised of multiple pathogenic species, with a various antimicrobial susceptibility pattern. Combination therapy is the most common and efficient strategy to treat these infections (Høiby, Bjarnsholt et al. 2010). However, when infections involve MDR pathogens, such as a colistin resistant $P$. aeruginosa used here, treatment options are limited. One research avenue to combat resistant species is to investigate unconventional strategies, combining drugs which at first sight should not retain any activity against the pathogen (Buyck, Tulkens et al. 2015). These unconventional combinations are typically designed based on predicted mechanisms of resistance. For example, unusual treatment combining daptomycin with trimethoprim was found to be an efficient therapeutic alternative both in-vitro and in-vivo against daptomycin resistant Staphylococcus aureus (S. aureus) (Avery, Steed et al. 2012, Steed, Werth et al. 2012). In the same spirit, a combination of colistin and vancomycin was found to be efficient in-vitro against colistin resistant strains of $P$. aeruginosa, K. pneumonia \& Acinetobacter baumannii (A. baumannii) (Vidaillac, Benichou et al. 2012). The latter has however never been proven efficient against biofilms, which are the most common type of infections.

The objectives of this study were to 1) assess the potential for synergy of the unconventional antimicrobial combinations and 2) evaluate the potential of c-di-GMP mediated biofilm dispersal to increase antimicrobial efficacy; against biofilms of colistin susceptible and resistant strains of $P$. aeruginosa. Dispersal 
of biofilm utilizing this specific cellular mechanism has proven to be a promising strategy to treat biofilms of susceptible strains (Roizman, Vidaillac et al. 2017). The question here is whether this strategy is effective against biofilms of resistant bacteria. More specifically, the potential of c-di-GMP mediated biofilm dispersal was evaluated as a strategy to increase antimicrobial efficacy and eventually reach bactericidal activity against biofilm of MDR $P$. aeruginosa. For the purpose of this study, a pair of susceptible and colistin resistant PAO1/p $\mathrm{p}_{B A D^{-}}$yhjH strains was used as a proof-of-concept.

A colistin resistant derivative of $\mathrm{PAO} 1 / \mathrm{p}_{B A D^{-}} y$ hjH was generated through repeated exposure to increasing sub-inhibitory concentrations of colistin in liquid batch over 14 days, as known from the literature (Cabot, Zamorano et al. 2016). The resulting resistant strain exhibited 5 two-fold decrease in susceptibility to colistin as compared to the parent strain, with a MIC of 32-64 $\mu \mathrm{g} / \mathrm{mL}$ versus initial MIC of $1-2 \mu \mathrm{g} / \mathrm{mL}$ (table 4.2). Several phenotypic differences have been shown between the parent and resistant strains following the evolution of colistin resistance (table 4.1). An increased production of pigment during growth in MHB was noticed, and estimated an increase of up to 4 times in the concentration of pyocyanin in the solution. This phenomenon has been reported in the literature before, following in-vivo exposure to sub-inhibitory concentrations of antibiotics, including colistin (Wright, Fothergill et al. 2013). Additional increase in known virulence factor production, rhamnolipids and proteolytic enzymes, has also been detected; as well as a significant 100 -fold decrease in swimming and 10-fold in swarming motilities, all correlate to colistin resistance development (Finlayson and Brown 2011). It was noticeable that the resistant strain tends to aggregate even 
during growth in liquid batch and grow as a SCV on agar. This is in line with the increase in surface attachment and/or aggregation seen as a result of antibiotic exposure (Hogardt and Heesemann 2010, Thien-Fah Mah 2012, Moradali, Ghods et al. 2017). Colistin stress has thus selected out of the natural heterogenic resistance in the population, both biofilm forming and virulent, SCVs. The higher intracellular c-di-GMP concentration necessary to promote this phenotype might explain the higher effect dispersal via this specific mechanism had on the colistin resistant $\mathrm{PAO} 1 / \mathrm{p}_{B A D^{-}} y$ hiH versus the parent strain.

Whole genome sequencing and pairwise comparison of the evolved resistant strain versus the parent indicated that a significant number of genetic variations had been selected. Genes encoding the proteins PhoQ, TpbA and LpxC demonstrated the most significant alterations to $100 \%$ of sequences. $\mathrm{PhoQ}$ is a sensor kinase protein part of the two-component system PhoPPhoQ (Gellatly, Needham et al. 2012). This system has been linked to cationic antimicrobial resistance before and especially to polymyxin resistance (Gooderham, Gellatly et al. 2009, Gutu, Sgambati et al. 2013). PhoQ/P system controls the enzymatic cascade operon arn that modifies the bacterial Lipid-A, an essential constituent of the bacterial membrane and the target for polymyxins (Miller, Brannon et al. 2011). In-vivo P. aeruginosa isolates from CF with variations in the PhoQ/P system have been described (Gellatly and Hancock 2013, Mustafa, Chalhoub et al. 2016). Variants are reported to have lower motility, higher virulence, and are correlated to increase in inflammatory reactions. An identified nonsense mutation in Amino Acid 435 (Original Glu 435 ), leading to a premature stop, which might have resulted in a 14 amino acid 
shorter truncated version of $\mathrm{PhoQ}$ to be translated. A structural study of PhoQ in $E$. coli has demonstrated that some mutations in this cytoplasmic domain can lead to increased activity (Marina, Mott et al. 2001).

The periplasmic domain of PhoQ interacts with $\mathrm{Mg}^{+2}$ cations via Glutamic acid residues (Prost, Daley et al. 2007). Colistin replaces $\mathrm{Mg}^{+2}$ cations in the interaction with Lipid-A, a necessary step prior to its incorporation in to the membrane causing bacterial cell leakage (Biswas, Brunel et al. 2012). PhoQ is activated by lower $\mathrm{Mg}^{+2}$ concentrations, it phosphorylates PhoP which directly activates translation of the arnBCADTEF-pmrE operon (Fernández, Gooderham et al. 2010, Olaitan, Morand et al. 2014); PhoP also autoregulates its own oprH-phoP-phoQ operon and another two-component system PmrA/B, which also increases expression of the arn operon. Differential expression results presented here indeed showed increased expression of these abovementioned systems. Overexpression of arn is known to provide the highest levels of innate resistance to polymyxins in $P$. aeruginosa (Fernández, Álvarez-Ortega et al. 2013, Lo Sciuto and Imperi 2018); and is achieved by high redundancy of controlling two-component systems.

LpxC is the first enzyme in the cascade that catalyzes the synthesis of Lipid-A, and it is the target for many new tentative bacterial inhibitors (Mdluli, Witte et al. 2006, Tan, Vidaillac et al. 2017). LpxC catalyzes the transformation of UDP-glucuronic acid to 4-amino-arabinose addition (Johnson, Nation et al. 2017). The mutation discovered results in a non-synonymous amino acid change Phe ${ }_{152}$ Leu possibly resulting in decreased production of Lipid-A, as 
described before (Jochumsen, Marvig et al. 2016). Moreover, it would lead to increased virulence by bacterial endotoxins (Gough, Hancock et al. 1996).

The gene that showed the highest number of detected variations was $t p b A$, the product of this gene, a tyrosine phosphatase that normally negatively regulates $T p b B$. TpbB is a $D G C$ that has been linked to increased eDNA production, via increased intracellular c-di-GMP concentration (Ueda and Wood 2010). Mutations in tpbA have been shown to result in an increase of c-di-GMP, due to TpbB activity, and lead to increased production of EPS polysaccharides and SCV phenotype (Ueda and Wood 2009, Pu and Wood 2010). Here, it was shown that no significant changes between parent and resistant strains. A relative change to eDNA production and decrease in the ratio of eDNA/bacteria was observed following 24-hour antibiotic challenge, but remains of low significance. The effect of eDNA on colistin susceptibility is thought to occur through the chelation of $\mathrm{Mg}^{+2}$ of which colistin killing mechanism is dependent (Mulcahy, Charron-Mazenod et al. 2008); it is also known that this effect is negated at $\mathrm{Mg}^{+2}$ rich media such as MHB media.

The original experimental plan for CLSM in this chapter was to estimate eDNA and PsI ratios versus live cells in the parent and derivative strains. DAPI was used for staining total DNA (blue channel), SYTO9 for live cells (green channel) and the PsI binding fluorescent stain Texas-Red ${ }^{\circledR}$-conjugated Concanavalin (Molecular Probes, USA) was used in the red channel. Unfortunately, the protocol to use the latter Psl stain could not be optimized for this experiment based on 8-well slides, but did not hinder the staining and analysis of the former two channels. Recently this stain was successfully 
applied to $P$. aeruginosa using an in-vitro "slide biofilm" model (Chin, Sinha et al. 2017), and is still believed to be useful to attempt to repeat it for this set of experiments. Considering that eDNA is one of the most important negatively charged constituents of the EPS, a decrease in its production relative to Psl would cause the EPS to become more of a cationic nature, possibly inhibiting colistin diffusion based on an electric charge (Batoni, Maisetta et al. 2016). The hypothesis is that higher intracellular c-di-GMP will lead to more biofilm and EPS formation (Harmsen, Yang et al. 2010), with lower eDNA content (Ueda and Wood 2010), better protect from polymyxin stress. Apart from exemplifying the ability of $P$. aeruginosa to adapt to changing environmental conditions and stresses, it is necessary to perform more experiments to describe the connections between c-di-GMP mediated dispersal and antibiotic susceptibility better.

The genetic profile and differential gene expression evident here have been described in detail in the literature (Moskowitz, Ernst et al. 2004, Fernández, Gooderham et al. 2010, Moskowitz, Brannon et al. 2012). Colistin resistance, has been shown to be multi-component (Jochumsen, Marvig et al. 2016): mutations in genes of $P h o P Q, p m r A B \& \operatorname{lp} x C$ systems lead to upregulation of the arnBCADTEF-pmrE operon (Gunn, Ryan et al. 2000, Barrow and Kwon 2009). Additionally, it has been shown that mutations in only mechanisms pmrAB or phoPQ by themselves were not sufficient for the highest resistance phenotype evident (Jochumsen, Marvig et al. 2016). Rather than a redundant effect, differential expression and upregulation of both systems was shown to be complementary. Recently, a morbidostat, continuous culture system was used to examine the kinetics of accumulation of mutations and differential 
gene expression in $P$. aeruginosa clinical isolates from colistin treated CF patients (Regenbogen, Willmann et al. 2017). The results concur with gene expression results shown in this work, mutations in $P h o P Q$ and $p m r A B$ systems lead to the higher expression of the arn operon. $P$. aeruginosa exhibits a heterogenic resistance to colistin initially, the evolution of resistance to higher concentrations of colistin occurs following 7-12 days of drug exposure, in biofilms and planktonic cultures; consistent with evidence from chapter 3 , and in this chapter.

Considerable changes to the MIC profile are evident when comparing the colistin resistant PAO1/p $\mathrm{p}_{B A D^{-}} y$ hjH and its parent. Development of resistance to one polymyxin (colistin) has resulted in an increase of resistance to another polymyxin antibiotic, showing, at least in part, that resistance mechanisms apply to every group of antibiotics, once resistance to one is established (Olaitan, Morand et al. 2014). The consistent increase in susceptibility to virtually all $\beta$-lactam antibiotics tested has proved the existence of the "Antibiotic Seesaw Effect", in this strain as a direct result of colistin exposure. In a similar manner to what has been shown in Gram-positive organisms, the emerging resistance to drugs which target the bacterial membrane and cell wall, such as daptomycin and $\beta$-lactams (Vignaroli, Rinaldi et al. 2011). Structural changes to these cellular barriers enable a considerable increase in antimicrobial efficacy for one group when resistance has developed for the other, and vice versa (Ortwine, Werth et al. 2013). The structural similarity and mechanisms of action between daptomycin and colistin necessitate the "Antibiotic Seesaw Effect" to be taken into account when developing potential solutions for colistin resistance. Furthermore, it was shown here that a 
considerable drop in resistance of $P$. aeruginosa to vancomycin, an antimicrobial drug used to treat Gram-positive infections becomes feasible using the "Seesaw Effect".

The effect of the biofilm mode of growth in regards to susceptibility has been substantial; the differences in MIC were almost wholly diminished, except for a handful of antibiotics (Table 4.4). According to results, carbapenems, tobramycin, piperacillin and rifampicin are shown to be the most promising treatments within the antibiotics tested to tackle colistin resistance. The potential for synergy of vancomycin and colistin against the parent and resistant strains was shown as a promising strategy for planktonic cultures (Vidaillac, Benichou et al. 2012). Here, the "seesaw effect" was not observed in biofilms initially; it was shown here via biofilm time/kill analysis, that synergy between colistin and vancomycin is only possible following induced c-di-GMP mediated dispersal (Figure 4.7). This demonstrated the potential of biofilm dispersal as a therapeutic strategy to prevent and eliminate the development of colistin resistance in $P$. aeruginosa, the suggested mechanism to this synergy is presented as a scheme in figure 4.8 below.

The main limitation of this research is the small number of strains, a parent, and a resistant $\mathrm{PAO} 1 / \mathrm{p}_{B A D^{-}} y$ hiH strain. There is a possibility that the effects that shown here are limited to $P$. aeruginosa, and it cannot be ruled out that the concept of c-di-GMP mediated dispersal would involve other factors in other species. However, it was already reported that a stronger synergistic effect of the vancomycin and colistin combination against $K$. pneumonia and A. baumannii, two major human pathogens (Vidaillac, Benichou et al. 2012). 
Further research is now warranted to confirm the potential of c-di-GMP mediated biofilm dispersal and vancomycin combination with colistin against biofilms of both pathogen species.

In conclusion, colistin resistance in $P$. aeruginosa seems to result in increased susceptibility to vancomycin, which is reminiscent of the "Seesaw Effect" described in Gram-positive bacteria. Data analysis suggests that synergy may result from heterogeneous colistin resistance, in both colistin resistant and parent strains. Nonetheless, the colistin resistant population demonstrates increased susceptibility to vancomycin (among other antibiotics) and is enriched as compared to the colistin susceptible strain. In the presence of both drugs, colistin will target the colistin susceptible population, which is resistant to vancomycin, and on the other hand, vancomycin will kill the colistin resistant population that demonstrates an increased susceptibility to vancomycin. Dispersal is instrumental in utilizing the seesaw effect in biofilm bacteria, as it facilitates access to both drugs to the different populations. The seesaw effect observed in both Gram-positive and Gram-negative bacteria should be further investigated as it has a high potential for treatment. An attempt to develop a dispersal treatment via c-di-GMP depletion, based on this proof-of-concept study is both plausible and essential. 


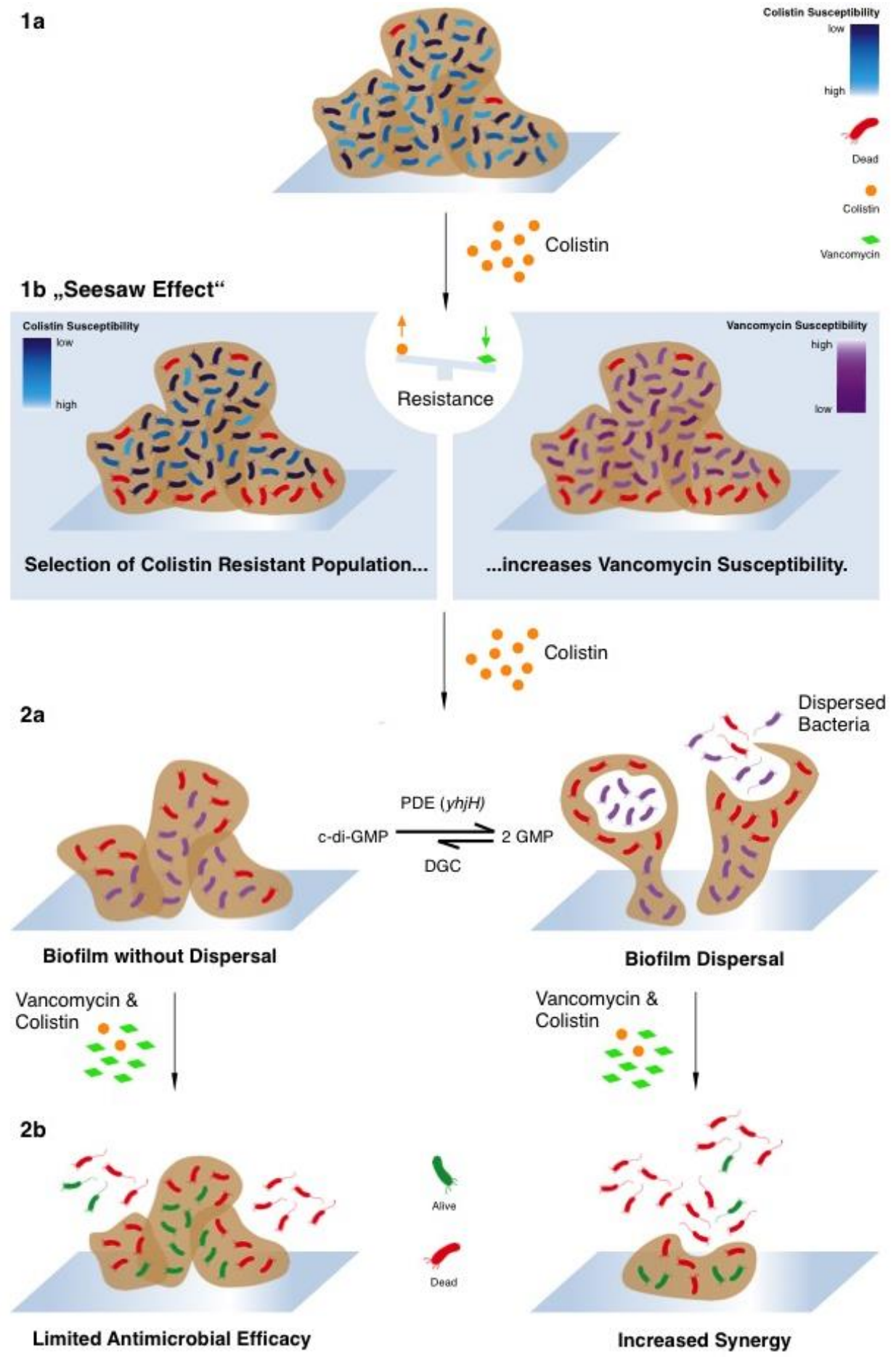

Figure 4.8 Schematic description of the combined effect of c-di-GMP mediated biofilm dispersal and the "seesaw effect". 1a. Colistin exposure and sub-optimal concentrations result in enrichment of the colistin resistant sub-population. $\mathbf{1 b}$. Resistant subpopulations become more susceptible to vancomycin. 2a. Biofilm dispersal is crucial to enable penetration of both drugs through the EPS. $\mathbf{2 b}$. Combined vancomycin and colistin treatment following dispersal has the potential for bactericidal effect. 


\section{Conclusions and Future Prospects}

Bacterial biofilms are considered difficult to treat due to (1) slow or impaired penetration of antimicrobials in their EPS matrix and (2) the presence of different sub-populations existing at different metabolic states and exhibiting different susceptibility profiles within the same community. Antimicrobial combinations have been proven to be the recommended strategy to treat biofilms as they exhibit superior efficacy as compared to mono-treatments (Černohorská and Votava 2008, Herrmann, Yang et al. 2010, Høiby, Bjarnsholt et al. 2010, Hengzhuang, Wu et al. 2011). Despite the increased use of combined treatments, antimicrobial killing efficiency often remains limited. Novel research is focused at the development of adjunct therapy capable of increasing the efficacy of synergetic compounds. Over the past decades, numerous studies have suggested the enormous potential of dispersal, by various means, to eradicate biofilms (Banin, Brady et al. 2006, Landini, Antoniani et al. 2010, Barraud, J. Kelso et al. 2015).

In this thesis, c-di-GMP mediated biofilm dispersal was evaluated as a therapeutic strategy to enhance antimicrobial efficacy against $P$. aeruginosa biofilms. Proof-of-concept studies were designed and successfully achieved to demonstrate that (1) c-di-GMP mediated biofilm dispersal enhances antimicrobial synergy, (2) does not increase the risk for resistant variant selection and (3) is crucial for achieving an efficient killing effect which are attainable, even when MDR strains are involved. 
First, using a $P$. aeruginosa laboratory construct strain, $P A O 1 / \mathrm{p}_{B A D^{-}} y h j H$, carrying the PDE gene inducible upon addition of arabinose. The induced drop in intracellular c-di-GMP resulted in a maximum drop of $1 \log _{10} \mathrm{CFU} / \mathrm{Bead}$, significant, but the majority of the biofilm population remains and regrows even under consecutive expression. It was demonstrated that the synergistic combination of imipenem and tobramycin is enhanced following YhjH induction in mature biofilms. The induction of $Y h j H$ must hamper antimicrobial biofilm tolerance mechanisms, which are c-di-GMP regulated (Ha and O'Toole 2015); significant and sustained killing effect was achieved in-vitro at physiological relevant antimicrobial concentrations only following induction.

To further evaluate the potential of c-di-GMP mediated dispersal as a possible therapeutic strategy, the risk for development of variants or sub-populations was assessed with tolerant or resistant phenotypes. Biofilm variants emerging during or following in-vitro or in-vivo colistin therapy is a well-described phenomenon (Wright, Fothergill et al. 2013, El-Halfawy and Valvano 2015, Brauner, Fridman et al. 2016, Lee, Park et al. 2016, Regenbogen, Willmann et al. 2017). It has been shown in-vivo, that in many clinical conditions, such as in CF patients lung sputum, the phenomenon is caused by the inability to reach adequate colistin concentrations (Beringer 2001, Moskowitz, Brannon et al. 2012, Lee, $\mathrm{Na}$ et al. 2014, Mustafa, Chalhoub et al. 2016, Grégoire, Aranzana-Climent et al. 2017). Using colistin as a model treatment, this work demonstrated that the emergence and morphotypes of variants are similar in dispersed and non-dispersed biofilms. However, preliminary results suggest that the minimal duration for experimental colistin resistance evolution in-vitro should be extended. The minimum reported time until resistance development 
in planktonic cultures is 12-14 days (Lee, Park et al. 2016). The "Bead biofilm" protocol was utilized here for time/kill experiments and variant selection. It allowed for reproducible samples of biofilms as shown here for $P$. aeruginosa at $10^{7} \mathrm{CFU} /$ bead, in high-throughput manner. A future experiment, employing this system for AMR phenotypes, variant selection, also by repeated subinhibitory exposure technique, should be considered. Differential gene expression of the biofilm forming fraction in samples and possible biofilmspecific mutations are foreseeable.

The potential of c-di-GMP mediated biofilm dispersal was further evaluated against biofilms of MDR strains. A colistin resistant derivative of our construct PAO1/ $\mathrm{p}_{B A D^{-}} y$ hjH was generated. Of interest and consistent with the literature, the colistin resistant strain exhibits an "unconventional" drug susceptibility profile, showing increased susceptibility to drugs such as vancomycin or carbapenems. This effect was reminiscent to the previously described "Antibiotic Seesaw Effect", whereby increased resistance to one class of antibiotics results in increased susceptibility to other classes (Vignaroli, Rinaldi et al. 2011, Vidaillac, Benichou et al. 2012, Ortwine, Werth et al. 2013, Barber, Ireland et al. 2014). Taking advantage of this phenotype, unconventional drug combinations have been tested and successfully developed to treat infections caused by MDR bacteria. The most studied example is infections caused by vancomycin-intermediate resistant MRSA successfully treated by vancomycin and $\beta$-lactam combinations (Sieradzki and Tomasz 1997, Vignaroli, Rinaldi et al. 2011, Ortwine, Werth et al. 2013, Barber, Ireland et al. 2014, Dilworth, Ibrahim et al. 2014). A similar phenomenon was suggested in the literature with colistin resistance in Gram-negative bacteria, and strangely enough, 
colistin and vancomycin combination was found to be efficient and synergistic against cultures of $P$. aeruginosa and K. pneumonia (Vidaillac, Benichou et al. 2012). Unfortunately, there was no data available regarding increased efficacy in the less favorable scenario of biofilms. In this work, it was demonstrated that the combination of vancomycin and colistin is synergistic only against the colistin resistant derivative strain. However, synergy can be achieved only following induced biofilm dispersal supporting the therapeutic potential of this strategy. More conventional combinations against colistin resistant strains should be considered as the difference in susceptibility profile suggests; a combinatorial treatment of colistin and carbapenems for example.

To further gain insights on the mechanism by which the colistin resistant derivative strain becomes more susceptible to unconventional therapy in planktonic cultures and biofilm settings, whole-genome sequencing was performed for the parent $\mathrm{PAO} 1 / \mathrm{p}_{B A D^{-}} y$ hjH versus its colistin resistant derivative. Dominant point mutations in genes encoding proteins regulating or involved in the biosynthesis of Lipid-A were identified. Amongst them, mutations in $\operatorname{tp} b A$, a gene related to the production of eDNA and other matrix related components was found. The two-component system TpbA/B has been found to regulate an antibiotic resistant $P$. aeruginosa SCV phenotype (Valentini and Filloux 2016), as was evident in this work. The tpbA mutant phenotype has been shown before to culminate in a high intercellular c-di-GMP content in cells, due to TpbB DGC activity (Pu and Wood 2010). If the colistin resistant evolved strain SCV phenotype is dependent upon high intercellular c-di-GMP equilibrium, induction of the PDE YhjH would have more impact on those tolerance mechanisms. 
Other mutations with frequencies of $70-90 \%$ were found to be in the locus PA0727 (Supplementary Table 1), which is related to the Pf filamentous phage in PAO1 (Martínez and Campos-Gómez 2016). This gene is known to be hypermutable and may play a key role in changing the PAO1 EPS constituents, and also promote SCV phenotypes (Kirisits, Prost et al. 2005). These mutations might contribute to the observed differences between colistin susceptible and resistant derivative strains and should be further investigated.

Point mutations in $p h o Q$ and $l p x C$ were identified in the colistin resistant derivative strain, as is commonly observed in literature (Lee, Park et al. 2016). These two genes are involved in the biosynthesis of lipid-A located in the bacterial membranes and the biological target of colistin. These led to a different differential gene expression in the resistant strain: upregulation of the whole arnBCADTEF-pmrE operon (Fernández, Gooderham et al. 2010) was evident. Through catalyzed action of this enzymatic cascade transcribed by the operon, the modifications hide the negative charge of Lipid-A and limit binding of polymyxins (Breidenstein, de la Fuente-Núñez et al. 2011, Miller, Brannon et al. 2011). The PhoP/Q and PmrA/B two-component systems, are central signal transduction systems, variations in their expression also were shown to result in changes to other cellular attributes, such as known virulence factor production and motility, leading to a SCV phenotype (Rodrigue, Quentin et al. 2000). It has been suggested that excretion of bacterial Lipid-A increases virulence, as it is a main component of LPS (Maldonado, Sá-Correia et al. 2016). 
The product of the plasmid mediated colistin resistance mcr-1 gene, is a phosphoethanolamine transferase, which was shown in E. coli to catalyze the addition of phosphoethanolamine to Lipid-A (Liu, Wang et al. 2016). This plasmid has been since shown to be transmittable to all Gram-negative ESKAPE (Enterococcus faecium, S. aureus, K. pneumoniae, A. baumannii, P. aeruginosa, and Enterobacter species) pathogens which are often treated with colistin (Liu, Chandler et al. 2017). The mcr-1 enzyme has been shown to increase resistance in $K$. pneumoniae \& $A$. baumannii but not in $P$. aeruginosa. K. pneumoniae and $P$. aeruginosa both have the arn pathway for arabinose addition in Lipid-A and $A$. baumannii has its own phosphoetnanolamine transferase, PmrC (Adams, Nickel et al. 2009). It is safe to assume that resistance to colistin, inherited or via horizontal gene transfer does constitute a change to Lipid-A and thus changes the properties of the outer membrane. This change will thus facilitate a significant change to the susceptibility profile of resistant strains as a "seesaw effect".

Once the new potential for antibiotic combinations increases, induced dispersal via depletion of intracellular c-di-GMP, was shown here to allow sufficient penetration and synergy. Future experiments with clinical strains and other bacterial species, such as $K$. pneumonia and A. baumannii, are now warranted; to assess the potential of c-di-GMP mediated biofilm dispersal further, to prove that the observed eradication by antimicrobial combinations is not limited to this strain alone. Work was initiated on transforming K. pneumonia KP1 with the plasmids pJN105 and $\mathrm{p}_{B A D^{-}} y$ hjH. Evaluation of unconventional treatments and c-di-GMP mediated biofilm dispersal as a 
strategy to treat multi-species biofilms of ESKAPE such as $P$. aeruginosa and S. aureus.

Experiments described in this work were based on static biofilm models. Even though these models enable high-throughput investigation of drug combinations, there is a need to examine the effects of organisms growing in continuous culture conditions. Ongoing experiments utilizing the DTU flow cell system (data not shown) with the PAO1/p $\mathrm{p}_{B A D^{-}} y$ hjH treated with different antimicrobial combinations following dispersal exhibit a positive trend for biofilm eradication, although further experimentation and optimization are warranted. Additionally, the results shown in this thesis could serve as the basis for future experiments using the CDC biofilm reactor (Larimer, Winder et al. 2016) to study the effect on biofilms when exposed to antimicrobial concentrations mimicking therapeutic regimes.

Finally, following these proof-of-concept studies, c-di-GMP mediated biofilm dispersal should now be tested with potential biofilm dispersal agents. Experimentation with different enzymes that are known to facilitate dispersal, such as DNAse and PsIG (Yu, Su et al. 2015, Gonzalez Moreno, Trampuz et al. 2017) is ongoing; results are promising, showing similar trends as with the $\mathrm{p}_{B A D^{-}} y h j \mathrm{H}$ construct. 


\section{References}

Adams, M. D., G. C. Nickel, S. Bajaksouzian, H. Lavender, A. R. Murthy, M. R. Jacobs and R. A. Bonomo (2009). "Resistance to Colistin in Acinetobacter baumannii Associated with Mutations in the PmrAB Two-Component System." Antimicrobial Agents and Chemotherapy 53(9): 3628-3634.

Allegranzi, B., S. B. Nejad, C. Combescure, W. Graafmans, H. Attar, L. Donaldson and D. Pittet (2010). "Burden of endemic health-care-associated infection in developing countries: systematic review and meta-analysis." The Lancet 377(9761): 228-241.

Alvarez-Ortega, C. and C. S. Harwood (2007). "Responses of Pseudomonas aeruginosa to low oxygen indicate that growth in the cystic fibrosis lung is by aerobic respiration." Molecular microbiology 65(1): 153-165.

Amin, N. E., C. G. Giske, S. Jalal, B. Keijser, G. Kronvall and B. Wretlind (2005). "Carbapenem resistance mechanisms in Pseudomonas aeruginosa: alterations of porin OprD and efflux proteins do not fully explain resistance patterns observed in clinical isolates." APMIS 113(3): 187-196.

Amyes, S. G. B., R. S. Miles, C. J. Thomson and G. Tillotson (1996). Antimicrobial Chemotherapy: Pocketbook. United Kingdom, Taylor \& Francis Ltd.

Anders, S. and W. Huber (2010). "Differential expression analysis for sequence count data." Genome Biology 11(10): R106.

Avery, L. M., M. E. Steed, A. E. Woodruff, M. Hasan and M. J. Rybak (2012). "Daptomycin-Nonsusceptible Vancomycin-Intermediate Staphylococcus aureus Vertebral Osteomyelitis Cases Complicated by Bacteremia Treated with High-Dose Daptomycin and Trimethoprim-Sulfamethoxazole." Antimicrobial Agents and Chemotherapy 56(11): 5990-5993.

Bakthavatchalam, Y. D., A. K. Pragasam, I. Biswas and B. Veeraraghavan (2018). "Polymyxin susceptibility testing, interpretative breakpoints and resistance mechanisms: An update." Journal of Global Antimicrobial Resistance 12: 124-136.

Banin, E., K. M. Brady and E. P. Greenberg (2006). "Chelator-Induced Dispersal and Killing of Pseudomonas aeruginosa Cells in a Biofilm." Applied and Environmental Microbiology 72(3): 2064-2069.

Barber, K. E., C. E. Ireland, N. Bukavyn and M. J. Rybak (2014). "Observation of "Seesaw Effect" with Vancomycin, Teicoplanin, Daptomycin and Ceftaroline in 150 Unique MRSA Strains." Infectious Diseases and Therapy 3(1): 35-43. 
Barraud, N., M. J. Kelso, S. A. Rice and S. Kjelleberg (2015). "Nitric Oxide: A Key Mediator of Biofilm Dispersal with Applications in Infectious Diseases." Current Pharmaceutical Design 21(1): 31-42.

Barraud, N., D. Schleheck, J. Klebensberger, J. S. Webb, D. J. Hassett, S. A. Rice and S. Kjelleberg (2009). "Nitric Oxide Signaling in Pseudomonas aeruginosa Biofilms Mediates Phosphodiesterase Activity, Decreased Cyclic Di-GMP Levels, and Enhanced Dispersal." Journal of Bacteriology 191(23): 7333-7342.

Barrow, K. and D. H. Kwon (2009). "Alterations in Two-Component Regulatory Systems of phoPQ and pmrAB Are Associated with Polymyxin B Resistance in Clinical Isolates of Pseudomonas aeruginosa." Antimicrobial Agents and Chemotherapy 53(12): 5150-5154.

Batoni, G., G. Maisetta and S. Esin (2016). "Antimicrobial peptides and their interaction with biofilms of medically relevant bacteria." Biochim Biophys Acta 1858(5): 1044-1060.

Beloin, C., S. Renard, J.-M. Ghigo and D. Lebeaux (2014). "Novel approaches to combat bacterial biofilms." Current Opinion in Pharmacology 18: 61-68.

Berditsch, M., T. Jäger, N. Strempel, T. Schwartz, J. Overhage and A. S. Ulrich (2015). "Synergistic Effect of Membrane-Active Peptides Polymyxin B and Gramicidin S on Multidrug-Resistant Strains and Biofilms of Pseudomonas aeruginosa." Antimicrobial Agents and Chemotherapy 59(9): 5288-5296.

Beringer, P. (2001). "The clinical use of colistin in patients with cystic fibrosis." Current Opinion in Pulmonary Medicine 7(6): 434-440.

Billings, N., M. Ramirez Millan, M. Caldara, R. Rusconi, Y. Tarasova, R. Stocker and K. Ribbeck (2013). "The Extracellular Matrix Component Psl Provides Fast-Acting Antibiotic Defense in Pseudomonas aeruginosa Biofilms." PLOS Pathogens 9(8): e1003526.

Biswas, S., J. M. Brunel, J. C. Dubus, M. Reynaud-Gaubert and J. M. Rolain (2012). "Colistin: an update on the antibiotic of the 21st century." Expert Review of Antiinfective Therapy 10(8): 917-934.

Bjarnsholt, T., M. Alhede, M. Alhede, S. R. Eickhardt-Sørensen, C. Moser, M. Kühl, P. $\varnothing$. Jensen and N. Høiby (2013). "The in vivo biofilm." Trends in Microbiology 21(9): 466-474.

Bjarnsholt, T., T. Tolker-Nielsen, N. Høiby and M. Givskov (2010). "Interference of Pseudomonas aeruginosa signalling and biofilm formation for infection control." Expert Reviews in Molecular Medicine 12: e11.

Bloomfield, C., C. E. Staatz, S. Unwin and S. Hennig (2016). "Assessing Predictive Performance of Published Population Pharmacokinetic Models of Intravenous Tobramycin in Pediatric Patients." Antimicrobial Agents and Chemotherapy 60(6): 3407-3414. 
Brauner, A., O. Fridman, O. Gefen and N. Q. Balaban (2016). "Distinguishing between resistance, tolerance and persistence to antibiotic treatment." Nature Reviews Microbiology 14(5): 320-330.

Breidenstein, E. B. M., C. de la Fuente-Núñez and R. E. W. Hancock (2011). "Pseudomonas aeruginosa: all roads lead to resistance." Trends in Microbiology 19(8): 419-426.

Bueno, J. (2014). "Anti-Biofilm Drug Susceptibility Testing Methods: Looking for New Strategies against Resistance Mechanism." J Microbial Biochem Technol 0(0): 10.

Bulitta, J. B., N. S. Ly, C. B. Landersdorfer, N. A. Wanigaratne, T. Velkov, R. Yadav, A. Oliver, L. Martin, B. S. Shin, A. Forrest and B. T. Tsuji (2015). "Two Mechanisms of Killing of Pseudomonas aeruginosa by Tobramycin Assessed at Multiple Inocula via Mechanism-Based Modeling." Antimicrobial Agents and Chemotherapy 59(4): 23152327.

Burkhardt, O., C. Lehmann, R. Madabushi, V. Kumar, H. Derendorf and T. Welte (2006). "Once-daily tobramycin in cystic fibrosis: better for clinical outcome than thrice-daily tobramycin but more resistance development?" Journal of Antimicrobial Chemotherapy 58(4): 822-829.

Burmølle, M., T. R. Thomsen, M. Fazli, I. Dige, L. Christensen, P. Homøe, M. Tvede, B. Nyvad, T. Tolker-Nielsen, M. Givskov, C. Moser, K. Kirketerp-Møller, H. K. Johansen, N. Høiby, P. Ø. Jensen, S. J. Sørensen and T. Bjarnsholt (2010). "Biofilms in chronic infections - a matter of opportunity - monospecies biofilms in multispecies infections." FEMS Immunology \& Medical Microbiology 59(3): 324-336.

Buyck, J. M., P. M. Tulkens and F. Van Bambeke (2015). "Activities of Antibiotic Combinations against Resistant Strains of Pseudomonas aeruginosa in a Model of Infected THP-1 Monocytes." Antimicrobial Agents and Chemotherapy 59(1): 258-268.

Cabot, G., L. Zamorano, B. Moyà, C. Juan, A. Navas, J. Blázquez and A. Oliver (2016). "Evolution of Pseudomonas aeruginosa Antimicrobial Resistance and Fitness under Low and High Mutation Rates." Antimicrobial Agents and Chemotherapy 60(3): 1767-1778.

Candeias, L. P., D. P. S. MacFarlane, S. McWhinnie, L. W., N. L. Maidwell, C. A. Roeschlaub, P. G. Sammes and R. Whittlesey (1998). "The catalysed NADH reduction of resazurin to resorufin." Journal of the Chemical Society, Perkin Transactions 2(11): 2333-2334.

CDC. (2017, December 4, 2017). "Tracking mor Genes, an Emerging Threat." Emerging Drug Resistance Retrieved November 2017, 2017, from www.cdc.gov/drugresistance/tracking-mcr1.html. 
Ceri, H., M. E. Olson, C. Stremick, R. R. Read, D. Morck and A. Buret (1999). "The Calgary Biofilm Device: New Technology for Rapid Determination of Antibiotic Susceptibilities of Bacterial Biofilms." Journal of Clinical Microbiology 37(6): 17711776.

Černohorská, L. and M. Votava (2008). "Antibiotic synergy against biofilm-forming Pseudomonas aeruginosa." Folia Microbiologica 53(1): 57-60.

Chang, C. Y., T. Krishnan, H. Wang, Y. Chen, W. F. Yin, Y. M. Chong, L. Y. Tan, T. M. Chong and K. G. Chan (2014). "Non-antibiotic quorum sensing inhibitors acting against $\mathrm{N}$-acyl homoserine lactone synthase as druggable target." Scientific Reports 4: 7245 .

Chatterjee, M., C. P. Anju, L. Biswas, V. Anil Kumar, C. Gopi Mohan and R. Biswas (2016). "Antibiotic resistance in Pseudomonas aeruginosa and alternative therapeutic options." International Journal of Medical Microbiology 306(1): 48-58.

Chen, W., Y.-M. Zhang and C. Davies (2016). "Penicillin-Binding Protein 3 Is Essential for Growth of Pseudomonas aeruginosa." Antimicrobial Agents and Chemotherapy 61(1): e01651-01616.

Chin, J., S. Sinha, A. Nalaparaju, J. Yam, Z. Qin, L. Ma, Z.-X. Liang, L. Lu, S. Bhattacharjya and L. Yang (2017). "Pseudomonas aeruginosa Psl Exopolysaccharide Interacts with the Antimicrobial Peptide LG21." Water 9(9): 681.

Chow, S., K. Gu, L. Jiang and A. Nassour (2011). "Salicylic acid affects swimming, twitching and swarming motility in Pseudomonas aeruginosa, resulting in decreased biofilm formation." Journal of experimental microbiology and immunology 15: 22-29.

Chua, S. L., L. D. Hultqvist, M. Yuan, M. Rybtke, T. E. Nielsen, M. Givskov, T. TolkerNielsen and L. Yang (2015). "In vitro and in vivo generation and characterization of Pseudomonas aeruginosa biofilm-dispersed cells via c-di-GMP manipulation." Nature Protocols 10(8): 1165-1180.

Chua, S. L., Y. Liu, J. K. H. Yam, Y. Chen, R. M. Vejborg, B. G. C. Tan, S. Kjelleberg, T. Tolker-Nielsen, M. Givskov and L. Yang (2014). "Dispersed cells represent a distinct stage in the transition from bacterial biofilm to planktonic lifestyles." Nature Communications 5: 4462.

Chua, S. L., S. Y.-Y. Tan, M. T. Rybtke, Y. Chen, S. A. Rice, S. Kjelleberg, T. TolkerNielsen, L. Yang and M. Givskov (2013). "Bis-(3' -5' )-Cyclic Dimeric GMP Regulates Antimicrobial Peptide Resistance in Pseudomonas aeruginosa." Antimicrobial Agents and Chemotherapy 57(5): 2066-2075.

Chua, S. L., J. K. H. Yam, P. Hao, S. S. Adav, M. M. Salido, Y. Liu, M. Givskov, S. K. Sze, T. Tolker-Nielsen and L. Yang (2016). "Selective labelling and eradication of antibiotic-tolerant bacterial populations in Pseudomonas aeruginosa biofilms." Nature Communications 7: 10750. 
Chua, S. L., L. Yang, Y. Li, H. J. Ting, G. S. Kohli, Z. Cai, P. Suwanchaikasem, K. G. Kau, Kelvin, S. P. Ng, T. Tolker-Nielsen, L. Yang and M. Givskov (2017). "Reduced Intracellular c-di-GMP Content Increases Expression of Quorum Sensing-Regulated Genes in Pseudomonas aeruginosa." Frontiers in Cellular and Infection Microbiology 7(451).

Ciofu, O., T. Jensen, T. Pressler, H. K. Johansen, C. Koch and N. Høiby (1996). "Meropenem in cystic fibrosis patients infected with resistant Pseudomonas aeruginosa or Burkholderia cepacia and with hypersensitivity to $\beta$-lactam antibiotics." Clinical Microbiology and Infection 2(2): 91-98.

CLSI (2012). M07-A9. Methods for Dilution Antimicrobial Susceptibility Tests for Bacteria That Grow Aerobically. Wayne, PA, Clinical and Laboratory Standards Institute.

Colvin, K. M., V. D. Gordon, K. Murakami, B. R. Borlee, D. J. Wozniak, G. C. Wong and M. R. Parsek (2011). "The pel polysaccharide can serve a structural and protective role in the biofilm matrix of Pseudomonas aeruginosa." PLoS pathogens 7(1): e1001264.

Colvin, K. M., Y. Irie, C. S. Tart, R. Urbano, J. C. Whitney, C. Ryder, P. L. Howell, D. J. Wozniak and M. R. Parsek (2012). "The Pel and Psl polysaccharides provide Pseudomonas aeruginosa structural redundancy within the biofilm matrix." Environmental microbiology 14(8): 1913-1928.

Conly, J. M. and B. L. Johnston (2006). "Colistin: The Phoenix Arises." The Canadian Journal of Infectious Diseases \& Medical Microbiology 17(5): 267-269.

Costerton, J. W., G. G. Geesey and K. J. Cheng (1978). "How bacteria stick." Sci Am 238(1): 86-95.

Cui, P., H. Niu, W. Shi, S. Zhang, H. Zhang, J. Margolick, W. Zhang and Y. Zhang (2016). "Disruption of Membrane by Colistin Kills Uropathogenic Escherichia coli Persisters and Enhances Killing of Other Antibiotics." Antimicrobial Agents and Chemotherapy 60(11): 6867-6871.

D'Argenio, D. A., M. W. Calfee, P. B. Rainey and E. C. Pesci (2002). "Autolysis and Autoaggregation in Pseudomonas aeruginosa Colony Morphology Mutants." Journal of Bacteriology 184(23): 6481-6489.

Dalecki, A. G., C. L. Crawford and F. Wolschendorf (2016). "Targeting Biofilm Associated Staphylococcus aureus Using Resazurin Based Drug-susceptibility Assay." Journal of Visualized Experiments : JoVE(111): 53925.

Davies, E. V., C. E. James, M. A. Brockhurst and C. Winstanley (2017). "Evolutionary diversification of Pseudomonas aeruginosa in an artificial sputum model." BMC microbiology 17(3): e12866-12816. 
Denton, M., K. Kerr, L. Mooney, V. Keer, A. Rajgopal, K. Brownlee, P. Arundel and S. Conway (2002). "Transmission of colistin-resistant Pseudomonas aeruginosa between patients attending a pediatric cystic fibrosis center." Pediatric Pulmonology 34(4): 257-261.

Deretic, V. (2000). Pseudomonas aeruginosa infections. Persistent Bacterial Infections. J. P. Nataro, M. J. Blaser and S. Cunningham-Rundles, American Society of Microbiology: 305-326.

Dilworth, T. J., O. Ibrahim, P. Hall, J. Sliwinski, C. Walraven and R.-C. Mercier (2014). " $\beta$-Lactams Enhance Vancomycin Activity against Methicillin-Resistant Staphylococcus aureus Bacteremia Compared to Vancomycin Alone." Antimicrobial Agents and Chemotherapy 58(1): 102-109.

Dößelmann, B., M. Willmann, M. Steglich, B. Bunk, U. Nubel, S. Peter and R. A. Neher (2017). "Rapid and Consistent Evolution of Colistin Resistance in Extensively Drug-Resistant Pseudomonas aeruginosa during Morbidostat Culture." Antimicrob Agents Chemother 61(9): e00043-00017.

Drenkard, E. and F. M. Ausubel (2002). "Pseudomonas biofilm formation and antibiotic resistance are linked to phenotypic variation." Nature 416(6882): 740-743.

Dundar, D. and M. Otkun (2010). "In-Vitro Efficacy of Synergistic Antibiotic Combinations in Multidrug Resistant Pseudomonas Aeruginosa Strains." Yonsei Medical Journal 51(1): 111-116.

El-Halfawy, O. M. and M. A. Valvano (2015). "Antimicrobial Heteroresistance: an Emerging Field in Need of Clarity." Clinical Microbiology Reviews 28(1): 191-207.

El-Shouny, W., A. Al-Baidani and W. Hamza (2011). "Antimicrobial activity of pyocyanin produced by Pseudomonas aeruginosa isolated from surgical woundinfections." International Journal of Pharmacy and Medical Sciences 1(1): 01-07.

Evans, T. J. (2015). "Small colony variants of Pseudomonas aeruginosa in chronic bacterial infection of the lung in cystic fibrosis." Future Microbiology 10(2): 231-239.

Feoktistova, M., P. Geserick and M. Leverkus (2016). "Crystal Violet Assay for Determining Viability of Cultured Cells." Cold Spring Harbor Protocols 2016(4): pdb.prot087379.

Fernández, L., C. Álvarez-Ortega, I. Wiegand, J. Olivares, D. Kocíncová, J. S. Lam, J. L. Martínez and R. E. W. Hancock (2013). "Characterization of the Polymyxin B Resistome of Pseudomonas aeruginosa." Antimicrobial Agents and Chemotherapy 57(1): 110-119.

Fernández, L., W. J. Gooderham, M. Bains, J. B. McPhee, I. Wiegand and R. E. W. Hancock (2010). "Adaptive Resistance to the "Last Hope" Antibiotics Polymyxin B and Colistin in Pseudomonas aeruginosa Is Mediated by the Novel Two-Component Regulatory System ParR-ParS." Antimicrobial Agents and Chemotherapy 54(8): 3372-3382. 
Finlayson, E. A. and P. D. Brown (2011). "Comparison of antibiotic resistance and virulence factors in pigmented and non-pigmented Pseudomonas aeruginosa." West Indian Medical Journal 60(1): 24-32.

Fleming, D. and K. P. Rumbaugh (2017). "Approaches to Dispersing Medical Biofilms." Microorganisms 5(2): e15.

Flemming, H.-C., T. R. Neu and J. Wingender (2016). The perfect slime: microbial extracellular polymeric substances (EPS), IWA publishing.

Flemming, H.-K., J. Wingender, U. Szewzyk, P. Steinberg, S. A. Rice and S. Kjelleberg (2016). "Biofilms: an emergent form of bacterial life." Nature Reviews 14(94): 563-575.

Forge, A. and J. Schacht (2000). "Aminoglycoside Antibiotics." Audiology and Neurotology 5(1): 3-22.

Fournier, P.-E., M. Drancourt, P. Colson, J.-M. Rolain, B. L. Scola and D. Raoult (2013). "Modern clinical microbiology: new challenges and solutions." Nature Reviews Microbiology 11: 574-585.

Foweraker, J. E., C. R. Laughton, D. F. Brown and D. Bilton (2009). "Comparison of Methods To Test Antibiotic Combinations against Heterogeneous Populations of Multiresistant Pseudomonas aeruginosa from Patients with Acute Infective Exacerbations in Cystic Fibrosis." Antimicrobial Agents and Chemotherapy 53(11): 4809-4815.

Frangipani, E., V. I. Slaveykova, C. Reimmann and D. Haas (2008). "Adaptation of Aerobically Growing Pseudomonas aeruginosa to Copper Starvation." Journal of Bacteriology 190(20): 6706-6717.

Frank, L. H. and R. D. Demoss (1959). "On the biosynthesis of pyocyanine." Journal of Bacteriology 77(6): 776-782.

Frederiksen, B., T. Pressler, A. Hansen, C. Koch and N. Høiby (2006). "Effect of aerosolized rhDNase (Pulmozyme ${ }^{\circledR}$ ) on pulmonary colonization in patients with cystic fibrosis." Acta Pædiatrica 95(9): 1070-1074.

Friedman, L. and R. Kolter (2004). "Genes involved in matrix formation in Pseudomonas aeruginosa PA14 biofilms." Molecular Microbiology 51(3): 675-690.

Fux, C. A., P. Stoodley, L. Hall-Stoodley and J. W. Costerton (2003). "Bacterial biofilms: a diagnostic and therapeutic challenge." Expert Review of Anti-infective Therapy 1(4): 667-683.

Garey, K. W., Q. P. Vo, R. E. Lewis, W. Saengcharoen, M. T. LaRocco and V. H. Tam (2009). "Increased bacterial adherence and biomass in Pseudomonas aeruginosa bacteria exposed to clarithromycin." Diagnostic Microbiology and Infectious Disease 63(1): 81-86. 
Garrett, T. R., M. Bhakoo and Z. Zhang (2008). "Bacterial adhesion and biofilms on surfaces." Progress in Natural Science 18(9): 1049-1056.

Gellatly, S. L. and R. E. W. Hancock (2013). "Pseudomonas aeruginosa : new insights into pathogenesis and host defenses." Pathogens and Disease 67(3): 159173.

Gellatly, S. L., B. Needham, L. Madera, M. S. Trent and R. E. W. Hancock (2012). "The Pseudomonas aeruginosa PhoP-PhoQ Two-Component Regulatory System Is Induced upon Interaction with Epithelial Cells and Controls Cytotoxicity and Inflammation." Infection and Immunity 80(9): 3122-3131.

Gentleman, R. C., V. J. Carey, D. M. Bates, B. Bolstad, M. Dettling, S. Dudoit, B. Ellis, L. Gautier, Y. Ge, J. Gentry, K. Hornik, T. Hothorn, W. Huber, S. lacus, R. Irizarry, F. Leisch, C. Li, M. Maechler, A. J. Rossini, G. Sawitzki, C. Smith, G. Smyth, L. Tierney, J. Y. H. Yang and J. Zhang (2004). "Bioconductor: open software development for computational biology and bioinformatics." Genome Biology 5(10): R80.

Giacometti, A., O. Cirioni, F. Barchiesi, M. Fortuna and G. Scalise (1999). "In-vitro activity of cationic peptides alone and in combination with clinically used antimicrobial agents against Pseudomonas aeruginosa." Journal of Antimicrobial Chemotherapy 44(5): 641-645.

Gonzalez Moreno, M., A. Trampuz and M. Di Luca (2017). "Synergistic antibiotic activity against planktonic and biofilm-embedded Streptococcus agalactiae, Streptococcus pyogenes and Streptococcus oralis." Journal of Antimicrobial Chemotherapy 72(11): 3085-3092.

Gooderham, W. J., S. L. Gellatly, F. Sanschagrin, J. B. McPhee, M. Bains, C. Cosseau, R. C. Levesque and R. E. Hancock (2009). "The sensor kinase PhoQ mediates virulence in Pseudomonas aeruginosa." Microbiology 155(3): 699-711.

Gough, M., R. E. Hancock and N. M. Kelly (1996). "Antiendotoxin activity of cationic peptide antimicrobial agents." Infection and Immunity 64(12): 4922-4927.

Govan, J. R. and V. Deretic (1996). "Microbial pathogenesis in cystic fibrosis: mucoid Pseudomonas aeruginosa and Burkholderia cepacia." Microbiological Reviews 60(3): 539-574.

Grégoire, N., V. Aranzana-Climent, S. Magréault, S. Marchand and W. Couet (2017). "Clinical Pharmacokinetics and Pharmacodynamics of Colistin." Clinical Pharmacokinetics 56(12): 1441-1460.

Guilhen, C., C. Forestier and D. Balestrino (2017). "Biofilm dispersal: multiple elaborate strategies for dissemination of bacteria with unique properties." Molecular Microbiology 105(2): 188-210. 
Gunn, J. S., S. S. Ryan, J. C. Van Velkinburgh, R. K. Ernst and S. I. Miller (2000). "Genetic and Functional Analysis of a PmrA-PmrB-Regulated Locus Necessary for Lipopolysaccharide Modification, Antimicrobial Peptide Resistance, and Oral Virulence of Salmonella enterica Serovar Typhimurium." Infection and Immunity 68(11): 6139-6146.

Gutu, A. D., N. Sgambati, P. Strasbourger, M. K. Brannon, M. A. Jacobs, E. Haugen, R. K. Kaul, H. K. Johansen, N. Hoiby and S. M. Moskowitz (2013). "Polymyxin resistance of Pseudomonas aeruginosa phoQ mutants is dependent on additional two-component regulatory systems." Antimicrobial agents and chemotherapy 57(5): 2204-2215.

Ha, D. G. and G. A. O'Toole (2015). "c-di-GMP and its Effects on Biofilm Formation and Dispersion: a Pseudomonas Aeruginosa Review." Microbiology Spectrum 3(2): Mb-0003-2014.

Haney, E., M. Trimble, J. Cheng, Q. Vallé and R. Hancock (2018). "Critical Assessment of Methods to Quantify Biofilm Growth and Evaluate Antibiofilm Activity of Host Defence Peptides." Biomolecules 8(2): 29.

Harmsen, M., L. Yang, S. J. Pamp and T. Tolker-Nielsen (2010). "An update on Pseudomonas aeruginosa biofilm formation, tolerance, and dispersal." FEMS Immunology \& Medical Microbiology 59(3): 253-268.

Häußler, S. (2004). "Biofilm formation by the small colony variant phenotype of Pseudomonas aeruginosa." Environmental Microbiology 6(6): 546-551.

Häußler, S., B. Tummler, H. Weissbrodt, M. Rohde and I. Steinmetz (1999). "Smallcolony variants of Pseudomonas aeruginosa in cystic fibrosis." Clinical Infectious Diseases 29(3): 621-625.

Hazan, R., Y.-A. Que, D. Maura and L. G. Rahme (2012). "A method for high throughput determination of viable bacteria cell counts in 96-well plates." BMC Microbiology 12: 259-259.

Hengzhuang, W., H. Wu, O. Ciofu, Z. Song and N. Høiby (2011). "Pharmacokinetics/Pharmacodynamics of Colistin and Imipenem on Mucoid and Nonmucoid Pseudomonas aeruginosa Biofilms." Antimicrobial Agents and Chemotherapy 55(9): 4469-4474.

Hengzhuang, W., H. Wu, O. Ciofu, Z. Song and N. Høiby (2012). "In Vivo Pharmacokinetics/Pharmacodynamics of Colistin and Imipenem in Pseudomonas aeruginosa Biofilm Infection." Antimicrobial Agents and Chemotherapy 56(5): 26832690.

Hermes, D. M., C. Pormann Pitt, L. Lutz, A. B. Teixeira, V. B. Ribeiro, B. Netto, A. F. Martins, A. P. Zavascki and A. L. Barth (2013). "Evaluation of heteroresistance to polymyxin B among carbapenem-susceptible and -resistant Pseudomonas aeruginosa." Journal of Medical Microbiology 62(8): 1184-1189. 
Herrmann, G., L. Yang, H. Wu, Z. Song, H. Wang, N. Høiby, M. Ulrich, S. Molin, J. Riethmüller and G. Döring (2010). "Colistin-tobramycin combinations are superior to monotherapy concerning the killing of biofilm Pseudomonas aeruginosa." The Journal of infectious diseases 202(10): 1585-1592.

Heydorn, A., B. K. Ersboll, M. Hentzer, M. R. Parsek, M. Givskov and S. Molin (2000). "Experimental reproducibility in flow-chamber biofilms." Microbiology 146 (10): 24092415.

Heydorn, A., A. T. Nielsen, M. Hentzer, C. Sternberg, M. Givskov, B. K. Ersbøll and S. Molin (2000). "Quantification of biofilm structures by the novel computer program COMSTAT." Microbiology 146(10): 2395-2407.

Hill, D., B. Rose, A. Pajkos, M. Robinson, P. Bye, S. Bell, M. Elkins, B. Thompson, C. MacLeod, S. D. Aaron and C. Harbour (2005). "Antibiotic Susceptibilities of Pseudomonas aeruginosa Isolates Derived from Patients with Cystic Fibrosis under Aerobic, Anaerobic, and Biofilm Conditions." Journal of Clinical Microbiology 43(10): 5085-5090.

Hogardt, M. and J. Heesemann (2010). "Adaptation of Pseudomonas aeruginosa during persistence in the cystic fibrosis lung." International Journal of Medical Microbiology 300(8): 557-562.

Hogardt, M. and J. Heesemann (2013). "Microevolution of Pseudomonas aeruginosa to a chronic pathogen of the cystic fibrosis lung." Curr Top Microbiol Immunol 358: 91 118.

Høiby, N., T. Bjarnsholt, M. Givskov, S. Molin and O. Ciofu (2010). "Antibiotic resistance of bacterial biofilms." International Journal of Antimicrobial Agents 35(4): 322-332.

Høiby, N., T. Bjarnsholt, C. Moser, G. L. Bassi, T. Coenye, G. Donelli, L. HallStoodley, V. Holá, C. Imbert, K. Kirketerp-Møller, D. Lebeaux, A. Oliver, A. J. Ullmann and C. Williams (2014). "ESCMID guideline for the diagnosis and treatment of biofilm infections 2014." Clinical Microbiology and Infection 21: S1-S25.

Høiby, N., T. Bjarnsholt, C. Moser, G. L. Bassi, T. Coenye, G. Donelli, L. HallStoodley, V. Holá, C. Imbert, K. Kirketerp-Møller, D. Lebeaux, A. Oliver, A. J. Ullmann and C. Williams (2015). "ESCMID guideline for the diagnosis and treatment of biofilm infections 2014." Clinical Microbiology and Infection 21: S1-S25.

Høiby, N., H. Krogh Johansen, C. Moser, Z. Song, O. Ciofu and A. Kharazmi (2001). "Pseudomonas aeruginosa and the in-vitro and in-vivo biofilm mode of growth." Microbes and Infection 3(1): 23-35. 
Howlin, R. P., K. Cathie, L. Hall-Stoodley, V. Cornelius, C. Duignan, R. N. Allan, B. O. Fernandez, N. Barraud, K. D. Bruce, J. Jefferies, M. Kelso, S. Kjelleberg, S. A. Rice, G. B. Rogers, S. Pink, C. Smith, P. S. Sukhtankar, R. Salib, J. Legg, M. Carroll, T. Daniels, M. Feelisch, P. Stoodley, S. C. Clarke, G. Connett, S. N. Faust and J. S. Webb (2017). "Low-Dose Nitric Oxide as Targeted Anti-biofilm Adjunctive Therapy to Treat Chronic Pseudomonas aeruginosa Infection in Cystic Fibrosis." Molecular Therapy 25(9): 2104-2116.

Hu, Q., H. Peng and X. Rao (2016). "Molecular Events for Promotion of Vancomycin Resistance in Vancomycin Intermediate Staphylococcus aureus." Frontiers in Microbiology 7: 1601.

Huttner, A., S. Harbarth, J. Carlet, S. Cosgrove, H. Goossens, A. Holmes, V. Jarlier, A. Voss and D. Pittet (2013). "Antimicrobial resistance: a global view from the 2013 World Healthcare-Associated Infections Forum." Antimicrobial Resistance and Infection Control 2(1): 31.

Izadpanah, M. and H. Khalili (2015). "Antibiotic regimens for treatment of infections due to multidrug-resistant Gram-negative pathogens: An evidence-based literature review." Journal of Research in Pharmacy Practice 4(3): 105-114.

Jain, K., S. Parida, N. Mangwani, H. R. Dash and S. Das (2013). "Isolation and characterization of biofilm-forming bacteria and associated extracellular polymeric substances from oral cavity." Annals of Microbiology 63(4): 1553-1562.

Jakobsen, T., T. Tolker-Nielsen and M. Givskov (2017). "Bacterial Biofilm Control by Perturbation of Bacterial Signaling Processes." International Journal of Molecular Sciences 18(9): 1970.

Jansen, G., N. Mahrt, L. Tueffers, C. Barbosa, M. Harjes, G. Adolph, A. Friedrichs, A. Krenz-Weinreich, P. Rosenstiel and H. Schulenburg (2016). "Association between clinical antibiotic resistance and susceptibility of Pseudomonas in the cystic fibrosis lung." Evolution, Medicine, and Public Health 2016(1): 182-194.

Jenkins, S. G. and A. N. Schuetz (2012). "Current Concepts in Laboratory Testing to Guide Antimicrobial Therapy." Mayo Clinic Proceedings 87(3): 290-308.

Jochumsen, N., R. L. Marvig, S. Damkiær, R. L. Jensen, W. Paulander, S. Molin, L. Jelsbak and A. Folkesson (2016). "The evolution of antimicrobial peptide resistance in Pseudomonas aeruginosa is shaped by strong epistatic interactions." Nature Communications 7: 13002.

Johnson, M. D., R. L. Nation and J. Li (2017). Mechanism of the Antibacterial Activity and Resistance of Polymyxins. Antimicrobial Drug Resistance: Mechanisms of Drug Resistance, Volume 1. D. L. Mayers, J. D. Sobel, M. Ouellette, K. S. Kaye and D. Marchaim. Cham, Springer International Publishing: 333-344.

Kalan, L. and G. D. Wright (2011). "Antibiotic adjuvants: multicomponent anti-infective strategies." Expert Reviews in Molecular Medicine 13: e5. 
Kaplan, J. (2010). "Biofilm Dispersal: Mechanisms, Clinical Implications, and Potential Therapeutic Uses." J Dent Res 89(3): 205-218.

Kapoor, P. and P. Murphy (2018). "Combination antibiotics against Pseudomonas aeruginosa, representing common and rare cystic fibrosis strains from different Irish clinics." Heliyon 4(3): e00562.

Kessler, E. and M. Safrin (2014). Elastinolytic and Proteolytic Enzymes.

Pseudomonas Methods and Protocols. A. Filloux and J.-L. Ramos. New York, NY, Springer New York: 135-169.

Khlebnikov, A., Ø. Risa, T. Skaug, T. A. Carrier and J. D. Keasling (2000). "Regulatable Arabinose-Inducible Gene Expression System with Consistent Control in All Cells of a Culture." Journal of Bacteriology 182(24): 7029-7034.

Kim, H. J. and S. Jang (2017). "Optimization of a resazurin-based microplate assay for large-scale compound screenings against Klebsiella pneumoniae." 3 Biotech 8(1): 3.

Kim, J.-S., N. Chowdhury, R. Yamasaki and T. K. Wood (2018). "Viable But NonCulturable and Persistence Describe the Same Bacterial Stress State." Environmental Microbiology: Accepted Article.

Kim, S.-K. and J.-H. Lee (2016). "Biofilm dispersion in Pseudomonas aeruginosa." Journal of Microbiology 54(2): 71-85.

Kirisits, M. J., L. Prost, M. Starkey and M. R. Parsek (2005). "Characterization of Colony Morphology Variants Isolated from Pseudomonas aeruginosa Biofilms." Applied and Environmental Microbiology 71(8): 4809-4821.

Kirov, S. M., J. S. Webb, Y. Che, D. W. Reid, J. K. Woo, S. A. Rice and S. Kjelleberg (2007). "Biofilm differentiation and dispersal in mucoid Pseudomonas aeruginosa isolates from patients with cystic fibrosis." Microbiology 153(10): 3264-3274.

Klausen, M., A. Heydorn, P. Ragas, L. Lambertsen, A. Aaes-Jørgensen, S. Molin and T. Tolker-Nielsen (2003). "Biofilm formation by Pseudomonas aeruginosa wild type, flagella and type IV pili mutants." Molecular Microbiology 48(6): 1511-1524.

Koch, A. K., O. Käppeli, A. Fiechter and J. Reiser (1991). "Hydrocarbon assimilation and biosurfactant production in Pseudomonas aeruginosa mutants." Journal of Bacteriology 173(13): 4212-4219.

Koeth, L. M., A. King, H. Knight, J. May, L. A. Miller, I. Phillips and J. A. Poupard (2000). "Comparison of cation-adjusted Mueller-Hinton broth with Iso-Sensitest broth for the NCCLS broth microdilution method." Journal of Antimicrobial Chemotherapy 46(3): 369-376.

Konrat, K., I. Schwebke, M. Laue, C. Dittmann, K. Levin, R. Andrich, M. Arvand and C. Schaudinn (2016). "The Bead Assay for Biofilms: A Quick, Easy and Robust Method for Testing Disinfectants." PLOS ONE 11(6): e0157663. 
Kostakioti, M., M. Hadjifrangiskou and S. J. Hultgren (2013). "Bacterial Biofilms: Development, Dispersal, and Therapeutic Strategies in the Dawn of the Postantibiotic Era." Cold Spring Harbor Perspectives in Medicine 3(4): a010306.

Krahn, T., C. Gilmour, J. Tilak, S. Fraud, N. Kerr, C. H.-F. Lau and K. Poole (2012). "Determinants of Intrinsic Aminoglycoside Resistance in Pseudomonas aeruginosa." Antimicrobial Agents and Chemotherapy 56(11): 5591-5602.

Lade, H., D. Paul and J. H. Kweon (2014). "N-Acyl Homoserine Lactone-Mediated Quorum Sensing with Special Reference to Use of Quorum Quenching Bacteria in Membrane Biofouling Control." BioMed Research International 2014: 162584.

Landini, P., D. Antoniani, J. G. Burgess and R. Nijland (2010). "Molecular mechanisms of compounds affecting bacterial biofilm formation and dispersal." Applied Microbiology and Biotechnology 86(3): 813-823.

Larimer, C., E. Winder, R. Jeters, M. Prowant, I. Nettleship, R. S. Addleman and G. T. Bonheyo (2016). "A method for rapid quantitative assessment of biofilms with biomolecular staining and image analysis." Analytical and Bioanalytical Chemistry 408: 999-1008.

Lebeaux, D., J.-M. Ghigo and C. Beloin (2014). "Biofilm-Related Infections: Bridging the Gap between Clinical Management and Fundamental Aspects of Recalcitrance toward Antibiotics." Microbiology and Molecular Biology Reviews : MMBR 78(3): 510543.

Lee, J.-Y., Y. K. Park, E. S. Chung, I. Y. Na and K. S. Ko (2016). "Evolved resistance to colistin and its loss due to genetic reversion in Pseudomonas aeruginosa."

Scientific Reports 6: 25543.

Lee, J. and L. Zhang (2015). "The hierarchy quorum sensing network in Pseudomonas aeruginosa." Protein \& Cell 6(1): 26-41.

Lee, J. Y., I. Y. Na, Y. K. Park and K. S. Ko (2014). "Genomic variations between colistin-susceptible and -resistant Pseudomonas aeruginosa clinical isolates and their effects on colistin resistance." Journal of Antimicrobial Chemotherapy 69(5): 12481256.

Lee, L. S., M. Kinzig-Schippers, A. N. Nafziger, L. Ma, F. Sörgel, R. N. Jones, G. L. Drusano and J. S. Bertino (2010). "Comparison of 30-min and 3-h infusion regimens for imipenem/cilastatin and for meropenem evaluated by Monte Carlo simulation." Diagnostic Microbiology and Infectious Disease 68(3): 251-258.

Lewenza, S. (2013). "Extracellular DNA-induced antimicrobial peptide resistance mechanisms in Pseudomonas aeruginosa." Frontiers in Microbiology 4: 21.

Lewis, K. (2008). Multidrug tolerance of biofilms and persister cells. Bacterial Biofilms, Springer: 107-131. 
Lewis, K., A. Spoering, N. Kaldalu, I. Keren and D. Shah (2005). "Persisters: specialized cells responsible for biofilm tolerance to antimicrobial agents." Biofilms, Infection, and Antimicrobial Therapy. Boca Raton, London, New York, Singapore: Taylor \& Francis: 241-256.

Li, H., X. Li, Z. Wang, Y. Fu, Q. Ai, Y. Dong and J. Yu (2015). "Autoinducer-2 regulates Pseudomonas aeruginosa PAO1 biofilm formation and virulence production in a dose-dependent manner." BMC Microbiology 15: 192.

Li, J., K. Coulthard, R. Milne, R. L. Nation, S. Conway, D. Peckham, C. Etherington and J. Turnidge (2003). "Steady-state pharmacokinetics of intravenous colistin methanesulphonate in patients with cystic fibrosis." Journal of Antimicrobial Chemotherapy 52(6): 987-992.

Li, J., C. R. Rayner, R. L. Nation, R. J. Owen, D. Spelman, K. E. Tan and L. Liolios (2006). "Heteroresistance to colistin in multidrug-resistant Acinetobacter baumannii." Antimicrobial agents and chemotherapy 50(9): 2946-2950.

Li, Y., O. E. Petrova, S. Su, G. W. Lau, W. Panmanee, R. Na, D. J. Hassett, D. G. Davies and K. Sauer (2014). "BdIA, DipA and Induced Dispersion Contribute to Acute Virulence and Chronic Persistence of Pseudomonas aeruginosa." PLOS Pathogens 10(6): e1004168.

Lim, L. M., N. Ly, D. Anderson, J. C. Yang, L. Macander, A. Jarkowski, A. Forrest, J. B. Bulitta and B. T. Tsuji (2010). "Resurgence of Colistin: A Review of Resistance, Toxicity, Pharmacodynamics, and Dosing." Pharmacotherapy: The Journal of Human Pharmacology and Drug Therapy 30(12): 1279-1291.

Lister, P. D., D. J. Wolter and N. D. Hanson (2009). "Antibacterial-Resistant Pseudomonas aeruginosa: Clinical Impact and Complex Regulation of Chromosomally Encoded Resistance Mechanisms." Clinical Microbiology Reviews 22(4): 582-610.

Liu, Y.-Y., C. E. Chandler, L. M. Leung, C. L. McElheny, R. T. Mettus, R. M. Q. Shanks, J.-H. Liu, D. R. Goodlett, R. K. Ernst and Y. Doi (2017). "Structural Modification of Lipopolysaccharide Conferred by mcr-1 in Gram-Negative ESKAPE Pathogens." Antimicrobial Agents and Chemotherapy 61(6).

Liu, Y.-Y., Y. Wang, T. R. Walsh, L.-X. Yi, R. Zhang, J. Spencer, Y. Doi, G. Tian, B. Dong, X. Huang, L.-F. Yu, D. Gu, H. Ren, X. Chen, L. Lv, D. He, H. Zhou, Z. Liang, J.$\mathrm{H}$. Liu and J. Shen (2016). "Emergence of plasmid-mediated colistin resistance mechanism mcr-1 in animals and human beings in China: a microbiological and molecular biological study." The Lancet Infectious Diseases 16(2): 161-168.

Livermore, D. M. (2002). "Multiple Mechanisms of Antimicrobial Resistance in Pseudomonas aeruginosa: Our Worst Nightmare?" Clinical Infectious Diseases 34(5): 634-640. 
Lo Sciuto, A. and F. Imperi (2018). "Aminoarabinosylation of Lipid A Is Critical for the Development of Colistin Resistance in Pseudomonas aeruginosa." Antimicrobial Agents and Chemotherapy 62(3).

Louie, A., W. Liu, S. Fikes, D. Brown and G. L. Drusano (2013). "Impact of meropenem in combination with tobramycin in a murine model of Pseudomonas aeruginosa pneumonia." Antimicrobial agents and chemotherapy 57(6): 2788-2792.

Macia, M., E. Rojo-Molinero and A. Oliver (2014). "Antimicrobial susceptibility testing in biofilm-growing bacteria." Clinical Microbiology and Infection 20(10): 981-990.

Maldonado, R. F., I. Sá-Correia and M. A. Valvano (2016). "Lipopolysaccharide modification in Gram-negative bacteria during chronic infection." FEMS Microbiology Reviews 40(4): 480-493.

Malone, M., D. M. Goeres, I. Gosbell, K. Vickery, S. Jensen and P. Stoodley (2017). "Approaches to biofilm-associated infections: the need for standardized and relevant biofilm methods for clinical applications." Expert Review of Anti-infective Therapy 15(2): 147-156.

Marina, A., C. Mott, A. Auyzenberg, W. A. Hendrickson and C. D. Waldburger (2001). "Structural and mutational analysis of the PhoQ histidine kinase catalytic domain. Insight into the reaction mechanism." The Journal of biological chemistry 276(44): 41182-41190.

Martínez, E. and J. Campos-Gómez (2016). "Pf Filamentous Phage Requires UvrD for Replication in Pseudomonas aeruginosa." mSphere 1(1): e00104-00115.

Martínez, J. L. and F. Rojo (2011). "Metabolic regulation of antibiotic resistance." FEMS microbiology reviews 35(5): 768-789.

Martis, N., S. Leroy and V. Blanc (2014). "Colistin in multi-drug resistant Pseudomonas aeruginosa blood-stream infections." Journal of Infection 69(1): 1-12.

McDougald, D., S. A. Rice, N. Barraud, P. D. Steinberg and S. Kjelleberg (2011). "Should we stay or should we go: mechanisms and ecological consequences for biofilm dispersal." Nature Reviews Microbiology 10(1): 39-50.

McPhee, J. B., M. Bains, G. Winsor, S. Lewenza, A. Kwasnicka, M. D. Brazas, F. S. L. Brinkman and R. E. W. Hancock (2006). "Contribution of the PhoP-PhoQ and PmrA-PmrB Two-Component Regulatory Systems to $\mathrm{Mg}^{2+}$-Induced Gene Regulation in Pseudomonas aeruginosa." Journal of Bacteriology 188(11): 3995-4006.

Mdluli, K. E., P. R. Witte, T. Kline, A. W. Barb, A. L. Erwin, B. E. Mansfield, A. L. McClerren, M. C. Pirrung, L. N. Tumey, P. Warrener, C. R. H. Raetz and C. K. Stover (2006). "Molecular Validation of LpxC as an Antibacterial Drug Target in Pseudomonas aeruginosa." Antimicrobial Agents and Chemotherapy 50(6): 21782184. 
Miller, A. K., M. K. Brannon, L. Stevens, H. K. Johansen, S. E. Selgrade, S. I. Miller, N. Høiby and S. M. Moskowitz (2011). "PhoQ Mutations Promote Lipid A Modification and Polymyxin Resistance of Pseudomonas aeruginosa Found in Colistin-Treated Cystic Fibrosis Patients." Antimicrobial Agents and Chemotherapy 55(12): 5761-5769.

Miller, M. B. and B. L. Bassler (2001). "Quorum Sensing in Bacteria." Annual Review of Microbiology 55(1): 165-199.

Moradali, M. F., S. Ghods and B. H. A. Rehm (2017). "Pseudomonas aeruginosa Lifestyle: A Paradigm for Adaptation, Survival, and Persistence." Frontiers in Cellular and Infection Microbiology 7(39).

Morita, Y., J. Tomida and Y. Kawamura (2013). "Responses of Pseudomonas aeruginosa to antimicrobials." Frontiers in Microbiology 4: 422.

Moskowitz, S. M., M. K. Brannon, N. Dasgupta, M. Pier, N. Sgambati, A. K. Miller, S. E. Selgrade, S. I. Miller, M. Denton, S. P. Conway, H. K. Johansen and N. Høiby (2012). "PmrB Mutations Promote Polymyxin Resistance of Pseudomonas aeruginosa Isolated from Colistin-Treated Cystic Fibrosis Patients." Antimicrobial Agents and Chemotherapy 56(2): 1019-1030.

Moskowitz, S. M., R. K. Ernst and S. I. Miller (2004). "PmrAB, a Two-Component Regulatory System of Pseudomonas aeruginosa That Modulates Resistance to Cationic Antimicrobial Peptides and Addition of Aminoarabinose to Lipid A." Journal of Bacteriology 186(2): 575-579.

Moskowitz, S. M., J. M. Foster, J. Emerson and J. L. Burns (2004). "Clinically Feasible Biofilm Susceptibility Assay for Isolates of Pseudomonas aeruginosa from Patients with Cystic Fibrosis." Journal of Clinical Microbiology 42(5): 1915-1922.

Mowat, E., S. Paterson, J. L. Fothergill, E. A. Wright, M. J. Ledson, M. J. Walshaw, M. A. Brockhurst and C. Winstanley (2011). "Pseudomonas aeruginosa Population Diversity and Turnover in Cystic Fibrosis Chronic Infections." American Journal of Respiratory and Critical Care Medicine 183(12): 1674-1679.

Moyá, B., A. Beceiro, G. Cabot, C. Juan, L. Zamorano, S. Alberti and A. Oliver (2012). "Pan- $\beta$-Lactam Resistance Development in Pseudomonas aeruginosa Clinical Strains: Molecular Mechanisms, Penicillin-Binding Protein Profiles, and Binding Affinities." Antimicrobial Agents and Chemotherapy 56(9): 4771-4778.

Mueller, J. H. and J. Hinton (1941). "A Protein-Free Medium for Primary Isolation of the Gonococcus and Meningococcus." Proceedings of the Society for Experimental Biology and Medicine 48(1): 330-333.

Mulcahy, H., L. Charron-Mazenod and S. Lewenza (2008). "Extracellular DNA Chelates Cations and Induces Antibiotic Resistance in Pseudomonas aeruginosa Biofilms." PLOS Pathogens 4(11): e1000213. 
Mustafa, M.-H., H. Chalhoub, O. Denis, A. Deplano, A. Vergison, H. RodriguezVillalobos, M. M. Tunney, J. S. Elborn, B. C. Kahl, H. Traore, F. Vanderbist, P. M. Tulkens and F. Van Bambeke (2016). "Antimicrobial Susceptibility of Pseudomonas aeruginosa Isolated from Cystic Fibrosis Patients in Northern Europe." Antimicrobial Agents and Chemotherapy 60(11): 6735-6741.

Nadell, C. D., D. Ricaurte, J. Yan, K. Drescher and B. L. Bassler (2017). "Flow environment and matrix structure interact to determine spatial competition in Pseudomonas aeruginosa biofilms." eLife 6: e21855.

Ni, L., S. Yang, R. Zhang, Z. Jin, H. Chen, J. C. Conrad and F. Jin (2016). "Bacteria differently deploy type-IV pili on surfaces to adapt to nutrient availability." Npj Biofilms And Microbiomes 2: 15029.

Nickel, J. C., I. Ruseska, J. B. Wright and J. W. Costerton (1985). "Tobramycin resistance of Pseudomonas aeruginosa cells growing as a biofilm on urinary catheter material." Antimicrobial Agents and Chemotherapy 27(4): 619-624.

O'Toole, G. A. (2011). "Microtiter Dish Biofilm Formation Assay." Journal of Visualized Experiments : JoVE(47): 2437.

O'Neill, J. (2014). "Antimicrobial resistance: tackling a crisis for the health and wealth of nations." The Review on Antimicrobial Resistance 20.

Okamoto, K., N. Gotoh and T. Nishino (2002). "Extrusion of Penem Antibiotics by Multicomponent Efflux Systems MexAB-OprM, MexCD-OprJ, and MexXY-OprM of Pseudomonas aeruginosa." Antimicrobial Agents and Chemotherapy 46(8): 26962699.

Olaitan, A. O., S. Morand and J.-M. Rolain (2014). "Mechanisms of polymyxin resistance: acquired and intrinsic resistance in bacteria." Frontiers in Microbiology 5 : 643.

Ortwine, J. K., B. J. Werth, G. Sakoulas and M. J. Rybak (2013). "Reduced glycopeptide and lipopeptide susceptibility in Staphylococcus aureus and the "seesaw effect": Taking advantage of the back door left open?" Drug Resistance Updates 16(3): 73-79.

Palmer, J., S. Flint and J. Brooks (2007). "Bacterial cell attachment, the beginning of a biofilm." Journal of Industrial Microbiology \& Biotechnology 34(9): 577-588.

Pamp, S. J., M. Gjermansen, H. K. Johansen and T. Tolker-Nielsen (2008). "Tolerance to the antimicrobial peptide colistin in Pseudomonas aeruginosa biofilms is linked to metabolically active cells, and depends on the pmr and mexAB-oprM genes." Molecular Microbiology 68(1): 223-240.

Pedersen, S. S., T. Pressler, T. Jensen, V. T. Rosdahl, M. W. Bentzon, N. Høiby and C. Koch (1987). "Combined imipenem/cilastatin and tobramycin therapy of multiresistant Pseudomonas aeruginosa in cystic fibrosis." J Antimicrob Chemother 19(1): 101-107. 
Pena-Miller, R., D. Laehnemann, G. Jansen, A. Fuentes-Hernandez, P. Rosenstiel, H. Schulenburg and R. Beardmore (2013). "When the Most Potent Combination of Antibiotics Selects for the Greatest Bacterial Load: The Smile-Frown Transition." PLoS Biology 11(4): e1001540.

Petrova, O. E., K. E. Cherny and K. Sauer (2014). "The Pseudomonas aeruginosa Diguanylate Cyclase GcbA, a Homolog of $P$. fluorescens GcbA, Promotes Initial Attachment to Surfaces, but Not Biofilm Formation, via Regulation of Motility." Journal of Bacteriology 196(15): 2827-2841.

Petrova, O. E. and K. Sauer (2012). "PAS Domain Residues and Prosthetic Group Involved in BdlA-Dependent Dispersion Response by Pseudomonas aeruginosa Biofilms." Journal of Bacteriology 194(21): 5817-5828.

Poole, K. (2005). "Aminoglycoside Resistance in Pseudomonas aeruginosa." Antimicrobial Agents and Chemotherapy 49(2): 479-487.

Prost, L. R., M. E. Daley, V. Le Sage, M. W. Bader, H. Le Moual, R. E. Klevit and S. I. Miller (2007). "Activation of the Bacterial Sensor Kinase PhoQ by Acidic pH." Molecular Cell 26(2): 165-174.

Pu, M. and T. K. Wood (2010). "Tyrosine phosphatase TpbA controls rugose colony formation in Pseudomonas aeruginosa by dephosphorylating diguanylate cyclase TpbB." Biochemical and Biophysical Research Communications 402(2): 351-355.

Rakhimova, E., A. Munder, L. Wiehlmann, F. Bredenbruch and B. Tümmler (2008). "Fitness of Isogenic Colony Morphology Variants of Pseudomonas aeruginosa in Murine Airway Infection." PLoS ONE 3(2): e1685.

Rasamiravaka, T., Q. Labtani, P. Duez and M. El Jaziri (2015). "The Formation of Biofilms by Pseudomonas aeruginosa: A Review of the Natural and Synthetic Compounds Interfering with Control Mechanisms." BioMed Research International 2015: 17.

Rau, M. H., S. K. Hansen, H. K. Johansen, L. E. Thomsen, C. T. Workman, K. F. Nielsen, L. Jelsbak, N. Høiby, L. Yang and S. Molin (2010). "Early adaptive developments of Pseudomonas aeruginosa after the transition from life in the environment to persistent colonization in the airways of human cystic fibrosis hosts." Environmental Microbiology 12(6): 1643-1658.

Regenbogen, B., M. Willmann, M. Steglich, B. Bunk, U. Nübel, S. Peter and R. A. Neher (2017). "Rapid and consistent evolution of colistin resistance in Pseudomonas aeruginosa during morbidostat culture." Antimicrobial agents and chemotherapy 61(9): e00043-00017.

Rello, J. (2007). "Importance of appropriate initial antibiotic therapy and de-escalation in the treatment of nosocomial pneumonia." European Respiratory Review 16(103): 33-39. 
Ren, H., J. Wu, A. Colletta, M. E. Meyerhoff and C. Xi (2016). "Efficient Eradication of Mature Pseudomonas aeruginosa Biofilm via Controlled Delivery of Nitric Oxide Combined with Antimicrobial Peptide and Antibiotics." Frontiers in Microbiology 7: 1260.

Ren, H., J. Wu, A. Colletta, M. E. Meyerhoff and C. Xi (2016). "Efficient Eradication of Mature Pseudomonas aeruginosa Biofilm via Controlled Delivery of Nitric Oxide Combined with Antimicrobial Peptide and Antibiotics." Frontiers in Microbiology 7: e1260.

Rodrigue, A., Y. Quentin, A. Lazdunski, V. Méjean and M. Foglino (2000). "Cell signalling by oligosaccharides. Two-component systems in Pseudomonas aeruginosa: why so many?" Trends in Microbiology 8(11): 498-504.

Rogers, S. A., R. W. Huigens, J. Cavanagh and C. Melander (2010). "Synergistic Effects between Conventional Antibiotics and 2-Aminoimidazole-Derived Antibiofilm Agents." Antimicrobial Agents and Chemotherapy 54(5): 2112-2118.

Roizman, D., C. Vidaillac, M. Givskov and L. Yang (2017). "In-Vitro Evaluation of Biofilm Dispersal as a Therapeutic Strategy To Restore Antimicrobial Efficacy." Antimicrobial agents and chemotherapy 61(10): e01088-01017.

Sakuragi, Y. and R. Kolter (2007). "Quorum-Sensing Regulation of the Biofilm Matrix Genes (pel) of Pseudomonas aeruginosa." Journal of Bacteriology 189(14): 53835386 .

Sánchez-Gómez, S., R. Ferrer-Espada, P. S. Stewart, B. Pitts, K. Lohner and G. Martínez de Tejada (2015). "Antimicrobial activity of synthetic cationic peptides and lipopeptides derived from human lactoferricin against Pseudomonas aeruginosa planktonic cultures and biofilms." BMC Microbiology 15: 137.

Sandberg, M. E., D. Schellmann, G. Brunhofer, T. Erker, I. Busygin, R. Leino, P. M. Vuorela and A. Fallarero (2009). "Pros and cons of using resazurin staining for quantification of viable Staphylococcus aureus biofilms in a screening assay." Journal of Microbiological Methods 78(1): 104-106.

Schniederjans, M., M. Koska and S. Häussler (2017). "Transcriptional and Mutational Profiling of an Aminoglycoside-Resistant Pseudomonas aeruginosa Small-Colony Variant." Antimicrobial Agents and Chemotherapy 61(11).

Schurek, K. N., A. K. Marr, P. K. Taylor, I. Wiegand, L. Semenec, B. K. Khaira and R. E. W. Hancock (2008). "Novel Genetic Determinants of Low-Level Aminoglycoside Resistance in Pseudomonas aeruginosa." Antimicrobial Agents and Chemotherapy 52(12): 4213-4219.

Schwalbe, R., L. Steele-Moore and A. C. Goodwin (2007). Antimicrobial susceptibility testing protocols, Crc Press. 
Scott, C., M. E. Hilton, C. W. Coppin, R. J. Russell, J. G. Oakeshott and T. D. Sutherland (2007). "A global response to sulfur starvation in Pseudomonas putida and its relationship to the expression of low sulfur content proteins." FEMS Microbiology Letters 267(2): 184-193.

Shafiei, M., A. Abdi Ali, F. Shahcheraghi, A. Saboora and K. Akbari Noghabi (2014). "Eradication of Pseudomonas aeruginosa Biofilms Using the Combination of n-butanolic Cyclamen coum Extract and Ciprofloxacin." Jundishapur Journal of Microbiology 7(2): e14358.

Sheraton, M. V., K. H. Yam, C. H. Tan, H. S. Oh, E. Mancini, L. Yang, S. A. Rice and P. M. A. Sloot (2018). Mesoscopic Energy Minimization Drives Pseudomonas aeruginosa Biofilm Morphologies and Consequent Stratification of Antibiotic Activity Based on Cell Metabolism.

Shrout, J. D., D. L. Chopp, C. L. Just, M. Hentzer, M. Givskov and M. R. Parsek (2006). "The impact of quorum sensing and swarming motility on Pseudomonas aeruginosa biofilm formation is nutritionally conditional." Molecular Microbiology 62(5): 1264-1277.

Sieradzki, K. and A. Tomasz (1997). "Inhibition of cell wall turnover and autolysis by vancomycin in a highly vancomycin-resistant mutant of Staphylococcus aureus." Journal of Bacteriology 179(8): 2557-2566.

Sintim, H. O., J. A. Smith, J. Wang, S. Nakayama and L. Yan (2010). "Paradigm shift in discovering next-generation anti-infective agents: targeting quorum sensing, c-diGMP signaling and biofilm formation in bacteria with small molecules." Future Medicinal Chemistry 2(6): 1005-1035.

Smith, R. and J. Coast (2012). "The economic burden of antimicrobial resistance: why it is more serious than current studies suggest."

Spoering, A. L. and K. Lewis (2001). "Biofilms and Planktonic Cells of Pseudomonas aeruginosa Have Similar Resistance to Killing by Antimicrobials." Journal of Bacteriology 183(23): 6746-6751.

Starkey, M., J. H. Hickman, L. Ma, N. Zhang, S. De Long, A. Hinz, S. Palacios, C. Manoil, M. J. Kirisits, T. D. Starner, D. J. Wozniak, C. S. Harwood and M. R. Parsek (2009). "Pseudomonas aeruginosa Rugose Small-Colony Variants Have Adaptations That Likely Promote Persistence in the Cystic Fibrosis Lung." Journal of Bacteriology 191(11): 3492-3503.

Steed, M. E., B. J. Werth, C. E. Ireland and M. J. Rybak (2012). "Evaluation of the Novel Combination of High-Dose Daptomycin plus Trimethoprim-Sulfamethoxazole against Daptomycin-Nonsusceptible Methicillin-Resistant Staphylococcus aureus Using an In Vitro Pharmacokinetic/Pharmacodynamic Model of Simulated Endocardial Vegetations." Antimicrobial Agents and Chemotherapy 56(11): 57095714. 
Sternberg, C., T. Bjarnsholt and M. Shirtliff (2014). Methods for Dynamic Investigations of Surface-Attached In Vitro Bacterial and Fungal Biofilms. Microbial Biofilms: Methods and Protocols. G. Donelli. New York, NY, Springer New York: 3-22.

Stewart, P. S. and M. J. Franklin (2008). "Physiological heterogeneity in biofilms." Nature Reviews Microbiology 6(3): 199-210.

Storm, D. R., K. S. Rosenthal and P. E. Swanson (1977). "Polymyxin and Related Peptide Antibiotics." Annual Review of Biochemistry 46(1): 723-763.

Tan, J. H., C. Vidaillac, J. K. H. Yam, S. L. Chua, M. Givskov and L. Yang (2017). "Invitro and in-vivo efficacy of LpxC inhibitor, CHIR-090, alone or combined with colistin against Pseudomonas aeruginosa biofilm." Antimicrobial Agents and Chemotherapy 61: e02223-02216.

Tan, S. Y.-Y., S.-L. Chua, Y. Chen, S. A. Rice, S. Kjelleberg, T. E. Nielsen, L. Yang and M. Givskov (2013). "Identification of Five Structurally Unrelated Quorum-Sensing Inhibitors of Pseudomonas aeruginosa from a Natural-Derivative Database."

Antimicrobial Agents and Chemotherapy 57(11): 5629-5641.

The Gene Ontology, C. (2015). "Gene Ontology Consortium: going forward." Nucleic Acids Research 43(Database issue): D1049-D1056.

Thien-Fah Mah (2012). "Biofilm-specific antibiotic resistance." Future Microbiology 7(9): 1061-1072.

Tolker-Nielsen, T. and C. Sternberg (2011). Growing and Analyzing Biofilms in Flow Chambers. Current Protocols in Microbiology, John Wiley \& Sons, Inc.

Tuomanen, E., R. Cozens, W. Tosch, O. Zak and A. Tomasz (1986). "The rate of killing of Escherichia coli by beta-lactam antibiotics is strictly proportional to the rate of bacterial growth." Journal of General Microbiology 132(5): 1297-1304.

Ueda, A. and T. K. Wood (2009). "Connecting Quorum Sensing, c-di-GMP, Pel Polysaccharide, and Biofilm Formation in Pseudomonas aeruginosa through Tyrosine Phosphatase TpbA (PA3885)." PLOS Pathogens 5(6): e1000483.

Ueda, A. and T. K. Wood (2010). "Tyrosine Phosphatase TpbA of Pseudomonas aeruginosa Controls Extracellular DNA via Cyclic Diguanylic Acid Concentrations." Environmental Microbiology 2(3): 449-455.

Valentini, M. and A. Filloux (2016). "Biofilms and Cyclic di-GMP (c-di-GMP) Signaling: Lessons from Pseudomonas aeruginosa and Other Bacteria." Journal of Biological Chemistry 291(24): 12547-12555.

Valentini, M. and A. Filloux (2016). "Biofilms and Cyclic di-GMP (c-di-GMP) Signaling: Lessons from Pseudomonas aeruginosa and Other Bacteria." The Journal of Biological Chemistry 291(24): 12547-12555. 
Van den Driessche, F., P. Rigole, G. Brackman and T. Coenye (2014). "Optimization of resazurin-based viability staining for quantification of microbial biofilms." J Microbiol Methods 98: 31-34.

Vidaillac, C., L. Benichou and R. E. Duval (2012). "In-Vitro Synergy of Colistin Combinations against Colistin-Resistant Acinetobacter baumannii, Pseudomonas aeruginosa, and Klebsiella pneumoniae Isolates." Antimicrobial Agents and Chemotherapy 56(9): 4856-4861.

Vignaroli, C., C. Rinaldi and P. E. Varaldo (2011). "Striking "Seesaw Effect" between Daptomycin Nonsusceptibility and $\beta$-Lactam Susceptibility in Staphylococcus haemolyticus." Antimicrobial Agents and Chemotherapy 55(5): 2495-2497.

Wagner, S., R. Sommer, S. Hinsberger, C. Lu, R. W. Hartmann, M. Empting and A. Titz (2016). "Novel Strategies for the Treatment of Pseudomonas aeruginosa Infections." Journal of Medicinal Chemistry 59(13): 5929-5969.

Wei, Q. and L. Z. Ma (2013). "Biofilm Matrix and Its Regulation in Pseudomonas aeruginosa." International Journal of Molecular Sciences 14(10): 20983-21005.

Wei, Q., S. Tarighi, A. Dötsch, S. Häussler, M. Müsken, V. J. Wright, M. Cámara, P. Williams, S. Haenen, B. Boerjan, A. Bogaerts, E. Vierstraete, P. Verleyen, L. Schoofs, R. Willaert, V. N. De Groote, J. Michiels, K. Vercammen, A. Crabbé and P. Cornelis (2011). "Phenotypic and Genome-Wide Analysis of an Antibiotic-Resistant Small Colony Variant (SCV) of Pseudomonas aeruginosa." PLoS ONE 6(12): e29276.

Wessel, A. K., T. A. Arshad, M. Fitzpatrick, J. L. Connell, R. T. Bonnecaze, J. B. Shear and M. Whiteley (2014). "Oxygen Limitation within a Bacterial Aggregate." mBio 5(2): e00992-00914.

Westbrock-Wadman, S., D. R. Sherman, M. J. Hickey, S. N. Coulter, Y. Q. Zhu, P. Warrener, L. Y. Nguyen, R. M. Shawar, K. R. Folger and C. K. Stover (1999). "Characterization of a Pseudomonas aeruginosa Efflux Pump Contributing to Aminoglycoside Impermeability." Antimicrobial Agents and Chemotherapy 43(12): 2975-2983.

Whiteley, M., G. Bangera, R. Bumgarner, M. Parsek, G. Teitzel, S. Lory and E. P. Greenberg (2001). Gene expression in Pseudomonas aeruginosa biofilms.

Wiegand, I., K. Hilpert and R. E. W. Hancock (2008). "Agar and broth dilution methods to determine the minimal inhibitory concentration (MIC) of antimicrobial substances." Nature Protocols 3: 163.

Williamson, K. S., L. A. Richards, A. C. Perez-Osorio, B. Pitts, K. Mclnnerney, P. S. Stewart and M. J. Franklin (2012). "Heterogeneity in Pseudomonas aeruginosa Biofilms Includes Expression of Ribosome Hibernation Factors in the AntibioticTolerant Subpopulation and Hypoxia-Induced Stress Response in the Metabolically Active Population." Journal of Bacteriology 194(8): 2062-2073. 
Wolter, D. J. and P. D. Lister (2013). "Mechanisms of $\beta$-lactam resistance among Pseudomonas aeruginosa." Current pharmaceutical design 19(2): 209-222.

Workentine, M. L., C. D. Sibley, B. Glezerson, S. Purighalla, J. C. Norgaard-Gron, M. D. Parkins, H. R. Rabin and M. G. Surette (2013). "Phenotypic Heterogeneity of Pseudomonas aeruginosa Populations in a Cystic Fibrosis Patient." PLoS ONE 8(4): e60225.

World Health Organization. (2016). "United Nations high-level meeting on antimicrobial resistance." Retrieved September 2016, 2016, from http://www.who.int/antimicrobial-resistance/events/UNGA-meeting-amr-sept2016/en/.

World Health Organization (2017). "Global priority list of antibiotic-resistant bacteria to guide research, discovery, and development of new antibiotics." Geneva: World Health Organization.

Wright, E. A., J. L. Fothergill, S. Paterson, M. A. Brockhurst and C. Winstanley (2013). "Sub-inhibitory concentrations of some antibiotics can drive diversification of Pseudomonas aeruginosa populations in artificial sputum medium." BMC Microbiology 13(170).

Wu, H., C. Moser, H.-Z. Wang, N. Høiby and Z.-J. Song (2015). "Strategies for combating bacterial biofilm infections." International Journal of Oral Science 7(1): 1-7.

Yang, L., Y. Hu, Y. Liu, J. Zhang, J. Ulstrup and S. Molin (2011). "Distinct roles of extracellular polymeric substances in Pseudomonas aeruginosa biofilm development." Environmental Microbiology 13(7): 1705-1717.

Yin, W., H. Li, Y. Shen, Z. Liu, S. Wang, Z. Shen, R. Zhang, T. R. Walsh, J. Shen and Y. Wang (2017). "Novel Plasmid-Mediated Colistin Resistance Gene mcr-3 in Escherichia coli." $\underline{\mathrm{mBio}}$ 8(3).

Yu, S., T. Su, H. Wu, S. Liu, D. Wang, T. Zhao, Z. Jin, W. Du, M.-J. Zhu, S. L. Chua, L. Yang, D. Zhu, L. Gu and L. Z. Ma (2015). "PsIG, a self-produced glycosyl hydrolase, triggers biofilm disassembly by disrupting exopolysaccharide matrix." Cell Research 25(12): 1352-1367.

Zavascki, A. P., C. G. Carvalhaes, R. C. Picao and A. C. Gales (2010). "Multidrugresistant Pseudomonas aeruginosa and Acinetobacter baumannii: resistance mechanisms and implications for therapy." Expert Review of Anti-infective Therapy 8(1): 71-93.

Zhanel, G. G., R. Wiebe, L. Dilay, K. Thomson, E. Rubinstein, D. J. Hoban, A. M. Noreddin and J. A. Karlowsky (2007). "Comparative review of the carbapenems." Drugs 67(7): 1027-1052.

Zhou, A., T. M. Kang, J. Yuan, C. Beppler, C. Nguyen, Z. Mao, M. Q. Nguyen, P. Yeh and J. H. Miller (2015). "Synergistic Interactions of Vancomycin with Different Antibiotics against Escherichia coli: Trimethoprim and Nitrofurantoin Display Strong 
Synergies with Vancomycin against Wild-Type E. coli." Antimicrobial Agents and Chemotherapy 59(1): 276-281. 


\section{Appendix}

Supplementary Table 1 SNP between parent and colistin resistant $\mathrm{PAO} 1 / \mathrm{P}_{B A D^{-}} y$ hjH by pairwise comparison of whole genome sequencing.

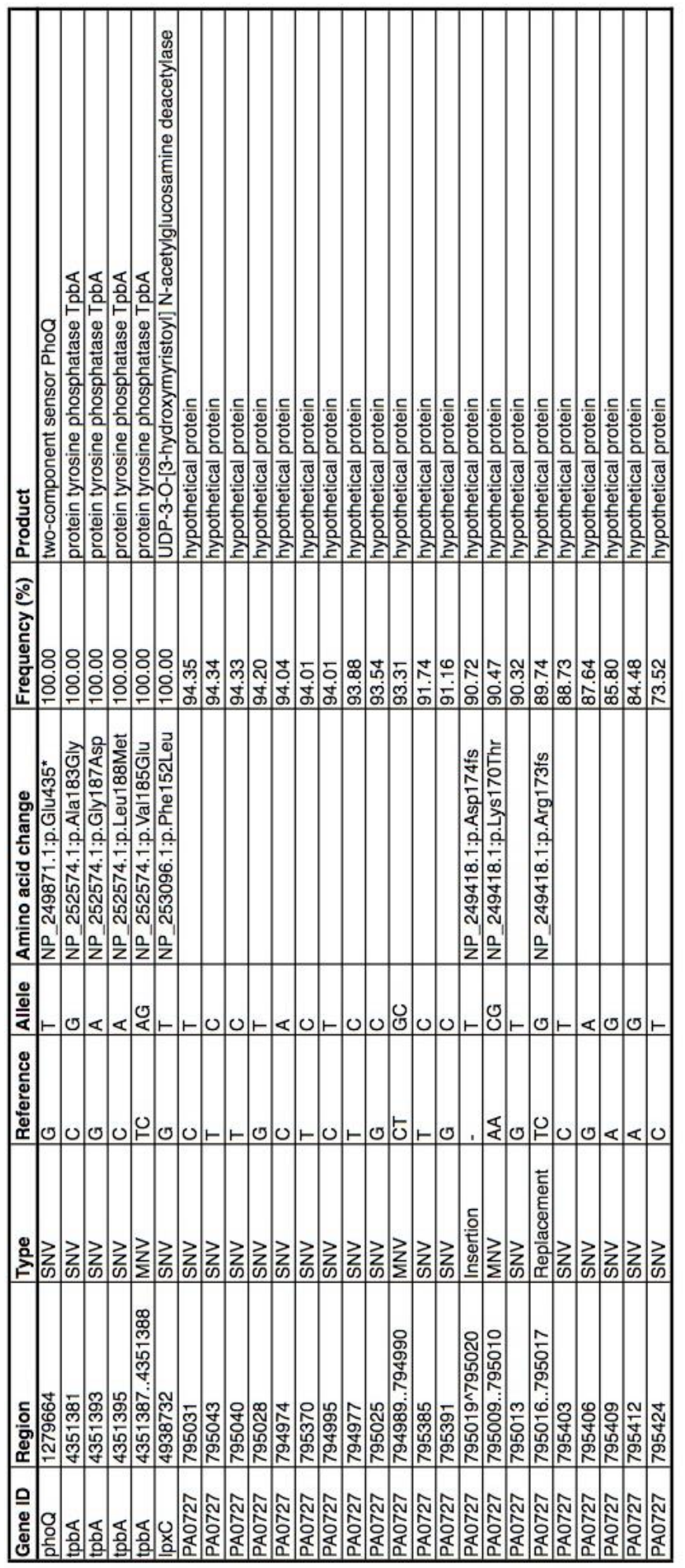


Supplementary Table 2 Differentialy upregulated genes in the colistin resistant



\begin{tabular}{|c|c|c|}
\hline Gene ID & log2FoldChange & PseudoCAP Function Class \\
\hline PA3559 & 8.271 & Putative enzymes \\
\hline $\operatorname{arn} A$ & 8.206 & Putative enzymes; Adaptation, Protection; Antibiotic resistance and susceptibility; Cell wall / LPS / capsule \\
\hline arnC & 8.173 & Adaptation, Protection; Putative enzymes; Cell wall / LPS / capsule; Antibiotic resistance and susceptibility \\
\hline arnB & 8.094 & Cell wall / LPS / capsule; Antibiotic resistance and susceptibility; Adaptation, Protection \\
\hline arnD & 7.735 & Adaptation, Protection; Antibiotic resistance and susceptibility; Cell wall / LPS / capsule \\
\hline $\operatorname{arnT}$ & 7.324 & Adaptation, Protection; Membrane proteins; Cell wall / LPS / capsule \\
\hline arnE & 6.747 & Adaptation, Protection; Cell wall / LPS / capsule; Membrane proteins \\
\hline arnF & 6.591 & Membrane proteins; Adaptation, Protection; Cell wall / LPS / capsule \\
\hline $\mathrm{oprH}$ & 6.242 & Membrane proteins; Adaptation, Protection; Transport of small molecules \\
\hline phoP & 5.665 & Transcriptional regulators; Two-component regulatory systems \\
\hline phoQ & 5.264 & Two-component regulatory systems \\
\hline PA4517 & 4.654 & Hypothetical, unclassified, unknown \\
\hline tpbA & 4.652 & Motility \& Attachment; Translation, post-translational modification, degradation \\
\hline PA4011 & 4.191 & Membrane proteins \\
\hline gcvP2 & 4.071 & Central intermediary metabolism; Amino acid biosynthesis and metabolism \\
\hline $\operatorname{dct} A$ & 3.944 & Transport of small molecules \\
\hline PA4010 & 3.893 & Hypothetical, unclassified, unknown \\
\hline gcvH2 & 3.467 & Amino acid biosynthesis and metabolism \\
\hline PA1181 & 2.870 & Membrane proteins \\
\hline inaA & 2.716 & Adaptation, Protection \\
\hline glyA2 & 2.613 & Amino acid biosynthesis and metabolism \\
\hline PA4379 & 2.606 & Hypothetical, unclassified, unknown \\
\hline PA5168 & 2.557 & Membrane proteins; Transport of small molecules \\
\hline dgkA & 2.515 & Fatty acid and phospholipid metabolism \\
\hline PA5167 & 2.448 & Membrane proteins; Transport of small molecules \\
\hline PA0921 & 2.397 & Membrane proteins \\
\hline PA5169 & 2.309 & Membrane proteins; Transport of small molecules \\
\hline PA1053 & 2.271 & Membrane proteins \\
\hline PA4635 & 2.239 & Hypothetical, unclassified, unknown \\
\hline PA2112 & 2.212 & Hypothetical, unclassified, unknown \\
\hline PA1972 & 2.105 & Membrane proteins \\
\hline PA1344 & 2.087 & Putative enzymes \\
\hline PA4455 & 2.001 & Membrane proteins; Transport of small molecules \\
\hline PA2110 & 1.992 & Hypothetical, unclassified, unknown \\
\hline PA2111 & 1.968 & Hypothetical, unclassified, unknown \\
\hline PA4824 & 1.966 & Hypothetical, unclassified, unknown \\
\hline PA0920 & 1.961 & Membrane proteins \\
\hline PA4586 & 1.921 & Membrane proteins \\
\hline opdO & 1.918 & Transport of small molecules; Membrane proteins \\
\hline PA2173 & 1.852 & Hypothetical, unclassified, unknown \\
\hline PA0924 & 1.841 & Hypothetical, unclassified, unknown; Membrane proteins \\
\hline liuA & 1.841 & Carbon compound catabolism \\
\hline $\mathrm{mgtA}$ & 1.822 & Transport of small molecules \\
\hline PA3310 & 1.807 & Membrane proteins \\
\hline PA4456 & 1.799 & Transport of small molecules \\
\hline PA4773 & 1.787 & Hypothetical, unclassified, unknown \\
\hline liuR & 1.739 & Transcriptional regulators \\
\hline $\mathrm{pcoA}$ & 1.732 & Adaptation, Protection \\
\hline PA0320 & 1.708 & Hypothetical, unclassified, unknown \\
\hline $\mathrm{pcoB}$ & 1.650 & Adaptation, Protection \\
\hline PA2159 & 1.627 & Hypothetical, unclassified, unknown \\
\hline PA1592 & 1.619 & Hypothetical, unclassified, unknown \\
\hline aruG & 1.613 & Amino acid biosynthesis and metabolism \\
\hline PA2116 & 1.597 & Hypothetical, unclassified, unknown \\
\hline PA5530 & 1.578 & Membrane proteins; Transport of small molecules \\
\hline PA4454 & 1.569 & Hypothetical, unclassified, unknown \\
\hline PA4452 & 1.560 & Hypothetical, unclassified, unknown \\
\hline PA2114 & 1.554 & Membrane proteins; Transport of small molecules \\
\hline psin & 1.541 & Hypothetical, unclassified, unknown \\
\hline $\mathrm{glpD}$ & 1.540 & Central intermediary metabolism; Energy metabolism \\
\hline PA4596 & 1.536 & Transcriptional regulators \\
\hline PA3274 & 1.515 & Hypothetical, unclassified, unknown \\
\hline PA0049 & 1.513 & Hypothetical, unclassified, unknown \\
\hline PA4453 & 1.503 & Hypothetical, unclassified, unknown \\
\hline sdaA & 1.502 & Amino acid biosynthesis and metabolism \\
\hline PA4822 & 1.484 & Membrane proteins \\
\hline PA2109 & 1.474 & Hypothetical, unclassified, unknown \\
\hline PA2457 & 1.472 & Hypothetical, unclassified, unknown \\
\hline PA1323 & 1.467 & Hypothetical, unclassified, unknown \\
\hline PA4382 & 1.440 & Hypothetical, unclassified, unknown \\
\hline PA1688 & 1.439 & Hypothetical, unclassified, unknown \\
\hline liuB & 1.437 & Carbon compound catabolism \\
\hline PA3523 & 1.431 & Transport of small molecules \\
\hline
\end{tabular}




\begin{tabular}{|c|c|c|}
\hline PA4774 & 1.428 & Hypothetical, unclassified, unknown \\
\hline glpK & 1.428 & Central intermediary metabolism \\
\hline PA2557 & 1.409 & Fatty acid and phospholipid metabolism \\
\hline PA1471 & 1.407 & Hypothetical, unclassified, unknown \\
\hline rfaD & 1.400 & Cell wall / LPS / capsule \\
\hline PA4377 & 1.398 & Hypothetical, unclassified, unknown \\
\hline aruF & 1.391 & Amino acid biosynthesis and metabolism \\
\hline PA3691 & 1.391 & Hypothetical, unclassified, unknown \\
\hline PA5526 & 1.382 & Hypothetical, unclassified, unknown \\
\hline PA2167 & 1.377 & Hypothetical, unclassified, unknown \\
\hline PA1404 & 1.356 & Hypothetical, unclassified, unknown \\
\hline pfpl & 1.356 & Translation, post-translational modification, degradation \\
\hline plcR & 1.355 & Secreted Factors (toxins, enzymes, alginate) \\
\hline PA5212 & 1.351 & Hypothetical, unclassified, unknown \\
\hline opmD & 1.350 & Membrane proteins; Transport of small molecules \\
\hline PA4826 & 1.346 & Hypothetical, unclassified, unknown \\
\hline PA1345 & 1.338 & Hypothetical, unclassified, unknown \\
\hline PA0833 & 1.335 & Membrane proteins \\
\hline liuC & 1.324 & Carbon compound catabolism \\
\hline PA0919 & 1.320 & Hypothetical, unclassified, unknown \\
\hline PA3819 & 1.318 & Membrane proteins \\
\hline osmE & 1.315 & Membrane proteins; Adaptation, Protection \\
\hline PA2486 & 1.311 & Hypothetical, unclassified, unknown \\
\hline PA4023 & 1.310 & Transport of small molecules \\
\hline polA & 1.309 & DNA replication, recombination, modification and repair \\
\hline PA2171 & 1.288 & Hypothetical, unclassified, unknown \\
\hline PA4877 & 1.283 & Hypothetical, unclassified, unknown \\
\hline PA0737 & 1.277 & Hypothetical, unclassified, unknown \\
\hline PA1689 & 1.271 & Hypothetical, unclassified, unknown \\
\hline PA5183 & 1.261 & Membrane proteins \\
\hline PA4172 & 1.261 & DNA replication, recombination, modification and repair \\
\hline PA2164 & 1.255 & Putative enzymes \\
\hline PA2168 & 1.254 & Hypothetical, unclassified, unknown \\
\hline fecA & 1.254 & Membrane proteins; Transport of small molecules \\
\hline PA4577 & 1.249 & Hypothetical, unclassified, unknown \\
\hline gcvT2 & 1.232 & Central intermediary metabolism; Amino acid biosynthesis and metabolism \\
\hline PA1196 & 1.225 & Transcriptional regulators \\
\hline PA5460 & 1.225 & Hypothetical, unclassified, unknown \\
\hline PA2143 & 1.216 & Hypothetical, unclassified, unknown \\
\hline PA2807 & 1.209 & Hypothetical, unclassified, unknown \\
\hline liuD & 1.202 & Carbon compound catabolism \\
\hline osmC & 1.199 & Adaptation, Protection \\
\hline mexl & 1.193 & Membrane proteins; Transport of small molecules \\
\hline treA & 1.192 & Carbon compound catabolism \\
\hline PA4828 & 1.189 & Hypothetical, unclassified, unknown \\
\hline PA0490 & 1.184 & Hypothetical, unclassified, unknown \\
\hline PA1324 & 1.178 & Hypothetical, unclassified, unknown \\
\hline PA1494 & 1.164 & Hypothetical, unclassified, unknown \\
\hline ampDh3 & 1.154 & Antibiotic resistance and susceptibility \\
\hline PA2562 & 1.151 & Hypothetical, unclassified, unknown \\
\hline aruD & 1.150 & Amino acid biosynthesis and metabolism \\
\hline PA2190 & 1.147 & Hypothetical, unclassified, unknown \\
\hline PA3519 & 1.146 & Hypothetical, unclassified, unknown \\
\hline PA2163 & 1.138 & Hypothetical, unclassified, unknown \\
\hline PA0462 & 1.136 & Hypothetical, unclassified, unknown \\
\hline PA4357 & 1.136 & Hypothetical, unclassified, unknown \\
\hline PA2046 & 1.134 & Hypothetical, unclassified, unknown \\
\hline $\operatorname{arcD}$ & 1.132 & Membrane proteins; Amino acid biosynthesis and metabolism; Transport of small molecules \\
\hline PA4359 & 1.131 & Hypothetical, unclassified, unknown \\
\hline PA3839 & 1.128 & Membrane proteins; Transport of small molecules \\
\hline psrA & 1.122 & Transcriptional regulators \\
\hline PA2414 & 1.121 & Carbon compound catabolism \\
\hline PA2415 & 1.120 & Membrane proteins \\
\hline PA2169 & 1.118 & Hypothetical, unclassified, unknown \\
\hline PA2021 & 1.104 & Hypothetical, unclassified, unknown \\
\hline PA2655 & 1.098 & Hypothetical, unclassified, unknown \\
\hline PA4610 & 1.096 & Hypothetical, unclassified, unknown \\
\hline mucA & 1.095 & Transcriptional regulators; Cell wall / LPS / capsule \\
\hline IptF & 1.094 & Membrane proteins; Transport of small molecules \\
\hline PA0028 & 1.093 & Hypothetical, unclassified, unknown \\
\hline PA5027 & 1.082 & Hypothetical, unclassified, unknown \\
\hline PA1111 & 1.072 & Hypothetical, unclassified, unknown \\
\hline mexH & 1.069 & Transport of small molecules \\
\hline PA3459 & 1.068 & Amino acid biosynthesis and metabolism \\
\hline
\end{tabular}




\begin{tabular}{|l|l|l|}
\hline glcF & 1.066 & Central intermediary metabolism; Carbon compound catabolism \\
\hline PA3278 & 1.058 & Membrane proteins \\
\hline PA2160 & 1.056 & Putative enzymes \\
\hline PA0062 & 1.051 & Hypothetical, unclassified, unknown \\
\hline phzH & 1.045 & Putative enzymes \\
\hline PA4878 & 1.043 & Transcriptional regulators \\
\hline PA3922 & 1.037 & Hypothetical, unclassified, unknown \\
\hline PA3460 & 1.035 & Putative enzymes \\
\hline PA2134 & 1.028 & Hypothetical, unclassified, unknown \\
\hline PA2753 & 1.026 & Hypothetical, unclassified, unknown \\
\hline PA1282 & 1.024 & Membrane proteins; Transport of small molecules \\
\hline PA4358 & 1.019 & Membrane proteins; Transport of small molecules \\
\hline glpF & 1.018 & Transport of small molecules \\
\hline PA4338 & 1.014 & Membrane proteins \\
\hline PA5182 & 1.013 & Membrane proteins \\
\hline PA3880 & 1.010 & Hypothetical, unclassified, unknown \\
\hline phuR & 1.007 & Transport of small molecules \\
\hline PA0460 & 1.004 & Hypothetical, unclassified, unknown \\
\hline
\end{tabular}


Supplementary Table 3 Differentialy downregulated genes in the colistin resistant

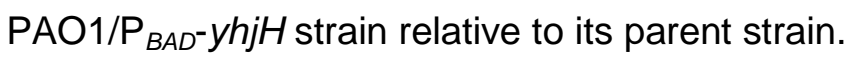

\begin{tabular}{|c|c|c|}
\hline Gene ID & log2foldChange & PseudoCAP Function Class \\
\hline PA3441 & -3.637 & Transport of small molecules \\
\hline PA3443 & -2.900 & Membrane proteins; Transport of small molecules \\
\hline PA3442 & -2.758 & Transport of small molecules \\
\hline PA2309 & -2.365 & Hypothetical, unclassified, unknown \\
\hline PA5181 & -2.304 & Putative enzymes \\
\hline tauD & -2.300 & Carbon compound catabolism \\
\hline PA3445 & -2.253 & Hypothetical, unclassified, unknown \\
\hline PA2840 & -2.212 & Transcription, RNA processing and degradation \\
\hline PA2086 & -2.178 & Carbon compound catabolism \\
\hline PA5099 & -2.171 & Membrane proteins; Transport of small molecules \\
\hline hutU & -2.151 & Amino acid biosynthesis and metabolism \\
\hline hutH & -2.140 & Amino acid biosynthesis and metabolism \\
\hline PA2310 & -2.044 & Putative enzymes \\
\hline PA2308 & -2.017 & Transport of small molecules \\
\hline PA2307 & -2.014 & Membrane proteins; Transport of small molecules \\
\hline $\mathrm{rpmB}$ & -1.977 & Translation, post-translational modification, degradation \\
\hline PA5470 & -1.934 & Translation, post-translational modification, degradation \\
\hline PA3931 & -1.932 & Hypothetical, unclassified, unknown \\
\hline PA5097 & -1.907 & Membrane proteins; Transport of small molecules \\
\hline PA3447 & -1.846 & Transport of small molecules \\
\hline PA3446 & -1.782 & Hypothetical, unclassified, unknown \\
\hline PA2085 & -1.765 & Carbon compound catabolism \\
\hline PA3741 & -1.758 & Hypothetical, unclassified, unknown \\
\hline PA3932 & -1.736 & Transcriptional regulators \\
\hline rpmG & -1.734 & Translation, post-translational modification, degradation \\
\hline PA3938 & -1.706 & Transport of small molecules \\
\hline PA5471 & -1.660 & Antibiotic resistance and susceptibility \\
\hline PA3936 & -1.623 & Membrane proteins; Transport of small molecules \\
\hline PA3450 & -1.611 & Adaptation, Protection \\
\hline PA2050 & -1.597 & Transcriptional regulators \\
\hline PA4191 & -1.566 & Putative enzymes \\
\hline mdcD & -1.556 & Carbon compound catabolism \\
\hline PA0578 & -1.552 & Hypothetical, unclassified, unknown \\
\hline PA2090 & -1.530 & Hypothetical, unclassified, unknown \\
\hline PA5106 & -1.499 & Hypothetical, unclassified, unknown \\
\hline rpsT & -1.486 & Central intermediary metabolism; Translation, post-translational modification, degradation \\
\hline atsA & -1.473 & Central intermediary metabolism; Carbon compound catabolism \\
\hline rplS & -1.464 & Translation, post-translational modification, degradation \\
\hline PA4918 & -1.459 & Hypothetical, unclassified, unknown \\
\hline PA4195 & -1.457 & Transport of small molecules \\
\hline PA4881 & -1.451 & Hypothetical, unclassified, unknown \\
\hline PA4933 & -1.441 & Membrane proteins \\
\hline PA2312 & -1.435 & Transcriptional regulators \\
\hline PA5072 & -1.429 & Adaptation, Protection; Chemotaxis \\
\hline PA5096 & -1.414 & Transport of small molecules \\
\hline PA4582 & -1.406 & Hypothetical, unclassified, unknown \\
\hline PA5383 & -1.392 & Hypothetical, unclassified, unknown \\
\hline $\operatorname{trmD}$ & -1.383 & Transcription, RNA processing and degradation \\
\hline PA5180 & -1.377 & Hypothetical, unclassified, unknown \\
\hline PA4355 & -1.362 & Membrane proteins; Transport of small molecules; Transcriptional regulators \\
\hline pscB & -1.354 & Protein secretion/export apparatus \\
\hline ibpA & -1.342 & Chaperones \& heat shock proteins \\
\hline PA3937 & -1.329 & Transport of small molecules \\
\hline PA5103 & -1.324 & Hypothetical, unclassified, unknown \\
\hline PA2597 & -1.309 & Putative enzymes \\
\hline PA5504 & -1.287 & Membrane proteins; Transport of small molecules \\
\hline PA0191 & -1.286 & Transcriptional regulators \\
\hline PA2328 & -1.286 & Hypothetical, unclassified, unknown \\
\hline PA3444 & -1.283 & Putative enzymes \\
\hline PA2600 & -1.274 & Hypothetical, unclassified, unknown \\
\hline PA2019 & -1.259 & Transport of small molecules; Antibiotic resistance and susceptibility \\
\hline PA2326 & -1.252 & Putative enzymes \\
\hline PA0805 & -1.246 & Hypothetical, unclassified, unknown \\
\hline PA2083 & -1.246 & Carbon compound catabolism \\
\hline hutG & -1.235 & Amino acid biosynthesis and metabolism \\
\hline PA2594 & -1.230 & Hypothetical, unclassified, unknown \\
\hline hslU & -1.216 & Chaperones \& heat shock proteins \\
\hline PA2204 & -1.215 & Transport of small molecules \\
\hline PA2334 & -1.210 & Transcriptional regulators \\
\hline $\operatorname{grpE}$ & -1.210 & DNA replication, recombination, modification and repair; Chaperones \& heat shock proteins \\
\hline PA5095 & -1.203 & Membrane proteins; Transport of small molecules \\
\hline rpsR & -1.201 & Translation, post-translational modification, degradation \\
\hline hutl & -1.199 & Amino acid biosynthesis and metabolism \\
\hline
\end{tabular}




\begin{tabular}{|c|c|c|}
\hline rplJ & -1.195 & Translation, post-translational modification, degradation \\
\hline PA5503 & -1.189 & Transport of small molecules \\
\hline PA2018 & -1.176 & Transport of small molecules; Membrane proteins; Antibiotic resistance and susceptibility \\
\hline PA2540 & -1.175 & Hypothetical, unclassified, unknown \\
\hline PA4220 & -1.172 & Hypothetical, unclassified, unknown \\
\hline pscD & -1.166 & Protein secretion/export apparatus \\
\hline rpll & -1.163 & Translation, post-translational modification, degradation \\
\hline PA2971 & -1.162 & Hypothetical, unclassified, unknown \\
\hline gltB & -1.161 & Amino acid biosynthesis and metabolism \\
\hline PA2359 & -1.157 & Transcriptional regulators \\
\hline mreD & -1.157 & Cell wall / LPS / capsule; Cell division \\
\hline PA1501 & -1.156 & Hypothetical, unclassified, unknown \\
\hline pncB1 & -1.156 & Biosynthesis of cofactors, prosthetic groups and carriers \\
\hline PA3600 & -1.153 & Translation, post-translational modification, degradation \\
\hline $\mathrm{rps} F$ & -1.137 & Translation, post-translational modification, degradation \\
\hline PA0434 & -1.134 & Membrane proteins \\
\hline PA5094 & -1.129 & Transport of small molecules \\
\hline PA0277 & -1.128 & Hypothetical, unclassified, unknown \\
\hline PA2598 & -1.124 & Hypothetical, unclassified, unknown \\
\hline $\operatorname{arsB}$ & -1.118 & Membrane proteins; Transport of small molecules \\
\hline PA1791 & -1.116 & Hypothetical, unclassified, unknown \\
\hline dnaK & -1.111 & DNA replication, recombination, modification and repair; Adaptation, Protection; Chaperones \& heat shock proteins \\
\hline PA2084 & -1.111 & Amino acid biosynthesis and metabolism \\
\hline flhA & -1.101 & Chemotaxis; Adaptation, Protection; Motility \& Attachment \\
\hline mdcE & -1.096 & Carbon compound catabolism \\
\hline aprE & -1.094 & Secreted Factors (toxins, enzymes, alginate); Protein secretion/export apparatus \\
\hline PA2786 & -1.092 & Hypothetical, unclassified, unknown \\
\hline rplu & -1.089 & Translation, post-translational modification, degradation \\
\hline PA0879 & -1.088 & Putative enzymes \\
\hline thil & -1.087 & Biosynthesis of cofactors, prosthetic groups and carriers \\
\hline clpB & -1.086 & Translation, post-translational modification, degradation \\
\hline PA5090 & -1.085 & Hypothetical, unclassified, unknown \\
\hline rbfa & -1.084 & Translation, post-translational modification, degradation; Adaptation, Protection \\
\hline hutC & -1.069 & Transcriptional regulators \\
\hline PA1697 & -1.062 & Protein secretion/export apparatus \\
\hline PA3939 & -1.059 & Hypothetical, unclassified, unknown \\
\hline rplL & -1.056 & Translation, post-translational modification, degradation \\
\hline arsR & -1.051 & Transcriptional regulators \\
\hline aprF & -1.026 & Secreted Factors (toxins, enzymes, alginate); Protein secretion/export apparatus \\
\hline $\mathrm{rpmA}$ & -1.019 & Translation, post-translational modification, degradation \\
\hline PA2327 & -1.019 & Membrane proteins; Transport of small molecules \\
\hline pscC & -1.005 & Protein secretion/export apparatus \\
\hline
\end{tabular}

